\title{
Synthesis of Sulfinamidines and Sulfinimidate Esters by Transfer of Nitrogen to Sulfenamides
}

\author{
Michael Andresini, ${ }^{\mathrm{a}, \star}$ Mauro Spennacchio, ${ }^{\mathrm{a}, \grave{ }}$ Giuseppe Romanazzi, ${ }^{\mathrm{b}}$ Fulvio Ciriaco,${ }^{\mathrm{c}}$ Guy Clarkson, ${ }^{\mathrm{d}}$ \\ Leonardo Degennaro, ${ }^{\mathrm{a}, *}$ and Renzo Luisi, ${ }^{\mathrm{a}, *}$ \\ ${ }^{a}$ Flow Chemistry and Microreactor Technology FLAME-Lab, Department of Pharmacy - Drug Sciences, University of Bari \\ “A. Moro” Via E. Orabona 4, Bari, I - 70125. ${ }^{b}$ DICATECh, Politecnico di Bari, Via E. Orabona 4, Bari 70125, Italy. ${ }^{C}$ Di-

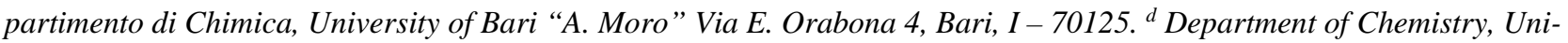 \\ versity of Warwick, Gibbet Hill Road, Coventry CV4 7AL, UK.
}

\section{SUPPORTING INFORMATION}

Table of Contents

1. General

2. Sulfenamides

2.1 General procedure A for sulfenamide synthesis S2

2.2 List of sulfenamides 1a-k S3

2.2 Characterization data for sulfenamides 1a-j $\quad S 3$

3. Synthesis of $\mathbf{N}$-mesyloxycarbamates

3.1 Synthesis of benzyl((methylsulfonyl)oxy)carbamate 2a $\quad$ S5

3.2 Synthesis of isobutyl((methylsulfonyl)oxy)carbamate 2b $\quad \mathbf{5 6}$

4. Sulfinimidate esters

4.1 General procedure B for sulfinimidate esters synthesis $\quad$ S7

4.2 Procedure $C$ for sulfinimidate ester 3a synthesis on 2 mmol scale $\quad 57$

4.3 Characterization data for sulfinimidate esters 3a-s $\quad$ S8

5. DoE optimization study for 4a synthesis $\quad \mathrm{S14}$

6. Sulfinamidines

6.1 General procedure D for sulfinamidines synthesis $\quad \mathrm{S16}$

6.2 Procedure E for sulfinamidine 4a synthesis on $2 \mathrm{mmol}$ scale $\quad \mathrm{S16}$

$\begin{array}{ll}\text { 6.3 Characterization data for sulfinamidines 4a-o } & \mathrm{S17}\end{array}$

7. Mechanistic investigation

7.1 Control experiments

7.2 ${ }^{1} \mathrm{H}$ NMR experiments: formation of $3 a \quad S 23$

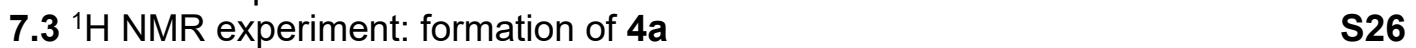

$\begin{array}{lr}\text { 7.4 Ab initio Computational Calculations } & \mathbf{S 2 7}\end{array}$

Copies of ${ }^{1} \mathrm{H}$ and ${ }^{13} \mathrm{C}$ NMR spectra $\quad$ S39

$\begin{array}{ll}\text { X-Ray analysis for compounds } 3 \mathrm{j} \text { and } 4 a & \mathbf{S}\end{array}$

$\begin{array}{lr}\text { References } & \mathbf{S 1 0 0}\end{array}$ 


\section{General}

Flash chromatography was performed using 230-400 mesh silica with the indicated solvent system according to standard techniques. Thin layer chromatography (TLC) was carried out on aluminium sheets precoated with silica gel 60F254 (Merck). The visualization of the spots was performed under UV light $(\lambda$ $=254 \mathrm{~nm})$ and/or $\mathrm{KMnO}_{4(\text { aq. })}$. Infrared spectra $\left(\mathrm{V}_{\max }, \mathrm{FT}-\mathrm{IR}\right)$ were recorded in reciprocal centimeters $(\mathrm{cm}$ 1). Nuclear magnetic resonance spectra were recorded with an Agilent 500 spectrometer $\left(500 \mathrm{MHz}\right.$ for ${ }^{1}$ $\mathrm{H}, 125 \mathrm{MHz}$ for ${ }^{13} \mathrm{C}, 470 \mathrm{MHz}$ for ${ }^{19} \mathrm{~F}$ ), and a Varian Mercury 300 spectrometer ( $300 \mathrm{MHz}$ for ${ }^{1} \mathrm{H}, 75 \mathrm{MHz}$ for ${ }^{13} \mathrm{C}, 282 \mathrm{MHz}$ for ${ }^{19} \mathrm{~F}$ ). The peak of the (residual) solvent signal was used as an internal standard which was related to TMS with $\delta 7.26 \mathrm{ppm}\left({ }^{1} \mathrm{H}\right.$ in $\left.\mathrm{CDCl}_{3}\right), \delta 77.00 \mathrm{ppm}\left({ }^{13} \mathrm{C}\right.$ in $\left.\mathrm{CDCl}_{3}\right)$. Absolute referencing was used for the ${ }^{19} \mathrm{~F}$ NMR spectra. Spin-spin coupling constants $(J)$ are given in Hz. HRMS spectra were recorded on Agilent 6530 accurate mass Q-TOF instrument using electrospray ion source (ESI) operated in positive ion mode. All the chemicals were purchased from Sigma-Aldrich, Alfa Aesar, Fluorochem and TCl Europe. Solutions were concentrated under reduced pressure with a rotary evaporator. 


\section{Sulfenamides}

\subsection{General procedure A for sulfenamides synthesis}

Prepared using the procedure reported from Bull, Luisi et al.. ${ }^{1}$

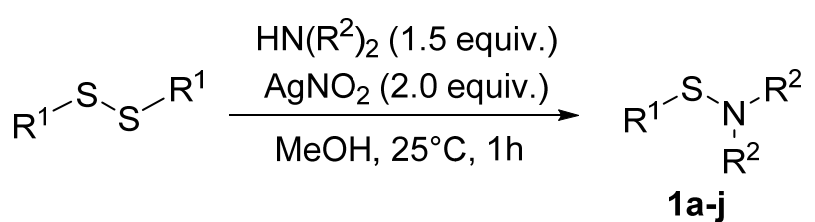

To a stirred solution of disulphide (1.0 equiv) in $\mathrm{MeOH}(0.1 \mathrm{M})$, amine (1.5 equiv) and silver nitrate (2.0 equiv) were added at $25^{\circ} \mathrm{C}$ for $1 \mathrm{~h}$. The reaction mixture was filtered and washed with $2 \times 50 \mathrm{~mL}$ of $\mathrm{MeOH}$. The solvent was removed under reduced pressure. $\mathrm{H}_{2} \mathrm{O}(75 \mathrm{~mL})$ was added to the crude mixture and the aqueous mixture extracted with $\mathrm{Et}_{2} \mathrm{O}(3 \times 25 \mathrm{~mL})$. The combined organic layers were dried $\left(\mathrm{Na}_{2} \mathrm{SO}_{4}\right)$, filtered and the solvent removed under reduced pressure. Purification by filtration through a basic alumina (activity IV) plug $(2 \mathrm{~cm}$ thick) afforded the corresponding pure sulfenamide.

\subsection{List of Sulfenamides 1a-k}

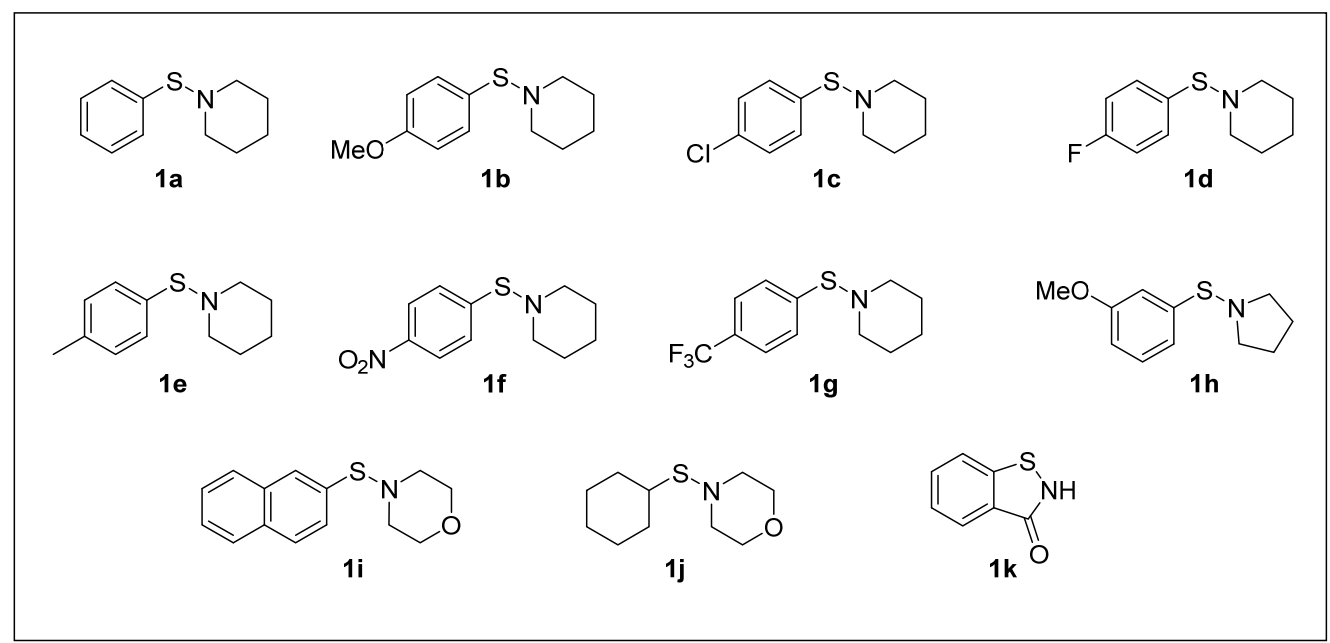

Sulfenamides 1a-j were synthetized according to General procedure A. Benzo[d]isothiazol-3(2H)-one $1 \mathrm{k}$ was purchased from Sigma-Aldrich and used without further purification. 


\subsection{Characterization data for sulfenamides $1 a-j$}

\section{1-(Phenylthio)piperidine (1a)}

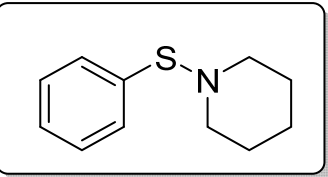

Prepared according to General Procedure A using 1,2-diphenyldisulfane (1,0 g, $4,587 \mathrm{~mol}, 1.0$ equiv). Purification by filtration through a plug of basic alumina (activity IV, $2 \mathrm{~cm}$ thick) with AcOEt $(300 \mathrm{~mL})$ afforded sulfenamide 1a $(736 \mathrm{mg}$, $83 \%)$ as a colourless oil. ${ }^{1} \mathrm{H}$ NMR $\left(500 \mathrm{MHz}, \mathrm{CDCl}_{3}, \mathrm{ppm}\right) \delta 7.44-7.42(\mathrm{~m}, 2 \mathrm{H}$, $\mathrm{Ar}-\mathrm{H}), 7.35-7.26(\mathrm{~m}, 2 \mathrm{H}, \mathrm{Ar}-\mathrm{H}), 7.25-7.23(\mathrm{~m}, 1 \mathrm{H}, \mathrm{Ar}-\mathrm{H}), 2.96-2.94(\mathrm{~m}, 4 \mathrm{H}$, $\left.\mathrm{CH}_{2} \mathrm{~N}\right), 1.66-1.62\left(\mathrm{~m}, 4 \mathrm{H}, \mathrm{CH}_{2} \mathrm{CH}_{2} \mathrm{~N}\right), 1.39-1.34\left(\mathrm{~m}, 2 \mathrm{H}, \mathrm{N}\left(\mathrm{CH}_{2}\right)_{2} \mathrm{CH}_{2}\right)$. Analytical data (NMR) in agreement with the literature. ${ }^{2}$

\section{1-[(4-Methoxyphenyl)thio]piperidine (1b)}

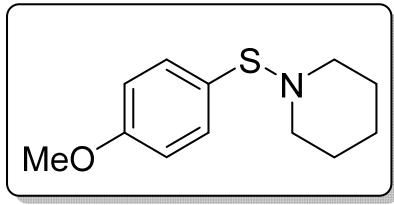

Prepared according to General Procedure A using 1,2-bis(4-methoxyphenyl)disulfane $(940 \mathrm{mg}, 3.38 \mathrm{mmol}, 1.0$ equiv). Purification by filtration through a plug of basic alumina (activity IV, $2 \mathrm{~cm}$ thick) using AcOEt $(300 \mathrm{~mL})$ afforded sulfenamide $1 \mathrm{~b}(664 \mathrm{mg}, 88 \%)$ as a yellow oil. ${ }^{1} \mathrm{H}$ NMR $(500 \mathrm{MHz}$, $\left.\mathrm{CDCl}_{3}, \mathrm{ppm}\right) \delta 7.48(\mathrm{~d}, J=8.8 \mathrm{~Hz}, 2 \mathrm{H}, 2 \times \mathrm{Ar}-\mathrm{H}), 6.89(\mathrm{~d}, J=8.8 \mathrm{~Hz}, 2 \mathrm{H}$, $\mathrm{Ar}-\mathrm{H}), 3.83\left(\mathrm{~s}, 3 \mathrm{H}, \mathrm{OCH}_{3}\right) 2.81\left(\mathrm{~m}, 4 \mathrm{H}, 2 \times \mathrm{NCH}_{2}\right), 1.64-1.56(\mathrm{~m}, 4 \mathrm{H}, 2 \times$ $\left.\mathrm{NCH}_{2} \mathrm{CH}_{2}\right), 1.29-1.22\left(\mathrm{~m}, 2 \mathrm{H}, \mathrm{N}(\mathrm{CH} 2)_{2} \mathrm{CH}_{2}\right)$. Analytical data (NMR) in agreement with the literature. ${ }^{3}$

\section{1-[(4-Chlorophenyl)thio]piperidine (1c)}

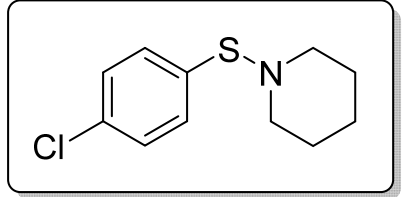

Prepared according to General Procedure A using 1,2-bis(4-chlorophenyl)disulfane (443 mg, $1.55 \mathrm{mmol}, 1.0$ equiv). Purification by filtration through a plug of basic alumina (activity IV, $2 \mathrm{~cm}$ thick) using AcOEt $(300 \mathrm{~mL})$ afforded sulfenamide $1 \mathrm{c}(282 \mathrm{mg}, 80 \%)$ as a colourless oil. ${ }^{1} \mathrm{H}$ NMR $\left(300 \mathrm{MHz}, \mathrm{CDCl}_{3}\right.$, ppm) $\delta 7.37-7.33(\mathrm{~m}, 2 \mathrm{H}, 2 \times \mathrm{Ar}-\mathrm{H}), 7.33-7.28(\mathrm{~m}, 2 \mathrm{H}, 2 \times \mathrm{Ar}-\mathrm{H}), 2.94-2.91$ $(\mathrm{m}, 4 \mathrm{H}, 2 \times \mathrm{NCH} 2), 1.67-1.60(\mathrm{~m}, 4 \mathrm{H}, 2 \times \mathrm{NCH} 2 \mathrm{CH} 2), 1.41-1.33\left(\mathrm{~m}, 2 \mathrm{H}, \mathrm{N}\left(\mathrm{CH}_{2}\right)_{2} \mathrm{CH}_{2}\right)$. Analytical data $(\mathrm{NMR})$ in agreement with the literature. ${ }^{3}$

\section{1-[(4-Fluorophenyl)thio]piperidine (1d)}

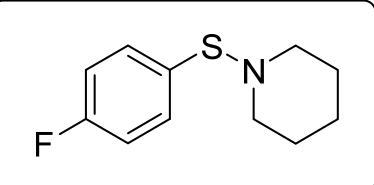

Prepared according to General Procedure A using 1,2-bis(4-fluorophenyl)disulfane ( $400 \mathrm{mg}, 1.58 \mathrm{mmol}, 1.0$ equiv). Purification by filtration through a plug of basic alumina (activity IV, $2 \mathrm{~cm}$ thick) using AcOEt $(300 \mathrm{~mL})$ afforded sulfenamide 1d (264 mg, 79\%) as a yellow oil. ${ }^{1} \mathrm{H}$ NMR $\left(300 \mathrm{MHz}, \mathrm{CDCl}_{3}\right) \delta$ 7.48-7.44 (m, 2H, $2 \times \mathrm{Ar}-\mathrm{H}), 7.08-7.02(\mathrm{~m}, 2 \mathrm{H}, 2 \times \mathrm{Ar}-\mathrm{H}), 2.89-2.85(\mathrm{~m}, 4 \mathrm{H}$, $\left.2 \times \mathrm{NCH}_{2}\right), 1.66-1.58\left(\mathrm{~m}, 4 \mathrm{H}, 2 \times \mathrm{NCH}_{2} \mathrm{CH}_{2}\right), 1.35-1.29\left(\mathrm{~m}, 2 \mathrm{H}, \mathrm{N}\left(\mathrm{CH}_{2}\right)_{2} \mathrm{CH}_{2}\right)$. Analytical data (NMR) in agreement with the literature. ${ }^{1}$

\section{1-[(4-Methylphenyl)thio]piperidine (1e)}

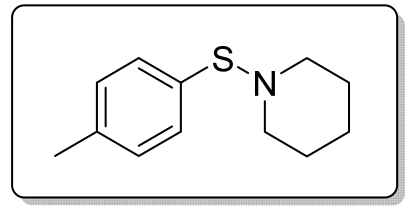

Prepared according to General Procedure A using 1,2-bis(4methylphenyl)disulfane ( $485 \mathrm{mg}, 1.97 \mathrm{mmol}, 1.0$ equiv). Purification by filtration through a plug of basic alumina (activity IV, $2 \mathrm{~cm}$ thick) using AcOEt (300 $\mathrm{mL}$ ) afforded sulfenamide $1 \mathrm{e}(344 \mathrm{mg}, 84 \%)$ as a colourless oil. ${ }^{1} \mathrm{H}$ NMR $(300$ $\left.\mathrm{MHz}, \mathrm{CDCl}_{3}\right) \delta 7.38(\mathrm{~d}, J=7.9 \mathrm{~Hz}, 2 \mathrm{H}), 7.16_{5}(\mathrm{~d}, J=7.9 \mathrm{~Hz}, 2 \mathrm{H}), 2.90-2.86$ $\left(\mathrm{m}, 4 \mathrm{H}, 2 \times \mathrm{NCH}_{2}\right), 2.36\left(\mathrm{~s}, 3 \mathrm{H}, \mathrm{Ar}-\mathrm{CH}_{3}\right), 1.65-1.58\left(\mathrm{~m}, 4 \mathrm{H}, 2 \times \mathrm{NCH}_{2} \mathrm{CH}_{2}\right), 1.34-1.29(\mathrm{~m}, 2 \mathrm{H}$, $\left.\mathrm{N}\left(\mathrm{CH}_{2}\right)_{2} \mathrm{CH}_{2}\right)$. Analytical data (NMR) in agreement with the in literature. ${ }^{3}$ 


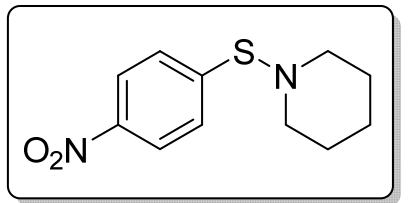

Prepared according to General Procedure A using 1,2-bis(4-nitrophenyl)disulfane (607 mg, $1.97 \mathrm{mmol}, 1.0$ equiv). Purification by filtration through a plug of basic alumina (activity IV, $2 \mathrm{~cm}$ thick) using AcOEt $(300 \mathrm{~mL})$ afforded sulfenamide $1 f(268 \mathrm{mg}, 57 \%)$ as a yellow solid. ${ }^{1} \mathrm{H} \mathrm{NMR}\left(500 \mathrm{MHz}, \mathrm{CDCl}_{3}, \mathrm{ppm}\right)$ ठ 8.15-8.13 (m, 2H, $2 \times \mathrm{Ar}-\mathrm{H}), 7.40-7.37(\mathrm{~m}, 2 \mathrm{H}, 2 \times \mathrm{Ar}-\mathrm{H}), 3.07-3.05(\mathrm{~m}$, $\left.4 \mathrm{H}, 2 \times \mathrm{NCH}_{2}\right), 1.70\left(\mathrm{q}, J=5.7 \mathrm{~Hz}, 4 \mathrm{H}, 2 \times \mathrm{NCH}_{2} \mathrm{CH}_{2}\right), 1.54-1.49(\mathrm{~m}, 2 \mathrm{H}$, $\left.\mathrm{N}\left(\mathrm{CH}_{2}\right)_{2} \mathrm{CH}_{2}\right)$. Analytical data (NMR) in agreement with the literature. ${ }^{3}$

\section{1-[(4-(Trifluoromethyl)phenyl)thio]piperidine (1g)}

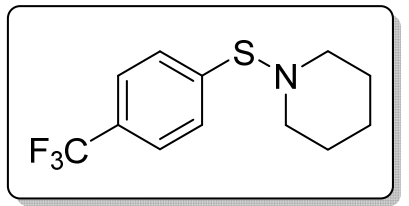

Prepared according to General Procedure A using 1,2-bis(4(trifluoromethyl)phenyl)disulfane ( $253 \mathrm{mg}, 0.72 \mathrm{mmol}, 1.0$ equiv). Purification by filtration through a plug of basic alumina (activity IV, $2 \mathrm{~cm}$ thick) using AcOEt $(300 \mathrm{~mL})$ afforded sulfenamide $1 \mathrm{~g}(113 \mathrm{mg}, 60 \%)$ as a colourless oil. ${ }^{1} \mathrm{H}$ NMR $\left(500 \mathrm{MHz}, \mathrm{CDCl}_{3}, \mathrm{ppm}\right) \delta 7.54(\mathrm{~d}, J=8.3 \mathrm{~Hz}, 2 \mathrm{H}, 2 \times \mathrm{Ar}-\mathrm{H}), 7.41(\mathrm{~d}$, $J=8.3 \mathrm{~Hz}, 2 \mathrm{H}, 2 \times \mathrm{Ar}-\mathrm{H}), 3.04-3.02\left(\mathrm{~m}, 4 \mathrm{H}, 2 \times \mathrm{NCH}_{2}\right), 1.68(\mathrm{q}, J=5.7 \mathrm{~Hz}$, $\left.4 \mathrm{H}, 2 \times \mathrm{NCH}_{2} \mathrm{CH}_{2}\right), 1.49-1.45\left(\mathrm{~m}, 2 \mathrm{H}, \mathrm{N}\left(\mathrm{CH}_{2}\right)_{2} \mathrm{CH}_{2}\right)$. Analytical data (NMR) in agreement with the in literature. ${ }^{1}$

\section{1-[(3-Methoxyphenyl)thio]pyrrolidine (1h)}

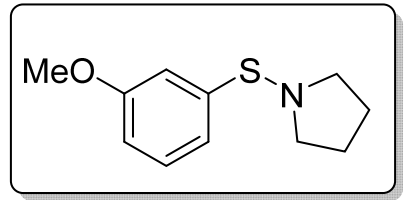

Prepared according to General Procedure A using 1,2-bis(3methoxyphenyl)disulfane ( $909 \mathrm{mg}, 3.26 \mathrm{mmol}, 1.0$ equiv). Purification by filtration through a plug of basic alumina (activity IV, $2 \mathrm{~cm}$ thick) using $\mathrm{CH}_{2} \mathrm{Cl}_{2}$ (300 mL) afforded sulfenamide $1 \mathrm{~h}(529 \mathrm{mg}, 77 \%)$ as a brown oil. ${ }^{1} \mathrm{H}$ NMR $\left(500 \mathrm{MHz}, \mathrm{CDCl}_{3}, \mathrm{ppm}\right) \delta 7.22(\mathrm{t}, J=8.1 \mathrm{~Hz}, 1 \mathrm{H}, \mathrm{Ar}-\mathrm{H}), 6.85-6.84(\mathrm{~m}, 2 \mathrm{H}, 2$ $\times \mathrm{Ar}-\mathrm{H}), 6.70-6.68(\mathrm{~m}, 1 \mathrm{H}, \mathrm{Ar}-\mathrm{H}), 3.80\left(\mathrm{~s}, 3 \mathrm{H}, \mathrm{OCH}_{3}\right) 3.16-3.13\left(\mathrm{~m}, 4 \mathrm{H}, 2 \times \mathrm{NCH}_{2}\right), 1.89-1.86(\mathrm{~m}, 4 \mathrm{H}$, $\left.2 \times \mathrm{NCH}_{2} \mathrm{CH}_{2}\right) .{ }^{13} \mathrm{C} \mathrm{NMR}\left(125 \mathrm{MHz}, \mathrm{CDCl}_{3}\right) \delta 160.0\left(\mathrm{Ar}-\mathrm{C}_{\mathrm{q}}\right), 141.7\left(\mathrm{Ar}-\mathrm{C}_{\mathrm{q}}\right), 129.6(\mathrm{Ar}-\mathrm{C}), 117.6(\mathrm{Ar}-\mathrm{C})$, 111.2 (Ar-C), $110.7(\mathrm{Ar}-\mathrm{C}) 55.2\left(2 \times \mathrm{NCH}_{2}\right), 55.2\left(\mathrm{Ar}-\mathrm{OCH}_{3}\right), 25.8\left(2 \times \mathrm{NCH}_{2} \mathrm{CH}_{2}\right)$. HRMS or elemental analysis data was not obtainable for compounds $1 \mathrm{~h}$ due to COVID lab closures.

\section{4-(Naphthalen-2-ylthio)morpholine (1i)}

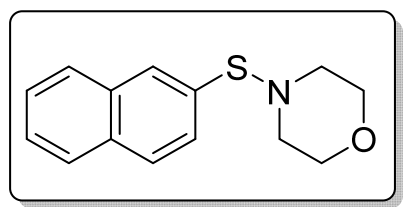

Prepared according to General Procedure A using 1,2-di(naphthalen-2yl)disulfane (316 mg, $0.99 \mathrm{mmol}, 1.0$ equiv). Purification by filtration through a plug of basic alumina (activity IV, $2 \mathrm{~cm}$ thick) using AcOEt (300 mL) afforded sulfenamide $1 \mathrm{i}(182 \mathrm{mg}, 75 \%)$ as a pink waxy solid. ${ }^{1} \mathrm{H}$ NMR $(500 \mathrm{MHz}$, $\left.\mathrm{CDCl}_{3}, \mathrm{ppm}\right) \delta 7.94(\mathrm{~s}, 1 \mathrm{H}, \mathrm{Ar}-\mathrm{H}), 7.84-7.81(\mathrm{~m}, 3 \mathrm{H}, \mathrm{Ar}-\mathrm{H}), 7.53-7.47(\mathrm{~m}$, $3 \mathrm{H}, \mathrm{Ar}-\mathrm{H}), 3.75-3.74\left(\mathrm{~m}, 4 \mathrm{H}, 2 \times \mathrm{OCH}_{2}\right) 3.04-3.02\left(\mathrm{~m}, 4 \mathrm{H}, 2 \times \mathrm{NCH}_{2}\right) \cdot{ }^{13} \mathrm{C} \mathrm{NMR}\left(125 \mathrm{MHz}, \mathrm{CDCl}_{3}\right) \delta$ $133.4\left(\mathrm{Ar}-\mathrm{C}_{\mathrm{q}}\right), 132.8\left(\mathrm{Ar}-\mathrm{C}_{\mathrm{q}}\right), 132.4\left(\mathrm{Ar}-\mathrm{C}_{\mathrm{q}}\right), 128.9(\mathrm{Ar}-\mathrm{C}), 127.8(\mathrm{Ar}-\mathrm{C}), 127.7(\mathrm{Ar}-\mathrm{C}), 126.5(\mathrm{Ar}-\mathrm{C})$, 126.3 (Ar-C), $67.7\left(2 \times \mathrm{OCH}_{2}\right), 56.2\left(2 \times \mathrm{NCH}_{2}\right)$. HRMS or elemental analysis data was not obtainable for compounds $1 \mathrm{~h}$ due to COVID lab closures.

\section{4-(Cyclohexylthio)morpholine (1j)}

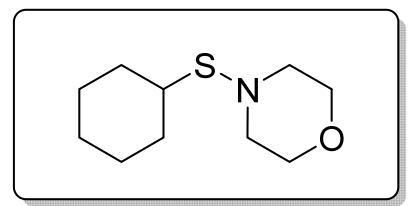

Prepared according to General Procedure A using 1,2-dicyclohexyldisulfane (1406 mg, $6.10 \mathrm{mmol}, 1.0$ equiv). Purification by filtration through a basic alumina (activity IV) plug (2 cm thick) with $\mathrm{CH}_{2} \mathrm{Cl}_{2}(300 \mathrm{~mL})$ afforded sulfenamide $1 \mathrm{j}$ (900 $\mathrm{mg}, 73 \%)$ as a colourless oil. ${ }^{1} \mathrm{H} \mathrm{NMR}\left(500 \mathrm{MHz}, \mathrm{CDCl}_{3}\right.$, ppm) ठ 3.66-3.64 (m, 4H, $\left.2 \times \mathrm{OCH}_{2}\right), 2.98-2.96\left(\mathrm{~m}, 4 \mathrm{H}, 2 \times \mathrm{NCH}_{2}\right), 2.88-$ $2.87(\mathrm{~m}, 1 \mathrm{H}, \mathrm{SCH}), 1.96-1.93\left(\mathrm{~m}, 2 \mathrm{H}, \mathrm{CH}_{2}\right), 1.77-1.74\left(\mathrm{~m}, 2 \mathrm{H}, \mathrm{CH}_{2}\right), 1.63-1.61\left(\mathrm{~m}, 2 \mathrm{H}, \mathrm{CH}_{2}\right), 1.34-1.30$ $\left(\mathrm{m}, 4 \mathrm{H}, 2 \times \mathrm{CH}_{2}\right) \cdot{ }^{13} \mathrm{C}$ NMR $\left(125 \mathrm{MHz}, \mathrm{CDCl}_{3}\right) \delta 67.7\left(2 \times \mathrm{OCH}_{2}\right), 58.1\left(2 \times \mathrm{NCH}_{2}\right), 47.6(\mathrm{SCH}), 32.1(2 \times$ $\left.\mathrm{CH}_{2}\right), 26.0\left(2 \times \mathrm{CH}_{2}\right), 25.8\left(\mathrm{CH}_{2}\right)$. Analytical data in agreement with the in literature. ${ }^{1 \mathrm{~b}}$ 


\section{Synthesis of $N$-mesyloxycarbamates $2 a$ and $2 b$}

\subsection{Synthesis of benzyl[(methylsulfonyl)oxy]carbamate (2a)}

Prepared using a modification of the procedure reported from Qin and co-workers. ${ }^{4}$

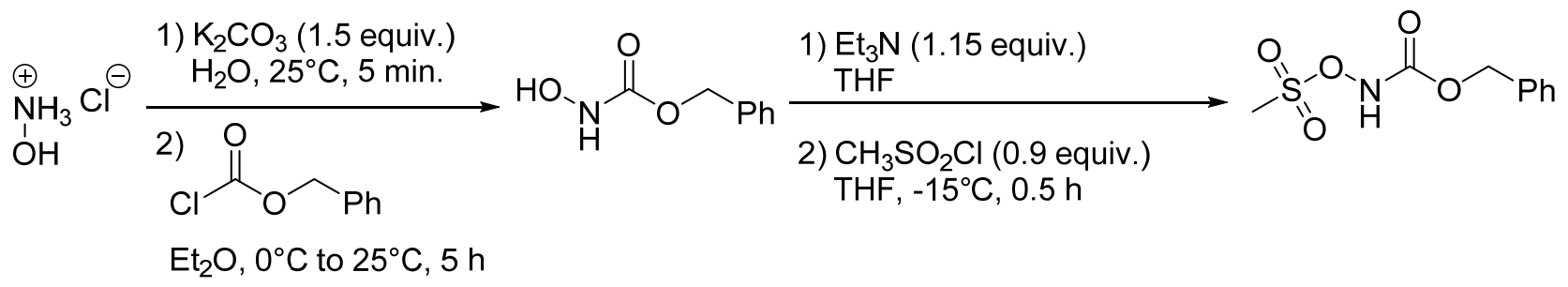

To a stirred solution of $\mathrm{K}_{2} \mathrm{CO}_{3}\left(1213 \mathrm{mg}, 8.80 \mathrm{mmol}, 1.5\right.$ equiv) in $\mathrm{H}_{2} \mathrm{O}$ (2 mL), hydroxylamine hydrochloride ( $405 \mathrm{mg}, 5.86 \mathrm{mmol}, 1.0$ equiv) was added in one portion. Subsequently, a solution of benzyl chloroformate (1000 mg, $5.86 \mathrm{mmol}$, 1 equiv) in $\mathrm{Et}_{2} \mathrm{O}(2 \mathrm{~mL})$ was added dropwise at $0^{\circ} \mathrm{C}$ and the reaction mixture was allowed to warm up to $25^{\circ} \mathrm{C}$ and stirred for $5 \mathrm{~h}$, and the solution acidified with concentrated hydrochloric acid. The resulting reaction mixture was extracted with $\mathrm{Et}_{2} \mathrm{O}(2 \times 10 \mathrm{~mL})$, the combined organic layers were dried $\left(\mathrm{Na}_{2} \mathrm{SO}_{4}\right)$, filtered and the solvent was concentrated under reduced pressure giving the crude $\mathrm{N}$-carbobenzyloxyhydroxylamine, which was used without further purification. A solution of methanesulfonyl chloride ( $476 \mathrm{mg}, 4.18 \mathrm{mmol}, 1.0$ equiv) in THF $(5.9 \mathrm{~mL})$ was added dropwise to a stirred solution of $N$-carbobenzyloxyhydroxylamine $(768 \mathrm{mg}, 4.60 \mathrm{mmol}, 1.1$ equiv) and triethylamine (482 $\mathrm{mg}, 4.76 \mathrm{mmol}, 1.15$ equiv) in THF $(2.7 \mathrm{~mL})$ at $-15^{\circ} \mathrm{C}$. After the addition, the solution was stirred at $-15^{\circ} \mathrm{C}$ for $0.5 \mathrm{~h}$. Subsequently, the precipitate triethylamine hydrochloride was filtered off and the filtrated solvent was concentrated under reduced pressure to give the crude product. The reaction crude was purified by flash chromatography on silica gel to afford benzyl((methylsulfonyl)oxy)carbamate $2 \mathrm{a}$ as a white solid (360 mg, 25\%). $\mathrm{R}_{f} 0.5$ (30\% AcOEt in Hexane).

\section{Benzyl((methylsulfonyl)oxy)carbamate (2a)}

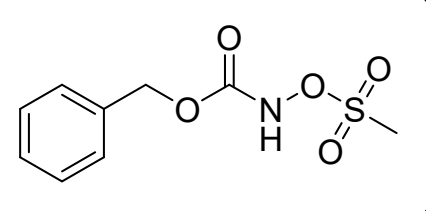

${ }^{1} \mathrm{H}$ NMR $\left(500 \mathrm{MHz}, \mathrm{CDCl}_{3}, \mathrm{ppm}\right) \delta 8.34(\mathrm{~s}, 1 \mathrm{H}, \mathrm{NH}), 7.41-7.34(\mathrm{~m}, 5 \mathrm{H}, \mathrm{Ar}-$ $\mathrm{H}), 5.24\left(\mathrm{~s}, 2 \mathrm{H}, \mathrm{OCH}_{2} \mathrm{Ph}\right), 3.13\left(\mathrm{~s}, 3 \mathrm{H}, \mathrm{O}_{3} \mathrm{SCH}_{3}\right) .{ }^{13} \mathrm{C} \mathrm{NMR}(125 \mathrm{MHz}$, $\left.\mathrm{CDCl}_{3}\right)$ ठ $155.8(\mathrm{NCOO}), 134.3\left(\mathrm{Ar}-\mathrm{C}_{\mathrm{q}}\right), 129.0(\mathrm{Ar}-\mathrm{C}), 128.8(\mathrm{Ar}-\mathrm{C}), 128.6$ $(\mathrm{Ar}-\mathrm{C}), 69.2\left(\mathrm{OCH}_{2} \mathrm{Ph}\right), 36.4\left(\mathrm{O}_{3} \mathrm{SCH}_{3}\right)$. Analytical data (NMR) in agreement with the literature. ${ }^{4}$ 


\subsection{Synthesis of isobutyl[(methylsulfonyl)oxy]carbamate (2b)}

Prepared in two steps adopting known procedures. ${ }^{5}$

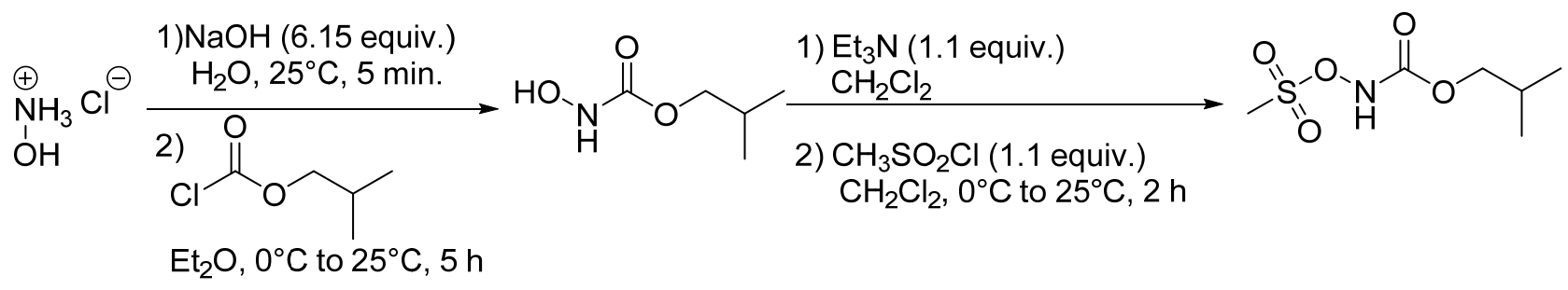

To a stirred solution of $\mathrm{NaOH}$ (1800 mg, $45 \mathrm{mmol}, 6.15$ equiv) in $\mathrm{H}_{2} \mathrm{O}$ (30 mL), hydroxylamine hydrochloride (2544 mg, $36.6 \mathrm{mmol}, 5.0$ equiv.) was added in one portion. Subsequently, isobutyl chloroformate (1000 mg, $7.32 \mathrm{mmol}, 1.0$ equiv) was added slowly at $0^{\circ} \mathrm{C}$ and the reaction mixture was allowed to warm up to $25^{\circ} \mathrm{C}$ and stirred for $2 \mathrm{~h}$, and the solution acidified with concentrated hydrochloric acid. The resulting reaction mixture was extracted with $\mathrm{Et}_{2} \mathrm{O}(3 \times 30 \mathrm{~mL})$, the combined organic layers were dried $\left(\mathrm{Na}_{2} \mathrm{SO}_{4}\right)$, filtered and the solvent was concentrated under reduced pressure giving the crude $\mathrm{N}$-carboisobutyloxyhydroxylamine, which was used without further purification. Methanesulfonyl chloride (875 mg, $7.64 \mathrm{mmol}, 1.1$ equiv.) was added dropwise to a stirred solution of $\mathrm{N}-$ carboisobutyloxyhydroxylamine ( $925 \mathrm{mg}, 6.95 \mathrm{mmol}, 1.0$ equiv.) and triethylamine $(773 \mathrm{mg}, 7.64 \mathrm{mmol}$, 1.1 equiv. ) in $\mathrm{CH}_{2} \mathrm{Cl}_{2}(35 \mathrm{~mL})$ at $0^{\circ} \mathrm{C}$. After the addition, the solution was allowed to warm up to $25^{\circ} \mathrm{C}$ and stirred for $2 \mathrm{~h}$. Subsequently, $\mathrm{H}_{2} \mathrm{O}(30 \mathrm{~mL})$ was added, and the aqueous mixture was extracted with $\mathrm{CH}_{2} \mathrm{Cl}_{2}(2 \times 25 \mathrm{~mL})$. The combined organic layers were dried $\left(\mathrm{Na}_{2} \mathrm{SO}_{4}\right)$, filtered and the solvent was concentrated under reduced pressure. The reaction crude was purified by flash chromatography on silica gel to afford isobutyl((methylsulfonyl)oxy)carbamate $\mathbf{2 b}$ as a white solid $(450 \mathrm{mg}, 29 \%)$. $\mathrm{R}_{f} 0.6(40 \%$ Hexane in $\mathrm{Et}_{2} \mathrm{O}$ ).

\section{Isobutyl((methylsulfonyl)oxy)carbamate (2b)}<smiles>CC(C)COC(=O)NOS(C)(=O)=O</smiles>

${ }^{1} \mathrm{H}$ NMR $\left(500 \mathrm{MHz}, \mathrm{CDCl}_{3}, \mathrm{ppm}\right) \delta 8.35(\mathrm{~s}, 1 \mathrm{H}, \mathrm{NH}), 4.02(\mathrm{~d}, J=6.7 \mathrm{~Hz}$, $2 \mathrm{H}, \mathrm{OCH}_{2} \mathrm{CH}$ ), $3.18\left(\mathrm{~s}, 3 \mathrm{H}, \mathrm{O}_{3} \mathrm{SCH}_{3}\right.$ ), 1.98 (hept, $J=6.7 \mathrm{~Hz}, 1 \mathrm{H}$, $\left.\mathrm{OCH}_{2} \mathrm{CH}\left(\mathrm{CH}_{3}\right)_{2}\right), 0.95\left(\mathrm{~d}, J=6.7 \mathrm{~Hz}, 6 \mathrm{H}, 2 \times \mathrm{CHCH}_{3}\right) \cdot{ }^{13} \mathrm{C} \mathrm{NMR}(125 \mathrm{MHz}$, $\left.\mathrm{CDCl}_{3}\right) \delta 156.3(\mathrm{NCOO}), 73.7\left(\mathrm{OCH}_{2} \mathrm{CH}\left(\mathrm{CH}_{3}\right)_{2}\right), 36.4\left(\mathrm{O}_{3} \mathrm{SCH}_{3}\right), 27.9$ $\left(\mathrm{OCH}_{2} \mathrm{CH}\left(\mathrm{CH}_{3}\right)_{2}\right), 18.9\left(2 \times \mathrm{OCH}_{2} \mathrm{CHCH}_{3}\right)$. HRMS (ESITOF) $\mathrm{m} / \mathrm{z}(\mathrm{M}+\mathrm{Na})^{+}$ calcd for $\mathrm{C}_{6} \mathrm{H}_{13} \mathrm{NNaO}_{5} \mathrm{~S} 234,0412$; found 234,0410. 


\section{Sulfinimidate esters}

\subsection{General Procedure B for sulfinimidate esters synthesis}

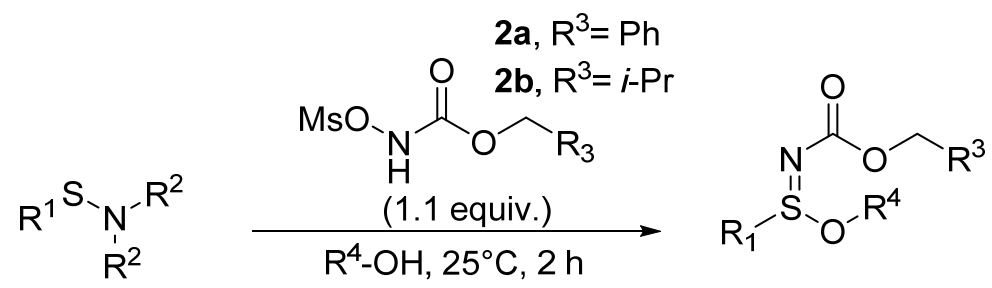

To a stirred solution of sulfenamide $(0.5 \mathrm{mmol}, 1.0$ equiv) in alcohol $(5 \mathrm{~mL}, 0.1 \mathrm{M})$, alkyl((methylsulfonyl)oxy)carbamate $(0.55 \mathrm{mmol}, 1.1$ equiv) was added in one portion, and the reaction mixture was stirred at $25^{\circ} \mathrm{C}$ for $2 \mathrm{~h}$. Subsequently, the solvent was concentrated under reduced pressure and $\mathrm{H}_{2} \mathrm{O}(15 \mathrm{~mL})$ was added to the crude. The aqueous mixture was extracted with $\mathrm{CH}_{2} \mathrm{Cl}_{2}(3 \times 15 \mathrm{~mL})$, the organic layers were collected, dried $\left(\mathrm{Na}_{2} \mathrm{SO}_{4}\right)$, filtered and the solvent removed under reduced pressure. The corresponding sulfinimidate ester was purified by flash chromatography on silica gel. In some cases no further purification was needed.

\subsection{Procedure $C$ for sulfinimidate ester 3a synthesis on $2 \mathrm{mmol}$ scale}

To a stirred solution of sulfenamide $1 \mathrm{a}(388 \mathrm{mg}, 2.0 \mathrm{mmol}, 1.0$ equiv) in ethanol (20 mL, $0.1 \mathrm{M})$, benzyl((methylsulfonyl)oxy)carbamate 2a (540 mg, $2.2 \mathrm{mmol}, 1.1$ equiv) was added in one portion, and the reaction mixture was stirred at $25^{\circ} \mathrm{C}$ for $2 \mathrm{~h}$. Subsequently, the solvent was concentrated under reduced pressure and $\mathrm{H}_{2} \mathrm{O}(50 \mathrm{~mL})$ was added to the crude. The aqueous mixture was extracted with $\mathrm{CH}_{2} \mathrm{Cl}_{2}(3 \times 50 \mathrm{~mL})$, the organic layers were collected, dried $\left(\mathrm{Na}_{2} \mathrm{SO}_{4}\right)$, filtered and the solvent removed under reduced pressure. The corresponding sulfinimidate ester $\mathbf{3 a}$ was obtained after purification by flash chromatography on silica gel in $87 \%$ of yield. 


\subsection{Characterization data for sulfinimidate esters 3a-s}

\section{Ethyl $N-[($ benzyloxy)carbonyl]phenylsulfinimidate (3a)}

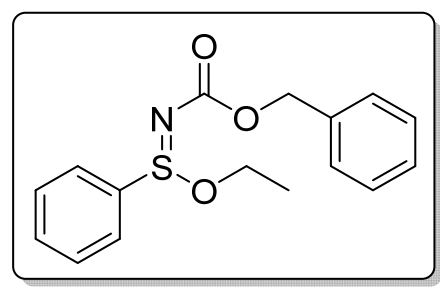
1.26 (t, $\left.\left.J=7.1 \mathrm{~Hz}, 3 \mathrm{H}, \mathrm{OCH}_{2} \mathrm{CH}_{3}\right).\right) .{ }^{13} \mathrm{C} \mathrm{NMR}\left(125 \mathrm{MHz}, \mathrm{CDCl}_{3}, \mathrm{ppm}\right) \delta 164.5$ (NCOO), $136.9\left(\mathrm{Ar}-\mathrm{C}_{\mathrm{q}}\right)$, 136.3 $\left(\mathrm{Ar}-\mathrm{C}_{\mathrm{q}}\right), 132.9(\mathrm{Ar}-\mathrm{C}), 129.5(\mathrm{Ar}-\mathrm{C}), 128.5(\mathrm{Ar}-\mathrm{C}), 128.4(\mathrm{Ar}-\mathrm{C}), 128.0(\mathrm{Ar}-\mathrm{C}), 127.7(\mathrm{Ar}-\mathrm{C}), 68.2$ $\left(\mathrm{OCH}_{2} \mathrm{Ph}\right), 63.2\left(\mathrm{OCH}_{2} \mathrm{CH}_{3}\right), 15.5\left(\mathrm{OCH}_{2} \mathrm{CH}_{3}\right)$. HRMS (ESITOF) $\mathrm{m} / \mathrm{z}(\mathrm{M}+\mathrm{Na})^{+}$calcd for $\mathrm{C}_{16} \mathrm{H}_{17} \mathrm{NNaO}_{3} \mathrm{~S}$ 326,0827 ; found 326,0819

\section{Methyl $\mathrm{N}-[($ isobutyloxy)carbonyl]phenylsulfinimidate (3b)}

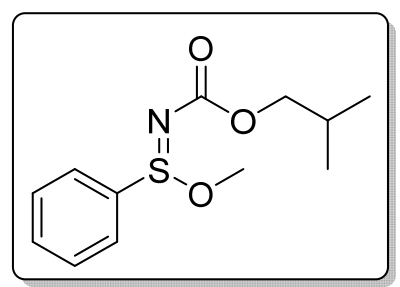

Prepared according to General Procedure B using sulfenamide 1a $(97 \mathrm{mg})$ in $\mathrm{MeOH}$, to afford sulfinimidate ester $\mathbf{3 b}$ as a waxy solid without further purification (126 mg, 99\%). IR (film)/ $/ \mathrm{cm}^{-1} 2959,1652,1446,1233,1067,955$, 893, 755. ${ }^{1} \mathrm{H}$ NMR (500 MHz, $\left.\mathrm{CDCl}_{3}, \mathrm{ppm}\right) \delta$ 7.93-7.91 (m, 2H, $\left.2 \times \mathrm{Ar}-\mathrm{H}\right)$, $7.59(\mathrm{t}, J=6.7 \mathrm{~Hz}, 1 \mathrm{H}, \mathrm{Ar}-\mathrm{H}), 7.55(\mathrm{t}, J=7.3 \mathrm{~Hz}, 2 \mathrm{H}, 2 \times \mathrm{Ar}-\mathrm{H}), 3.95(\mathrm{~d}, J=$ $6.7 \mathrm{~Hz}, 2 \mathrm{H}, \mathrm{OCH}_{2} \mathrm{CH}$ ), 3.52 (s, $3 \mathrm{H}, \mathrm{SOCH}_{3}$ ), 2.02 (hept, $J=6.7 \mathrm{~Hz}, 1 \mathrm{H}$, $\left.\mathrm{OCH}_{2} \mathrm{CH}\left(\mathrm{CH}_{3}\right)_{2}\right), 0.96\left(\mathrm{~d}, \mathrm{~J}=6.7 \mathrm{~Hz}, 6 \mathrm{H}, 2 \times \mathrm{OCH}_{2} \mathrm{CHCH}_{3}\right) \cdot{ }^{13} \mathrm{C}$ NMR $(125$ $\left.\mathrm{MHz}, \mathrm{CDCl}_{3}\right) \delta 164.4$ (NCOO), $135.3\left(\mathrm{Ar}-\mathrm{C}_{\mathrm{q}}\right), 132.9$ (Ar-C), 129.4 (2 × Ar-C), 127.8 (2 × Ar-C), 72.7 $\left(\mathrm{OCH}_{2} \mathrm{CH}\right), 50.9\left(\mathrm{SOCH}_{3}\right), 28.0\left(\mathrm{OCH}_{2} \mathrm{CH}\left(\mathrm{CH}_{3}\right)_{2}\right), 19.2\left(2 \times \mathrm{OCH}_{2} \mathrm{CH}\left(\mathrm{CH}_{3}\right)_{2}\right) . \mathrm{HRMS}$ (ESITOF) $\mathrm{m} / \mathrm{z}$ $(\mathrm{M}+\mathrm{Na})^{+}$calcd for $\mathrm{C}_{12} \mathrm{H}_{17} \mathrm{NNaO}_{3} \mathrm{~S} 278,0827$; found 278,0829 .

\section{Propyl N-[(isobutyloxy)carbonyl]phenylsulfinimidate (3c)}

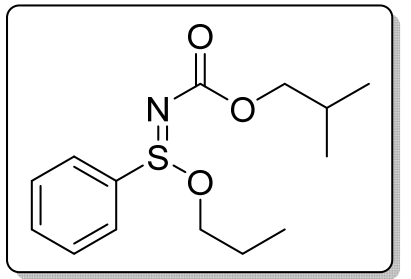

Prepared according to General Procedure B using sulfenamide 1a (97 mg) in 1-propanol, to afford sulfinimidate ester $3 \mathrm{c}$ as a waxy solid without further purification (128 mg, 90\%). IR (film) $/ \mathrm{cm}^{-1} 2962,2879,1650,1468,1233,1083$, 891, 789. ${ }^{1} \mathrm{H}$ NMR $\left(500 \mathrm{MHz}, \mathrm{CDCl}_{3}, \mathrm{ppm}\right) \delta 7.94-7.92(\mathrm{~m}, 2 \mathrm{H}, 2 \times \mathrm{Ar}-\mathrm{H})$, 7.60-7.53 $(\mathrm{m}, 3 \mathrm{H}, 3 \times \mathrm{Ar}-\mathrm{H}), 4.12$ and $3.67(2 \times \mathrm{dt}, J=9.8,6.6 \mathrm{~Hz}, \mathrm{ABX}$ system, $\left.2 \mathrm{H}, 2 \times \mathrm{OCHHCH}_{2}\right), 3.97$ and $3.94(2 \times \mathrm{dd}, \mathrm{J}=9.0,5.3 \mathrm{~Hz}, 2 \mathrm{H}, \mathrm{ABX}$ system, $2 \times \mathrm{OCHHCH}\left(\mathrm{CH}_{3}\right)_{2}$ ), 2.03 (hept, $J=6.7 \mathrm{~Hz}, 1 \mathrm{H}, \mathrm{OCH}_{2} \mathrm{CH}\left(\mathrm{CH}_{3}\right)_{2}$ ), 1.66-1.61 (m, 2H, OCH $\left.\mathrm{CH}_{2} \mathrm{CH}_{3}\right), 0.97\left(\mathrm{~d}, J=6.7 \mathrm{~Hz}, 6 \mathrm{H}, 2 \times \mathrm{CH}_{2} \mathrm{CH}\left(\mathrm{CH}_{3}\right)\right), 0.89$ (t, $J=7.4 \mathrm{~Hz}, 3 \mathrm{H}$,

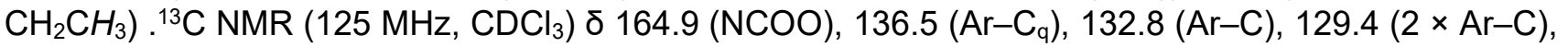
$127.8(2 \times \mathrm{Ar}-\mathrm{C}), 72.8\left(\mathrm{OCH}_{2} \mathrm{CH}\right), 68.2\left(\mathrm{SOCH}_{2} \mathrm{CH}_{2}\right), 28.1\left(\mathrm{OCH}_{2} \mathrm{CH}\left(\mathrm{CH}_{3}\right)_{2}\right), 23.1\left(\mathrm{SOCH}_{2} \mathrm{CH}_{2} \mathrm{CH}_{3}\right), 19.4$ $\left(2 \times \mathrm{CHCH}_{3}\right), 10.3\left(\mathrm{OCH}_{2} \mathrm{CH}_{2} \mathrm{CH}_{3}\right)$. HRMS (ESITOF) $\mathrm{m} / \mathrm{z}(\mathrm{M}+\mathrm{Na})^{+}$calcd for $\mathrm{C}_{14} \mathrm{H}_{21} \mathrm{NNaO}_{3} \mathrm{~S} 306,1140$; found 306,1143 . 


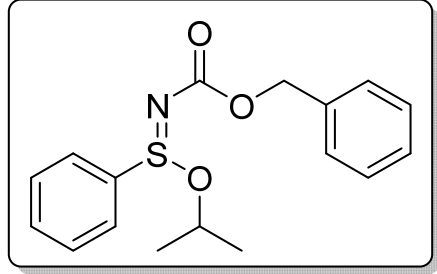

Prepared according to General Procedure B using sulfenamide 1a $(97 \mathrm{mg})$ in 2-propanol, to afford sulfinimidate ester $\mathbf{3 d}$ as a waxy solid $(159 \mathrm{mg}$, 85\%). $\mathrm{R}_{f} 0.3$ (50\% $\mathrm{Et}_{2} \mathrm{O}$ in Hexane). IR (film)/cm-1 2977, 1649, 1447, 1232, 1094, 890, 727. ${ }^{1} \mathrm{H}$ NMR $\left(500 \mathrm{MHz}, \mathrm{CDCl}_{3}, \mathrm{ppm}\right) \delta 7.91-7.89(\mathrm{~m}, 2 \mathrm{H}, 2 \times$ Ar-H), 7.57-7.54 (m, 1H, Ar-H), 7.53-7.50 (m, 2H, $2 \times \mathrm{Ar}-\mathrm{H}), 7.42(\mathrm{~d}, \mathrm{~J}=$ $7.2 \mathrm{~Hz}, 2 \mathrm{H}, 2 \times \mathrm{Ar}-\mathrm{H}), 7.34-7.31(\mathrm{~m}, 2 \mathrm{H}, \mathrm{Ar}-\mathrm{H}), 7.29-7.27(\mathrm{~m}, 1 \mathrm{H}, \mathrm{Ar}-\mathrm{H})$, 5.22 and $5.20(2 \times \mathrm{d}, J=12.9 \mathrm{~Hz}, \mathrm{AB}$ system, $2 \mathrm{H}, 2 \times \mathrm{OCH} H \mathrm{Ph}), 4.80$ (hept, $\left.J=6.2 \mathrm{~Hz}, 1 \mathrm{H}, \mathrm{OCH}\left(\mathrm{CH}_{3}\right)_{2}\right), 1.37\left(\mathrm{~d}, J=6.2 \mathrm{~Hz}, 3 \mathrm{H}, \mathrm{OCHCH}_{3}\right), 1.20(\mathrm{~d}, J=6.2 \mathrm{~Hz}, 3 \mathrm{H}$, $\left.\mathrm{OCHCH}_{3}\right) \cdot{ }^{13} \mathrm{C}$ NMR $\left(125 \mathrm{MHz}, \mathrm{CDCl}_{3}\right) \delta 164.8(\mathrm{NCOO}), 137.3\left(\mathrm{Ar}-\mathrm{C}_{\mathrm{q}}\right), 136.9\left(\mathrm{Ar}-\mathrm{C}_{\mathrm{q}}\right), 132.7(\mathrm{Ar}-\mathrm{C})$, $129.3(2 \times \mathrm{Ar}-\mathrm{C}), 128.4(2 \times \mathrm{Ar}-\mathrm{C}), 128.3$ (2 $\times \mathrm{Ar}-\mathrm{C}), 127.9(\mathrm{Ar}-\mathrm{C}), 127.4(2 \times \mathrm{Ar}-\mathrm{C}), 75.3\left(\mathrm{OCH}\left(\mathrm{CH}_{3}\right)_{2}\right)$, $68.1\left(\mathrm{OCH}_{2} \mathrm{Ph}\right), 23.7$ and $23.4\left(2 \times \mathrm{OCHCH}_{3}\right)$. HRMS (ESITOF) $\mathrm{m} / \mathrm{z}(\mathrm{M}+\mathrm{Na})^{+}$calcd for $\mathrm{C}_{17} \mathrm{H}_{19} \mathrm{NNaO}_{3} \mathrm{~S}$ 340,0983 ; found 340,0983 .

\section{Butyl N-[(isobutyloxy)carbonyl]phenylsulfinimidate (3e)}

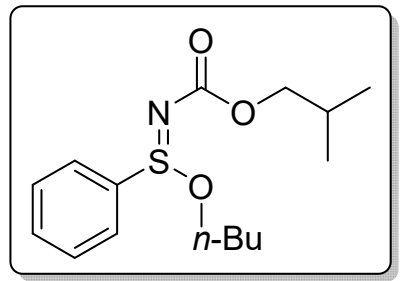

Prepared according to General Procedure B using sulfenamide 1a $(97 \mathrm{mg})$ in 1-butanol, to afford sulfinimidate ester $3 e$ as a waxy solid (127 mg, $90 \%) . R_{f}$ 0.5 (50\% $\mathrm{Et}_{2} \mathrm{O}$ in Hexane). IR (film)/ $\mathrm{cm}^{-1} 2959,1652,1374,1234,890,688$. ${ }^{1} \mathrm{H}$ NMR $\left(500 \mathrm{MHz}, \mathrm{CDCl}_{3}, \mathrm{ppm}\right) \delta 7.92(\mathrm{~d}, J=7.4 \mathrm{~Hz}, 2 \mathrm{H}, 2 \times \mathrm{Ar}-\mathrm{H}), 7.60$ $7.53(\mathrm{~m}, 3 \mathrm{H}, 3 \times \mathrm{Ar}-\mathrm{H}), 4.16$ and $3.70(2 \times \mathrm{dt}, J=9.9,6.6 \mathrm{~Hz}, \mathrm{ABX}$ system, $\left.2 \mathrm{H}, 2 \times \mathrm{OCHHCH}_{2} \mathrm{CH}_{2}\right), 3.97$ and $3.94(2 \times \mathrm{dd}, J=10.1,6.5 \mathrm{~Hz}, \mathrm{ABX}$ system, $\left.2 \mathrm{H}, 2 \times \mathrm{OCHHCH}\left(\mathrm{CH}_{3}\right)_{2}\right), 2.02$ (hept, $\left.J=6.7 \mathrm{~Hz}, 1 \mathrm{H}, \mathrm{OCH}_{2} \mathrm{CH}\left(\mathrm{CH}_{3}\right)_{2}\right), 1.62-$ $1.56\left(\mathrm{~m}, 2 \mathrm{H}, \mathrm{OCH}_{2} \mathrm{CH}_{2} \mathrm{CH}_{2}\right), 1.36-1.29\left(\mathrm{~m}, 2 \mathrm{H}, \mathrm{CH}_{2} \mathrm{CH}_{2} \mathrm{CH}_{3}\right), 0.97\left(\mathrm{~d}, J=6.7 \mathrm{~Hz}, 6 \mathrm{H}, 2 \times \mathrm{CHCH}_{3}\right), 0.86$ $\left(\mathrm{t}, J=7.4 \mathrm{~Hz}, 3 \mathrm{H}, \mathrm{CH}_{2} \mathrm{CH}_{3}\right) .{ }^{13} \mathrm{C}$ NMR $\left(125 \mathrm{MHz}, \mathrm{CDCl}_{3}\right) \delta 164.8$ (NCOO), $136.5\left(\mathrm{Ar}-\mathrm{C}_{\mathrm{q}}\right), 132.8(\mathrm{Ar}-\mathrm{C})$, $129.4(2 \times \mathrm{Ar}-\mathrm{C}), 127.8(2 \times \mathrm{Ar}-\mathrm{C}), 72.8\left(\mathrm{OCH}_{2} \mathrm{CH}\right), 66.4\left(\mathrm{OCH}_{2} \mathrm{CH}_{2}\right), 31.7\left(\mathrm{OCH}_{2} \mathrm{CH}_{2} \mathrm{CH}_{2}\right), 28.1$ $\left(\mathrm{OCH}_{2} \mathrm{CH}\left(\mathrm{CH}_{3}\right)_{2}\right), 19.4\left(2 \times \mathrm{CH}(\mathrm{CH} 3)_{2}\right), 19.0\left(\mathrm{CH}_{2} \mathrm{CH}_{2} \mathrm{CH}_{3}\right), 13.7\left(\mathrm{CH}_{2} \mathrm{CH}_{2} \mathrm{CH}_{3}\right)$. ). HRMS (ESITOF) m/z $(\mathrm{M}+\mathrm{Na})^{+}$calcd for $\mathrm{C}_{15} \mathrm{H}_{23} \mathrm{NNaO}_{3} \mathrm{~S} 320,1296$; found 320,1299 .

\section{Hexyl $\mathrm{N}$-[(isobutyloxy)carbonyl]phenylsulfinimidate (3f)}

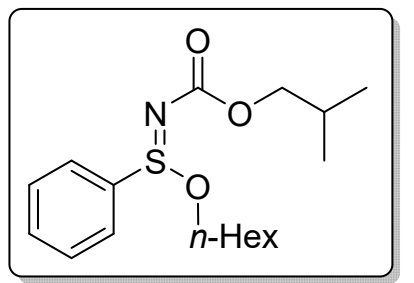

Prepared according to General Procedure B using sulfenamide 1a $(97 \mathrm{mg})$ in 1-hexanol, to afford sulfinimidate ester $3 f$ as a waxy solid (124 $\mathrm{mg}, 73 \%)$. $\mathrm{R}_{f}$ 0.6 (20\% Hexane in $\left.\mathrm{Et}_{2} \mathrm{O}\right)$. IR (film)/ $/ \mathrm{cm}^{-1} 2956,1652,1374,1233,972,688$. ${ }^{1} \mathrm{H}$ NMR $\left(500 \mathrm{MHz}, \mathrm{CDCl}_{3}, \mathrm{ppm}\right) \delta 7.93-7.91(\mathrm{~m}, 2 \mathrm{H}, 2 \times \mathrm{Ar}-\mathrm{H}), 7.58-7.52$ $(\mathrm{m}, 3 \mathrm{H}, 3 \times \mathrm{Ar}-\mathrm{H}), 4.14$ and $3.69(2 \times \mathrm{dt}, J=9.9,6.7 \mathrm{~Hz}, \mathrm{ABX}$ system, $2 \mathrm{H}, 2$ $\left.\times \mathrm{OCHHCH}_{2}\right), 3.96$ and $3.94(2 \times \mathrm{dd}, J=10.5,6.8 \mathrm{~Hz}, \mathrm{ABX}$ system, $2 \mathrm{H}, 2 \times$ $\left.\mathrm{OCHHCH}\left(\mathrm{CH}_{3}\right)_{2}\right), 2.02$ (hept, $\left.J=6.7 \mathrm{~Hz}, 1 \mathrm{H}, \mathrm{OCH}_{2} \mathrm{CH}\left(\mathrm{CH}_{3}\right)_{2}\right), 1.63-1.57(\mathrm{~m}$, $\left.2 \mathrm{H}, \mathrm{OCH}_{2} \mathrm{CH}_{2} \mathrm{CH}_{2}\right), 1.29-1.18\left(\mathrm{~m}, 6 \mathrm{H}, \mathrm{CH}_{2}\left(\mathrm{CH}_{2}\right)_{3} \mathrm{CH}_{3}\right), 0.97\left(\mathrm{~d}, J=6.7 \mathrm{~Hz}, 6 \mathrm{H}, 2 \times \mathrm{CHCH}_{3}\right), 0.84(\mathrm{t}, J=$ $\left.7.0 \mathrm{~Hz}, 3 \mathrm{H}, \mathrm{O}\left(\mathrm{CH}_{2}\right)_{5} \mathrm{CH}_{3}\right) .{ }^{13} \mathrm{C} \mathrm{NMR}\left(125 \mathrm{MHz}, \mathrm{CDCl}_{3}\right) \delta 164.8(\mathrm{NCOO}), 136.5\left(\mathrm{Ar}-\mathrm{C}_{\mathrm{q}}\right), 132.8(\mathrm{Ar}-\mathrm{C})$, $129.4(2 \times \mathrm{Ar}-\mathrm{C}), 127.8(2 \times \mathrm{Ar}-\mathrm{C}), 72.8\left(\mathrm{OCH}_{2} \mathrm{CH}\right), 66.7\left(\mathrm{OCH}_{2} \mathrm{CH}_{2}\right), 31.4\left(\mathrm{CH}_{2}\right), 29.7\left(\mathrm{CH}_{2}\right), 28.1$ $\left(\mathrm{OCH}_{2} \mathrm{CH}\left(\mathrm{CH}_{3}\right)_{2}\right), 25.4\left(\mathrm{CH}_{2}\right), 22.6\left(\mathrm{CH}_{2} \mathrm{CH}_{2} \mathrm{CH}_{3}\right), 19.4\left(2 \times \mathrm{CHCH}_{3}\right), 14.1\left(\mathrm{CH}_{2} \mathrm{CH}_{2} \mathrm{CH}_{3}\right) . \mathrm{HRMS}$ (ESITOF) $\mathrm{m} / \mathrm{z}(\mathrm{M}+\mathrm{Na})^{+}$calcd for $\mathrm{C}_{17} \mathrm{H}_{27} \mathrm{NNaO}_{3} \mathrm{~S} 348,1609$; found 348,1612 .

\section{Methyl $\mathrm{N}$-[(benzyloxy)carbonyl]-4-methoxyphenylsulfinimidate $(3 \mathrm{~g})$}

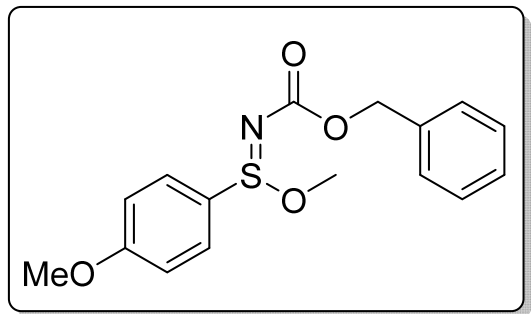

Prepared according to General Procedure B using sulfenamide 1b $(112 \mathrm{mg}$ ) in $\mathrm{MeOH}$, to afford sulfinimidate ester $\mathbf{3 g}$ as a waxy solid (128 mg, 80\%). $R_{f} 0.65$ (40\% Hexane in AcOEt). IR (film)/cm ${ }^{-1} 2940$, 1652, 1591, 1455, 1228, 1085, 890, 749. ${ }^{1} \mathrm{H}$ NMR $\left(500 \mathrm{MHz}, \mathrm{CDCl}_{3}\right.$, ppm) $\delta 7.87-7.84(\mathrm{~m}, 2 \mathrm{H}, 2 \times \mathrm{Ar}-\mathrm{H}), 7.43(\mathrm{~d}, J=7.5 \mathrm{~Hz}, 2 \mathrm{H}, 2 \times \mathrm{Ar}-$ H) 7.36-7.33 (m, 2H, $2 \times \mathrm{Ar}-\mathrm{H}), 7.30-7.27(\mathrm{~m}, 1 \mathrm{H}, \mathrm{Ar}-\mathrm{H}), 7.04-7.01$ $(\mathrm{m}, 2 \mathrm{H}, 2 \times \mathrm{Ar}-\mathrm{H}), 5.23$ and $5.19(2 \times \mathrm{d}, J=12.3 \mathrm{~Hz}, \mathrm{AB}$ system, $2 \mathrm{H}$, $2 \times \mathrm{OCH} H \mathrm{Ph}), 3.86\left(\mathrm{~s}, 3 \mathrm{H}, \mathrm{Ar}-\mathrm{OCH}_{3}\right), 3.47\left(\mathrm{~s}, 3 \mathrm{H}, \mathrm{SOCH}_{3}\right) .{ }^{13} \mathrm{C} \mathrm{NMR}$ 
$\left(125 \mathrm{MHz}, \mathrm{CDCl}_{3}, \mathrm{ppm}\right) \delta 164.2(\mathrm{NCOO}), 163.5\left(\mathrm{Ar}-\mathrm{C}_{\mathrm{q}}\right), 136.9\left(\mathrm{Ar}-\mathrm{C}_{\mathrm{q}}\right), 129.9(2 \times \mathrm{Ar}-\mathrm{C}), 128.5(2 \times \mathrm{Ar}-$ C), $128.4(2 \times \mathrm{Ar}-\mathrm{C}), 128.0(\mathrm{Ar}-\mathrm{C}), 126.0\left(\mathrm{Ar}-\mathrm{C}_{\mathrm{q}}\right), 114.9(2 \times \mathrm{Ar}-\mathrm{C}), 68.2\left(\mathrm{COOCH}_{2}\right), 55.8\left(\mathrm{Ar}-\mathrm{OCH}_{3}\right)$, $50.4\left(\mathrm{SOCH}_{3}\right)$. HRMS (ESITOF) m/z (M+Na) calcd for $\mathrm{C}_{16} \mathrm{H}_{17} \mathrm{NNaO}_{4} \mathrm{~S} 342,0773$; found 342,0784.

\section{Ethyl $\mathrm{N}$-((benzyloxy)carbonyl)-4-methoxyphenylsulfinimidate (3h)}

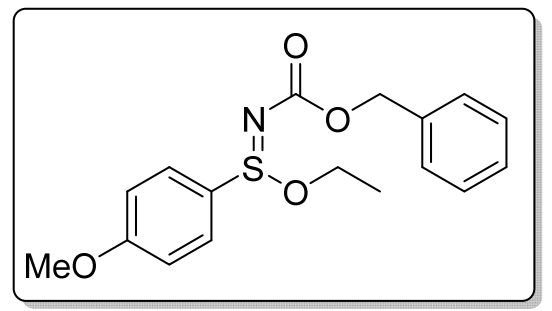

Prepared according to General Procedure B using sulfenamide 1b $(112 \mathrm{mg})$ in $\mathrm{EtOH}$, to afford sulfinimidate ester $\mathbf{3 h}$ as a waxy solid (122 mg, 73\%). $\mathrm{R}_{f} 0.6$ (50\% AcOEt in Hexane). IR (film)/cm ${ }^{-1} 2982$, $1726,1651,1591,1494,1233,868,749 .{ }^{1} \mathrm{H}$ NMR $\left(500 \mathrm{MHz}, \mathrm{CDCl}_{3}\right.$, ppm) $\delta 7.87-7.84(\mathrm{~m}, 2 \mathrm{H}, 2 \times \mathrm{Ar}-\mathrm{H}), 7.43(\mathrm{~d}, J=7.4 \mathrm{~Hz}, 2 \mathrm{H}, 2 \times \mathrm{Ar}-$ $\mathrm{H}), 7.35-7.32(\mathrm{~m}, 2 \mathrm{H}, 2 \times \mathrm{Ar}-\mathrm{H}), 7.30-7.27(\mathrm{~m}, 1 \mathrm{H}, \mathrm{Ar}-\mathrm{H}), 7.03-7.00$ $(\mathrm{m}, 2 \mathrm{H}, 2 \times \mathrm{Ar}-\mathrm{H}), 5.24$ and $5.21(2 \times \mathrm{d}, J=12.3 \mathrm{~Hz}, \mathrm{AB}$ system, $2 \mathrm{H}$, $2 \times \mathrm{OCH} H \mathrm{Ph}), 4.22$ and $3.81(2 \times \mathrm{dq}, J=10.0,7.5 \mathrm{~Hz}, \mathrm{ABX}$ system, $2 \mathrm{H}, 2 \times \mathrm{SOCHHCH}$ ), $3.86(\mathrm{~s}, 3 \mathrm{H}$, $\mathrm{OCH}_{3}$ ), 1.23 (t, $\left.J=7.1 \mathrm{~Hz}, 3 \mathrm{H}, \mathrm{SOCH}_{2} \mathrm{CH}_{3}\right) .{ }^{13} \mathrm{C} \mathrm{NMR}\left(125 \mathrm{MHz}, \mathrm{CDCl}_{3}, \mathrm{ppm}\right) \delta 164.5$ (NCOO), 163.4 $\left(\mathrm{Ar}-\mathrm{C}_{\mathrm{q}}\right), 137.0\left(\mathrm{Ar}-\mathrm{C}_{\mathrm{q}}\right), 129.7(2 \times \mathrm{Ar}-\mathrm{C}), 128.5(2 \times \mathrm{Ar}-\mathrm{C}), 128.4(2 \times \mathrm{Ar}-\mathrm{C}), 128.0(\mathrm{Ar}-\mathrm{C}), 127.3(\mathrm{Ar}-$ $\left.\mathrm{C}_{\mathrm{q}}\right), 114.8(2 \times \mathrm{Ar}-\mathrm{C}), 68.1\left(\mathrm{OCH}_{2} \mathrm{Ph}\right), 62.3\left(\mathrm{SOCH}_{2} \mathrm{CH}_{3}\right), 55.8\left(\mathrm{Ar}-\mathrm{OCH}_{3}\right), 15.5\left(\mathrm{SOCH}_{2} \mathrm{CH}_{3}\right) . \mathrm{HRMS}$ (ESITOF) $\mathrm{m} / \mathrm{z}(\mathrm{M}+\mathrm{Na})^{+}$calcd for $\mathrm{C}_{17} \mathrm{H}_{19} \mathrm{NNaO}_{4} \mathrm{~S} 356,0932$; found 356,0932 .

\section{Ethyl $N$-((benzyloxy)carbonyl)-4-chlorophenylsulfinimidate (3i)}

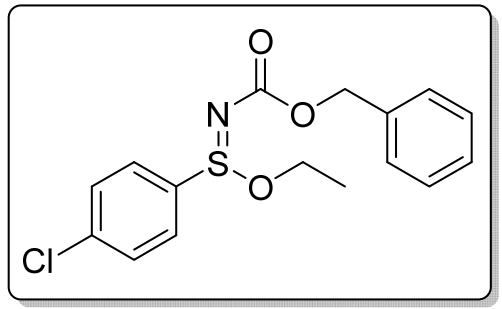

Prepared according to General Procedure B using sulfenamide 1c (114 $\mathrm{mg})$ in $\mathrm{EtOH}$, to afford sulfinimidate ester $3 \mathbf{i}$ as a waxy solid $(118 \mathrm{mg}$, $70 \%$ ). $\mathrm{R}_{f} 0.4$ (30\% Hexane in $\mathrm{Et}_{2} \mathrm{O}$ ). IR (film) $/ \mathrm{cm}^{-1}$ 2982, 1653, 1574 , 1376, 1231, 1082, 867, 759. ${ }^{1} \mathrm{H}$ NMR $\left(500 \mathrm{MHz}, \mathrm{CDCl}_{3}, \mathrm{ppm}\right) \delta 7.87-$ $7.84(\mathrm{~m}, 2 \mathrm{H}, 2 \times \mathrm{Ar}-\mathrm{H}), 7.52-7.50(\mathrm{~m}, 2 \mathrm{H}, 2 \times \mathrm{Ar}-\mathrm{H}) 7.43(\mathrm{~d}, J=7.3$ $\mathrm{Hz}, 2 \mathrm{H}, 2 \times \mathrm{Ar}-\mathrm{H}), 7.36-7.28(\mathrm{~m}, 3 \mathrm{H}, 3 \times \mathrm{Ar}-\mathrm{H}), 5.22$ and $5.19(2 \times \mathrm{d}$, $J=12.2 \mathrm{~Hz}, \mathrm{AB}$ system, $2 \mathrm{H}, 2 \times \mathrm{OCH} H \mathrm{Ph}), 4.21$ and $3.80(2 \times \mathrm{dq}, J=$ $10.0 \mathrm{~Hz}, 7.1,2 \mathrm{H}, 2 \times \mathrm{CH}, \mathrm{SOCHHCH}), 1.26\left(\mathrm{t}, J=7.1 \mathrm{~Hz}, 3 \mathrm{H}, \mathrm{SOCH}_{2} \mathrm{CH}_{3}\right) .{ }^{13} \mathrm{C} \mathrm{NMR}\left(125 \mathrm{MHz}, \mathrm{CDCl}_{3}\right.$, ppm) $\delta 164.2(\mathrm{NCOO}), 139.4\left(\mathrm{Ar}-\mathrm{C}_{\mathrm{q}}\right), 136.6\left(\mathrm{Ar}-\mathrm{C}_{\mathrm{q}}\right), 134.7(2 \times \mathrm{Ar}-\mathrm{C}), 129.6(2 \times \mathrm{Ar}-\mathrm{C}), 129.0(2 \times \mathrm{Ar}-$ C), $128.4(2 \times \mathrm{Ar}-\mathrm{C}), 128.3(2 \times \mathrm{Ar}-\mathrm{C}), 128.0(\mathrm{Ar}-\mathrm{C}), 68.2\left(\mathrm{OCH}_{2} \mathrm{Ph}\right), 63.2\left(\mathrm{SOCH}_{2} \mathrm{CH}_{3}\right), 15.4$ $\left(\mathrm{SOCH}_{2} \mathrm{CH}_{3}\right)$. HRMS (ESITOF) m/z (M+Na) $)^{+}$calcd for $\mathrm{C}_{16} \mathrm{H}_{16} \mathrm{CINNaO}_{3} \mathrm{~S} 360,0437$; found 360,0437.

\section{Methyl $N-[($ benzyloxy)carbonyl]-4-fluorophenylsulfinimidate (3j)}

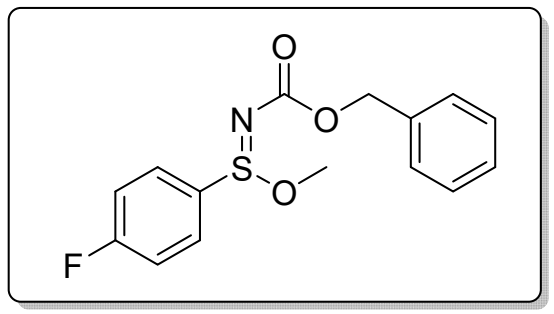

Prepared according to General Procedure B using sulfenamide 1d (106 $\mathrm{mg}$ ) in $\mathrm{MeOH}$, to afford sulfinimidate ester $\mathbf{3} \mathbf{j}$ as a colourless solid (95 mg, 62\%). $\mathrm{R}_{f} 0.55$ (40\% AcOEt in Hexane). IR (film) $/ \mathrm{cm}^{-1}$ 2929, 1655, 1589, 1491, 1375, 1230, 953, 737. ${ }^{1} \mathrm{H}$ NMR $(500 \mathrm{MHz}$, $\left.\mathrm{CDCl}_{3}, \mathrm{ppm}\right) \delta 7.96-7.93(\mathrm{~m}, 2 \mathrm{H}, 2 \times \mathrm{Ar}-\mathrm{H}), 7.43(\mathrm{~d}, J=7.3 \mathrm{~Hz}, 2 \mathrm{H}$, $2 \times \mathrm{Ar}-\mathrm{H}), 7.35(\mathrm{t}, J=7.3 \mathrm{~Hz}, 2 \mathrm{H}, 2 \times \mathrm{Ar}-\mathrm{H}),), 7.30(\mathrm{t}, J=7.3 \mathrm{~Hz}$, $1 \mathrm{H}, \mathrm{Ar}-\mathrm{H}), 7.27-7.22(\mathrm{~m}, 2 \mathrm{H}, \mathrm{Ar}-\mathrm{H}), 5.23$ and $5.20(2 \times \mathrm{d}, J=12.3$ $\mathrm{Hz}, \mathrm{AB}$ system, $2 \mathrm{H}, 2 \times \mathrm{OCHHPh}), 3.52\left(\mathrm{~s}, 3 \mathrm{H}, \mathrm{SOCH}_{3}\right) .{ }^{19} \mathrm{~F} \mathrm{NMR}\left(470 \mathrm{MHz}, \mathrm{CDCl}_{3}\right) \delta-104.85$ (tt, ${ }^{3} \mathrm{~F}_{\mathrm{F}-\mathrm{H}}$ $\left.=8.1,{ }^{4} J_{\mathrm{F}-\mathrm{H}}=5.0 \mathrm{~Hz}\right) \cdot{ }^{13} \mathrm{C} \mathrm{NMR}\left(125 \mathrm{MHz}, \mathrm{CDCl}_{3}, \mathrm{ppm}\right) \delta 165.6\left(\mathrm{~d},{ }^{1} \mathrm{~J}_{\mathrm{C}-\mathrm{F}}=255.5 \mathrm{~Hz}, \mathrm{Ar}-\mathrm{C}_{\mathrm{q}}\right), 164.1$ (NCOO), $136.7\left(\mathrm{Ar}-\mathrm{C}_{\mathrm{q}}\right), 130.9\left(\mathrm{~d},{ }^{4} \mathrm{~J} \mathrm{C}-\mathrm{F}=3.1 \mathrm{~Hz}, \mathrm{Ar}-\mathrm{C}_{\mathrm{q}}\right), 130.55\left(\mathrm{~d},{ }^{3} J_{\mathrm{C}-\mathrm{F}}=9.3 \mathrm{~Hz}, 2 \times \mathrm{Ar}-\mathrm{C}\right), 128.5(2 \times$ $\mathrm{Ar}-\mathrm{C}), 128.5(\mathrm{Ar}-\mathrm{C}), 128.1(\mathrm{Ar}-\mathrm{C}), 116.9\left(\mathrm{~d},{ }^{2} \mathrm{~J}_{\mathrm{C}-\mathrm{F}}=22.7 \mathrm{~Hz}, 2 \times \mathrm{Ar}-\mathrm{C}\right), 68.4\left(\mathrm{OCH}_{2} \mathrm{Ph}\right), 51.1\left(\mathrm{OCH}_{3}\right)$. HRMS (ESITOF) $\mathrm{m} / \mathrm{z}(\mathrm{M}+\mathrm{Na})^{+}$calcd for $\mathrm{C}_{15} \mathrm{H}_{14} \mathrm{FNNaO} 3 \mathrm{~S} 330,0571$; found 330,0556 . 


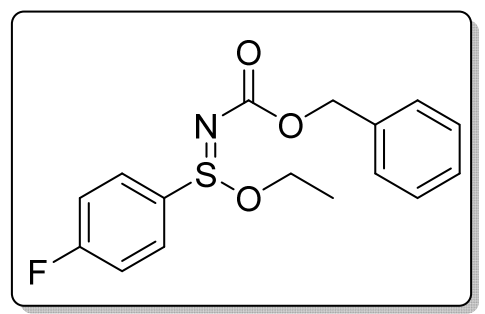

Prepared according to General Procedure B using sulfenamide 1d (106 $\mathrm{mg}$ ) in $\mathrm{EtOH}$, to afford sulfinimidate ester $\mathbf{3 k}$ as a waxy solid $(109 \mathrm{mg}$, $68 \%$ ). $R_{f} 0.6$ (40\% AcOEt in Hexane). IR (film) $/ \mathrm{cm}^{-1}$ 2982, 1649, 1589, $1490,1375,1226,1000,868 .{ }^{1} \mathrm{H}$ NMR $\left(500 \mathrm{MHz}, \mathrm{CDCl}_{3}, \mathrm{ppm}\right) \delta 7.95-$ $7.92(\mathrm{~m}, 2 \mathrm{H}, 2 \times \mathrm{Ar}-\mathrm{H}), 7.43(\mathrm{~d}, J=7.4 \mathrm{~Hz}, 2 \mathrm{H}, 2 \times \mathrm{Ar}-\mathrm{H}), 7.34(\mathrm{t}, J=$ $7.4 \mathrm{~Hz}, 2 \mathrm{H}, 2 \times \mathrm{Ar}-\mathrm{H}), 7.29(\mathrm{t}, J=7.4 \mathrm{~Hz}, 1 \mathrm{H}, \mathrm{Ar}-\mathrm{H}), 7.25-7.21(\mathrm{~m}, 2 \mathrm{H}$, $2 \times \mathrm{Ar}-\mathrm{H})$, ), 5.23 and $5.19(2 \times \mathrm{d}, J=12.3 \mathrm{~Hz}, \mathrm{AB}$ system, $2 \mathrm{H}, 2 \times$ $\mathrm{OCH} H \mathrm{Ph}), 4.21$ and $3.81\left(2 \times \mathrm{dq}, J=9.9,7.1 \mathrm{~Hz}, 2 \mathrm{H}, 2 \times \mathrm{SOCH} \mathrm{CH}_{3}\right)$, $1.26\left(\mathrm{t}, J=7.1 \mathrm{~Hz}, 3 \mathrm{H}, \mathrm{SOCH}_{2} \mathrm{CH}_{3}\right) .{ }^{19} \mathrm{~F} \mathrm{NMR}\left(470 \mathrm{MHz}, \mathrm{CDCl}_{3}\right) \delta-105.26\left(\mathrm{tt},{ }^{3} J_{\mathrm{F}-\mathrm{H}}=8.1,{ }^{4} J_{\mathrm{F}-\mathrm{H}}=5.0 \mathrm{~Hz}\right)$. ${ }^{13} \mathrm{C}$ NMR $\left(125 \mathrm{MHz}, \mathrm{CDCl}_{3}, \mathrm{ppm}\right) \delta 165.5\left(\mathrm{~d},{ }^{1} \mathrm{~J}_{\mathrm{C}-\mathrm{F}}=255.1 \mathrm{~Hz}, \mathrm{Ar}-\mathrm{C}_{\mathrm{q}}\right), 164.3(\mathrm{NCOO}), 136.8\left(\mathrm{Ar}-\mathrm{C}_{q}\right)$, $132.1\left(\mathrm{Ar}-\mathrm{C}_{\mathrm{q}}\right), 132.1\left(\mathrm{Ar}-\mathrm{C}_{\mathrm{q}}\right), 130.3\left(\mathrm{~d},{ }^{3} \mathrm{~J}_{\mathrm{C}-\mathrm{F}}=9.3 \mathrm{~Hz}, 2 \times \mathrm{Ar}-\mathrm{C}\right), 128.5(2 \times \mathrm{Ar}-\mathrm{C}), 128.4(2 \times \mathrm{Ar}-\mathrm{C}), 128.1$ $(\mathrm{Ar}-\mathrm{C}), 116.8\left(\mathrm{~d},{ }^{2} \mathrm{~J}_{\mathrm{C}-\mathrm{F}}=22.7 \mathrm{~Hz}, 2 \times \mathrm{Ar}-\mathrm{C}\right), 68.3\left(\mathrm{OCH}_{2} \mathrm{Ph}\right), 63.2\left(\mathrm{SOCH}_{2} \mathrm{CH}_{3}\right), 15.5\left(\mathrm{SOCH}_{2} \mathrm{CH}_{3}\right) . \mathrm{HRMS}$ (ESITOF) $\mathrm{m} / \mathrm{z}(\mathrm{M}+\mathrm{Na})^{+}$calcd for $\mathrm{C}_{16} \mathrm{H}_{16} \mathrm{FNNaO}_{3} \mathrm{~S} 344,0733$; found 344,0729.

\section{Methyl $\mathrm{N}-[$ (benzyloxy)carbonyl] -4-methylphenylsulfinimidate (3l)}

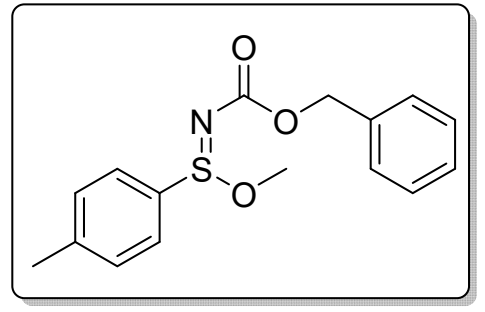

Prepared according to General Procedure B using sulfenamide 1e (104 $\mathrm{mg})$ in $\mathrm{MeOH}$, to afford sulfinimidate ester $3 \mathbf{l}$ as a waxy solid $(80 \mathrm{mg}$, $53 \%) . \mathrm{R}_{f} 0.45$ (30\% Hexane in $\left.\mathrm{Et}_{2} \mathrm{O}\right)$. IR (film)/ $\mathrm{cm}^{-1} 2941,1653,1450$, $1229,1084,951,812 .{ }^{1} \mathrm{H}$ NMR $\left(500 \mathrm{MHz}, \mathrm{CDCl}_{3}, \mathrm{ppm}\right) \delta 7.82-7.79(\mathrm{~m}$, $2 \mathrm{H}, 2 \times \mathrm{Ar}-\mathrm{H}), 7.45-7.42(\mathrm{~m}, 2 \mathrm{H}, 2 \times \mathrm{Ar}-\mathrm{H}), 7.36-7.32(\mathrm{~m}, 4 \mathrm{H}, 4 \times \mathrm{Ar}-$ $\mathrm{H}), 7.31-7.27(\mathrm{~m}, 1 \mathrm{H}, \mathrm{Ar}-\mathrm{H}), 5.24$ and $5.20\left(2 \times \mathrm{d},{ }^{2} \mathrm{~J}_{\mathrm{H}, \mathrm{H}}=12.3 \mathrm{~Hz}, \mathrm{AB}\right.$ system, $2 \mathrm{H}, 2 \times \mathrm{OCHHPh}), 3.49\left(\mathrm{~s}, 3 \mathrm{H}, \mathrm{SOCH}_{3}\right), 2.43\left(\mathrm{~s}, 3 \mathrm{H}, \mathrm{Ar}-\mathrm{CH}_{3}\right)$. ${ }^{13} \mathrm{C} \mathrm{NMR}\left(125 \mathrm{MHz}, \mathrm{CDCl}_{3}, \mathrm{ppm}\right) \delta 164.3(\mathrm{NCOO}), 144.1\left(\mathrm{Ar}-\mathrm{C}_{\mathrm{q}}\right), 136.8\left(\mathrm{Ar}-\mathrm{C}_{\mathrm{q}}\right), 131.9\left(\mathrm{Ar}-\mathrm{C}_{\mathrm{q}}\right), 130.2$ $(2 \times \mathrm{Ar}-\mathrm{C}), 128.5(2 \times \mathrm{Ar}-\mathrm{C}), 128.5(2 \times \mathrm{Ar}-\mathrm{C}), 128.0(\mathrm{Ar}-\mathrm{C}), 128.0(2 \times \mathrm{Ar}-\mathrm{C}), 68.2\left(\mathrm{OCH}_{2} \mathrm{Ph}\right), 50.7$ $\left(\mathrm{SOCH}_{3}\right), 21.7\left(\mathrm{Ar}-\mathrm{CH}_{3}\right)$. HRMS (ESITOF) $\mathrm{m} / \mathrm{z}(\mathrm{M}+\mathrm{Na})^{+}$calcd for $\mathrm{C}_{16} \mathrm{H}_{17} \mathrm{NNaO}_{3} \mathrm{~S}$ 326,0827; found 326,0825 .

\section{Ethyl $N-[($ benzyloxy)carbonyl]-4-methylphenylsulfinimidate $(3 \mathrm{~m})$}

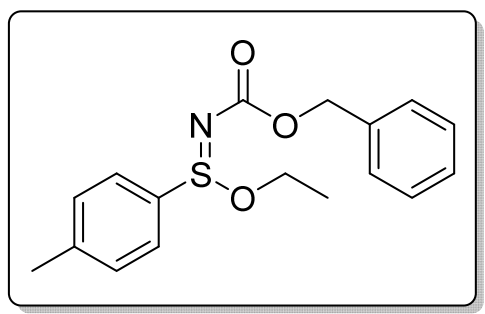

Prepared according to General Procedure B using sulfenamide 1e (104 $\mathrm{mg}$ ) in $\mathrm{EtOH}$, to afford sulfinimidate ester $3 \mathrm{~m}$ as a waxy solid $(82 \mathrm{mg}$, $52 \%)$. $\mathrm{R}_{f} 0.55$ (40\% AcOEt in Hexane). IR (film)/cm ${ }^{-1}$ 2982, 1653, 1450, 1230, 1085, 999, 867. ${ }^{1} \mathrm{H}$ NMR $\left(500 \mathrm{MHz}, \mathrm{CDCl}_{3}, \mathrm{ppm}\right) \delta 7.80(\mathrm{~d}, J=8.3$ $\mathrm{Hz}, 2 \mathrm{H}, 2 \times \mathrm{Ar}-\mathrm{H}), 7.43(\mathrm{~d}, J=7.1 \mathrm{~Hz}, 2 \mathrm{H}, 2 \times \mathrm{Ar}-\mathrm{H}), 7.37-7.33(\mathrm{~m}, 4 \mathrm{H}$, $4 \times \mathrm{Ar}-\mathrm{H}), 7.30-7.27(\mathrm{~m}, 1 \mathrm{H}, \mathrm{Ar}-\mathrm{H}), 5.23$ and $5.20(2 \times \mathrm{d}, J=12.3 \mathrm{~Hz}$, $\mathrm{AB}$ system, $2 \mathrm{H}, 2 \times \mathrm{OCH} H \mathrm{Ph}), 4.18$ and $3.77(2 \times \mathrm{dq}, J=9.9,7.1 \mathrm{~Hz}$, $\mathrm{ABX}$ system, $\left.2 \mathrm{H}, 2 \times \mathrm{OCH} \mathrm{CH}_{3}\right), 2.42\left(\mathrm{~s}, 3 \mathrm{H}, \mathrm{Ar}-\mathrm{CH}_{3}\right), 1.24(\mathrm{t}, J=7.1$ $\left.\mathrm{Hz}, 3 \mathrm{H}, \mathrm{OCH}_{2} \mathrm{CH}_{3}\right) .{ }^{13} \mathrm{C}$ NMR $\left(125 \mathrm{MHz}, \mathrm{CDCl}_{3}, \mathrm{ppm}\right) \delta 164.6$ (NCOO), $143.9\left(\mathrm{Ar}-\mathrm{C}_{\mathrm{q}}\right), 136.9\left(\mathrm{Ar}-\mathrm{C}_{\mathrm{q}}\right)$, $133.1\left(\mathrm{Ar}-\mathrm{C}_{\mathrm{q}}\right), 130.1(2 \times \mathrm{Ar}-\mathrm{C}), 128.5(2 \times \mathrm{Ar}-\mathrm{C}), 128.4(2 \times \mathrm{Ar}-\mathrm{C}), 128.0(\mathrm{Ar}-\mathrm{C}), 127.7(2 \times \mathrm{Ar}-\mathrm{C})$, $68.2\left(\mathrm{OCH}_{2} \mathrm{Ph}\right), 62.7\left(\mathrm{SOCH}_{2} \mathrm{CH}_{3}\right), 21.7\left(\mathrm{Ar}-\mathrm{CH}_{3}\right), 15.5\left(\mathrm{SOCH}_{2} \mathrm{CH}_{3}\right) . \mathrm{HRMS}(\mathrm{ESITOF}) \mathrm{m} / \mathrm{z}(\mathrm{M}+\mathrm{Na})^{+}$ calcd for $\mathrm{C}_{17} \mathrm{H}_{19} \mathrm{NNaO}_{3} \mathrm{~S} 340,0983$; found 340,0983. 


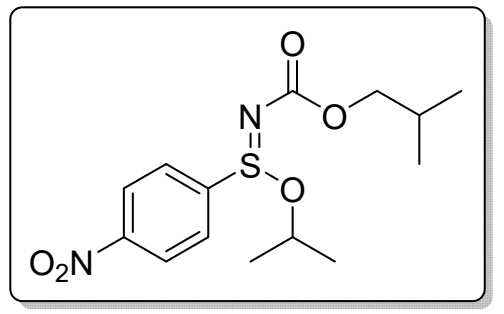

Prepared according to General Procedure B using sulfenamide 1f (119 $\mathrm{mg}$ ) in 2-propanol, to afford sulfinimidate ester $\mathbf{3 n}$ as a waxy solid without further purification (162 mg, 99\%). IR (film)/ $\mathrm{cm}^{-1} 3099,2963$, 1647, 1526, 1237, 971, 853, 729. ${ }^{1} \mathrm{H}$ NMR (500 MHz, $\left.\mathrm{CDCl}_{3}, \mathrm{ppm}\right) \delta$ 8.36-8.34 (m, 2H, $2 \times \mathrm{Ar}-\mathrm{H}), 8.10-8.08(\mathrm{~m}, 2 \mathrm{H}, 2 \times \mathrm{Ar}-\mathrm{H}), 4.84$ (hept, $\left.J=6.2 \mathrm{~Hz}, 1 \mathrm{H}, \mathrm{SOCH}\left(\mathrm{CH}_{3}\right)_{2}\right), 3.95$ and $3.90(2 \times \mathrm{dd}, J=10.5,6.7 \mathrm{~Hz}$, ABX system, $2 \mathrm{H}, 2 \times \mathrm{OCHHCH}\left(\mathrm{CH}_{3}\right)_{2}$ ), 1.99 (hept, $J=6.7 \mathrm{~Hz}, 1 \mathrm{H}$, $\left.\mathrm{OCH}_{2} \mathrm{CH}\left(\mathrm{CH}_{3}\right)_{2}\right), 1.42\left(\mathrm{~d}, J=6.2 \mathrm{~Hz}, 3 \mathrm{H}, \mathrm{OCHCH}_{3}\right), 1.27\left(\mathrm{~d}, J=6.2 \mathrm{~Hz}, 3 \mathrm{H}, \mathrm{OCHCH}_{3}\right), 0.94(\mathrm{~d}, J=6.7$ $\left.\mathrm{Hz}, 6 \mathrm{H}, 2 \times \mathrm{OCH}_{2} \mathrm{CHCH}_{3}\right){ }^{13} \mathrm{C} \mathrm{NMR}\left(125 \mathrm{MHz}, \mathrm{CDCl}_{3}\right) \delta 164.7(\mathrm{NCOO}), 150.2\left(\mathrm{Ar}-\mathrm{C}_{\mathrm{q}}\right), 144.1\left(\mathrm{Ar}-\mathrm{C}_{\mathrm{q}}\right)$, $128.9(2 \times \mathrm{Ar}-\mathrm{C}), 124.3(2 \times \mathrm{Ar}-\mathrm{C}), 76.7\left(\mathrm{SOCH}\left(\mathrm{CH}_{3}\right)_{2}\right), 73.0\left(\mathrm{OCH}_{2} \mathrm{CH}_{2}\right), 28.0\left(\mathrm{OCH}_{2} \mathrm{CH}\left(\mathrm{CH}_{3}\right)_{2}\right), 23.6$ $(\mathrm{OCHCH} 3), 23.5(\mathrm{OCHCH} 3), 19.2\left(2 \times \mathrm{OCH}_{2} \mathrm{CHCH}_{3}\right)$. HRMS (ESITOF) m/z $(\mathrm{M}+\mathrm{Na})^{+}$calcd for $\mathrm{C}_{14} \mathrm{H}_{20} \mathrm{~N}_{2} \mathrm{NaO}_{5} \mathrm{~S} 351,0991$; found 351,0988.

\section{Ethyl $N$-[(isobutyloxy)carbonyl]-4-trifluoromethylphenylsulfinimidate (30)}

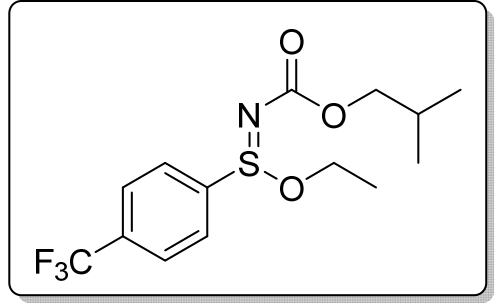

Prepared according to General Procedure B using sulfenamide 1g (131 $\mathrm{mg})$ in $\mathrm{EtOH}$, to afford sulfinimidate ester 30 as a waxy solid $(123 \mathrm{mg}$, $73 \%) . R_{f} 0.6$ (30\% AcOEt in Hexane). IR (film)/cm ${ }^{-1}$ 2959, 1729, 1642, 1321, 1242, 1060, 840. ${ }^{1} \mathrm{H}$ NMR $\left(500 \mathrm{MHz}, \mathrm{CDCl}_{3}, \mathrm{ppm}\right) \delta 8.06(\mathrm{~d}, \mathrm{~J}=$ $8.3 \mathrm{~Hz}, 2 \mathrm{H}, 2 \times \mathrm{Ar}-\mathrm{H}), 7.80(\mathrm{~d}, J=8.3 \mathrm{~Hz}, 2 \mathrm{H}, 2 \times \mathrm{Ar}-\mathrm{H}), 4.27$ and 3.87 $\left(2 \times \mathrm{dq}, J=10.0,7.1 \mathrm{~Hz}, \mathrm{ABX}\right.$ system, $\left.2 \mathrm{H}, 2 \times \mathrm{SOCH} \mathrm{HCH}_{3}\right), 3.97$ and $3.94\left(2 \times \mathrm{dd}, J=10.5,6.8 \mathrm{~Hz}, \mathrm{ABX}\right.$ system, $\left.2 \mathrm{H}, 2 \times \mathrm{OCHHCH}\left(\mathrm{CH}_{3}\right)_{2}\right)$, 2.02 (hept, $\left.J=6.7 \mathrm{~Hz}, 1 \mathrm{H}, \mathrm{CH}_{2} \mathrm{CH}\left(\mathrm{CH}_{3}\right)_{2}\right), 1.30$ (t, $J=7.1 \mathrm{~Hz}, 3 \mathrm{H}$ $\left.\mathrm{SOCH}_{2} \mathrm{CH}_{3}\right), 0.97\left(\mathrm{~d}, J=6.7 \mathrm{~Hz}, 6 \mathrm{H}, 2 \times \mathrm{OCH}_{2} \mathrm{CHCH}_{3}\right) .{ }^{19} \mathrm{~F} \mathrm{NMR}\left(470 \mathrm{MHz}, \mathrm{CDCl}_{3}\right) \delta-63.1 .{ }^{13} \mathrm{C} \mathrm{NMR}$ $\left(125 \mathrm{MHz}, \mathrm{CDCl}_{3}\right) \delta 164.6(\mathrm{NCOO}), 140.7\left(\mathrm{Ar}-\mathrm{C}_{\mathrm{q}}\right), 134.7\left(\mathrm{q},{ }^{2} \mathrm{~J}_{\mathrm{C}-\mathrm{F}}=33.0 \mathrm{~Hz}, \mathrm{Ar}-\mathrm{C}_{\mathrm{q}}\right), 128.4(2 \times \mathrm{Ar}-\mathrm{C})$, $126.5\left(\mathrm{q},{ }^{3} J_{\mathrm{C}-\mathrm{F}}=3.7 \mathrm{~Hz}, 2 \times \mathrm{Ar}-\mathrm{C}\right), 123.4\left(\mathrm{q},{ }^{1} \mathrm{~J}_{\mathrm{C}-\mathrm{F}}=273.0 \mathrm{~Hz}, \mathrm{Ar}-\mathrm{CF}_{3}\right), 73.0\left(\mathrm{OCH}_{2} \mathrm{CH}\left(\mathrm{CH}_{3}\right)_{2}\right), 64.0$ $\left(\mathrm{SOCH}_{2} \mathrm{CH}_{3}\right), 28.1\left(\mathrm{OCH}_{2} \mathrm{CH}\left(\mathrm{CH}_{3}\right)_{2}\right), 19.3\left(2 \times \mathrm{OCH}_{2} \mathrm{CHCH}_{3}\right), 15.6\left(\mathrm{SOCH}_{2} \mathrm{CH}_{3}\right) . \mathrm{HRMS}$ (ESITOF) m/z $(\mathrm{M}+\mathrm{Na})^{+}$calcd for $\mathrm{C}_{14} \mathrm{H}_{18} \mathrm{~F}_{3} \mathrm{NNaO}_{3} \mathrm{~S} 360,0857$; found 360,0852 .

\section{Methyl $N-[($ isobutyloxy)carbonyl]-3-methoxyphenylsulfinimidate (3p)}

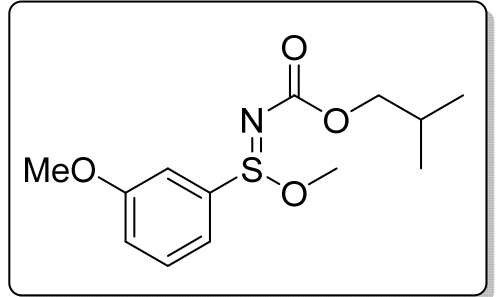

Prepared according to General Procedure C using sulfenamide 1h (105 $\mathrm{mg}$ ) in $\mathrm{MeOH}$, to afford sulfinimidate ester $3 p$ as a waxy solid without further purification (128 mg, 90\%). IR (film)/ $\mathrm{cm}^{-1}$ 2959, 2841, 1652, 1594, 1231, 1035, 956, 786. ${ }^{1} \mathrm{H}$ NMR $\left(300 \mathrm{MHz}, \mathrm{CDCl}_{3}, \mathrm{ppm}\right) \delta 7.48-$ $7.45(\mathrm{~m}, 3 \mathrm{H}, 3 \times \mathrm{Ar}-\mathrm{H}), 7.14-7.10(\mathrm{~m}, 1 \mathrm{H}, \mathrm{Ar}-\mathrm{H}), 3.96(\mathrm{~d}, J=6.8 \mathrm{~Hz}$, $\left.2 \mathrm{H}, \mathrm{OCH}_{2} \mathrm{CH}\left(\mathrm{CH}_{3}\right)_{2}\right), 3.88\left(\mathrm{~s}, 3 \mathrm{H}, \mathrm{OCH}_{3}\right), 3.54\left(\mathrm{~s}, 3 \mathrm{H}, \mathrm{SOCH}_{3}\right) 2.03$ (hept, $\left.J=6.7 \mathrm{~Hz}, 1 \mathrm{H}, \mathrm{OCH}_{2} \mathrm{CH}\left(\mathrm{CH}_{3}\right)_{2}\right), 0.98(\mathrm{~d}, J=6.7 \mathrm{~Hz}, 6 \mathrm{H}, 2 \times$ $\left.\mathrm{OCH}_{2} \mathrm{CHCH}_{3}\right) \cdot{ }^{13} \mathrm{C} \mathrm{NMR}\left(75 \mathrm{MHz}, \mathrm{CDCl}_{3}\right) \delta 164.5$ (NCOO), $160.5\left(\mathrm{Ar}-\mathrm{C}_{\mathrm{q}}\right), 136.8\left(\mathrm{Ar}-\mathrm{C}_{\mathrm{q}}\right), 130.5(\mathrm{Ar}-\mathrm{C})$, $120.2(\mathrm{Ar}-\mathrm{C}), 119.5(\mathrm{Ar}-\mathrm{C}), 112.4(2 \times \mathrm{Ar}-\mathrm{C}), 72.9\left(\mathrm{OCH}_{2} \mathrm{CH}\right), 55.9\left(\mathrm{OCH}_{3}\right), 51.2\left(\mathrm{SOCH}_{3}\right)$, 28.2 $\left(\mathrm{OCH}_{2} \mathrm{CH}\left(\mathrm{CH}_{3}\right)_{2}\right), 19.4\left(2 \times \mathrm{OCH}_{2} \mathrm{CHCH}_{3}\right)$. HRMS (ESITOF) $\mathrm{m} / \mathrm{z}(\mathrm{M}+\mathrm{Na})^{+}$calcd for $\mathrm{C}_{13} \mathrm{H}_{19} \mathrm{NNaO}_{4} \mathrm{~S}$ 308,0932 ; found 308,0932 .

\section{Ethyl $N-[($ isobutyloxy)carbonyl]-2-naphthylsulfinimidate (3q)}

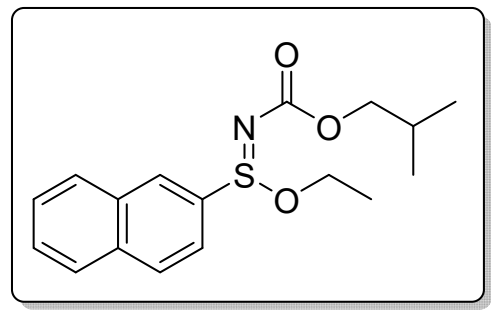

Prepared according to General Procedure B using sulfenamide 1i (123 $\mathrm{mg}$ ) in $\mathrm{EtOH}$, to afford sulfinimidate ester $\mathbf{3 q}$ as a waxy solid without further purification (142 mg, 89\%). IR (film)/ $/ \mathrm{cm}^{-1} 3056,2959,1646,1373$, 1233, 999, 866, 748. ${ }^{1} \mathrm{H}$ NMR $\left(500 \mathrm{MHz}, \mathrm{CDCl}_{3}, \mathrm{ppm}\right) \delta 8.53(\mathrm{~s}, 1 \mathrm{H}, \mathrm{Ar}-$ $\mathrm{H}), 8.01-8.00(\mathrm{~m}, 2 \mathrm{H}, 2 \times \mathrm{Ar}-\mathrm{H}), 7.93-7.88(\mathrm{~m}, 2 \mathrm{H}, 2 \times \mathrm{Ar}-\mathrm{H}), 7.66-$ $7.60(\mathrm{~m}, 2 \mathrm{H}, 2 \times \mathrm{Ar}-\mathrm{H}), 4.26$ and $3.81(\mathrm{dq}, J=10.0,7.1 \mathrm{~Hz}, \mathrm{ABX}$ system, $\left.2 \mathrm{H}, 2 \times \mathrm{SOCH} \mathrm{HCH}_{3}\right), 4.01$ and $3.99(2 \times \mathrm{dd}, J=11.9,6.0 \mathrm{~Hz}, \mathrm{ABX}$ system, $\left.2 \mathrm{H}, 2 \times \mathrm{OCHHCH}\left(\mathrm{CH}_{3}\right)_{2}\right), 2.06$ (hept, $\left.J=6.8 \mathrm{~Hz}, 1 \mathrm{H}, \mathrm{OCH}_{2} \mathrm{CH}\left(\mathrm{CH}_{3}\right)_{2}\right), 1.28(\mathrm{t}, J=7.1 \mathrm{~Hz}, 3 \mathrm{H}$, 
$\left.\mathrm{SOCH}_{2}, \mathrm{CH}_{3}\right), 1.00\left(\mathrm{~d}, J=6.8 \mathrm{~Hz}, 6 \mathrm{H}, 2 \times \mathrm{SOCHCH}_{3}\right) .{ }^{13} \mathrm{C} \mathrm{NMR}\left(125 \mathrm{MHz}, \mathrm{CDCl}_{3}\right) \delta 164.8(\mathrm{NCOO})$, $135.2\left(\mathrm{Ar}-\mathrm{C}_{\mathrm{q}}\right), 133.3\left(\mathrm{Ar}-\mathrm{C}_{\mathrm{q}}\right), 132.7\left(\mathrm{Ar}-\mathrm{C}_{\mathrm{q}}\right), 129.8(\mathrm{Ar}-\mathrm{C}), 129.3(\mathrm{Ar}-\mathrm{C}), 129.1(\mathrm{Ar}-\mathrm{C}), 128.9(\mathrm{Ar}-\mathrm{C})$, 128.1 (Ar-C), $127.7(\mathrm{Ar}-\mathrm{C}), 122.9(\mathrm{Ar}-\mathrm{C}), 72.9\left(\mathrm{OCH}_{2} \mathrm{CH}\right), 62.8\left(\mathrm{SOCH}_{2} \mathrm{CH}_{3}\right) 28.2\left(\mathrm{OCH}_{2} \mathrm{CH}\left(\mathrm{CH}_{3}\right)_{2}\right)$, $19.4\left(2 \times \mathrm{OCH}_{2} \mathrm{CHCH}_{3}\right), 15.6\left(\mathrm{SOCH}_{2} \mathrm{CH}_{3}\right)$. HRMS (ESITOF) $\mathrm{m} / \mathrm{z}(\mathrm{M}+\mathrm{Na})^{+}$calcd for $\mathrm{C}_{17} \mathrm{H}_{21} \mathrm{NNaO}_{3} \mathrm{~S}$ 342,1140 ; found 342,1140 .

\section{Isopropyl $\mathbf{N}-[$ (isobutyloxy)carbonyl]cyclohexylsulfinimidate (3r)}

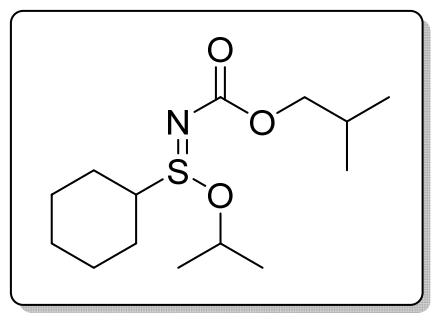

Prepared according to General Procedure C using sulfenamide $1 \mathbf{j}$ (101 mg) in 2-propanol, to afford sulfinimidate ester $3 \mathrm{r}$ as a waxy solid without further purification (142 mg, 89\%). IR (film)/ $\mathrm{cm}^{-1} 2957,2929,1730,1644,1374$, 1239, 907, 831. ${ }^{1} \mathrm{H}$ NMR $\left(500 \mathrm{MHz}, \mathrm{CDCl}_{3}\right.$, ppm) $\delta 4.71$ (hept, $1 \mathrm{H}, J=6.2$ $\left.\mathrm{Hz}, \mathrm{OCH}\left(\mathrm{CH}_{3}\right)_{2}\right), 3.90$ and $3.85(2 \times \mathrm{dd}, 2 \mathrm{H}, \mathrm{ABX}$ system, $J=10.4,6.9 \mathrm{~Hz}$, $\left.2 \times \mathrm{OCH} H \mathrm{CH}\left(\mathrm{CH}_{3}\right)_{2}\right), 2.99\left(\mathrm{t}, 1 \mathrm{H}, J=11.1 \mathrm{~Hz}, \mathrm{SCH}\left(\mathrm{CH}_{2}\right)_{2}\right), 2.17-2.10(\mathrm{~m}$, $\left.2 \mathrm{H}, \mathrm{CH}_{2}\right), 1.98\left(\mathrm{~m}, 1 \mathrm{H}, J=6.8 \mathrm{~Hz}, \mathrm{OCH}_{2} \mathrm{CH}\left(\mathrm{CH}_{3}\right)_{2}\right), 1.89-1.83\left(\mathrm{~m}, 2 \mathrm{H}, \mathrm{CH}_{2}\right)$, 1.70-1.67 (m, 1H, CH), 1.65-1.55 (m, 1H, CH), 1.52-1.29 (m, 4H, $\left.2 \times \mathrm{CH}_{2},\right), 1.34$ and $1.32(\mathrm{~d}, 3 \mathrm{H}, J=$ $\left.6.2 \mathrm{~Hz}, \mathrm{OCHCH}_{3}\right), 0.94\left(\mathrm{~d}, 6 \mathrm{H}, J=7.6 \mathrm{~Hz}, \mathrm{CH}\left(\mathrm{CH}_{3}\right)_{2}\right) .{ }^{13} \mathrm{C} \mathrm{NMR}\left(75 \mathrm{MHz}, \mathrm{CDCl}_{3}\right) \delta 166.4$ (NCOO), 77.4 $\left(\mathrm{SOCH}_{2} \mathrm{CH}_{2}\right), 72.5\left(\mathrm{NCOOCH}_{2} \mathrm{CH}\right), 59.7\left(\mathrm{SCH}\left(\mathrm{CH}_{2}\right)\right), 28.2\left(\mathrm{NCOOCH}_{2} \mathrm{CH}\right), 26.2\left(2 \times \mathrm{CH}_{2}\right), 25.5\left(\mathrm{CH}_{2}\right)$, $25.3\left(2 \times \mathrm{CH}_{2}\right), 23.5\left(\mathrm{CH}_{3}\right), 23.2\left(\mathrm{CH}_{3}\right), 19.4\left(2 \times \mathrm{CH}_{3}\right)$. HRMS (ESITOF) m/z $(\mathrm{M}+\mathrm{Na})+$ calcd for $\mathrm{C}_{14} \mathrm{H}_{27} \mathrm{NNaO}_{3} \mathrm{~S} 312,1609$; found 312,1598.

\section{Butyl N-[(isobutyloxy)carbonyl]cyclohexylsulfinimidate (3s)}

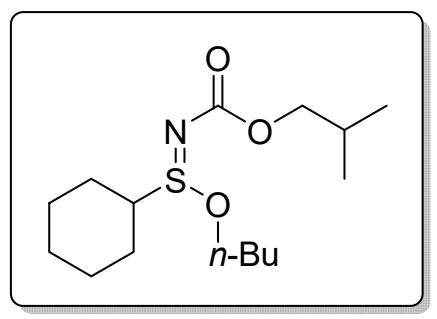

Prepared according to General Procedure B using sulfenamide $\mathbf{1 j}$ (101 mg) in 1-butanol, to afford sulfinimidate ester $3 \mathrm{~s}$ as a waxy solid $(118 \mathrm{mg}, 78 \%)$. $\mathrm{R}_{f} 0.45$ (50\% AcOEt in Hexane). IR (film) $/ \mathrm{cm}^{-1}$ 2955, 2857, 1644, 1452, 1237, 916, 750. ${ }^{1} \mathrm{H}$ NMR (500 MHz, $\left.\mathrm{CDCl}_{3}, \mathrm{ppm}\right) \delta 4.13(\mathrm{~m}, 2 \mathrm{H}, 2 \times$ $\left.\mathrm{OCH} H C H_{2}\right), 3.89$ and $3.84(2 \times \mathrm{dd}, 2 \mathrm{H}, \mathrm{ABX}$ system, $J=10.0,7.1 \mathrm{~Hz}, 2 \times$ $\left.\mathrm{OCH} H \mathrm{CH}\left(\mathrm{CH}_{3}\right)_{2}\right), 3.01\left(\mathrm{tt}, 1 \mathrm{H}, J=10.0,7.1 \mathrm{~Hz}, \mathrm{SCH}\left(\mathrm{CH}_{2}\right)_{2}\right), 2.14-2.08(\mathrm{~m}$, $\left.2 \mathrm{H}, \mathrm{CH}_{2}\right), 2.04-1.91(\mathrm{~m}, 1 \mathrm{H}, \mathrm{CH}), 1.88-1.83\left(\mathrm{~m}, 2 \mathrm{H}, \mathrm{CH}_{2}\right), 1.69-1.62(\mathrm{~m}$, $2 \mathrm{H}), 1.54-1.24(\mathrm{~m}, 8 \mathrm{H}), 0.93\left(\mathrm{~d}, 6 \mathrm{H}, J=7.6 \mathrm{~Hz}, \mathrm{CH}\left(\mathrm{CH}_{3}\right)_{2}\right), 0.91(\mathrm{t}, 3 \mathrm{H}, J=$ $\left.7.4 \mathrm{~Hz}, \mathrm{CH}_{2} \mathrm{CH}_{3}\right) \cdot{ }^{13} \mathrm{C} \mathrm{NMR}\left(125 \mathrm{MHz}, \mathrm{CDCl}_{3}\right) \delta 166.2(\mathrm{NCOO}), 72.5\left(\mathrm{SOCH}_{2} \mathrm{CH}_{2}\right), 71.2\left(\mathrm{NCOOCH}_{2} \mathrm{CH}\right)$, $59.5\left(\mathrm{SCH}\left(\mathrm{CH}_{2}\right)\right), 32.2\left(\mathrm{CH}_{2}\right), 28.1\left(\mathrm{NCOOCH}_{2} \mathrm{CH}\right), 26.5\left(\mathrm{CH}_{2}\right), 26.1\left(\mathrm{CH}_{2}\right), 25.5\left(\mathrm{CH}_{2}\right), 25.2\left(2 \times \mathrm{CH}_{2}\right)$, $19.4\left(2 \times \mathrm{CH}_{3}\right), 19.0\left(\mathrm{CH}_{2}\right), 13.7\left(\mathrm{CH}_{3}\right)$. HRMS (ESITOF) $\mathrm{m} / \mathrm{z}(\mathrm{M}+\mathrm{Na})^{+}$calcd for $\mathrm{C}_{15} \mathrm{H}_{29} \mathrm{NNaO}_{3} \mathrm{~S} 326,1766$; found 326,1759 . 


\section{DoE optimization study for $4 a$ synthesis}

Optimization study: Full factorial $2^{2}$ screening design for the preparation of benzyl(phenyl(piperidin-1-yl)- $\lambda^{4}$-sulfanylidene)carbamate $4 a$ in toluene.

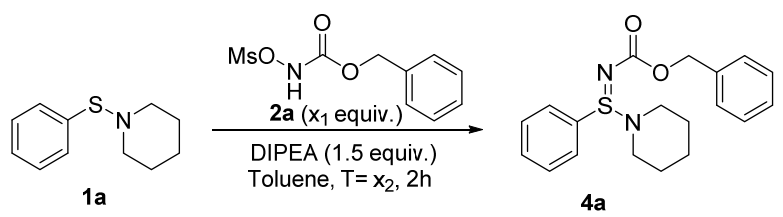

Table 1. Screening Design

\begin{tabular}{|c|c|c|c|c|c|}
\hline Entry & $\begin{array}{c}X_{1} \text { coded } \\
\text { 2a (Equiv.) }\end{array}$ & $\begin{array}{c}\mathrm{X}_{2} \text { coded } \\
\text { (Temperature) }\end{array}$ & $\begin{array}{c}\mathrm{X}_{1} \text { uncoded } \\
\text { 2a (Equiv.) }\end{array}$ & $\begin{array}{c}\mathrm{X}_{2} \text { uncoded } \\
\text { (Temperature) }\end{array}$ & Yield of $4 a^{(a)}$ \\
\hline 1 & -1 & -1 & 1.6 & $0^{\circ} \mathrm{C}$ & $45 \%$ \\
\hline 2 & +1 & -1 & 1.9 & $0^{\circ} \mathrm{C}$ & $53 \%$ \\
\hline 3 & -1 & +1 & 1.6 & $25^{\circ} \mathrm{C}$ & $73 \%$ \\
\hline 4 & +1 & +1 & 1.9 & $25^{\circ} \mathrm{C}$ & $95 \%$ \\
\hline
\end{tabular}

(a) Reaction yields were calculated through the 1H NMR analysis of the reaction crude with the use of an internal standard.

Choice of levels and coding

$\mathrm{x}_{1}$ High $=1.9 ; \mathrm{x}_{1}$ Low $=1.6$

$x_{2}$ High $=298 \mathrm{~K} ; x_{2}$ Low $=273 \mathrm{~K}$

$\mathrm{M}=($ High + Low $) / 2 ; \mathrm{M}_{1}=1.75 ; \mathrm{M}_{2}=283$

$\mathrm{H}=(\mathrm{High}-$ Low $) / 2 ; \mathrm{H}_{1}=0.15 ; \mathrm{H}_{2}=10$

Coded $=($ Uncoded $-\mathrm{M}) / \mathrm{H}$

The experimental responses were fitted with a first degree interaction model $y=\beta_{0}+\beta_{1} x_{1}+\beta_{2} x_{2}+$ $\beta_{12} x_{1} x_{2}$ giving the estimated response surface equation:

$$
\text { Yield } 4 a=66,5+7,5 x_{1}+17,5 x_{2}+3,5 x_{1} x_{2}
$$

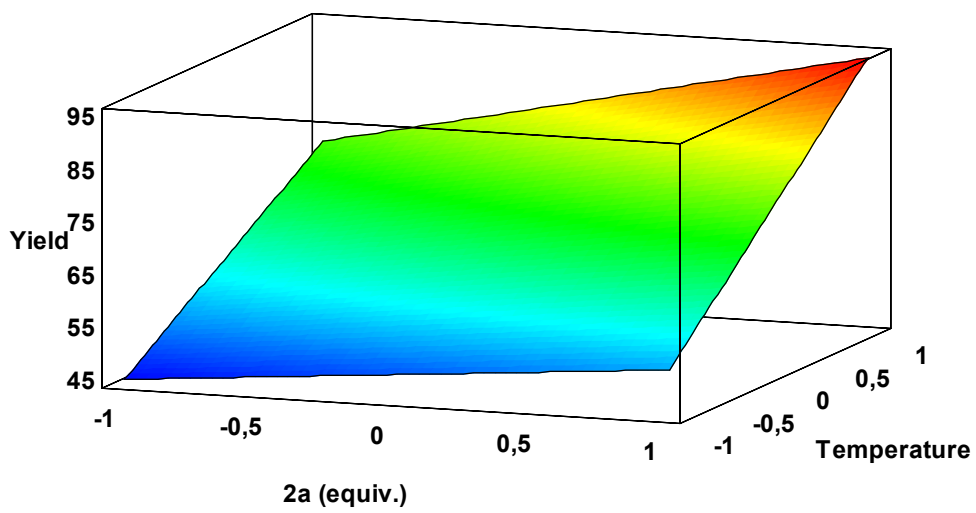


Optimization study: Full factorial $2^{2}$ screening design and Steepest Ascent approach for the preparation of benzyl-(phenyl(piperidin-1-yl)- $\lambda^{4}$-sulfanylidene)carbamate $4 a$ in $\mathrm{CH}_{2} \mathrm{Cl}_{2}$.

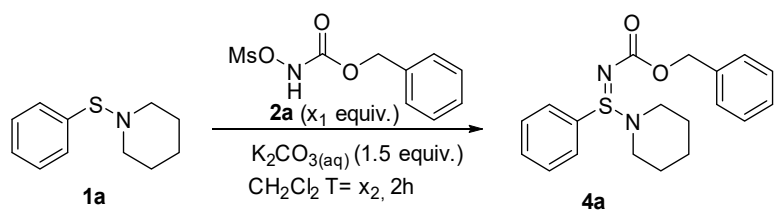

Table 1. Screening Design

\begin{tabular}{|c|c|c|c|c|c|}
\hline Entry & $\begin{array}{c}\mathrm{X}_{1} \text { coded } \\
\text { 2a (Equiv.) }\end{array}$ & $\begin{array}{c}\mathbf{X}_{2} \text { coded } \\
\text { (Temperature) }\end{array}$ & $\begin{array}{c}X_{1} \text { uncoded } \\
\text { 2a (Equiv.) }\end{array}$ & $\begin{array}{c}\mathbf{X}_{2} \text { uncoded } \\
\text { (Temperature) }\end{array}$ & Yield of $4 a^{(a)}$ \\
\hline 1 & -1 & -1 & 1.3 & $0^{\circ} \mathrm{C}$ & $29 \%$ \\
\hline 2 & +1 & -1 & 1.6 & $0^{\circ} \mathrm{C}$ & $50 \%$ \\
\hline 3 & -1 & +1 & 1.3 & $25^{\circ} \mathrm{C}$ & $43 \%$ \\
\hline 4 & +1 & +1 & 1.6 & $25^{\circ} \mathrm{C}$ & $68 \%$ \\
\hline
\end{tabular}

(a) Reaction yields were calculated through the $1 \mathrm{H}$ NMR analysis of the reaction crude with the use of an internal standard.

\section{Choice of levels and coding}

$\mathrm{x}_{1}$ High $=1.6 ; \mathrm{x}_{1}$ Low $=1.3$

$x_{2}$ High $=298 \mathrm{~K} ; \mathrm{x}_{2}$ Low $=273 \mathrm{~K}$

$\mathrm{M}=($ High + Low $) / 2 ; \mathrm{M}_{1}=1.45 ; \mathrm{M}_{2}=283$

$\mathrm{H}=(\mathrm{High}-$ Low $) / 2 ; \mathrm{H}_{1}=0.15 ; \mathrm{H}_{2}=10$

Coded $=($ Uncoded $-\mathrm{M}) / \mathrm{H}$

The experimental responses were fitted with a first degree interaction model $y=\beta_{0}+\beta_{1} x_{1}+\beta_{2} x_{2}+$ $\beta_{12} x_{1} x_{2}$ giving the estimated response surface equation:

$$
\text { Yield } 4 a=47,5+11,5 x_{1}+8,0 x_{2}+1,0 x_{1} x_{2}
$$

\section{Response Surface}

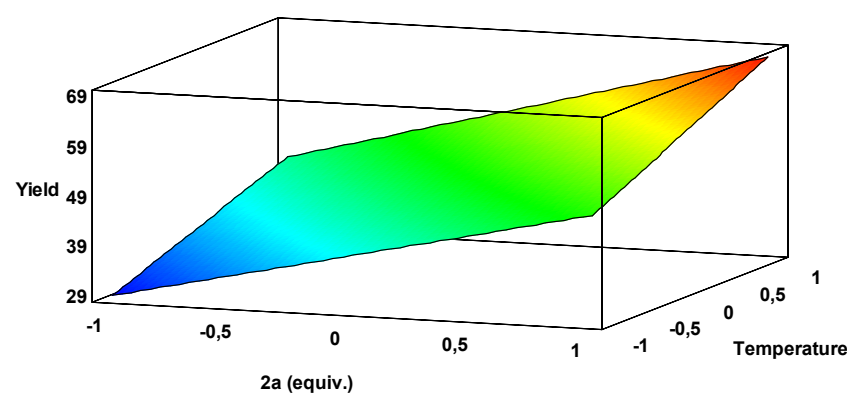

\section{Steepest Ascent Approach}

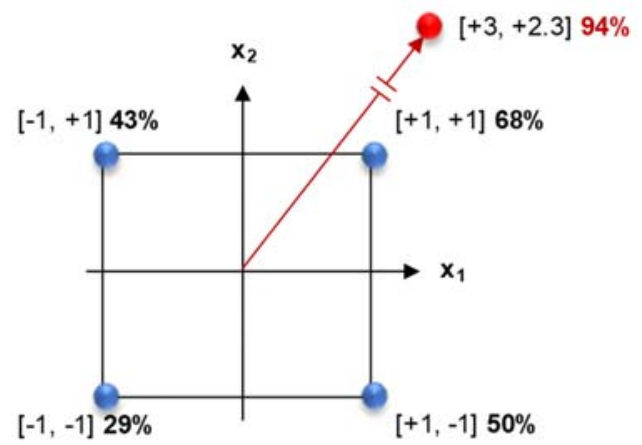

The reaction conditions were further optimized following the direction of Steepest Ascent, allowing the preparation of $4 \mathbf{a}$ in $94 \%$ of yield $\left(1.9\right.$ equiv. of $\left.2 \mathbf{a}, 33^{\circ} \mathrm{C}\right)$. 


\section{Sulfinamidines}

\subsection{General Procedure D for sulfinamidines synthesis}

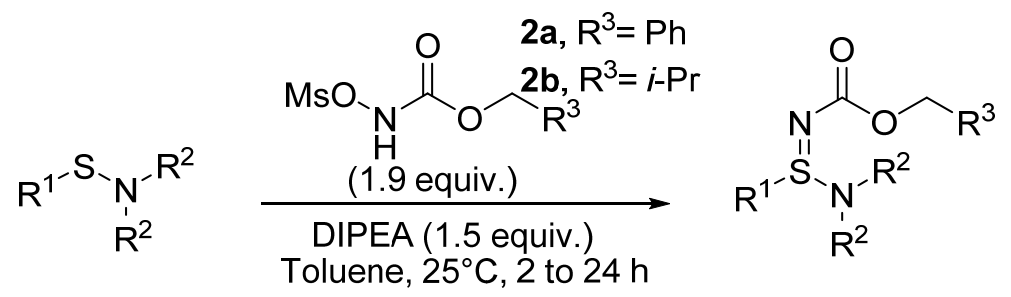

To a stirred solution of sulfenamide $(0.5 \mathrm{mmol}, 1.0$ equiv. $)$ in toluene $(5 \mathrm{~mL}, 0.1 \mathrm{M})$, alkyl((methylsulfonyl)oxy)carbamate (0.95 mmol, 1.9 equiv.) and diisopropylethylamine (DIPEA, $0.75 \mathrm{mmol}, 1.5$ equiv.) were added in one portion, and the reaction mixture was stirred at $25^{\circ} \mathrm{C}$ for 2 to $24 \mathrm{~h}$, as indicated for each entry. Subsequently, $\mathrm{H}_{2} \mathrm{O}(15 \mathrm{~mL})$ was added and the aqueous mixture was extracted with AcOEt $(3 \times 15 \mathrm{~mL})$. The organic layers were collected, dried $\left(\mathrm{Na}_{2} \mathrm{SO}_{4}\right)$, filtered and the solvent removed under reduced pressure. Purification by flash chromatography on silica gel afforded the corresponding sulfinamidine.

\subsection{Procedure $E$ for the synthesis of sulfinamidine $4 a$ on $2 \mathrm{mmol}$ scale}

To a stirred solution of sulfenamide $1 \mathrm{a}(386 \mathrm{mg}, 2.0 \mathrm{mmol}, 1.0$ equiv.) in toluene (20 mL, $0.1 \mathrm{M})$, benzyl((methylsulfonyl)oxy)carbamate 2a (932 mg, $3.8 \mathrm{mmol}, 1.9$ equiv.) and diisopropylethylamine (DIPEA, $388 \mathrm{mg}, 3,0 \mathrm{mmol}, 1.5$ equiv.) were added in one portion, and the reaction mixture was stirred at $25^{\circ} \mathrm{C}$ for $2 \mathrm{~h}$. Subsequently, $\mathrm{H}_{2} \mathrm{O}(50 \mathrm{~mL})$ was added and the aqueous mixture was extracted with AcOEt $(3 \times$ $50 \mathrm{~mL})$. The organic layers were collected, dried $\left(\mathrm{Na}_{2} \mathrm{SO}_{4}\right)$, filtered and the solvent removed under reduced pressure. Purification by flash chromatography on silica gel afforded sulfinamidine $\mathbf{4 a}$ in $95 \%$ of yield. 


\subsection{Characterization data for sulfinamidines $4 a-0$}

\section{Benzyl [phenyl(piperidin-1-yl)- $\lambda^{4}$-sulfanylidene]carbamate (4a)}

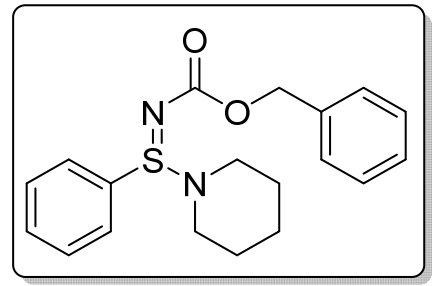

Prepared according to Procedure E using sulfenamide 1a (386 mg) to afford sulfinamidine $4 \mathrm{a}$ as a white solid $(650 \mathrm{mg}, 95 \%)$. $\mathrm{R}_{f} 0.2$ (30\% AcOEt in Hexane). IR (film) $/ \mathrm{cm}^{-1} 2939,2851,1730,1642,1444,1243,1064,862,752 .{ }^{1} \mathrm{H}$ NMR $\left(500 \mathrm{MHz}, \mathrm{CDCl}_{3}, \mathrm{ppm}\right) \delta 7.88-7.86(\mathrm{~m}, 2 \mathrm{H}, \mathrm{Ar}-\mathrm{H}), 7.51-7.49(\mathrm{~m}, 3 \mathrm{H}$, $\mathrm{Ar}-\mathrm{H}), 7.44_{5}(\mathrm{~d}, J=7.4 \mathrm{~Hz}, 2 \mathrm{H}, \mathrm{Ar}-\mathrm{H}), 7.33(\mathrm{t}, J=7.4 \mathrm{~Hz}, 2 \mathrm{H}, \mathrm{Ar}-\mathrm{H}), 7.28$ $(\mathrm{d}, J=7.2 \mathrm{~Hz}, 1 \mathrm{H}, \mathrm{Ar}-\mathrm{H}), 5.225$ and $5.16(2 \times \mathrm{d}, J=12.4 \mathrm{~Hz}, \mathrm{AB}$ system, $2 \mathrm{H}, 2 \times \mathrm{OCH} H \mathrm{Ph}), 3.20-3.16(\mathrm{~m}, 2 \mathrm{H}, 2 \times \mathrm{CH}), 2.96-2.92(\mathrm{~m}, 2 \mathrm{H}, 2 \times \mathrm{CH})$, 1.64-1.47 (m, 6H, 6xCH). ${ }^{13} \mathrm{C}$ NMR (125 MHz, CDCl $) \delta 165.3$ (NCOO), $137.7\left(\mathrm{Ar}-\mathrm{C}_{\mathrm{q}}\right), 134.6\left(\mathrm{Ar}-\mathrm{C}_{\mathrm{q}}\right)$, $131.6(\mathrm{Ar}-\mathrm{C}), 129.2(\mathrm{Ar}-\mathrm{C}), 128.4(\mathrm{Ar}-\mathrm{C}), 128.3(\mathrm{Ar}-\mathrm{C}), 128.2(\mathrm{Ar}-\mathrm{C}), 127.8(\mathrm{Ar}-\mathrm{C}), 67.7\left(\mathrm{OCH}_{2} \mathrm{Ph}\right)$, $48.3\left(\mathrm{NCH}_{2}\right), 26.2\left(\mathrm{NCH}_{2} \mathrm{CH}_{2}\right), 23.7\left(\mathrm{NCH}_{2} \mathrm{CH}_{2} \mathrm{CH}_{2}\right)$. HRMS (ESITOF) $\mathrm{m} / \mathrm{z}(\mathrm{M}+\mathrm{H})^{+}$calcd for $\mathrm{C}_{19} \mathrm{H}_{23} \mathrm{~N}_{2} \mathrm{O}_{2} \mathrm{~S}$ 343,1480 ; found 343,1483 .

\section{Isobutyl [phenyl(piperidin-1-yl)- $\lambda^{4}$-sulfanylidene]carbamate (4b)}

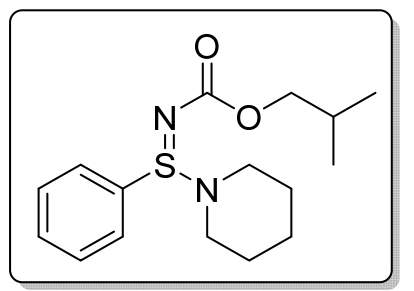

Prepared according to General Procedure $\mathrm{D}$ with a reaction time of $2 \mathrm{~h}$, using sulfenamide $1 \mathrm{a}(97 \mathrm{mg})$ to afford sulfinamidine $\mathbf{4 b}$ as a waxy solid $(138 \mathrm{mg}$, $90 \%) . \mathrm{R}_{f} 0.2$ (30\% Hexane in $\left.\mathrm{Et}_{2} \mathrm{O}\right)$. IR (film)/ $/ \mathrm{cm}^{-1} 2937,2854,1633,1471$, 1235, 974, 859, 753. ${ }^{1} \mathrm{H}$ NMR $\left(500 \mathrm{MHz}, \mathrm{CDCl}_{3}, \mathrm{ppm}\right) \delta$ 7.89-7.86 (m, 2H, 2 $\times \mathrm{Ar}-\mathrm{H}), 7.51-7.50(\mathrm{~m}, 3 \mathrm{H}, 3 \times \mathrm{Ar}-\mathrm{H}), 3.94-3.88(\mathrm{~m}, \mathrm{ABX}$ system, $2 \mathrm{H}, 2 \times$ $\mathrm{OCH} H \mathrm{CH}), 3.21-3.16\left(\mathrm{~m}, 2 \mathrm{H}, 2 \times \mathrm{NCHHCH}_{2}\right), 2.97-2.93(\mathrm{~m}, 2 \mathrm{H}, 2 \times$ $\mathrm{NCH} \mathrm{HCH}_{2}$ ), 2.02 (hept, $\left.J=6.7 \mathrm{~Hz}, 1 \mathrm{H}, \mathrm{OCH}_{2} \mathrm{CH}\left(\mathrm{CH}_{3}\right)_{2}\right), 1.66-1.49(\mathrm{~m}, 6 \mathrm{H}, 6$ $\times \mathrm{CH}), 0.97\left(\mathrm{~d}, \mathrm{~J}=6.7 \mathrm{~Hz}, 6 \mathrm{H}, 2 \times \mathrm{OCH}_{2} \mathrm{CHCH}_{3}\right) \cdot{ }^{13} \mathrm{C} \mathrm{NMR}\left(125 \mathrm{MHz}, \mathrm{CDCl}_{3}\right) \delta 165.8(\mathrm{NCOO}), 134.7$ $\left(\mathrm{Ar}-\mathrm{C}_{\mathrm{q}}\right), 131.5(\mathrm{Ar}-\mathrm{C}), 129.2(2 \times \mathrm{Ar}-\mathrm{C}), 128.2(2 \times \mathrm{Ar}-\mathrm{C}), 72.3\left(\mathrm{OCH}_{2} \mathrm{CH}\right), 48.3\left(2 \times \mathrm{NCH}_{2} \mathrm{CH}_{2}\right), 28.3$ $\left(\mathrm{OCH}_{2} \mathrm{CH}\left(\mathrm{CH}_{3}\right)_{2}\right), 26.3\left(2 \times \mathrm{NCH}_{2} \mathrm{CH}_{2} \mathrm{CH}_{2}\right) 23.7\left(\mathrm{NCH}_{2} \mathrm{CH}_{2} \mathrm{CH}_{2}\right), 19.5\left(2 \times \mathrm{OCH}_{2} \mathrm{CHCH}_{3}\right) . \mathrm{HRMS}$ (ESITOF) $\mathrm{m} / \mathrm{z}(\mathrm{M}+\mathrm{Na})^{+}$calcd for $\mathrm{C}_{16} \mathrm{H}_{24} \mathrm{~N}_{2} \mathrm{NaO}_{2} \mathrm{~S} 331,1456$; found 331,1453 .

\section{Benzyl [(4-methoxyphenyl)(piperidin-1-yl)- $\lambda^{4}$-sulfanylidene]carbamate (4c)}

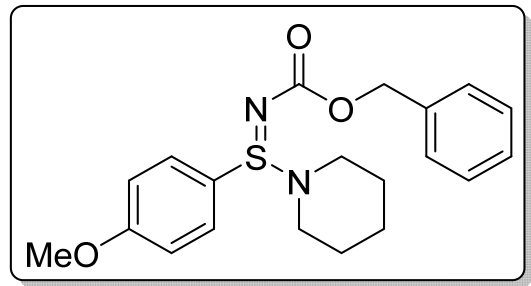

Prepared according to General Procedure $D$ with a reaction time of 2 $\mathrm{h}$, using sulfenamide $\mathbf{1 b}(112 \mathrm{mg})$ to afford sulfinamidine $\mathbf{4 c}$ as a waxy solid (144 mg, 77\%). $\mathrm{R}_{f} 0.2$ (30\% AcOEt in Hexane). IR (film) $/ \mathrm{cm}^{-1}$ 2939, 2851, 1644, 1593, 1494, 1244, 1086, 861. ${ }^{1} \mathrm{H}$ NMR $(500 \mathrm{MHz}$, $\left.\mathrm{CDCl}_{3}, \mathrm{ppm}\right) \delta 7.77(\mathrm{~d}, J=8.9 \mathrm{~Hz}, 2 \mathrm{H}, \mathrm{Ar}-\mathrm{H}), 7.44(\mathrm{~d}, J=7.5 \mathrm{~Hz}, 2 \mathrm{H}$, $\mathrm{Ar}-\mathrm{H}$ ), $7.33\left(\mathrm{t}, J=7.5 \mathrm{~Hz}, 2 \mathrm{H}, \mathrm{Ar}-\mathrm{H}\right.$ ), 7.28 (d overlapping $\mathrm{CDCl}_{3}, J=$ $8.9 \mathrm{~Hz}, 1 \mathrm{H}, \mathrm{Ar}-\mathrm{H}), 6.99(\mathrm{~d}, \mathrm{~J}=8.9 \mathrm{~Hz}, 2 \mathrm{H}, \mathrm{Ar}-\mathrm{H}), 5.21_{5}$ and $5.14_{5}(2$ $\times \mathrm{d}, J=12.4 \mathrm{~Hz}, \mathrm{AB}$ system, $2 \mathrm{H}, 2 \times \mathrm{OCH} H \mathrm{Ph}), 3.85\left(\mathrm{~s}, 3 \mathrm{H}, \mathrm{OCH}_{3}\right), 3.17-3.12\left(\mathrm{~m}, 2 \mathrm{H}, 2 \times \mathrm{NCHHCH}_{2}\right)$, 2.94-2.90 (m, 2H, $\left.2 \times \mathrm{NCH} \mathrm{CCH}_{2}\right), 1.63-146(\mathrm{~m}, 6 \mathrm{H}, 6 \times \mathrm{CH}) .{ }^{13} \mathrm{C} \mathrm{NMR}\left(125 \mathrm{MHz}, \mathrm{CDCl}_{3}\right) \delta 165.3$ (NCOO), $162.3\left(\mathrm{Ar}-\mathrm{C}_{\mathrm{q}}\right), 137.7\left(\mathrm{Ar}-\mathrm{C}_{\mathrm{q}}\right), 129.9(\mathrm{Ar}-\mathrm{C}), 128.4(\mathrm{Ar}-\mathrm{C}), 128.3(\mathrm{Ar}-\mathrm{C}), 127.7(\mathrm{Ar}-\mathrm{C}), 125.2$ $\left(\mathrm{Ar}-\mathrm{C}_{\mathrm{q}}\right), 114.7(\mathrm{Ar}-\mathrm{C}), 67.6\left(\mathrm{OCH}_{2} \mathrm{Ph}\right), 55.7\left(\mathrm{OCH}_{3}\right), 48.0\left(2 \times \mathrm{NCH}_{2} \mathrm{CH}_{2}\right), 26.2\left(2 \times \mathrm{NCH}_{2} \mathrm{CH}_{2}\right), 23.8$ $\left(\mathrm{NCH}_{2} \mathrm{CH}_{2} \mathrm{CH}_{2}\right.$ ). HRMS (ESITOF) m/z (M+Na) ${ }^{+}$calcd for $\mathrm{C}_{20} \mathrm{H}_{24} \mathrm{~N}_{2} \mathrm{NaO}_{3} \mathrm{~S} 395,1405$; found 395, 1400 .

\section{Benzyl [(4-chlorophenyl)(piperidin-1-yl)- $\lambda^{4}$-sulfanylidene]carbamate (4d)}

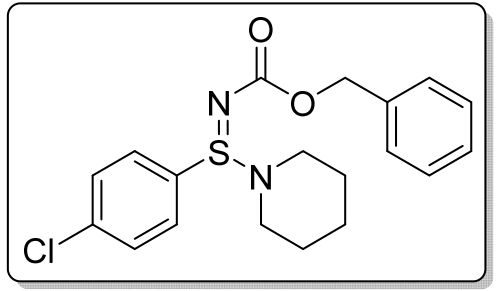

Prepared according to General Procedure $D$ with a reaction time of $2 \mathrm{~h}$, using sulfenamide $1 \mathrm{c}(114 \mathrm{mg})$ to afford sulfinamidine $\mathbf{4 d}$ as a waxy solid (111 mg, 60\%). $\mathrm{R}_{f} 0.3$ (30\% Hexane in $\left.\mathrm{Et}_{2} \mathrm{O}\right)$. IR (film) $/ \mathrm{cm}^{-1} 2938$, 2853, 1732, 1644, 1375, 1258, 1081, 799. ${ }^{1} \mathrm{H}$ NMR $\left(500 \mathrm{MHz}, \mathrm{CDCl}_{3}\right.$, ppm) $\delta 7.81(\mathrm{~d}, J=8.7 \mathrm{~Hz}, 2 \mathrm{H}, 2 \times \operatorname{Ar}-H), 7.47(\mathrm{~d}, J=8.7 \mathrm{~Hz}, 2 \mathrm{H}, 2 \times$ $\operatorname{Ar}-H), 7.43(\mathrm{~d}, J=7.4 \mathrm{~Hz}, 2 \mathrm{H}, 2 \times \mathrm{Ar}-H), 7.34(\mathrm{t}, J=7.4 \mathrm{~Hz}, 2 \mathrm{H}, 2 \times$ $\operatorname{Ar}-H), 7.29(\mathrm{t}, J=7.4 \mathrm{~Hz}, 1 \mathrm{H}, \mathrm{Ar}-H), 5.21$ and $5.15(2 \times \mathrm{d}, J=12.3 \mathrm{~Hz}$, AB system, 2H, $2 \times \mathrm{OCH} H \mathrm{Ph}), 3.19-3.14\left(\mathrm{~m}, 2 \mathrm{H}, 2 \times \mathrm{NCHHCH}_{2}\right), 2.95-2.90\left(\mathrm{~m}, 2 \mathrm{H}, 2 \times \mathrm{NCH} \mathrm{CH}_{2}\right)$, 
1.63-1.49 (m, 6H, $6 \times \mathrm{CH}) .{ }^{13} \mathrm{C}$ NMR $\left(125 \mathrm{MHz}, \mathrm{CDCl}_{3}\right) \delta 165.2(\mathrm{NCOO}), 138.2\left(\mathrm{Ar}-\mathrm{C}_{\mathrm{q}}\right), 137.5\left(\mathrm{Ar}-\mathrm{C}_{\mathrm{q}}\right)$, $133.2\left(\mathrm{Ar}-\mathrm{C}_{\mathrm{q}}\right), 129.7(2 \times \mathrm{Ar}-\mathrm{C}), 129.5(2 \times \mathrm{Ar}-\mathrm{C}), 128.4(2 \times \mathrm{Ar}-\mathrm{C}), 128.3(2 \times \mathrm{Ar}-\mathrm{C}), 127.9(\mathrm{Ar}-\mathrm{C})$, $67.8\left(\mathrm{OCH}_{2} \mathrm{Ph}\right), 48.4\left(2 \times \mathrm{NCH}_{2} \mathrm{CH}_{2}\right), 26.2\left(2 \times \mathrm{NCH}_{2} \mathrm{CH}_{2}\right) 23.6\left(\mathrm{NCH}_{2} \mathrm{CH}_{2} \mathrm{CH}_{2}\right) . \mathrm{HRMS}(\mathrm{ESITOF}) \mathrm{m} / \mathrm{z}$ $(\mathrm{M}+\mathrm{Na})^{+}$calcd for $\mathrm{C}_{19} \mathrm{H}_{21} \mathrm{ClN}_{2} \mathrm{NaO}_{2} \mathrm{~S} 399,0910$; found 399,0924.

\section{Benzyl [(4-fluorophenyl)(piperidin-1-yl)- $\lambda^{4}$-sulfanylidene]carbamate (4e)}

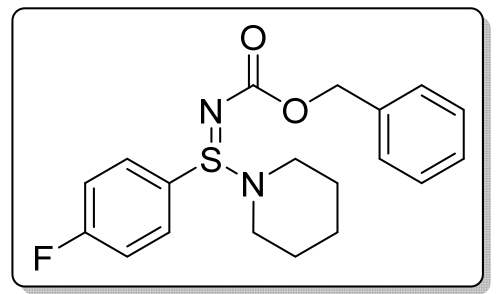

Prepared according to General Procedure D with a reaction time of $2 \mathrm{~h}$, using sulfenamide $1 \mathrm{~d}(106 \mathrm{mg})$ to afford sulfinamidine $4 \mathbf{e}$ as a waxy solid (90 mg, 50\%). R 0.45 (40\% AcOEt in Hexane). IR (film)/cm ${ }^{-1} 2944$, 2854, 1732, 1639, 1264, 864, 738. ${ }^{1} \mathrm{H}$ NMR $\left(300 \mathrm{MHz}, \mathrm{CDCl}_{3}, \mathrm{ppm}\right) \delta$ 7.88-7.85 (m, 2H, Ar-H), $7.43(\mathrm{~d}, J=7.3 \mathrm{~Hz}, 2 \mathrm{H}, \mathrm{Ar}-\mathrm{H}), 7.36-7.28(\mathrm{~m}$, $3 \mathrm{H}, \mathrm{Ar}-\mathrm{H}), 7.19(\mathrm{t}, J=8.6 \mathrm{~Hz}, 1 \mathrm{H}, \mathrm{Ar}-\mathrm{H}), 5.22$ and $5.14(2 \times \mathrm{d}, J=12.4$ $\mathrm{Hz}$, AB system, $2 \mathrm{H}, 2 \times \mathrm{OCH} H \mathrm{Ph}), 3.19-3.14\left(\mathrm{~m}, 2 \mathrm{H}, 2 \times \mathrm{NCHHCH}_{2}\right)$, 2.95-2.90 (m, 2H, $\left.2 \times \mathrm{NCHHCH}_{2}\right), 1.63-1.50(\mathrm{~m}, 6 \mathrm{H}, 6 \times \mathrm{CH}) .{ }^{19} \mathrm{~F} \mathrm{NMR}\left(282 \mathrm{MHz}, \mathrm{CDCl}_{3}\right) \delta-108.15(\mathrm{tt}$, $\left.{ }^{3} J_{F-H}=8.1,{ }^{4} J_{F-H}=5.0 \mathrm{~Hz}\right) .{ }^{13} \mathrm{C} \mathrm{NMR}\left(125 \mathrm{MHz}, \mathrm{CDCl}_{3}\right) \delta 165.2(\mathrm{NCOO}), 164.8\left(\mathrm{~d},{ }^{1} \mathrm{~J}_{\mathrm{C}-\mathrm{F}}=253.3 \mathrm{~Hz}, \mathrm{Ar}-\right.$ $\left.\mathrm{C}_{\mathrm{q}}\right), 137.5\left(\mathrm{Ar}-\mathrm{C}_{\mathrm{q}}\right), 130.6\left(\mathrm{~d},{ }^{3} \mathrm{~J}_{\mathrm{C}-\mathrm{F}}=9.0 \mathrm{~Hz}, \mathrm{Ar}-\mathrm{C}\right), 128.7\left(\mathrm{Ar}-\mathrm{C}_{\mathrm{q}}\right), 128.4(\mathrm{Ar}-\mathrm{C}), 128.3\left(\mathrm{Ar}-\mathrm{C}_{\mathrm{q}}\right), 128.3(\mathrm{Ar}-$ C), $127.8(\mathrm{Ar}-\mathrm{C}), 116.5\left(\mathrm{~d},{ }^{2} \mathrm{~J}_{\mathrm{C}-\mathrm{F}}=22.5 \mathrm{~Hz}, \mathrm{Ar}-\mathrm{C}\right), 67.8\left(\mathrm{OCH}_{2} \mathrm{Ph}\right), 48.2\left(2 \times \mathrm{NCH}_{2} \mathrm{CH}_{2}\right), 26.2(2 \times$ $\left.\mathrm{NCH}_{2} \mathrm{CH}_{2}\right)$, $23.6\left(\mathrm{NCH}_{2} \mathrm{CH}_{2} \mathrm{CH}_{2}\right)$. HRMS (ESITOF) $\mathrm{m} / \mathrm{z}(\mathrm{M}+\mathrm{Na})^{+}$calcd for $\mathrm{C}_{19} \mathrm{H}_{21} \mathrm{FN}_{2} \mathrm{NaO}_{2} \mathrm{~S} 383,1205$; found 383,1212 .

\section{Benzyl [piperidin-1-yl(p-tolyl)- $\lambda^{4}$-sulfanylidene]carbamate (4f)}

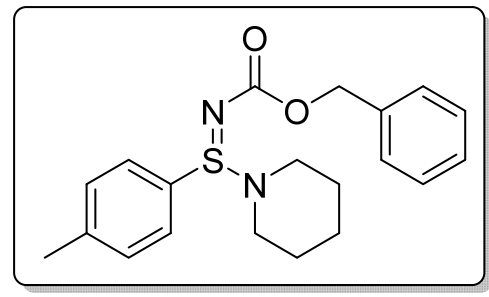

Prepared according to General Procedure D with a reaction time of $2 \mathrm{~h}$, using sulfenamide $1 \mathrm{e}(104 \mathrm{mg})$ to afford sulfinamidine $\mathbf{4 f}$ as a waxy solid (112 mg, 63\%). $\mathrm{R}_{f} 0.45$ (30\% AcOEt in Hexane). IR (film)/ $\mathrm{cm}^{-1} 2942$, 2855, 1731, 1633, 1377, 1252, 908, 732. ${ }^{1} \mathrm{H}$ NMR $\left(500 \mathrm{MHz}, \mathrm{CDCl}_{3}\right.$, ppm) $\delta 7.73(\mathrm{~d}, J=8.4 \mathrm{~Hz}, 2 \mathrm{H}, 2 \times \mathrm{Ar}-\mathrm{H}), 7.44-7.43(\mathrm{~m}, 2 \mathrm{H}, 2 \times \mathrm{Ar}-\mathrm{H})$, 7.37-7.27 (m, 5H, $5 \times \mathrm{Ar}-\mathrm{H}), 5.21$ and $5.15(2 \times \mathrm{d}, J=12.4 \mathrm{~Hz}, \mathrm{AB}$ system, $2 \mathrm{H}, 2 \times \mathrm{OCH} H \mathrm{Ph}), 3.18-3.13\left(\mathrm{~m}, 2 \mathrm{H}, 2 \times \mathrm{NCHHCH}_{2}\right), 2.94-$ $2.90\left(\mathrm{~m}, 2 \mathrm{H}, 2 \times \mathrm{NCHHCH}_{2}\right), 2.40\left(\mathrm{~s}, 3 \mathrm{H}, \mathrm{Ar}-\mathrm{CH}_{3}\right), 1.62-1.47(\mathrm{~m}, 6 \mathrm{H}, 6 \times \mathrm{CH}) \cdot{ }^{13} \mathrm{C} \mathrm{NMR}(125 \mathrm{MHz}$, $\left.\mathrm{CDCl}_{3}\right) \delta 165.3(\mathrm{NCOO}), 142.2\left(\mathrm{Ar}-\mathrm{C}_{\mathrm{q}}\right), 137.7\left(\mathrm{Ar}-\mathrm{C}_{\mathrm{q}}\right), 131.2\left(\mathrm{Ar}-\mathrm{C}_{\mathrm{q}}\right), 129.9(2 \times \mathrm{Ar}-\mathrm{C}), 128.4(2 \times \mathrm{Ar}-$ C), $128.3(2 \times \mathrm{Ar}-\mathrm{C}), 128.1(2 \times \mathrm{Ar}-\mathrm{C}), 127.7(\mathrm{Ar}-\mathrm{C}), 67.6\left(\mathrm{OCH}_{2} \mathrm{Ph}\right), 48.2\left(2 \times \mathrm{NCH}_{2} \mathrm{CH}_{2}\right), 26.2(2 \times$ $\left.\mathrm{NCH}_{2} \mathrm{CH}_{2}\right), 23.7\left(\mathrm{NCH}_{2} \mathrm{CH}_{2} \mathrm{CH}_{2}\right), 21.4\left(\mathrm{Ar}-\mathrm{CH}_{3}\right)$. HRMS (ESITOF) $\mathrm{m} / \mathrm{z}(\mathrm{M}+\mathrm{Na})^{+}$calcd for $\mathrm{C}_{20} \mathrm{H}_{24} \mathrm{~N}_{2} \mathrm{NaO}_{2} \mathrm{~S}$ 379,1456 ; found 379,1455 .

\section{Benzyl [4-nitrophenyl(piperidin-1-yl)- $\lambda^{4}$-sulfanylidene]carbamate (4g)}

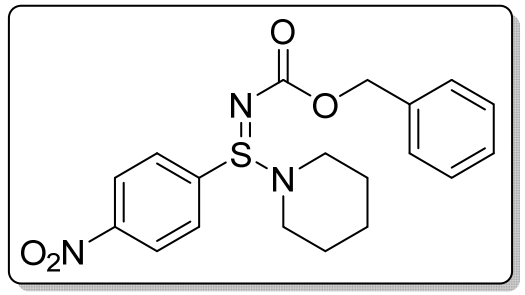

Prepared according to General Procedure D with a reaction time of 24 $\mathrm{h}$, using sulfenamide $\mathbf{1 f}(119 \mathrm{mg})$ to afford sulfinamidine $\mathbf{4 g}$ as a waxy solid (89 mg, 46\%). $\mathrm{R}_{f} 0.25$ (40\% AcOEt in Hexane). IR (film)/ $\mathrm{cm}^{-1}$ 3096, 2936, 1638, 1449, 1237, 855. ${ }^{1} \mathrm{H}$ NMR $\left(500 \mathrm{MHz}, \mathrm{CDCl}_{3}, \mathrm{ppm}\right)$ $\delta 8.34(\mathrm{~d}, J=9.2 \mathrm{~Hz}, 2 \mathrm{H}, 2 \times \mathrm{Ar}-H), 8.10(\mathrm{~d}, J=9.2 \mathrm{~Hz}, 2 \mathrm{H}, 2 \times \mathrm{Ar}-$ $H), 7.44(\mathrm{~d}, J=7.3 \mathrm{~Hz}, 2 \mathrm{H}, 2 \times \mathrm{Ar}-H), 7.35(\mathrm{t}, J=7.3 \mathrm{~Hz}, 2 \mathrm{H}, 2 \times \mathrm{Ar}-$ $H), 7.30(\mathrm{t}, J=7.3 \mathrm{~Hz}, 1 \mathrm{H}, \mathrm{Ar}-H), 5.23$ and $5.17(2 \times \mathrm{d}, J=12.3 \mathrm{~Hz}$, $2 \mathrm{H}, \mathrm{AB}$ system, OCHHPh), 3.25-3.20 (m, 2H, $\left.2 \times \mathrm{NCHHCH}_{2}\right), 2.97-2.93\left(\mathrm{~m}, 2 \mathrm{H}, 2 \times \mathrm{NCH}^{2} \mathrm{CH}_{2}\right), 1.69-$ $1.59(\mathrm{~m}, 6 \mathrm{H}, 6 \times \mathrm{CH}) .{ }^{13} \mathrm{C}$ NMR $\left(125 \mathrm{MHz}, \mathrm{CDCl}_{3}\right) \delta 165.0(\mathrm{NCOO}), 150.0\left(\mathrm{Ar}-\mathrm{C}_{\mathrm{q}}\right), 141.9\left(\mathrm{Ar}-\mathrm{C}_{\mathrm{q}}\right), 137.3$ $\left(\mathrm{Ar}-\mathrm{C}_{\mathrm{q}}\right), 129.7(2 \times \mathrm{Ar}-\mathrm{C}), 128.5(2 \times \mathrm{Ar}-\mathrm{C}), 128.4(2 \times \mathrm{Ar}-\mathrm{C}), 128.0(\mathrm{Ar}-\mathrm{C}), 124.2$ (2 $\times$ Ar-C), 68.1 $\left(\mathrm{OCH}_{2} \mathrm{Ph}\right), 48.8\left(2 \times \mathrm{NCH}_{2} \mathrm{CH}_{2}\right), 26.2\left(2 \times \mathrm{NCH}_{2} \mathrm{CH}_{2}\right) 23.4\left(\mathrm{NCH}_{2} \mathrm{CH}_{2} \mathrm{CH}_{2}\right) . \mathrm{HRMS}(\mathrm{ESITOF}) \mathrm{m} / \mathrm{z}(\mathrm{M}+\mathrm{Na})^{+}$ calcd for $\mathrm{C}_{19} \mathrm{H}_{21} \mathrm{~N}_{3} \mathrm{NaO}_{4} \mathrm{~S} 410,1150$; found 410,1137 . 


\section{Benzyl [4-trifluoromethylphenyl(piperidin-1-yl)- $\lambda^{4}$-sulfanylidene]carbamate (4h)}

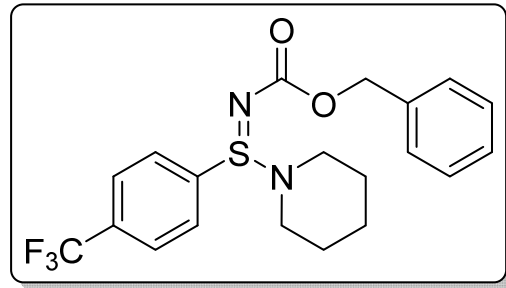

$\left.2 \mathrm{H}, 2 \times \mathrm{SNCH} \mathrm{HCH}_{2}\right), 2.97-2.92\left(\mathrm{~m}, 2 \mathrm{H}, 2 \times \mathrm{SNCHHCH}_{2}\right), 1.66-1.59(\mathrm{~m}, 6 \mathrm{H}, 6 \times \mathrm{CH}) .{ }^{19} \mathrm{~F} \mathrm{NMR}(282$ $\left.\mathrm{MHz}, \mathrm{CDCl}_{3}\right) \delta-62.9 .{ }^{13} \mathrm{C} \mathrm{NMR}\left(125 \mathrm{MHz}, \mathrm{CDCl}_{3}, \mathrm{ppm}\right) \delta 165.2$ (NCOO), $139.0\left(\mathrm{Ar}-\mathrm{C}_{\mathrm{q}}\right), 133.7$ (q, ${ }^{2} \mathrm{~J}_{\mathrm{C}-\mathrm{F}}$ $\left.=32.8 \mathrm{~Hz}, \mathrm{Ar}-\mathrm{C}_{\mathrm{q}}\right), 128.9(\mathrm{Ar}-\mathrm{C}), 128.5(\mathrm{Ar}-\mathrm{C}), 128.4(\mathrm{Ar}-\mathrm{C}), 127.9(\mathrm{Ar}-\mathrm{C}), 126.2\left(\mathrm{q},{ }^{3} \mathrm{~J}_{\mathrm{C}-\mathrm{F}}=3.6 \mathrm{~Hz}, \mathrm{Ar}-\right.$ C), $123.56\left(\mathrm{~d},{ }^{1} \mathrm{~J}_{\mathrm{C}-\mathrm{F}}=272.8 \mathrm{~Hz}, \mathrm{Ar}-\mathrm{CF}_{3}\right), 68.0\left(\mathrm{COOCH}_{2}\right), 48.6\left(2 \times \mathrm{NCH}_{2} \mathrm{CH}_{2}\right), 26.3\left(2 \times \mathrm{NCH}_{2} \mathrm{CH}_{2}\right)$, $23.5\left(\mathrm{NCH}_{2} \mathrm{CH}_{2} \mathrm{CH}_{2}\right)$. HRMS (ESITOF) $\mathrm{m} / \mathrm{z}(\mathrm{M}+\mathrm{Na})^{+}$calcd for $\mathrm{C}_{20} \mathrm{H}_{21} \mathrm{~F}_{3} \mathrm{~N}_{2} \mathrm{NaO}_{2} \mathrm{~S}$ 433,1174; found $433,1171$.

\section{Benzyl [(3-methoxyphenyl)(pyrrolidin-1-yl)- $\lambda^{4}$-sulfanylidene]carbamate (4i)}

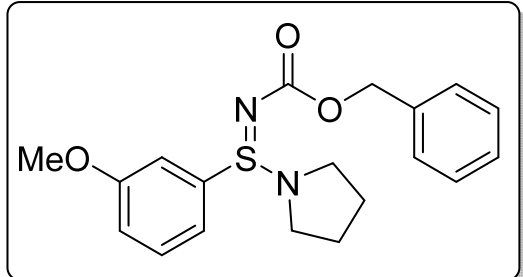

Prepared according to General Procedure D with a reaction time of 2 $\mathrm{h}$, using sulfenamide $\mathbf{1 h}(104 \mathrm{mg})$ to afford sulfinamidine $4 \mathrm{i}$ as a waxy solid (140 mg, 78\%). $\mathrm{R}_{f} 0.25$ (30\% Hexane in $\left.\mathrm{Et}_{2} \mathrm{O}\right)$. IR (film)/cm ${ }^{-1}$ 2961, 2875, 1725, 1629, 1232, 1035, 857, 749. ${ }^{1} \mathrm{H}$ NMR $(500 \mathrm{MHz}$, $\left.\mathrm{CDCl}_{3}, \mathrm{ppm}\right) \delta 7.44-7.42(\mathrm{~m}, 4 \mathrm{H}, \mathrm{Ar}-\mathrm{H}), 7.39(7, \mathrm{~J}=8.0 \mathrm{~Hz}, 1 \mathrm{H}, \mathrm{Ar}-$ $\mathrm{H}), 7.33(\mathrm{t}, J=7.5 \mathrm{~Hz}, 2 \mathrm{H}, \mathrm{Ar}-\mathrm{H}), 7.28(\mathrm{~d}, J=7.5 \mathrm{~Hz}, 1 \mathrm{H}, \mathrm{Ar}-\mathrm{H}) 7.00$ $(\mathrm{dd}, J=8.0,2.5 \mathrm{~Hz}, 1 \mathrm{H}, \mathrm{Ar}-\mathrm{H}), 5.21$ and $5.15(2 \times \mathrm{d}, J=12.4 \mathrm{~Hz}, \mathrm{AB}$ system, $2 \mathrm{H}, 2 \times \mathrm{OCH} H \mathrm{Ph}), 3.84\left(\mathrm{~s}, 3 \mathrm{H}, \mathrm{OCH}_{3}\right), 3.43-3.38\left(\mathrm{~m}, 2 \mathrm{H}, 2 \times \mathrm{NCHHCH}_{2}\right), 3.16-3.12(\mathrm{~m}, 2 \mathrm{H}, 2$ $\left.\times \mathrm{NCHHCH}_{2}\right), 1.88-1.82-1.79(\mathrm{~m}, 4 \mathrm{H}, 4 \times \mathrm{CH}) .{ }^{13} \mathrm{C} \mathrm{NMR}\left(125 \mathrm{MHz}, \mathrm{CDCl}_{3}\right) \delta 165.5(\mathrm{NCOO}), 160.3(\mathrm{Ar}-$ $\left.\mathrm{C}_{\mathrm{q}}\right), 137.3\left(\mathrm{Ar}-\mathrm{C}_{\mathrm{q}}\right), 137.1\left(\mathrm{Ar}-\mathrm{C}_{\mathrm{q}}\right), 130.2(\mathrm{Ar}-\mathrm{C}), 128.4(\mathrm{Ar}-\mathrm{C}), 128.3(\mathrm{Ar}-\mathrm{C}), 127.8(\mathrm{Ar}-\mathrm{C}), 120.1(\mathrm{Ar}-$ C), $117.6(\mathrm{Ar}-\mathrm{C}), 112.8(\mathrm{Ar}-\mathrm{C}), 67.7\left(\mathrm{COOCH}_{2}\right), 55.8\left(\mathrm{OCH}_{3}\right), 47.4\left(2 \times \mathrm{NCH}_{2} \mathrm{CH}_{2}\right), 25.7\left(2 \times \mathrm{NCH}_{2} \mathrm{CH}_{2}\right)$. HRMS (ESITOF) m/z (M+Na) calcd for $\mathrm{C}_{19} \mathrm{H}_{22} \mathrm{~N}_{2} \mathrm{NaO}_{3} \mathrm{~S} 381,1249$; found 381,1249.

\section{Isobutyl [(3-methoxyphenyl)(pyrrolidin-1-yl)- $\lambda^{4}$-sulfanylidene]carbamate (4j)}

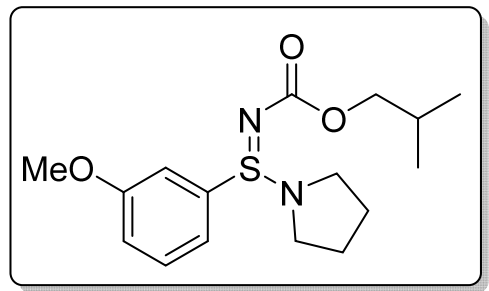

Prepared according to General Procedure D with a reaction time of $2 \mathrm{~h}$, using sulfenamide $1 \mathrm{~h}(104 \mathrm{mg})$ to afford sulfinamidine $\mathbf{4 j}$ as a waxy solid (131 mg, 81\%). $\mathrm{R}_{f} 0.3$ (20\% Hexane in $\mathrm{Et}_{2} \mathrm{O}$ ). IR (film)/cm ${ }^{-1}$ 2959, 2875, 1627, 1472, 1237, 1037, 858. ${ }^{1} \mathrm{H}$ NMR $\left(500 \mathrm{MHz}, \mathrm{CDCl}_{3}, \mathrm{ppm}\right) \delta 7.22(\mathrm{t}$, $J=8.1 \mathrm{~Hz}, 1 \mathrm{H}, \mathrm{Ar}-\mathrm{H}), 6.85-6.84(\mathrm{~m}, 2 \mathrm{H}, \mathrm{Ar}-\mathrm{H}), 6.70-6.68(\mathrm{~m}, 1 \mathrm{H}, \mathrm{Ar}-$ $\mathrm{H}), 3.91\left(2 \times \mathrm{d}, J=6.7 \mathrm{~Hz}, \mathrm{ABX}\right.$ system, $\left.2 \mathrm{H}, 2 \times \mathrm{OCHHCH}\left(\mathrm{CH}_{3}\right)_{2}\right), 3.86$ $\left(\mathrm{s}, 3 \mathrm{H}, \mathrm{OCH}_{3}\right), 3.45-3.40\left(\mathrm{~m}, 2 \mathrm{H}, 2 \times \mathrm{NCHHCH}_{2}\right), 3.19-3.16(\mathrm{~m}, 2 \mathrm{H}, 2$ $\left.\times \mathrm{NCHHCH}_{2}\right), 2.02\left(\right.$ hept, $\left.J=6.7 \mathrm{~Hz}, 1 \mathrm{H}, \mathrm{OCH}_{2} \mathrm{CH}\left(\mathrm{CH}_{3}\right)_{2}\right), 1.88-1.81(\mathrm{~m}, 4 \mathrm{H}, 4 \times \mathrm{CH}), 0.97(\mathrm{~d}, J=6.7$ $\left.\mathrm{Hz}, 6 \mathrm{H}, 2 \times \mathrm{OCH}_{2} \mathrm{CHCH}_{3}\right) .{ }^{13} \mathrm{C}$ NMR $\left(125 \mathrm{MHz}, \mathrm{CDCl}_{3}\right) \delta 166.0$ (NCOO), $160.3\left(\mathrm{Ar}-\mathrm{C}_{\mathrm{q}}\right), 137.3\left(\mathrm{Ar}-\mathrm{C}_{\mathrm{q}}\right)$, $130.2(\mathrm{Ar}-\mathrm{C}), 120.2(\mathrm{Ar}-\mathrm{C}), 117.0(\mathrm{Ar}-\mathrm{C}), 112.9(\mathrm{Ar}-\mathrm{C}), 72.4\left(\mathrm{OCH}_{2} \mathrm{CH}\left(\mathrm{CH}_{3}\right)_{2}\right), 55.8\left(\mathrm{OCH}_{3}\right), 47.4(2 \times$ $\left.\mathrm{NCH}_{2} \mathrm{CH}_{2}\right), 28.3\left(\mathrm{OCH}_{2} \mathrm{CH}\left(\mathrm{CH}_{3}\right)_{2}\right), 25.8\left(2 \times \mathrm{NCH}_{2} \mathrm{CH}_{2}\right), 19.5\left(2 \times \mathrm{OCH}_{2} \mathrm{CHCH}_{3}\right) . \mathrm{HRMS}(\mathrm{ESITOF}) \mathrm{m} / \mathrm{z}$ $(\mathrm{M}+\mathrm{Na})^{+}$calcd for $\mathrm{C}_{16} \mathrm{H}_{24} \mathrm{~N}_{2} \mathrm{NaO}_{3} \mathrm{~S} 347,1405$; found 347,1403 . 


\section{Benzyl [2-naphtyl(morpholin-4-yl)- $\lambda^{4}$-sulfanylidene]carbamate (4k)}

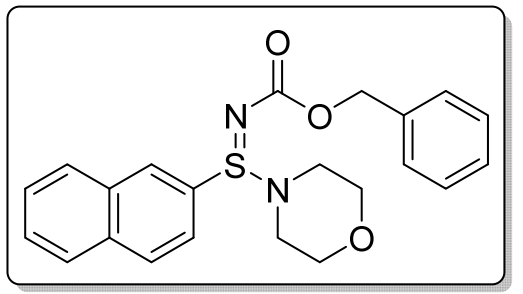

Prepared according to General Procedure D with a reaction time of 2 $\mathrm{h}$, using sulfenamide $\mathbf{1 i}(123 \mathrm{mg})$ to afford sulfinamidine $\mathbf{4 k}$ as a waxy solid (157 mg, 80\%). $\mathrm{R}_{f} 0.25\left(\mathrm{Et}_{2} \mathrm{O}\right)$. IR (film)/ $\mathrm{cm}^{-1} 3058,2957,1633$, 1233, 1107, 1058, 748. ${ }^{1} \mathrm{H} \mathrm{NMR}\left(500 \mathrm{MHz}, \mathrm{CDCl}_{3}, \mathrm{ppm}\right) \delta 8.56(\mathrm{~s}, 1 \mathrm{H}$, Ar-H), $7.96(\mathrm{dd}, J=8.0,4.2 \mathrm{~Hz}, 2 \mathrm{H}, 2 \times \mathrm{Ar}-\mathrm{H}), 7.89(\mathrm{~d}, J=8.0 \mathrm{~Hz}, 1 \mathrm{H}$, Ar-H), 7.77 (dd, $J=8.8,1.8 \mathrm{~Hz}, 1 \mathrm{H}, \mathrm{Ar}-\mathrm{H}), 7.63-7.57(\mathrm{~m}, 2 \mathrm{H}, 2 \times \mathrm{Ar}-$ $\mathrm{H}), 7.47(\mathrm{~d}, J=7.3 \mathrm{~Hz}, 2 \mathrm{H}, 2 \times \mathrm{Ar}-\mathrm{H}), 7.36(\mathrm{t}, J=7.3,2 \mathrm{H}, 2 \times \mathrm{Ar}-\mathrm{H})$, 7.30 (t, $J=7.3,1 \mathrm{H}, \mathrm{Ar}-\mathrm{H}), 5.26$ and $5.22(2 \times \mathrm{d}, J=12.3 \mathrm{~Hz}, \mathrm{AB}$ system, $2 \mathrm{H}, 2 \times \mathrm{OCH} H \mathrm{Ph}), 3.73-3.65$ $\left(\mathrm{m}, 4 \mathrm{H}, 4 \times \mathrm{OCH} \mathrm{CCH}_{2} \mathrm{~N}\right), 3.30-3.25\left(\mathrm{~m}, 2 \mathrm{H}, 2 \times \mathrm{NCHHCH}_{2} \mathrm{O}\right), 3.00-2.95\left(\mathrm{~m}, 2 \mathrm{H}, 2 \times \mathrm{NCHHCH}_{2} \mathrm{O}\right) .{ }^{13} \mathrm{C}$ NMR (125 MHz, CDCl $)_{3}$ ठ $165.2(\mathrm{NCOO}), 137.4\left(\mathrm{Ar}-\mathrm{C}_{\mathrm{q}}\right), 134.6\left(\mathrm{Ar}-\mathrm{C}_{\mathrm{q}}\right), 132.8\left(\mathrm{Ar}-\mathrm{C}_{\mathrm{q}}\right), 130.0\left(\mathrm{Ar}-\mathrm{C}_{\mathrm{q}}\right)$, 129.7 (Ar-C), 129.5 (2 × Ar-C), 129.1 (Ar-C), 128.7 (Ar-C), $128.5(\operatorname{Ar}-\mathrm{C}), 128.4(2 \times \mathrm{Ar}-\mathrm{C}), 127.9(\mathrm{Ar}-$ C), $127.6(\mathrm{Ar}-\mathrm{C}), 123.3(\mathrm{Ar}-\mathrm{C}), 68.0\left(\mathrm{OCH}_{2} \mathrm{Ph}\right), 66.9\left(2 \times \mathrm{OCH}_{2} \mathrm{CH}_{2} \mathrm{~N}\right), 47.0\left(2 \times \mathrm{NCH}_{2} \mathrm{CH}_{2} \mathrm{O}\right)$. HRMS (ESITOF) $\mathrm{m} / \mathrm{z}(\mathrm{M}+\mathrm{Na})^{+}$calcd for $\mathrm{C}_{22} \mathrm{H}_{22} \mathrm{~N}_{2} \mathrm{NaO}_{3} \mathrm{~S} 417,1249$; found 417,1245 .

\section{Benzyl [cyclohexyl(morpholino)- $\lambda^{4}-$ sulfanylidene]carbamate (4I)}

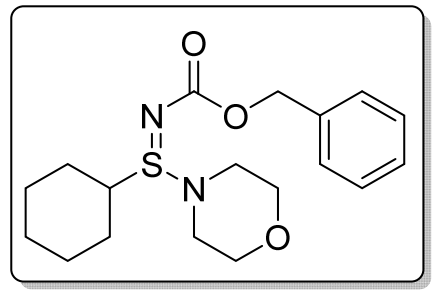

Prepared according to General Procedure D with a reaction time of $24 \mathrm{~h}$, using sulfenamide $1 \mathbf{j}(101 \mathrm{mg})$ to afford sulfinamidine $4 \mathrm{I}$ as a waxy solid (123 $\mathrm{mg}, 70 \%$ ). $\mathrm{R}_{f} 0.5$ (AcOEt). IR (film) $/ \mathrm{cm}^{-1} 2925,2854,1635,1448,1236$, 1084, 916, 858. ${ }^{1} \mathrm{H}$ NMR $\left(300 \mathrm{MHz}, \mathrm{CDCl}_{3}, \mathrm{ppm}\right) \delta 7.42-7.29(\mathrm{~m}, 5 \mathrm{H}, \mathrm{Ar}-$ $\mathrm{H}), 5.11$ and $5.15(2 \times \mathrm{d}, J=12.7 \mathrm{~Hz}, \mathrm{AB}$ system, $2 \mathrm{H}, 2 \times \mathrm{OCHHPh}), 3.73-$ $3.70\left(\mathrm{~m}, 4 \mathrm{H}, 4 \times \mathrm{OCHHCH}_{2} \mathrm{~N}\right), 3.20-3.11(\mathrm{~m}, 5 \mathrm{H}, \mathrm{SCH}$ and $4 \times$ $\left.\mathrm{OCH}_{2} \mathrm{CH} H \mathrm{~N}\right), 2.17-2.14(\mathrm{~m}, 1 \mathrm{H}, \mathrm{CH}), 2.01-1.97(\mathrm{~m}, 1 \mathrm{H}), 1.87-1.83(\mathrm{~m}$, $2 \mathrm{H}), 1.69-1.67(\mathrm{~m}, 2 \mathrm{H}), 1.44-1.24(\mathrm{~m}, 4 \mathrm{H}) .{ }^{13} \mathrm{C} \mathrm{NMR}\left(75 \mathrm{MHz}, \mathrm{CDCl}_{3}\right) \delta 165.3(\mathrm{NCOO}), 137.5\left(\mathrm{Ar}-\mathrm{C}_{\mathrm{q}}\right)$, 128.4 ( $\mathrm{Ar}-\mathrm{C}), 128.2(2 \times \mathrm{Ar}-\mathrm{C}), 127.8\left(\mathrm{Ar}-\mathrm{C}_{\mathrm{q}}\right), 67.7\left(\mathrm{OCH}_{2} \mathrm{Ph}\right), 66.9\left(2 \times \mathrm{OCH}_{2} \mathrm{CH}_{2} \mathrm{~N}\right), 55.1(\mathrm{SCH}), 47.7$ $\left(2 \times \mathrm{NCH}_{2} \mathrm{CH}_{2} \mathrm{O}\right), 27.8\left(\mathrm{CH}_{2}\right), 27.5\left(\mathrm{CH}_{2}\right), 25.4\left(\mathrm{CH}_{2}\right), 25.2\left(\mathrm{CH}_{2}\right), 25.1\left(\mathrm{CH}_{2}\right)$. HRMS (ESITOF) m/z $(\mathrm{M}+\mathrm{Na})^{+}$calcd for $\mathrm{C}_{18} \mathrm{H}_{26} \mathrm{~N}_{2} \mathrm{NaO}_{3} \mathrm{~S} 373,1562$; found 373,1556 .

\section{Isobutyl [cyclohexyl(morpholino)- $\lambda^{4}$-sulfanylidene]carbamate $(4 \mathrm{~m})$}

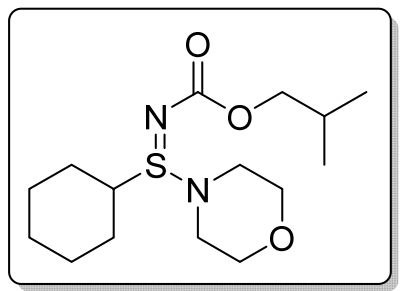

Prepared according to General Procedure D with a reaction time of $24 \mathrm{~h}$, using sulfenamide $1 \mathbf{j}(101 \mathrm{mg})$ to afford sulfinamidine $\mathbf{4 l}$ as a waxy solid (150 $\mathrm{mg}, 95 \%$ ). $\mathrm{R}_{f} 0.5$ (AcOEt). IR (film) $/ \mathrm{cm}^{-1} 2932,2860,1643,1451,1228,1110$, 908, 853. ${ }^{1} \mathrm{H}$ NMR $\left(300 \mathrm{MHz}, \mathrm{CDCl}_{3}, \mathrm{ppm}\right) \delta 3.89$ and $3.81(2 \times \mathrm{dd}, J=10.4$, $6.9 \mathrm{~Hz}, \mathrm{ABX}$ system, $\left.2 \mathrm{H}, 2 \times \mathrm{OCHHCH}\left(\mathrm{CH}_{3}\right)_{2}\right), 3.75-3.72(\mathrm{~m}, 4 \mathrm{H}, 4 \times$ $\left.\mathrm{OCH} \mathrm{HCH}_{2} \mathrm{~N}\right), 3.17-3.14\left(\mathrm{~m}, 4 \mathrm{H}, 4 \times \mathrm{OCH}_{2} \mathrm{CHHN}\right), 2.20-2.13(\mathrm{~m}, 1 \mathrm{H}, \mathrm{CH})$, 2.03-1.94 (m, 1H), 1.90-1.80 (m, 2H), 1.70-1.65 (m, 2H), 1.46-1.24 (m, 4H), $0.94\left(\mathrm{~d}, J=6.6 \mathrm{~Hz}, 6 \mathrm{H}, 2 \times \mathrm{OCH}_{2} \mathrm{CHCH}_{3}\right) .{ }^{13} \mathrm{C} \mathrm{NMR}\left(75 \mathrm{MHz}, \mathrm{CDCl}_{3}\right) \delta 166.0(\mathrm{NCOO}), 72.3$ $\left(\mathrm{OCH}_{2} \mathrm{CH}\left(\mathrm{CH}_{3}\right)_{2}\right), 66.9\left(2 \times \mathrm{OCH}_{2} \mathrm{CH}_{2} \mathrm{~N}\right), 54.8(\mathrm{SCH}), 47.6\left(2 \times \mathrm{NCH}_{2} \mathrm{CH}_{2} \mathrm{O}\right), 28.3\left(\mathrm{OCH}_{2} \mathrm{CH}\left(\mathrm{CH}_{3}\right)_{2}\right), 27.9$ $\left(\mathrm{CH}_{2}\right), 27.6\left(2 \times \mathrm{CH}_{2}\right), 25.4\left(\mathrm{CH}_{2}\right), 25.2\left(\mathrm{CH}_{2}\right), 25.1\left(\mathrm{CH}_{2}\right), 19.4\left(2 \times \mathrm{OCH}_{2} \mathrm{CHCH}_{3}\right)$. HRMS (ESITOF) $\mathrm{m} / \mathrm{z}$ $(\mathrm{M}+\mathrm{Na})^{+}$calcd for $\mathrm{C}_{15} \mathrm{H}_{28} \mathrm{~N}_{2} \mathrm{NaO}_{3} \mathrm{~S} 339,1718$; found 339, 1716 .

\section{Benzyl (3-oxo-2,3-dihydro-1 $\lambda^{4}-$ benzo[d]isothiazol-1-ylidene)carbamate (4n)}

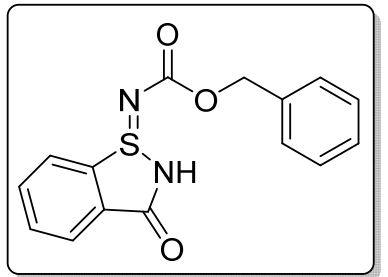

Prepared according to General Procedure D using sulfenamide $1 \mathbf{k}(76 \mathrm{mg})$ to afford sulfinamidine $4 \mathrm{n}$ as a white waxy solid $(113 \mathrm{mg}, 75 \%) . \mathrm{R}_{f} 0.3$ (AcOEt). IR (film) $/ \mathrm{cm}^{-1}$ 3031, 2668, 1718, 1642, 1261, 1213, 868, 750. ${ }^{1} \mathrm{H}$ NMR (300 $\left.\mathrm{MHz}, \mathrm{CDCl}_{3}, \mathrm{ppm}\right) \delta 8.03$ (d, J = 6.8 Hz, 1H, Ar-H), 7.87-7.76 (m, 3H, Ar-H), 7.33-7.22 (m overlapping $\left.\mathrm{CDCl}_{3}, 5 \mathrm{H}, \mathrm{Ar}-\mathrm{H}\right), 5.11\left(\mathrm{~s}, 2 \mathrm{H}, \mathrm{OCH}_{2} \mathrm{Ph}\right) \cdot{ }^{13} \mathrm{C} \mathrm{NMR}$ $\left(75 \mathrm{MHz}, \mathrm{CDCl}_{3}\right) \delta 168.5,164.2,136.3,134.6,133.4,128.5,128.3,128.2$, 126.7, 125.8, $68.7\left(\mathrm{COCH}_{2} \mathrm{Ph}\right)$. HRMS (ESITOF) $\mathrm{m} / \mathrm{z}(\mathrm{M}-\mathrm{H})^{-}$calcd for $\mathrm{C}_{15} \mathrm{H}_{11} \mathrm{~N}_{2} \mathrm{O}_{3} \mathrm{~S}$ 299,0496; found 299,0493. 
Isobutyl (3-oxo-2,3-dihydro-1 $\Lambda^{4}-$ benzo[ $[d]$ isothiazol-1-ylidene)carbamate (40)

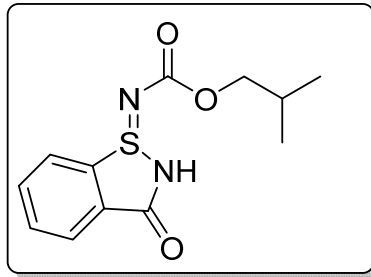

Prepared according to General Procedure D using sulfenamide $1 \mathbf{k}(76 \mathrm{mg})$ to afford sulfinamidine $4 \mathrm{n}$ as a white waxy solid $(120 \mathrm{mg}, 90 \%) . \mathrm{R}_{f} 0.3$ (AcOEt). IR (film)/cm-1 2961, 2927, 2348, 1721, 1648, 1457, 1222, 753. ${ }^{1} \mathrm{H}$ NMR (300 $\left.\mathrm{MHz}, \mathrm{CDCl}_{3}, \mathrm{ppm}\right) \delta 8.10-8.07(\mathrm{~m}, 1 \mathrm{H}, \mathrm{Ar}-\mathrm{H}), 7.94-7.79(\mathrm{~m}, 3 \mathrm{H}, \mathrm{Ar}-\mathrm{H}), 3.91$ and $3.87\left(2 \times \mathrm{dd}, J=10.5,6.6 \mathrm{~Hz}, \mathrm{ABX}\right.$ system, $\left.2 \mathrm{H}, 2 \times \mathrm{OCHHCH}\left(\mathrm{CH}_{3}\right)_{2}\right)$, 2.00-1.87 (m, $\left.1 \mathrm{H}, \mathrm{OCH}_{2} \mathrm{CH}\left(\mathrm{CH}_{3}\right)_{2}\right), 0.92\left(\mathrm{~d}, J=6.7 \mathrm{~Hz}, 6 \mathrm{H}, \mathrm{CH}\left(\mathrm{CH}_{3}\right)_{2}\right) .{ }^{13} \mathrm{C}$ $\operatorname{NMR}\left(75 \mathrm{MHz}, \mathrm{CDCl}_{3}\right) \delta$ 167.8, 163.8, $140.4\left(\mathrm{Ar}-\mathrm{C}_{\mathrm{q}}\right), 134.8(\mathrm{Ar}-\mathrm{C}), 133.5(\mathrm{Ar}-$ C), $\left.129.0\left(\mathrm{Ar}-\mathrm{C}_{\mathrm{q}}\right), 126.8(\mathrm{Ar}-\mathrm{C}), 125.9(\mathrm{Ar}-\mathrm{C}), 73.4\left(\mathrm{COCH}_{2} \mathrm{CH}\left(\mathrm{CH}_{3}\right)_{2}\right)\right), 28.0\left(\mathrm{COCH}_{2} \mathrm{CH}\left(\mathrm{CH}_{3}\right)_{2}\right), 19.2$ $\left(2 \times \mathrm{CH}_{3}\right)$. HRMS (ESITOF) m/z (M+H)- calcd for $\mathrm{C}_{12} \mathrm{H}_{13} \mathrm{~N}_{2} \mathrm{O}_{3} \mathrm{~S} 265,0652$; found 265,0652 . 


\section{Mechanistic investigation}

\subsection{Control experiments}
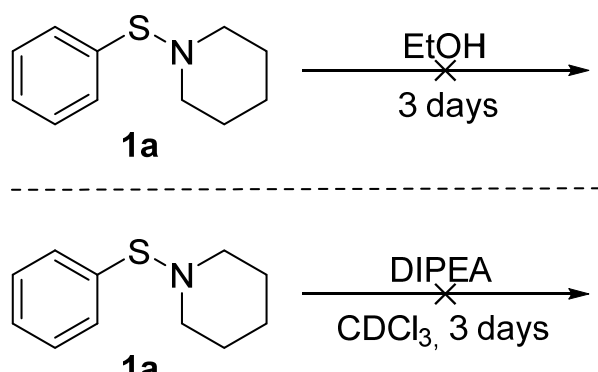

$1 \mathrm{a}$

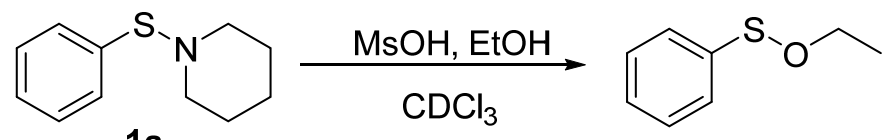

$1 \mathrm{a}$
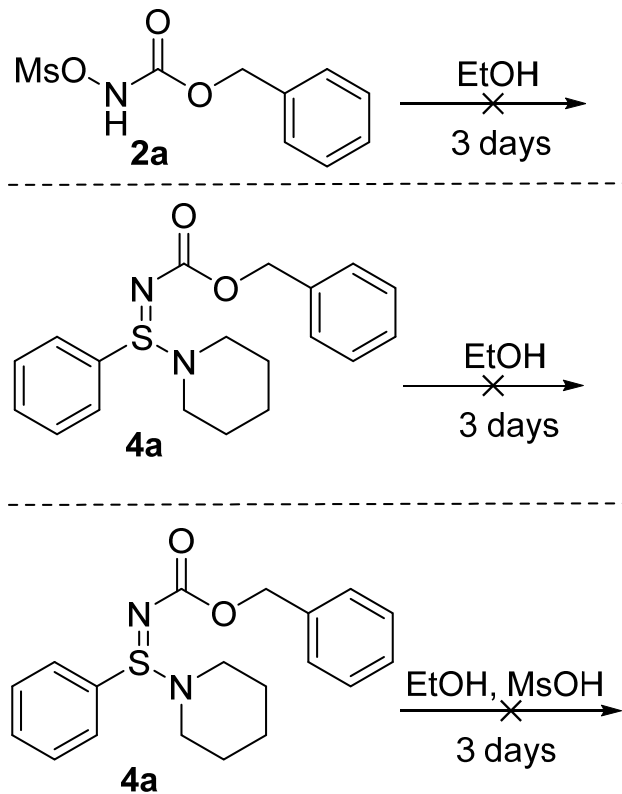

Sulfenamide 1a was found to be stable in ethanol and in the presence of DIPEA in deuterated chloroform. On the other hand, treatment of 1a with methanesulfonic acid resulted in the formation of ethoxy(phenyl)sulfane. $\mathrm{N}$-source $\mathbf{2 a}$ was stable in the presence of DIPEA, and no formation of sulfinimidate ester 3a was observed when sulfinamidine $\mathbf{4 a}$ was treated with ethanol, even in the presence of methanesolfonic acid. 


\section{$7.2{ }^{1} \mathrm{H}$ NMR experiments: formation of $3 \mathrm{a}$}

${ }^{1} \mathrm{H}$ NMR Mechanistic investigation: Ethyl N-((benzyloxy)carbonyl)phenylsulfinimidate (3a)

The formation of ethyl $\mathrm{N}-(($ benzyloxy)carbonyl)phenylsulfinimidate 3a was monitored in a NMR tube by addition of benzyl((methylsulfonyl)oxy)carbamate $2 \mathrm{a}$ (1 equiv.) to a solution of sulfenamide $1 \mathrm{a}$ (1 equiv.) and ethanol (2 equiv.) in $\mathrm{CDCl}_{3}$.
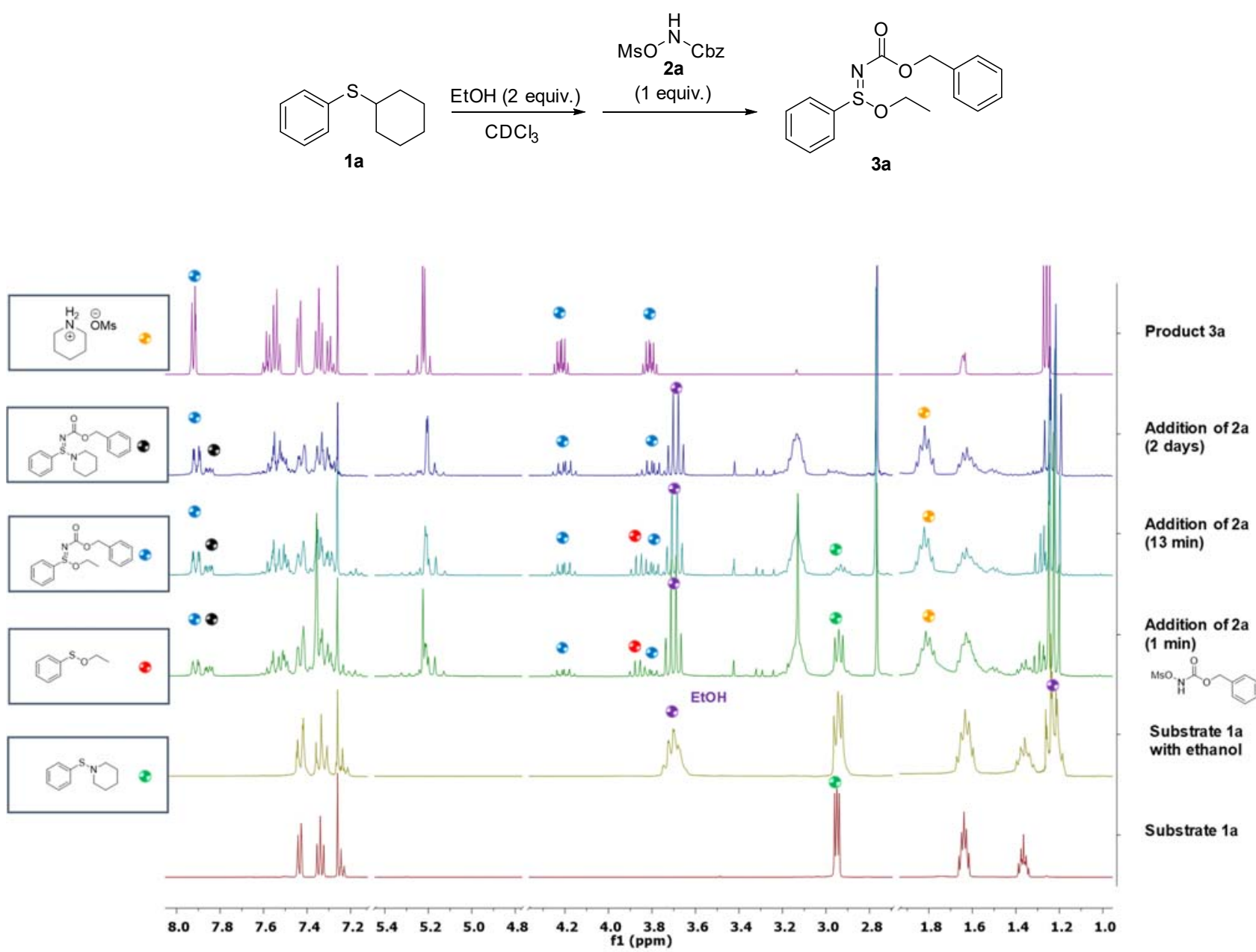
The formation of ethyl $\mathrm{N}-(($ benzyloxy)carbonyl)phenylsulfinimidate 3a was monitored in a NMR tube by addition of sulfenamide 1a (1 equiv.) to a solution of ethanol (2 equiv.) and methanesulfonic acid in $\mathrm{CDCl}_{3}$, and the subsequent addition of benzyl((methylsulfonyl)oxy)carbamate $2 \mathrm{a}$ (1 equiv.).
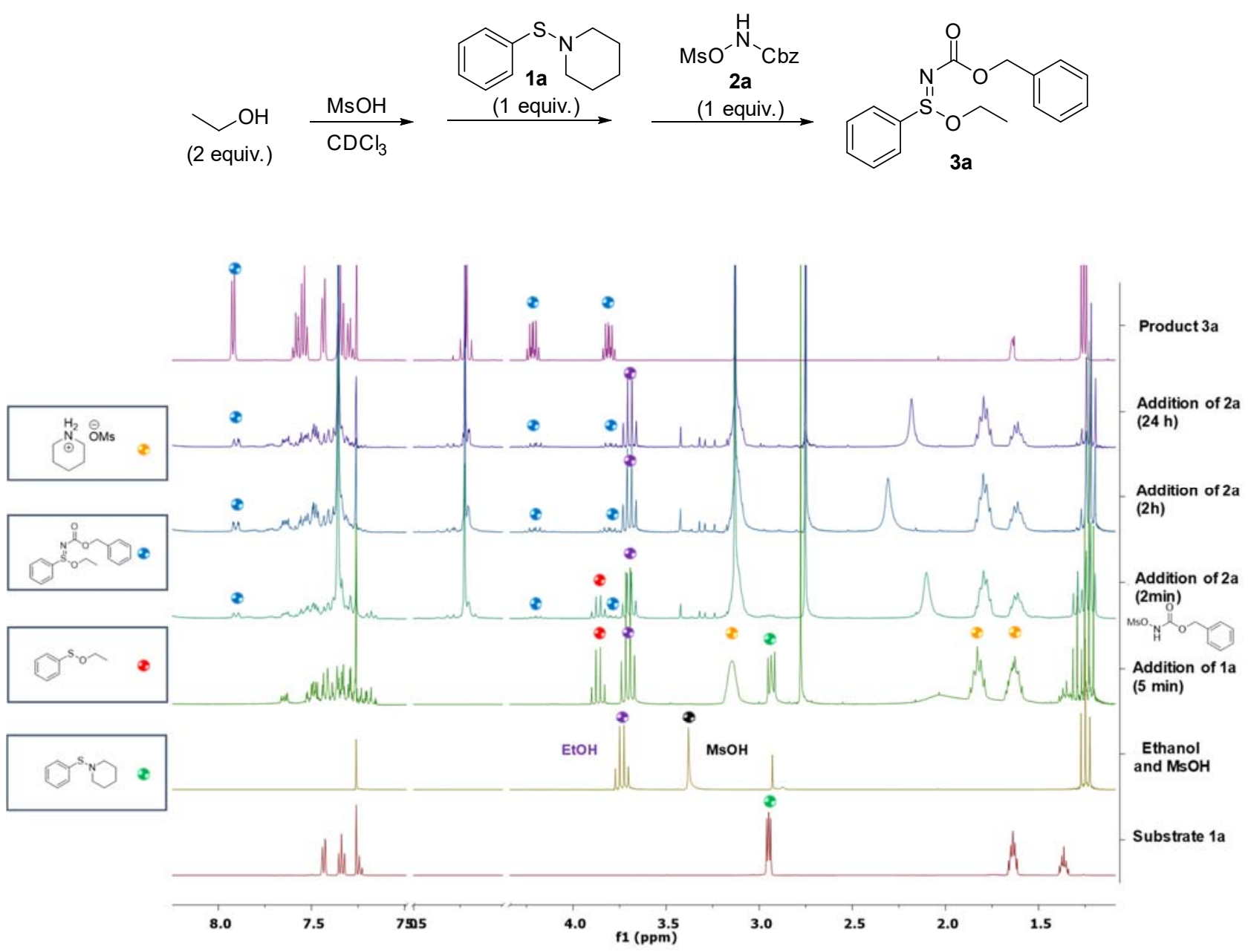
The formation of ethyl $\mathrm{N}-(($ benzyloxy)carbonyl)phenylsulfinimidate 3a was monitored in a NMR tube by addition of benzyl((methylsulfonyl)oxy)carbamate $2 \mathrm{a}$ (1 equiv.) to a solution of sulfenamide $1 \mathrm{a}$ (1 equiv.) in $\mathrm{CDCl}_{3}$. Subsequently, ethanol (2 equiv.) was added in one portion.
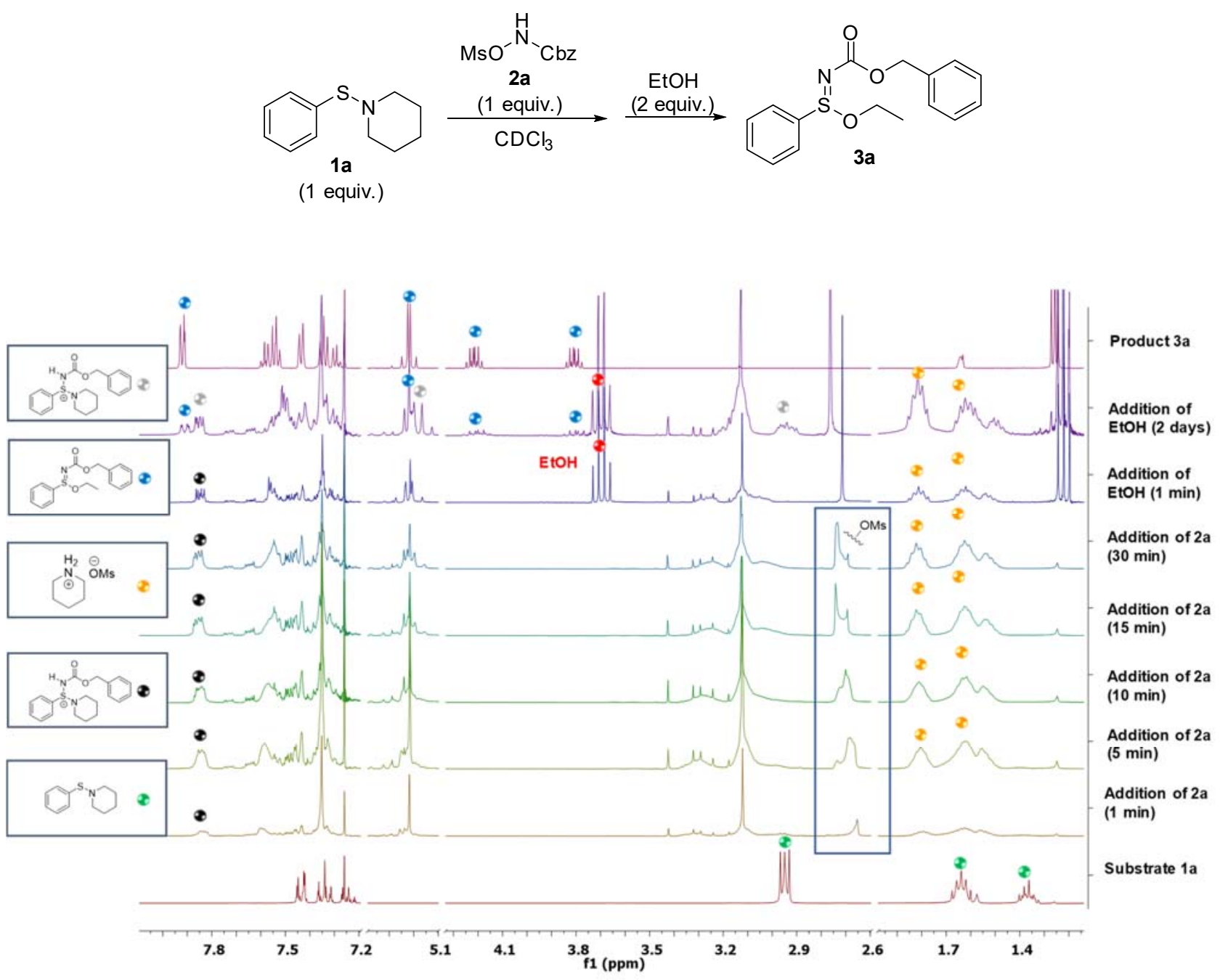


\section{$7.3^{1} \mathrm{H}$ NMR experiment: formation of $4 \mathrm{a}$}

${ }^{1} \mathrm{H}$ NMR Mechanistic investigation: formation of benzyl-(phenyl(piperidin-1-yl)- $\lambda^{4}-$ sulfanylidene)carbamate (4a)

The formation of benzyl-(phenyl(piperidin-1-yl)- $\lambda^{4}$-sulfanylidene)carbamate $4 a$ was monitored in a NMR by addition of benzyl((methylsulfonyl)oxy)carbamate $2 a$ to a solution of sulfenamide $1 a$ in $\mathrm{CDCl}_{3}$. Subsequently, DIPEA was added in one portion.

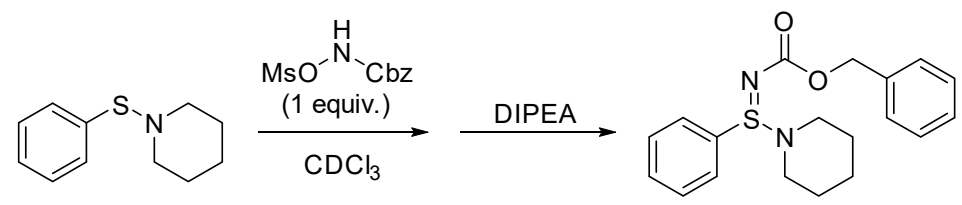

$1 a$

4a

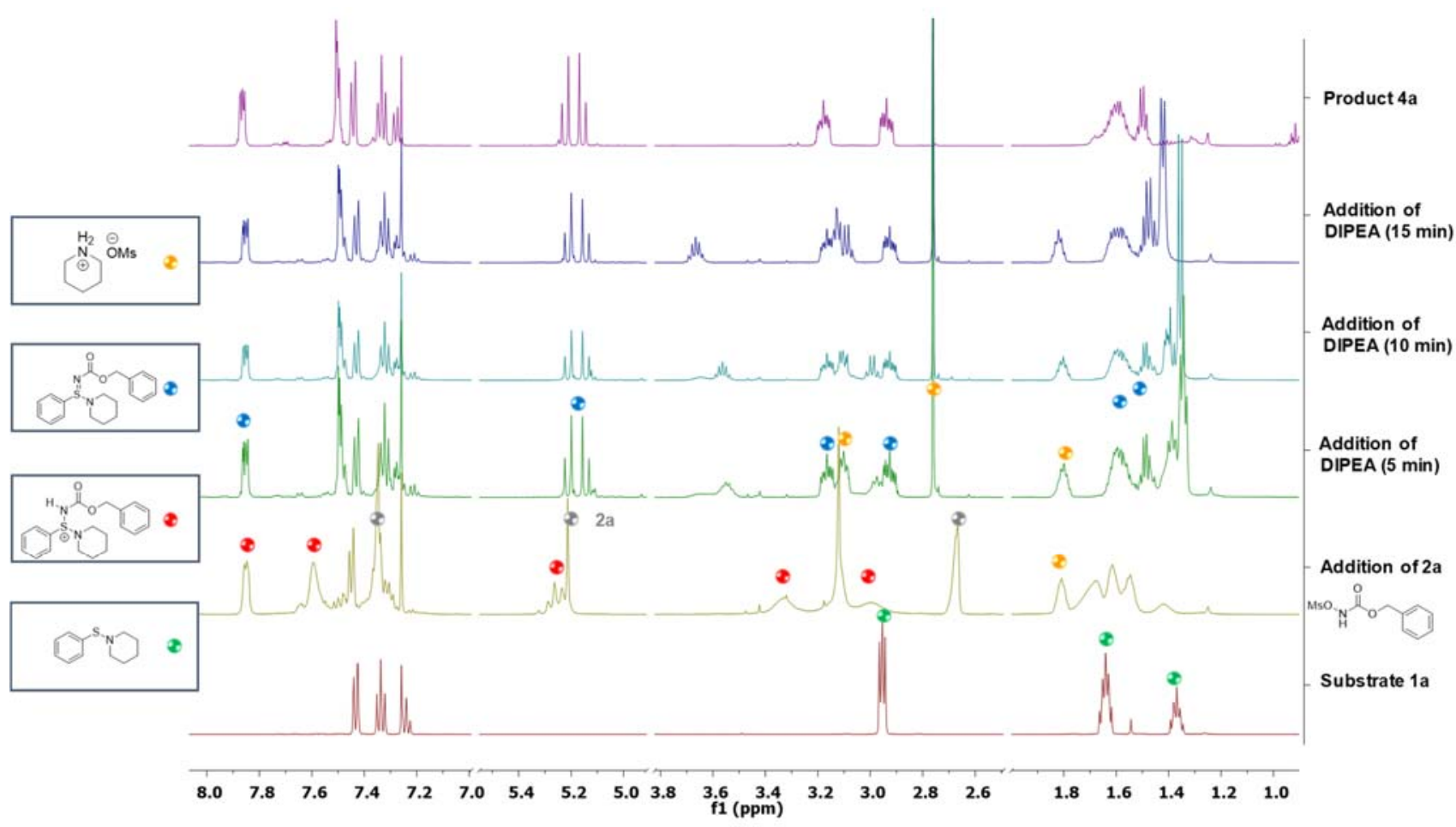




\subsection{Ab initio Computational Calculations}

\section{Reaction pathway for the formation of sulfinamidine}

All the computations were run using DFT-B3LYP method with def2-tzvp basis set.

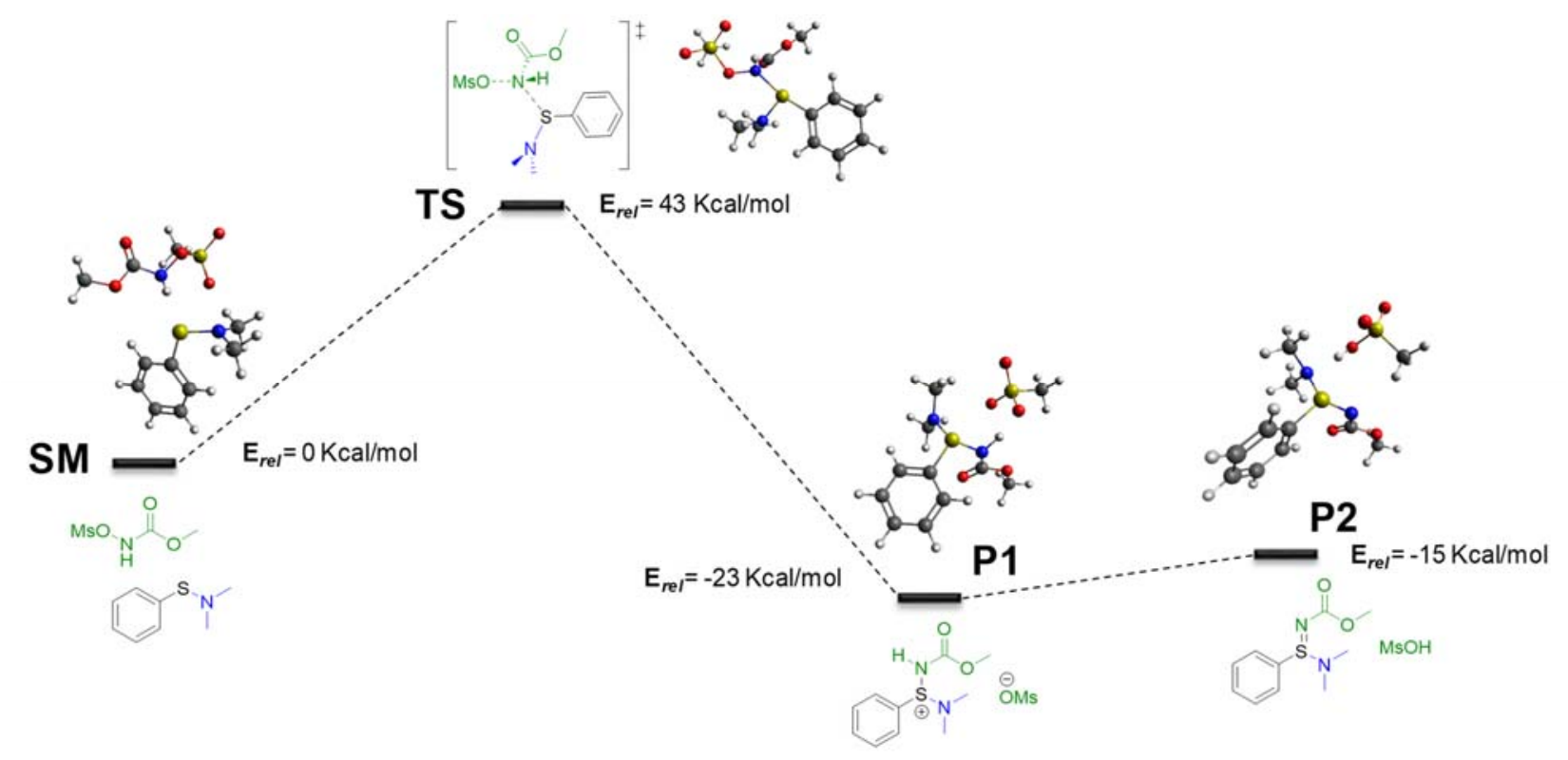

Reaction pathway for the formation of sulfinimidate ester

All the computations were run using DFT-B3LYP method with def2-tzvp basis set. The solvent (methanol) was modelized using COSMO model.

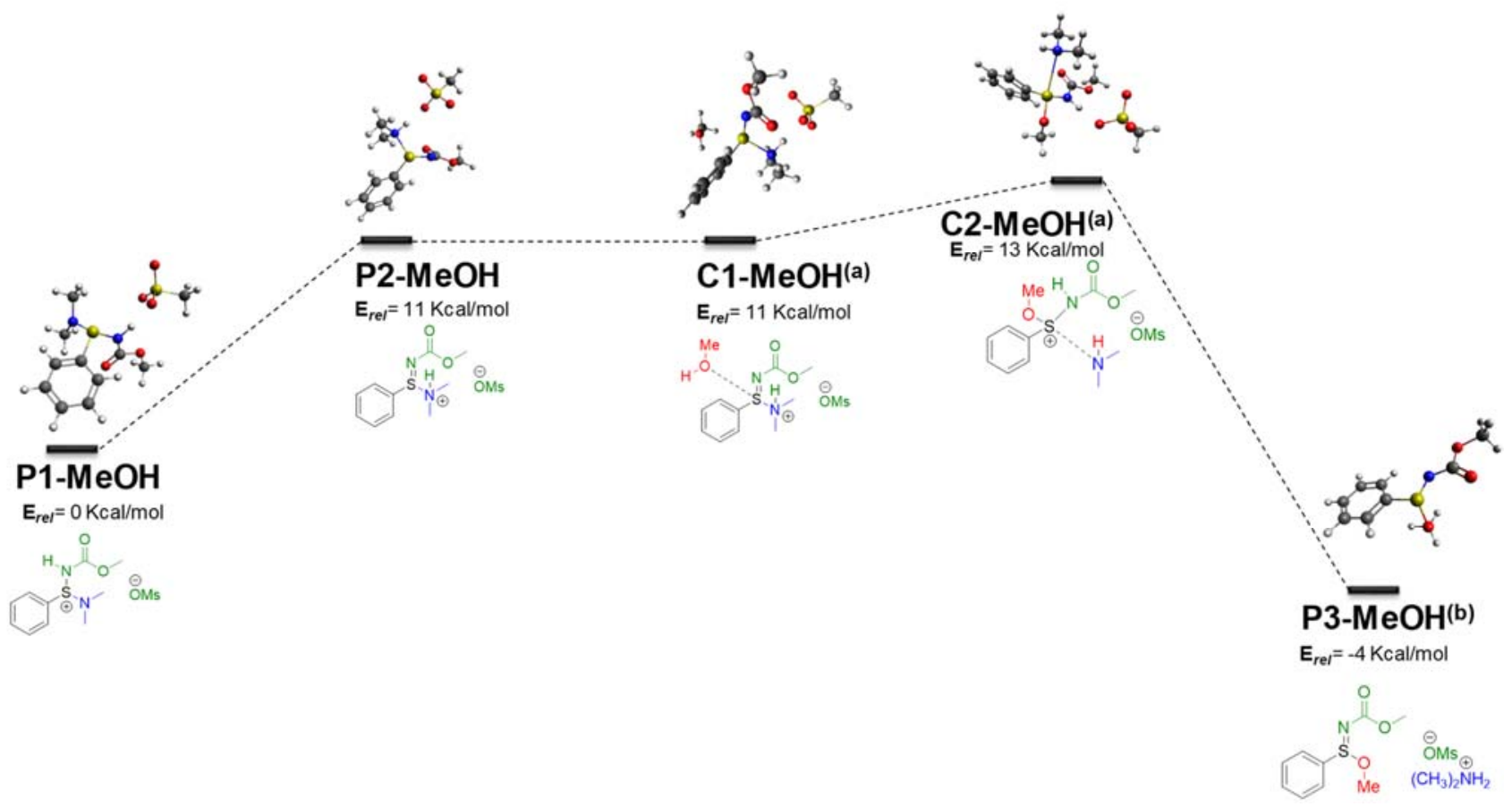

(a) Energy computed by subtraction of free $\mathrm{MeOH}$ calculated energy.

(b) Energy computed by subtraction of free $\mathrm{MeOH}$ calculated energy and addition of dimethylammonium and mesylate calculated energies in condition of perfect dilution. 
Equilibrium structure search and transition state geometries

Equilibrium structure search and transition state geometries

SM

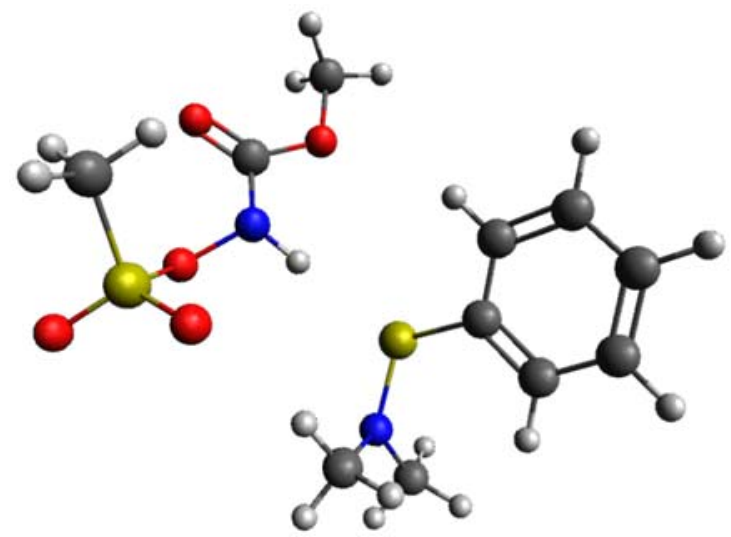

Energy $-46595.914 \mathrm{eV}$, i.e. $-1074527.029 \mathrm{Kcal} / \mathrm{mol} ; \mathbf{E}_{\mathrm{rel}}=0 \mathrm{Kcal} / \mathrm{mol}$

Number of imaginary frequencies $=0$

Coordinates $(\mathbf{x}, \mathbf{y}, \mathbf{z})$

$\begin{array}{llll}\mathrm{H} & 0.70914 & 0.16569 & 3.30833 \\ \mathrm{C} & 1.38631 & -0.31327 & 2.61195 \\ \mathrm{C} & 2.43896 & -1.08933 & 3.08600 \\ \mathrm{H} & 2.58824 & -1.21392 & 4.15053 \\ \mathrm{C} & 3.29354 & -1.70731 & 2.18072 \\ \mathrm{H} & 4.11560 & -2.31494 & 2.53826 \\ \mathrm{C} & 3.10794 & -1.54822 & 0.81185 \\ \mathrm{H} & 3.78598 & -2.02752 & 0.12057 \\ \mathrm{C} & 2.04961 & -0.77485 & 0.33819 \\ \mathrm{C} & 1.18415 & -0.16133 & 1.24662 \\ \mathrm{H} & 0.34806 & 0.42538 & 0.88481 \\ \mathrm{~S} & 1.73261 & -0.48320 & -1.40227 \\ \mathrm{~N} & 2.50049 & -1.65635 & -2.30629 \\ \mathrm{C} & 2.05159 & -3.04240 & -2.23847 \\ \mathrm{H} & 2.54517 & -3.60820 & -1.43831 \\ \mathrm{H} & 0.97728 & -3.08374 & -2.07503 \\ \mathrm{C} & 3.88891 & -1.46013 & -2.70244 \\ \mathrm{H} & 4.60783 & -1.89948 & -1.99838 \\ \mathrm{H} & 4.09706 & -0.39587 & -2.78975 \\ \mathrm{H} & 2.27611 & -3.53249 & -3.18910 \\ \mathrm{H} & 4.04850 & -1.92651 & -3.67826 \\ \mathrm{O} & -1.56029 & -3.58833 & -1.72418 \\ \mathrm{H} & -4.44869 & -3.69342 & -1.61989 \\ \mathrm{H} & -3.84294 & -2.19775 & -0.85260 \\ \mathrm{~S} & -2.48304 & -2.96503 & -2.62536 \\ \mathrm{C} & -4.03003 & -2.70384 & -1.79566 \\ \mathrm{H} & -0.61600 & -0.65107 & -1.66841 \\ \mathrm{~N} & -1.63157 & -0.71577 & -1.76683 \\ \mathrm{O} & -1.71131 & 1.25975 & -0.76602 \\ \mathrm{O} & -2.70529 & -3.47748 & -3.93937\end{array}$




$\begin{array}{rrrr}\mathrm{H} & -3.32395 & 2.18758 & 0.17662 \\ \mathrm{C} & -2.39197 & 0.42595 & -1.57781 \\ \mathrm{O} & -1.97348 & -1.42564 & -2.92925 \\ \mathrm{H} & -1.73120 & 2.99579 & 0.26417 \\ \mathrm{C} & -2.41964 & 2.44724 & -0.37219 \\ \mathrm{H} & -4.67201 & -2.11092 & -2.44082 \\ \mathrm{O} & -3.49825 & 0.61510 & -2.01034 \\ \mathrm{H} & -2.68534 & 3.03875 & -1.24668\end{array}$

TS

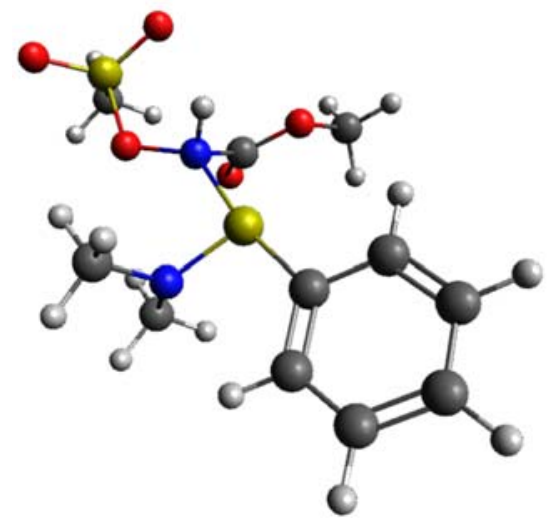

Energy $-46594.032 \mathrm{eV}$, i.e. $-1074483.629 \mathrm{Kcal} / \mathrm{mol} ; \mathbf{E}_{\mathrm{rel}}=43.400 \mathrm{Kcal} / \mathrm{mol}$ Number of imaginary frequencies $=1$

Coordinates $(x, y, z)$

$\begin{array}{llll}\mathrm{H} & 2.49029 & 0.21001 & 4.46452 \\ \mathrm{C} & 2.86733 & -0.16835 & 3.52183 \\ \mathrm{C} & 4.23372 & -0.36960 & 3.34720 \\ \mathrm{H} & 4.92571 & -0.14920 & 4.14999 \\ \mathrm{C} & 4.69888 & -0.86039 & 2.13302 \\ \mathrm{H} & 5.76013 & -1.02337 & 1.98652 \\ \mathrm{C} & 3.81303 & -1.14749 & 1.09835 \\ \mathrm{H} & 4.17915 & -1.53136 & 0.15604 \\ \mathrm{C} & 2.44543 & -0.94603 & 1.26816 \\ \mathrm{C} & 1.98204 & -0.45510 & 2.49151 \\ \mathrm{H} & 0.91789 & -0.30016 & 2.63916 \\ \mathrm{~S} & 1.15686 & -1.25430 & 0.01579 \\ \mathrm{~N} & 2.11816 & -1.88684 & -1.21667 \\ \mathrm{C} & 2.07599 & -3.32534 & -1.44819 \\ \mathrm{H} & 2.99130 & -3.62557 & -1.96647 \\ \mathrm{H} & 2.04295 & -3.84601 & -0.49249 \\ \mathrm{C} & 2.34836 & -1.06703 & -2.39737 \\ \mathrm{H} & 3.24272 & -1.43221 & -2.91038 \\ \mathrm{H} & 2.52509 & -0.03538 & -2.09719 \\ \mathrm{H} & 1.22165 & -3.64591 & -2.05587 \\ \mathrm{H} & 1.51687 & -1.08575 & -3.11250 \\ \mathrm{O} & -3.17304 & -2.19397 & -1.64425 \\ \mathrm{H} & -3.76419 & -1.68720 & -4.45556 \\ \mathrm{H} & -2.65833 & -0.42300 & -3.80813\end{array}$




$\begin{array}{lrrr}\mathrm{S} & -2.37428 & -2.53710 & -2.78959 \\ \mathrm{C} & -2.74727 & -1.45169 & -4.14474 \\ \mathrm{H} & -1.05195 & -1.95766 & -0.46264 \\ \mathrm{~N} & -0.53280 & -1.42991 & -1.16285 \\ \mathrm{O} & -1.49479 & 0.28476 & -0.03655 \\ \mathrm{O} & -2.30970 & -3.87810 & -3.28014 \\ \mathrm{H} & -2.75194 & 1.86324 & -0.55530 \\ \mathrm{C} & -1.07429 & -0.08483 & -1.23841 \\ \mathrm{O} & -0.81899 & -2.12724 & -2.43478 \\ \mathrm{H} & -2.17498 & 1.78312 & 1.13604 \\ \mathrm{C} & -1.90067 & 1.66387 & 0.09280 \\ \mathrm{H} & -2.04503 & -1.66357 & -4.94735 \\ \mathrm{O} & -1.07205 & 0.57553 & -2.23769 \\ \mathrm{H} & -1.07371 & 2.32174 & -0.16798\end{array}$

P1

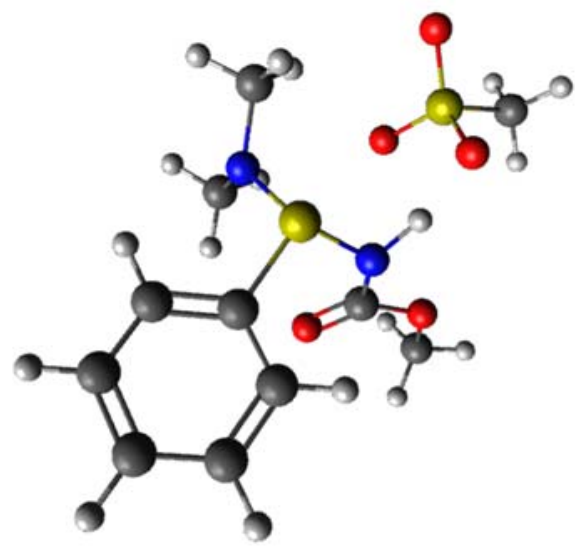

Energy $-46596.898 \mathrm{eV},-1074549.721 \mathrm{Kcal} / \mathrm{mol} ; \mathbf{E}_{\text {rel }}=-22.692 \mathrm{Kcal} / \mathrm{mol}$

\section{Number of imaginary frequencies $=0$}

\section{Coordinates $(x, y, z)$}

$\begin{array}{llrl}\mathrm{H} & 1.88900 & 0.07840 & 4.27466 \\ \mathrm{C} & 2.37561 & -0.33605 & 3.40148 \\ \mathrm{C} & 3.76387 & -0.37326 & 3.32405 \\ \mathrm{H} & 4.36174 & 0.01541 & 4.13807 \\ \mathrm{C} & 4.38151 & -0.91387 & 2.20351 \\ \mathrm{H} & 5.46118 & -0.95051 & 2.14003 \\ \mathrm{C} & 3.61892 & -1.41590 & 1.15510 \\ \mathrm{H} & 4.09631 & -1.85036 & 0.28925 \\ \mathrm{C} & 2.23359 & -1.34779 & 1.23382 \\ \mathrm{C} & 1.60180 & -0.82566 & 2.35909 \\ \mathrm{H} & 0.52339 & -0.79498 & 2.41920 \\ \mathrm{~S} & 1.23113 & -2.15258 & -0.01981 \\ \mathrm{~N} & 2.19320 & -2.19925 & -1.33638 \\ \mathrm{C} & 2.01947 & -3.39435 & -2.19100 \\ \mathrm{H} & 3.00404 & -3.67322 & -2.56965 \\ \mathrm{H} & 1.61998 & -4.21731 & -1.60264 \\ \mathrm{C} & 2.62167 & -0.99577 & -2.06514\end{array}$




$\begin{array}{rrrr}\mathrm{H} & 3.55283 & -1.23677 & -2.58029 \\ \mathrm{H} & 2.78498 & -0.17159 & -1.37874 \\ \mathrm{H} & 1.33698 & -3.18964 & -3.01465 \\ \mathrm{H} & 1.86125 & -0.71680 & -2.79683 \\ \mathrm{O} & -1.83221 & -2.66301 & -1.34050 \\ \mathrm{H} & -3.88540 & -2.80804 & -3.27232 \\ \mathrm{H} & -3.39461 & -1.09817 & -3.09117 \\ \mathrm{~S} & -1.57273 & -2.59482 & -2.81282 \\ \mathrm{C} & -3.12896 & -2.06452 & -3.51434 \\ \mathrm{H} & -0.93410 & -1.75576 & -0.54736 \\ \mathrm{~N} & -0.10910 & -1.19894 & -0.09810 \\ \mathrm{O} & -1.34012 & 0.61361 & -0.44148 \\ \mathrm{O} & -1.27290 & -3.89216 & -3.38692 \\ \mathrm{H} & -2.56276 & 2.17886 & -0.78960 \\ \mathrm{C} & -0.08568 & 0.18420 & -0.29520 \\ \mathrm{O} & -0.59578 & -1.54404 & -3.11811 \\ \mathrm{H} & -1.07127 & 2.60964 & 0.09963 \\ \mathrm{C} & -1.49171 & 2.01719 & -0.71167 \\ \mathrm{H} & -3.00414 & -1.98812 & -4.59262 \\ \mathrm{O} & 0.89716 & 0.88445 & -0.29012 \\ \mathrm{H} & -0.99695 & 2.27544 & -1.64665\end{array}$

P2

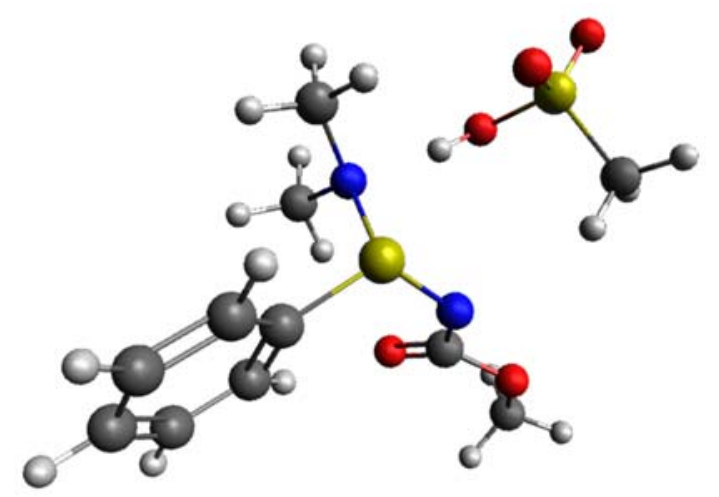

Energy $-46596.574 \mathrm{eV},-1074542.249 \mathrm{Kcal} / \mathrm{mol} ; \mathrm{E}_{\mathrm{rel}}=-15.220 \mathrm{Kcal} / \mathrm{mol}$

Number of imaginary frequencies $=0$

Coordinates $(\mathbf{x}, \mathbf{y}, \mathbf{z})$

$\begin{array}{lrrr}\mathrm{H} & 3.69718 & 1.25893 & 2.50999 \\ \mathrm{C} & 3.42431 & 0.23345 & 2.29580 \\ \mathrm{C} & 4.04422 & -0.80901 & 2.97815 \\ \mathrm{H} & 4.79912 & -0.59429 & 3.72365 \\ \mathrm{C} & 3.68986 & -2.12486 & 2.70822 \\ \mathrm{H} & 4.16140 & -2.93944 & 3.24243 \\ \mathrm{C} & 2.71418 & -2.39723 & 1.75676 \\ \mathrm{H} & 2.41651 & -3.41970 & 1.56040 \\ \mathrm{C} & 2.10332 & -1.34846 & 1.06721 \\ \mathrm{C} & 2.45403 & -0.02442 & 1.33555 \\ \mathrm{H} & 1.96805 & 0.78174 & 0.80813\end{array}$




$\begin{array}{lrrr}\mathrm{S} & 0.85544 & -1.91903 & -0.11492 \\ \mathrm{~N} & 1.64278 & -1.78486 & -1.66610 \\ \mathrm{C} & 2.37195 & -3.03187 & -1.97033 \\ \mathrm{H} & 3.29285 & -3.12534 & -1.38414 \\ \mathrm{H} & 1.72441 & -3.88824 & -1.79812 \\ \mathrm{C} & 2.43482 & -0.58467 & -1.98255 \\ \mathrm{H} & 3.38064 & -0.56579 & -1.43156 \\ \mathrm{H} & 1.86110 & 0.30807 & -1.75639 \\ \mathrm{H} & 2.63715 & -3.01224 & -3.02758 \\ \mathrm{H} & 2.65633 & -0.61072 & -3.05087 \\ \mathrm{~N} & -0.48854 & -1.09495 & -0.08823 \\ \mathrm{O} & -1.91537 & 0.58744 & -0.10388 \\ \mathrm{H} & -3.30253 & 2.04883 & -0.13243 \\ \mathrm{C} & -0.61228 & 0.26092 & -0.23553 \\ \mathrm{H} & -1.71982 & 2.56908 & 0.51343 \\ \mathrm{C} & -2.22436 & 1.97823 & -0.25083 \\ \mathrm{O} & 0.24404 & 1.10110 & -0.46309 \\ \mathrm{H} & -1.92473 & 2.33959 & -1.23431 \\ \mathrm{H} & 0.24093 & -1.78974 & -2.85086 \\ \mathrm{C} & -2.75712 & -2.41284 & -2.56622 \\ \mathrm{H} & -2.40052 & -2.07714 & -1.59389 \\ \mathrm{H} & -3.46777 & -3.23076 & -2.45989 \\ \mathrm{H} & -3.20214 & -1.59456 & -3.12754 \\ \mathrm{O} & -1.82012 & -3.33937 & -4.82779 \\ \mathrm{O} & -0.42296 & -1.78366 & -3.59633 \\ \mathrm{O} & -0.71858 & -4.05489 & -2.71004 \\ \mathrm{~S} & -1.38283 & -3.04611 & -3.49729\end{array}$

Reaction pathway for the formation of sulfinimidate ester P1-MeOH

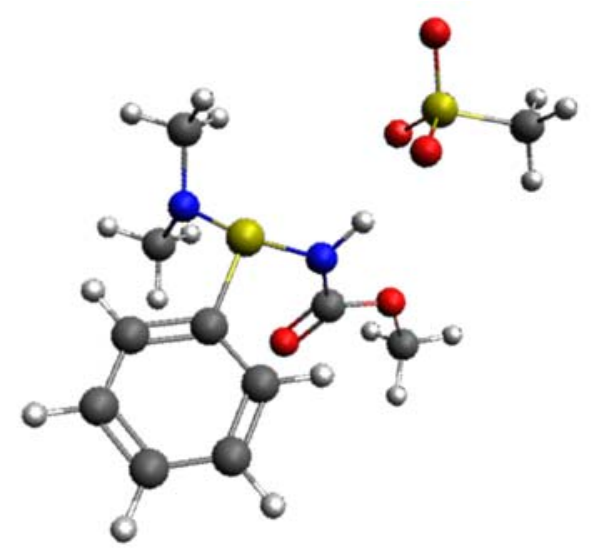

Energy $-46598.532 \mathrm{eV}$, i.e. $-1074587.402 \mathrm{Kcal} / \mathrm{mol}$; $\mathbf{E}_{\text {rel }}=0 \mathrm{Kcal} / \mathrm{mol}$

Number of imaginary frequencies $=0$

Coordinates $(x, y, z)$

$\begin{array}{llll}H & 1.40988 & 0.04099 & 4.11564\end{array}$ 


$\begin{array}{rrrr}\mathrm{C} & 2.04254 & -0.39914 & 3.35570 \\ \mathrm{C} & 3.41046 & -0.53134 & 3.57070 \\ \mathrm{H} & 3.84701 & -0.19004 & 4.50086 \\ \mathrm{C} & 4.21726 & -1.10677 & 2.59566 \\ \mathrm{H} & 5.28092 & -1.21882 & 2.76198 \\ \mathrm{C} & 3.66663 & -1.54774 & 1.39831 \\ \mathrm{H} & 4.29611 & -2.00522 & 0.64894 \\ \mathrm{C} & 2.30081 & -1.38161 & 1.18920 \\ \mathrm{C} & 1.47376 & -0.82955 & 2.16407 \\ \mathrm{H} & 0.40873 & -0.72984 & 2.00887 \\ \mathrm{~S} & 1.55175 & -2.12537 & -0.25793 \\ \mathrm{~N} & 2.70545 & -2.07480 & -1.39518 \\ \mathrm{C} & 2.56483 & -3.09139 & -2.45550 \\ \mathrm{H} & 3.56529 & -3.34126 & -2.80727 \\ \mathrm{H} & 2.09339 & -3.98865 & -2.05899 \\ \mathrm{C} & 3.38986 & -0.84515 & -1.82102 \\ \mathrm{H} & 4.37944 & -1.13109 & -2.17778 \\ \mathrm{H} & 3.49916 & -0.15395 & -0.99247 \\ \mathrm{H} & 1.97215 & -2.70807 & -3.28860 \\ \mathrm{H} & 2.84110 & -0.36274 & -2.63215 \\ \mathrm{O} & -1.88207 & -2.79860 & -1.40385 \\ \mathrm{H} & -4.60718 & -2.87911 & -2.19611 \\ \mathrm{H} & -4.05076 & -1.18299 & -2.25594 \\ \mathrm{~S} & -2.32879 & -2.70801 & -2.81532 \\ \mathrm{C} & -4.01695 & -2.15200 & -2.75199 \\ \mathrm{H} & -0.61946 & -1.74513 & -0.82360 \\ \mathrm{~N} & 0.21387 & -1.18337 & -0.55665 \\ \mathrm{O} & -1.06866 & 0.56576 & -1.01880 \\ \mathrm{O} & -2.34031 & -4.01533 & -3.47593 \\ \mathrm{H} & -2.34816 & 2.07588 & -1.43674 \\ \mathrm{C} & 0.16866 & 0.19771 & -0.70703 \\ \mathrm{O} & -1.57756 & -1.68971 & -3.56158 \\ \mathrm{H} & -1.04600 & 2.52608 & -0.29912 \\ \mathrm{C} & -1.29019 & 1.98324 & -1.21047 \\ \mathrm{H} & -4.39419 & -2.06593 & -3.77029 \\ \mathrm{O} & 1.11346 & 0.93987 & -0.56247 \\ \mathrm{H} & -0.69105 & 2.34718 & -2.04336\end{array}$

\section{P2-MeOH}




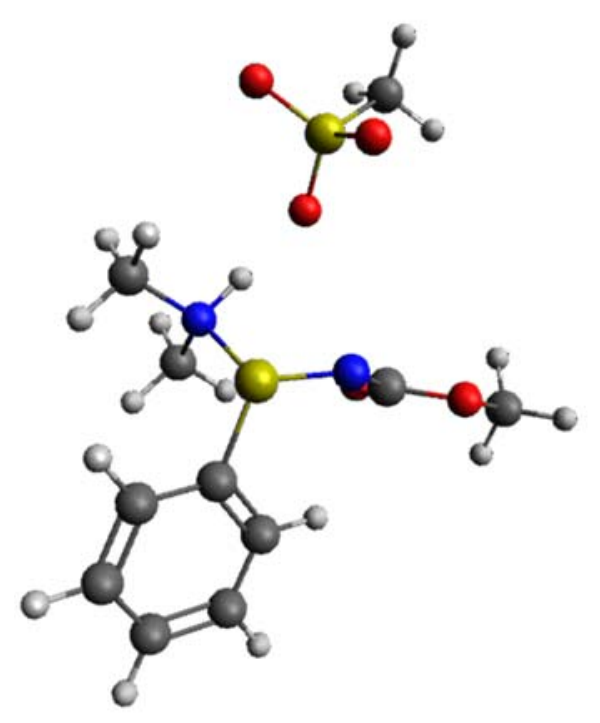

Energy $-46598.031 \mathrm{eV}$, i.e. $-1074575.848 \mathrm{Kcal} / \mathrm{mol}$; $\mathbf{E}_{\text {rel }}=-11.554 \mathrm{Kcal} / \mathrm{mol}$ Number of imaginary frequencies $=0$

\section{Coordinates $(x, y, z)$}

$\begin{array}{lrrr}\mathrm{H} & 3.33924 & 1.58318 & 2.67110 \\ \mathrm{C} & 3.33949 & 0.54863 & 2.35301 \\ \mathrm{C} & 4.41380 & -0.27999 & 2.66844 \\ \mathrm{H} & 5.24924 & 0.11568 & 3.23214 \\ \mathrm{C} & 4.41595 & -1.61340 & 2.27267 \\ \mathrm{H} & 5.24544 & -2.25961 & 2.52877 \\ \mathrm{C} & 3.34175 & -2.12670 & 1.55839 \\ \mathrm{H} & 3.33369 & -3.16751 & 1.25902 \\ \mathrm{C} & 2.28082 & -1.27855 & 1.23237 \\ \mathrm{C} & 2.26219 & 0.05651 & 1.63033 \\ \mathrm{H} & 1.43656 & 0.70819 & 1.38719 \\ \mathrm{~S} & 0.92567 & -2.08599 & 0.40476 \\ \mathrm{~N} & 1.61909 & -2.05272 & -1.44169 \\ \mathrm{C} & 2.13379 & -3.40925 & -1.75852 \\ \mathrm{H} & 3.02860 & -3.60840 & -1.17272 \\ \mathrm{H} & 1.36922 & -4.15283 & -1.54750 \\ \mathrm{C} & 2.58968 & -0.98520 & -1.77248 \\ \mathrm{H} & 3.53182 & -1.17446 & -1.26435 \\ \mathrm{H} & 2.18319 & -0.01906 & -1.49368 \\ \mathrm{H} & 2.38083 & -3.43195 & -2.81946 \\ \mathrm{H} & 2.74922 & -1.02042 & -2.85128 \\ \mathrm{~N} & -0.40456 & -1.29885 & 0.49053 \\ \mathrm{O} & -1.74207 & 0.44680 & 0.72091 \\ \mathrm{H} & -3.07556 & 1.94541 & 0.97373 \\ \mathrm{C} & -0.65480 & -0.00395 & 0.08854 \\ \mathrm{H} & -1.43776 & 2.50648 & 0.53819 \\ \mathrm{C} & -2.20794 & 1.76091 & 0.34630 \\ \mathrm{O} & -0.04575 & 0.62853 & -0.75572 \\ \mathrm{H} & -2.49296 & 1.77435 & -0.70483 \\ \mathrm{H} & 0.74316 & -1.90255 & -1.99526\end{array}$




$\begin{array}{llll}\mathrm{C} & -2.89138 & -2.03237 & -4.31913 \\ \mathrm{H} & -3.34815 & -1.19268 & -3.79703 \\ \mathrm{H} & -3.65059 & -2.76909 & -4.57838 \\ \mathrm{H} & -2.38297 & -1.68454 & -5.21719 \\ \mathrm{O} & -1.09969 & -3.90901 & -3.99607 \\ \mathrm{O} & -0.71524 & -1.74208 & -2.92884 \\ \mathrm{O} & -2.43646 & -3.25518 & -2.05587 \\ \mathrm{~S} & -1.70246 & -2.80073 & -3.24379\end{array}$

\section{$\mathrm{C} 1-\mathrm{MeOH}$}

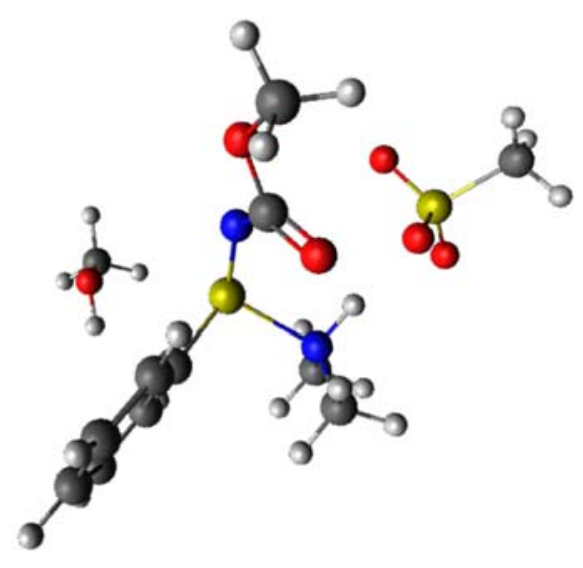

Energy $-49748.737 \mathrm{eV}$, i.e. $-1147232.836 \mathrm{Kcal} / \mathrm{mol}$

MeOH Energy $-3150.679 \mathrm{eV}$, i.e. $-72656.365 \mathrm{Kcal} / \mathrm{mol}$

Subtraction $-46598.058 \mathrm{eV}$, i.e. $-1074576.471 \mathrm{Kcal} / \mathrm{mol} ; \mathbf{E}_{\text {rel }}=10,931 \mathrm{Kcal} / \mathrm{mol}$

Number of imaginary frequencies $=0$

Coordinates $(x, y, z)$

$\begin{array}{lrrr}\mathrm{H} & 3.53427 & 1.72858 & 2.39928 \\ \mathrm{C} & 3.45335 & 0.68306 & 2.13160 \\ \mathrm{C} & 4.46824 & -0.20747 & 2.47510 \\ \mathrm{H} & 5.33834 & 0.15170 & 3.00999 \\ \mathrm{C} & 4.36489 & -1.55433 & 2.14535 \\ \mathrm{H} & 5.14614 & -2.24957 & 2.42400 \\ \mathrm{C} & 3.24513 & -2.01628 & 1.46623 \\ \mathrm{H} & 3.14839 & -3.06795 & 1.22569 \\ \mathrm{C} & 2.24589 & -1.10833 & 1.10824 \\ \mathrm{C} & 2.33178 & 0.24102 & 1.44452 \\ \mathrm{H} & 1.55022 & 0.93945 & 1.18539 \\ \mathrm{~S} & 0.83285 & -1.85892 & 0.31910 \\ \mathrm{~N} & 1.48384 & -1.86525 & -1.53875 \\ \mathrm{C} & 2.01125 & -3.22196 & -1.82866 \\ \mathrm{H} & 2.92960 & -3.38040 & -1.26685 \\ \mathrm{H} & 1.26998 & -3.97212 & -1.56500 \\ \mathrm{C} & 2.43705 & -0.79976 & -1.92244 \\ \mathrm{H} & 3.38722 & -0.96150 & -1.41922\end{array}$




$\begin{array}{rrrr}\mathrm{H} & 2.02244 & 0.17087 & -1.67434 \\ \mathrm{H} & 2.22373 & -3.27693 & -2.89573 \\ \mathrm{H} & 2.58550 & -0.87323 & -3.00099 \\ \mathrm{~N} & -0.46150 & -1.01822 & 0.43001 \\ \mathrm{O} & -1.72717 & 0.76901 & 0.72420 \\ \mathrm{H} & -2.99540 & 2.31115 & 1.04344 \\ \mathrm{C} & -0.68047 & 0.28788 & 0.04667 \\ \mathrm{H} & -1.36155 & 2.81860 & 0.53618 \\ \mathrm{C} & -2.16428 & 2.10016 & 0.37626 \\ \mathrm{O} & -0.08258 & 0.90836 & -0.81483 \\ \mathrm{H} & -2.49512 & 2.12880 & -0.66112 \\ \mathrm{H} & 0.58886 & -1.73382 & -2.06762 \\ \mathrm{C} & -2.72581 & -2.00609 & -4.76495 \\ \mathrm{H} & -3.36253 & -1.25589 & -4.29839 \\ \mathrm{H} & -3.33362 & -2.80954 & -5.17907 \\ \mathrm{H} & -2.12216 & -1.55095 & -5.54900 \\ \mathrm{O} & -0.79917 & -3.68340 & -4.21060 \\ \mathrm{O} & -0.86023 & -1.54406 & -3.01668 \\ \mathrm{O} & -2.50008 & -3.29157 & -2.50124 \\ \mathrm{~S} & -1.64470 & -2.69364 & -3.53260 \\ \mathrm{O} & 1.06231 & -4.44330 & 3.11173 \\ \mathrm{C} & 0.61520 & -5.80347 & 3.12032 \\ \mathrm{H} & 1.19392 & -6.40837 & 3.82478 \\ \mathrm{H} & 0.68090 & -6.25219 & 2.12487 \\ \mathrm{H} & -0.42774 & -5.79716 & 3.43617 \\ \mathrm{H} & 1.98243 & -4.42864 & 2.82456\end{array}$

\section{$\mathrm{C} 2-\mathrm{MeOH}$}

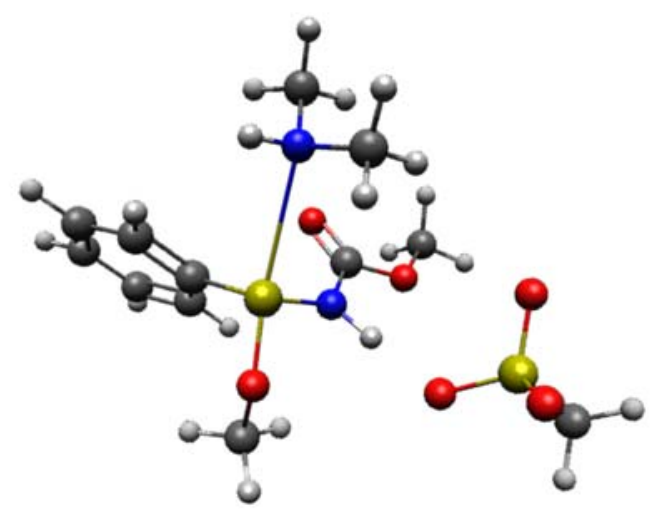

Energy $-49748.655 \mathrm{eV}$, i.e. $-1147230.945 \mathrm{Kcal} / \mathrm{mol}$

MeOH Energy $-3150.679 \mathrm{eV}$, i.e. $-72656.365 \mathrm{Kcal} / \mathrm{mol}$

Subtraction $-46597.976 \mathrm{eV}$, i.e. $-1074574.580 \mathrm{Kcal} / \mathrm{mol}$; $\mathbf{E}_{\mathrm{rel}}=12.822 \mathrm{Kcal} / \mathrm{mol}$ Number of imaginary frequencies $=0$

Coordinates $(x, y, z)$

$\begin{array}{rrrr}\mathrm{H} & 4.177578 & 0.863351 & 7.811358 \\ \mathrm{C} & 5.044740 & -0.339223 & 6.402493 \\ \mathrm{C} & 7.627584 & -0.844109 & 6.501102\end{array}$ 

H $\quad 8.761828 \quad-0.025497 \quad 7.994513$
C $\quad 8.757037 \quad-2.388149 \quad 4.699220$
H $\quad 10.763932 \quad-2.773054 \quad 4.776518$
C $\quad 7.307279-3.437326 \quad 2.779845$
H $\quad 8.174466-4.639176 \quad 1.368890$
C $\quad 4.726066-2.908697 \quad 2.703010$
C $\quad 3.566149-1.368392 \quad 4.500234$
H $\quad 1.566115-0.9643114 .418188$
S $\quad 3.070083-4.578768 \quad 0.324870$
N $\quad 5.979854-2.497194-3.620055$
C $\quad 4.796068-3.714825-5.793480$
H $\quad 6.047746-3.802132-7.448658$
H $\quad 4.243813-5.643709-5.314692$
C $\quad 6.897690 \quad 0.041705-4.199102$
H $\quad 8.267182 \quad 0.054758-5.761307$
H $\quad 7.804565 \quad 0.856611-2.536859$
H $\quad 3.097751-2.674549-6.335204$
H $\quad 5.317141 \quad 1.253004-4.733225$
$\begin{array}{llll}\text { O } & -3.197810 & -5.658527 & -2.486055\end{array}$
H $\quad-8.229464-6.570873-4.063934$
H $\quad-7.566175-3.292935-4.516857$
S $\quad-3.958620-5.859928-5.173845$
C $\quad-7.251475-5.193773-5.239860$
H $\quad-0.860500 \quad-3.798758-1.190598$
N $\quad 0.627479-2.732179-0.457169$
$\begin{array}{lllll}\text { O } & -1.587009 & 0.459831 & -1.961334\end{array}$
$\begin{array}{llll}0 & -3.656830 & -8.440919 & -6.137977\end{array}$
H $\quad-3.843462 \quad 3.213257 \quad-3.342632$
C $\quad 0.622461-0.157089-0.913209$
$\begin{array}{llll}\mathrm{O} & -2.722190 & -3.941550 & -6.749825\end{array}$
H $\quad-1.898723 \quad 4.212233-0.773812$
$\begin{array}{llll}\text { C } & -1.971074 & 3.108227 & -2.508677\end{array}$
$\mathrm{H} \quad-7.909427 \quad-5.331241-7.185419$
$\begin{array}{lllll}\text { O } & 2.298391 & 1.314341 & -0.413420\end{array}$
$\begin{array}{llll}\mathrm{H} & -0.553117 & 3.771615 & -3.843389\end{array}$
H $\quad 7.465280-3.563263-3.040375$
$\begin{array}{llll}\text { O } & 1.548897 & -6.772817 & 1.780842\end{array}$
$\begin{array}{llll}\text { C } & -0.074870 & -6.383973 & 3.976825\end{array}$
H $\quad-1.073292-8.167585 \quad 4.178736$
H $\quad-1.411030-4.861170 \quad 3.633722$
H $\quad 1.080764 \quad-6.014543 \quad 5.635909$

\section{P3-MeOH}

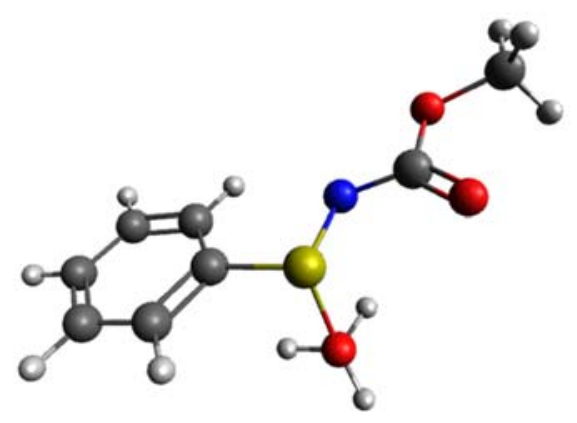


Energy $-27985.626 \mathrm{eV}$, i.e. $-645363.702 \mathrm{Kcal} / \mathrm{mol}$

MeOH Energy $-3150.679 \mathrm{eV}$, i.e. $-72656.365 \mathrm{Kcal} / \mathrm{mol}$

Dimethylammonium Cation Energy $-3692.542 \mathrm{eV}$, i.e. $85152.020 \mathrm{Kcal} / \mathrm{mol}$

Mesylate Anion Energy $-18071.227 \mathrm{eV}$, i.e. $-416732.288 \mathrm{Kcal} / \mathrm{mol}$

Total (Energy- MeOH + Dimethylammonium Cation + Mesylate Anion) $-46598.716 \mathrm{eV}$, i.e. $-1074591.644 \mathrm{Kcal} / \mathrm{mol} ; \mathbf{E}_{\text {rel }}=-4.242 \mathrm{Kcal} / \mathrm{mol}$

Number of imaginary frequencies $=0$

Coordinates $(x, y, z)$

$\begin{array}{lrrr}\mathrm{H} & 2.26723 & -0.01687 & 4.86478 \\ \mathrm{C} & 2.76797 & -0.37970 & 3.97608 \\ \mathrm{C} & 4.06622 & -0.87442 & 4.05606 \\ \mathrm{H} & 4.57902 & -0.89408 & 5.00967 \\ \mathrm{C} & 4.71255 & -1.33687 & 2.91316 \\ \mathrm{H} & 5.72484 & -1.71561 & 2.97299 \\ \mathrm{C} & 4.06135 & -1.30701 & 1.68715 \\ \mathrm{H} & 4.56362 & -1.66409 & 0.79586 \\ \mathrm{C} & 2.75742 & -0.81766 & 1.62310 \\ \mathrm{C} & 2.10348 & -0.34530 & 2.75555 \\ \mathrm{H} & 1.09837 & 0.04304 & 2.68138 \\ \mathrm{~S} & 2.02041 & -0.82026 & -0.00322 \\ \mathrm{~N} & 0.64048 & -0.07311 & 0.27764 \\ \mathrm{O} & -1.04702 & 1.11614 & -0.53105 \\ \mathrm{H} & -2.63740 & 2.19836 & -1.15083 \\ \mathrm{C} & -0.01327 & 0.31522 & -0.85872 \\ \mathrm{H} & -1.26622 & 2.22179 & -2.29054 \\ \mathrm{C} & -1.85746 & 1.60162 & -1.61704 \\ \mathrm{O} & 0.23577 & 0.00950 & -2.02243 \\ \mathrm{H} & -2.30044 & 0.77198 & -2.16719 \\ \mathrm{O} & 1.76373 & -2.42148 & -0.24716 \\ \mathrm{C} & 0.88970 & -3.15313 & 0.65007 \\ \mathrm{H} & 0.82428 & -4.15192 & 0.22486 \\ \mathrm{H} & -0.09856 & -2.69634 & 0.67756 \\ \mathrm{H} & 1.31909 & -3.20213 & 1.65043\end{array}$


Copies of ${ }^{1} \mathrm{H}$ and ${ }^{13} \mathrm{C}$ NMR spectra 


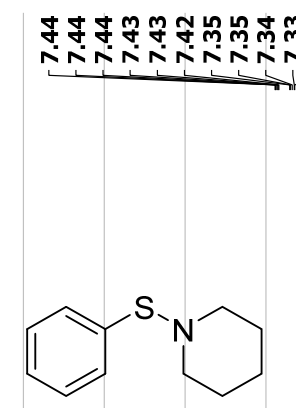

$1 a$

${ }^{1} \mathrm{H} \mathrm{NMR,}, 500 \mathrm{MHz}, \mathrm{CDCl}_{3}$

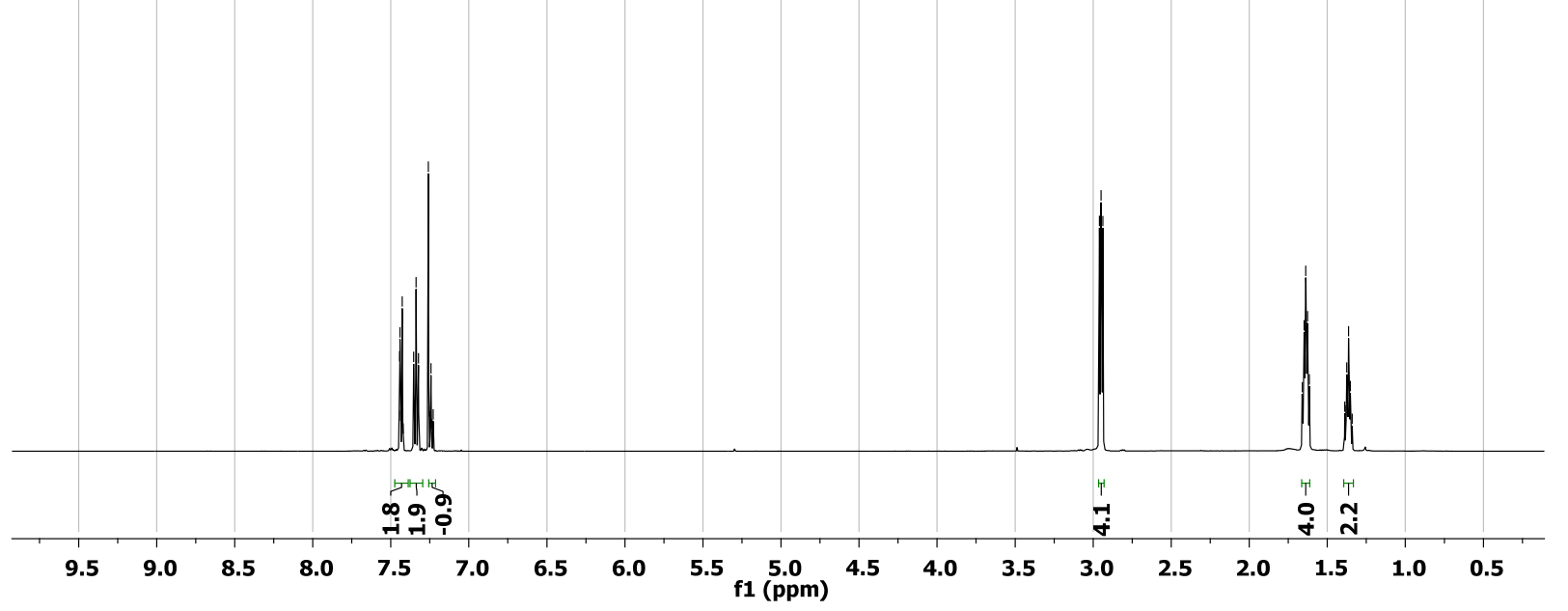

$1 b$

${ }^{1} \mathrm{H} \mathrm{NMR}, 300 \mathrm{MHz}, \mathrm{CDCl}_{3}$

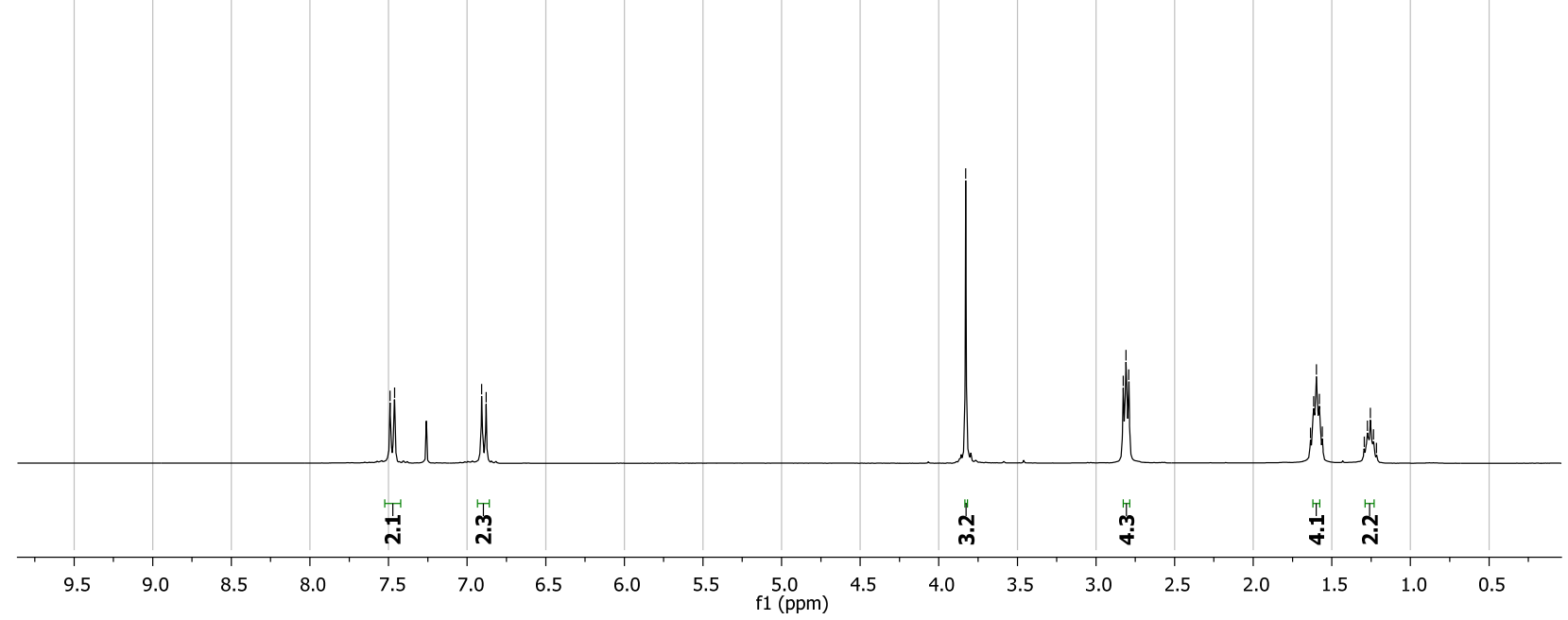




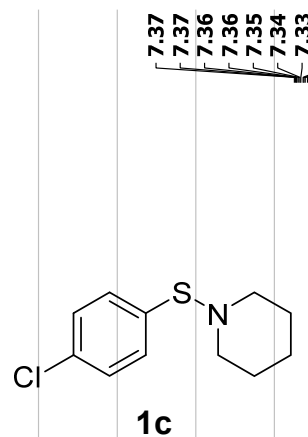

${ }^{1} \mathrm{H}$ NMR, $300 \mathrm{MHz}, \mathrm{CDCl}_{3}$
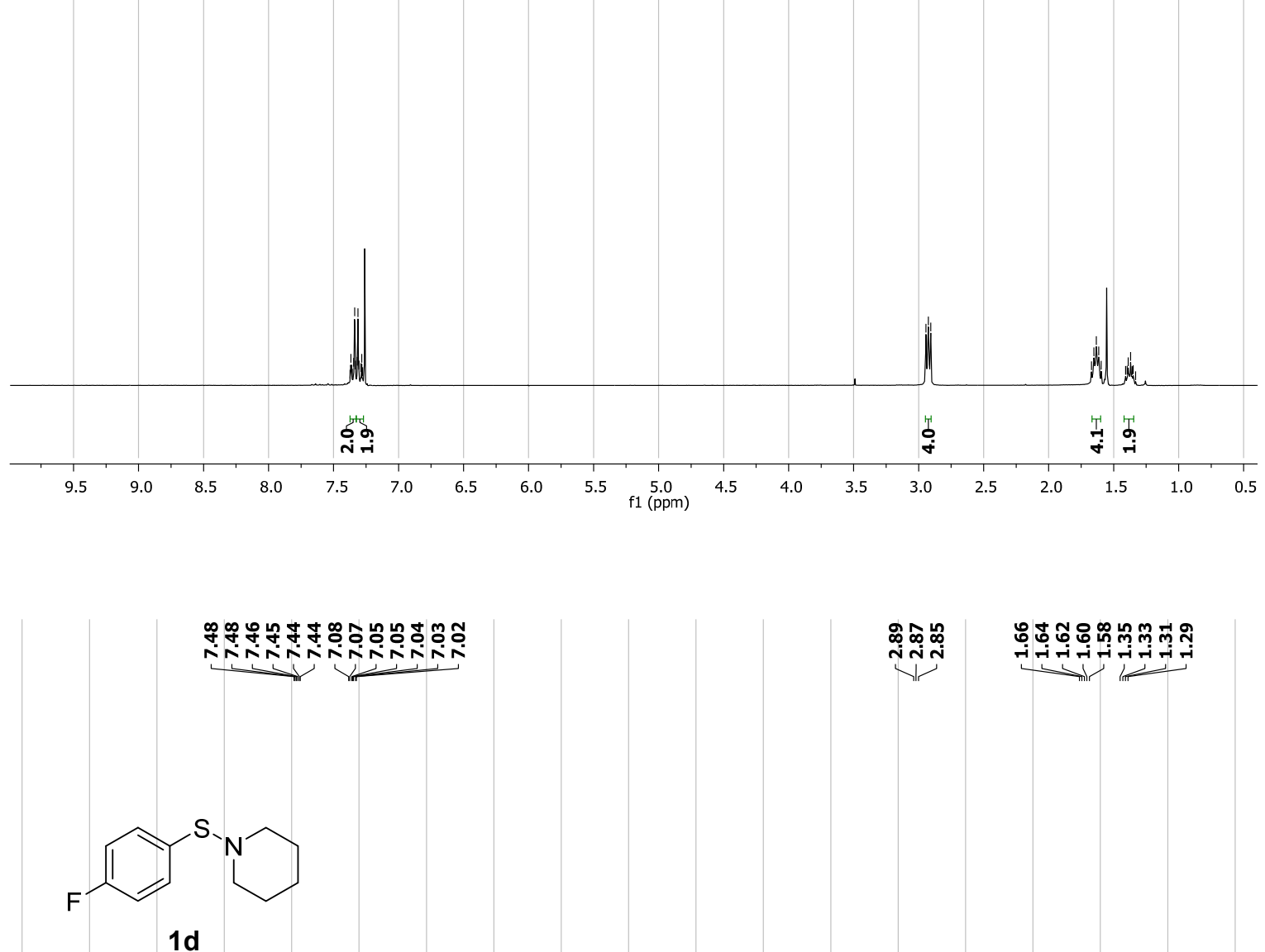

${ }^{1} \mathrm{H} \mathrm{NMR}, 300 \mathrm{MHz}, \mathrm{CDCl}_{3}$

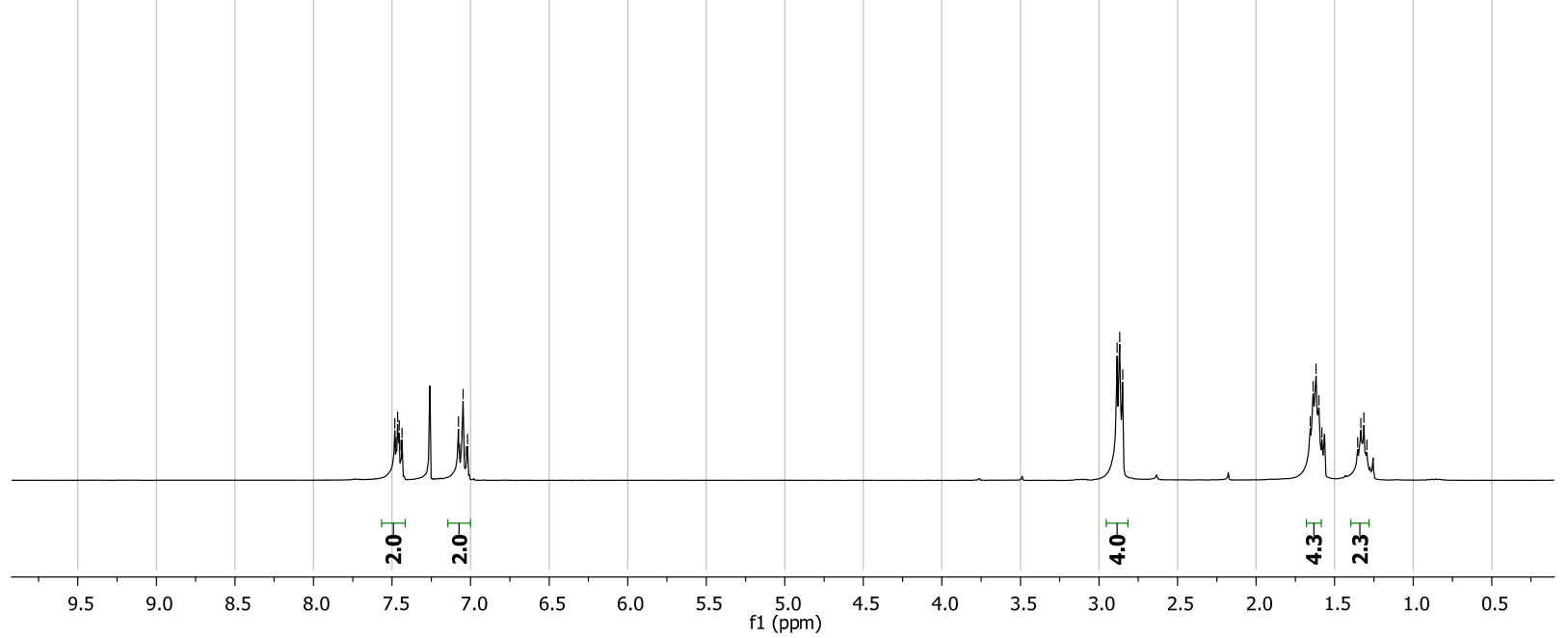



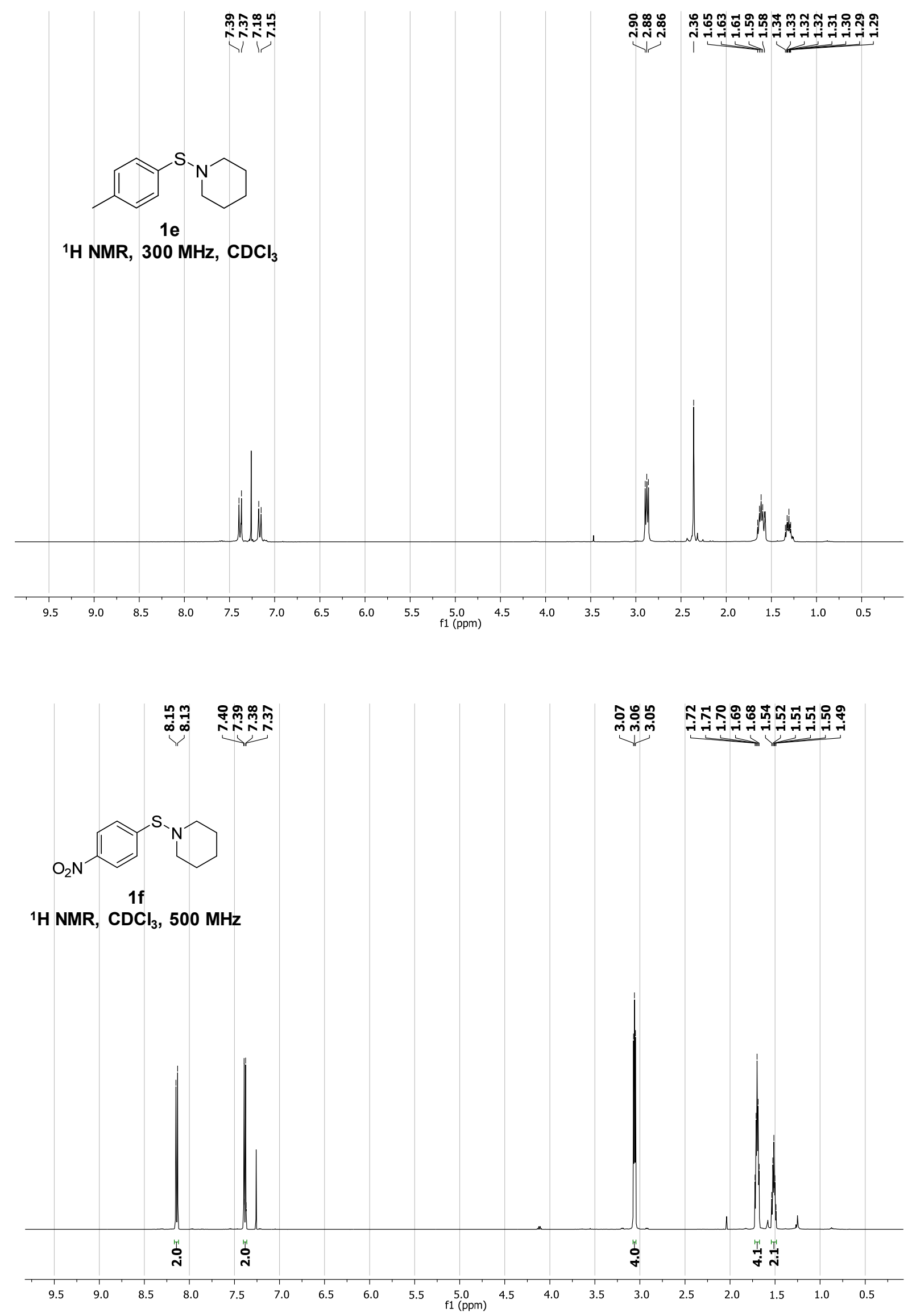

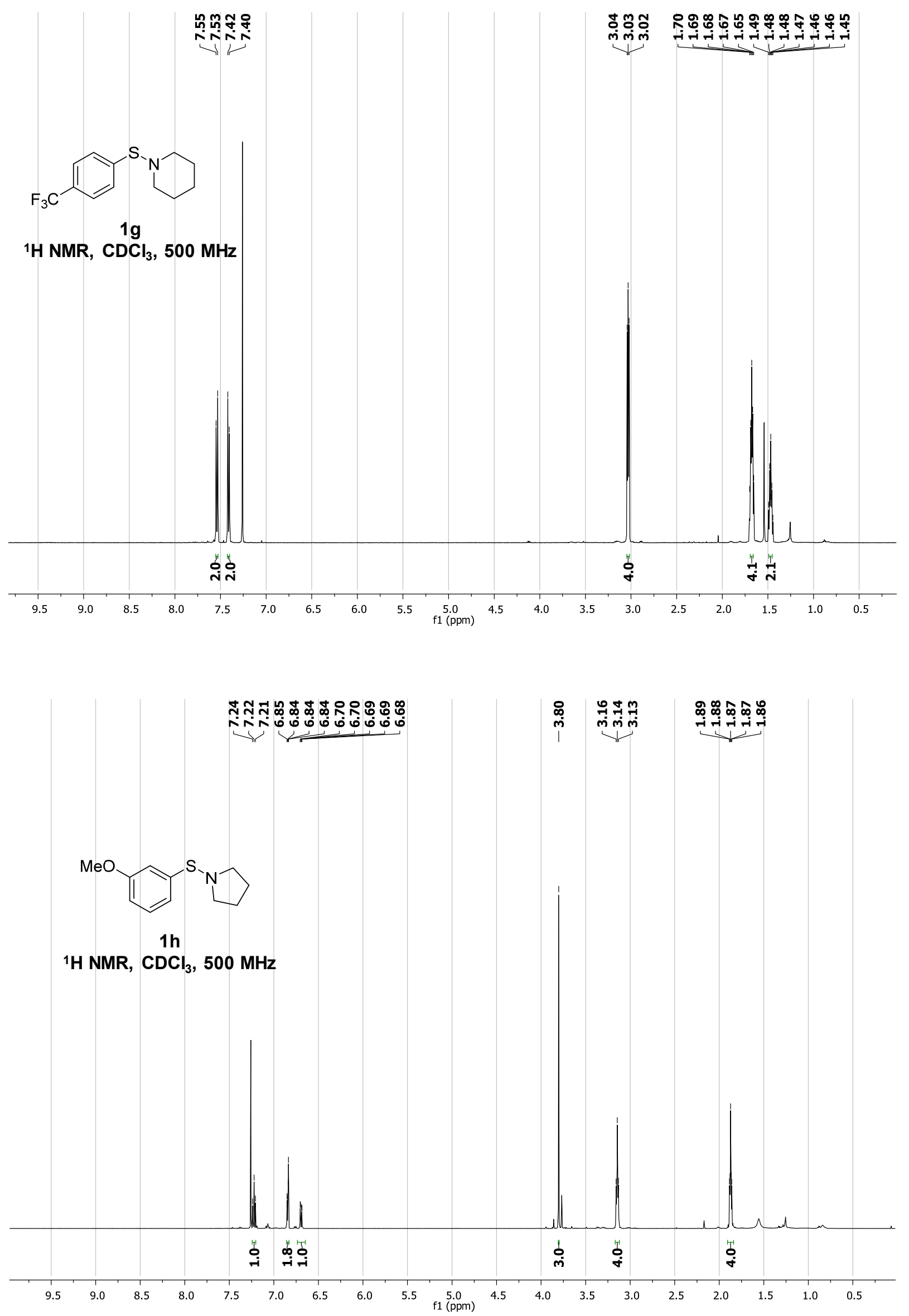

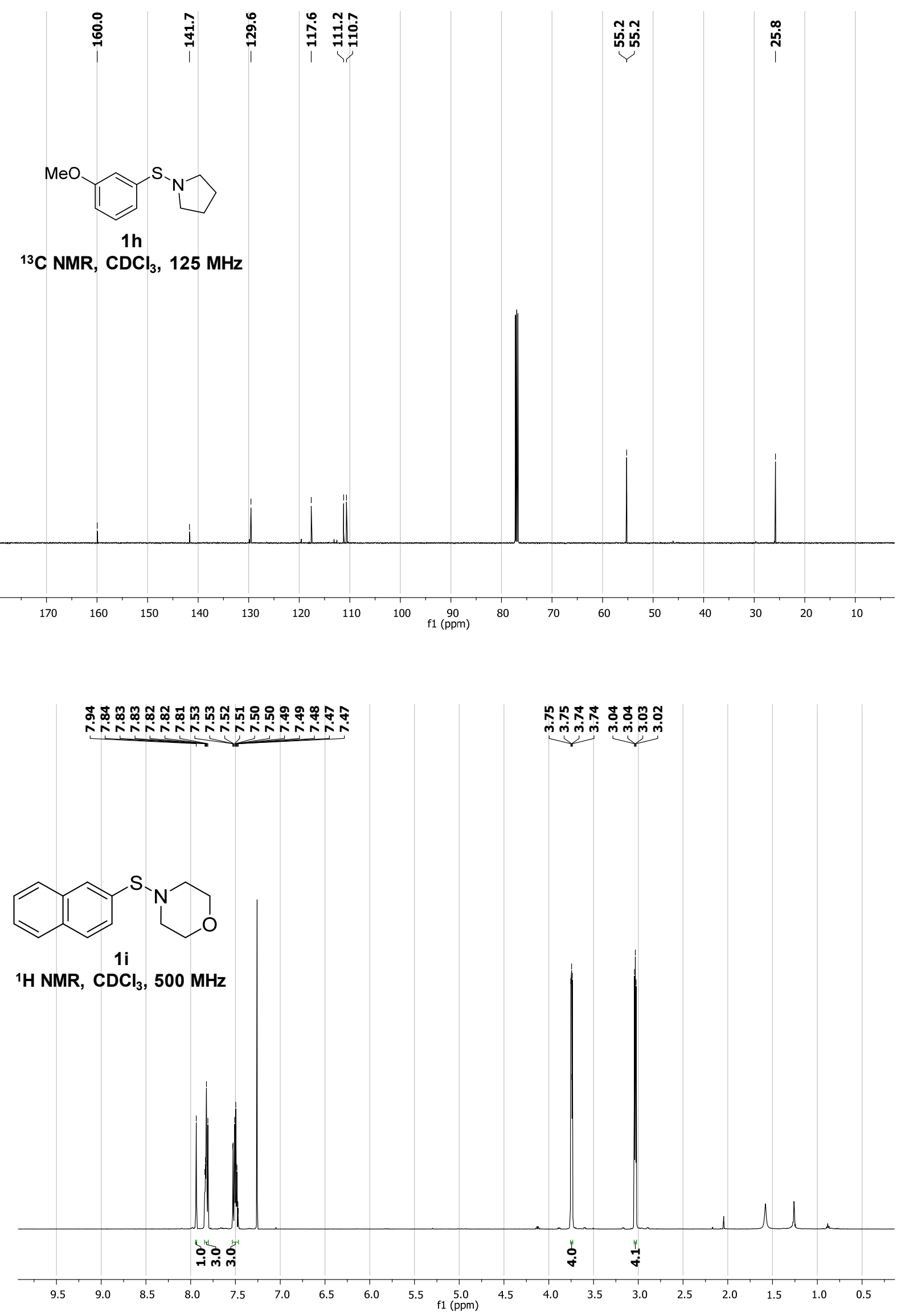

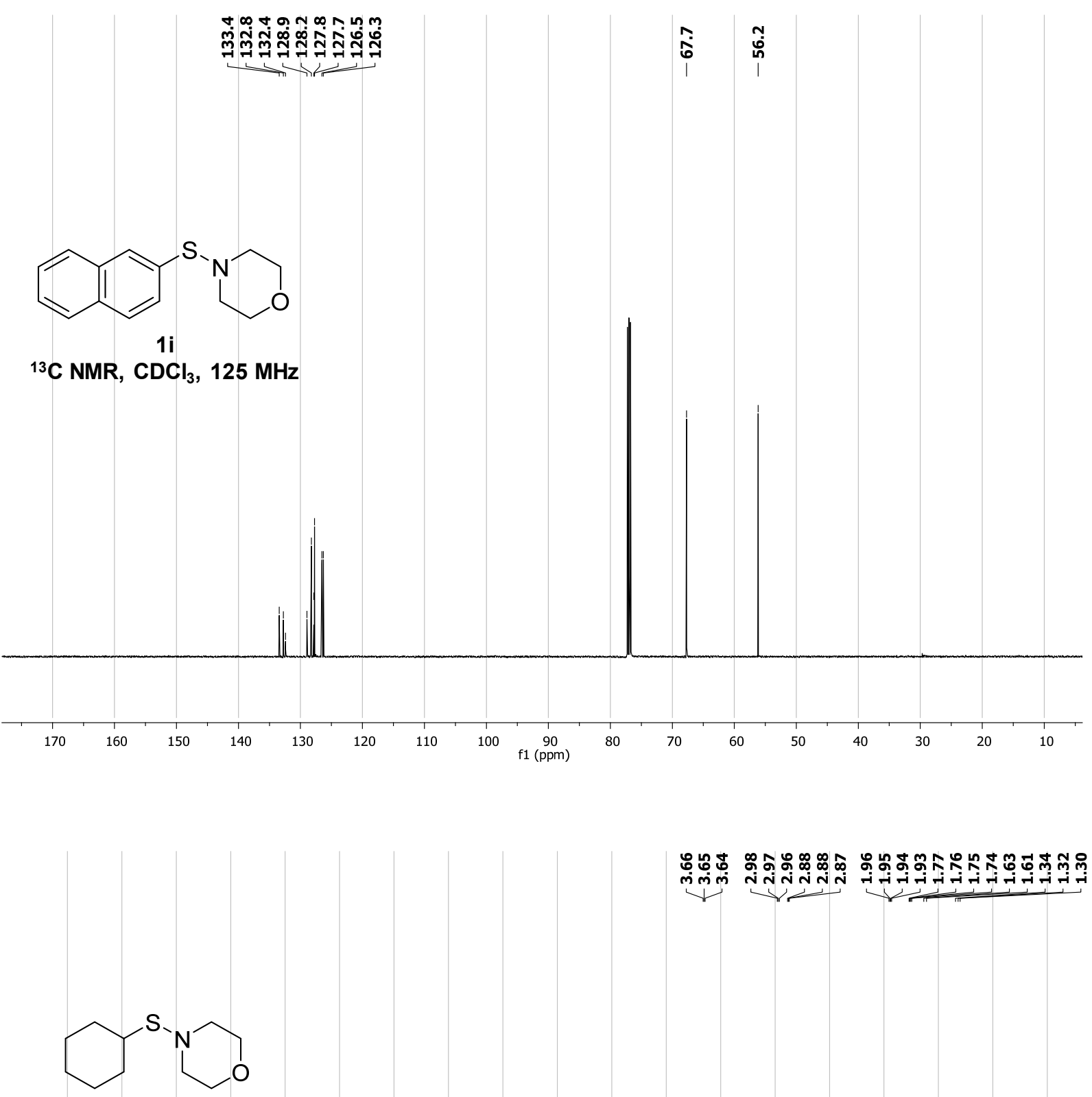

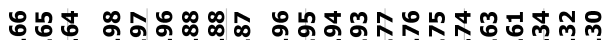

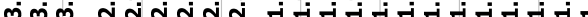

政

$1 \mathrm{j}$

${ }^{1} \mathrm{H} \mathrm{NMR}, \mathrm{CDCl}_{3}, 500 \mathrm{MHz}$

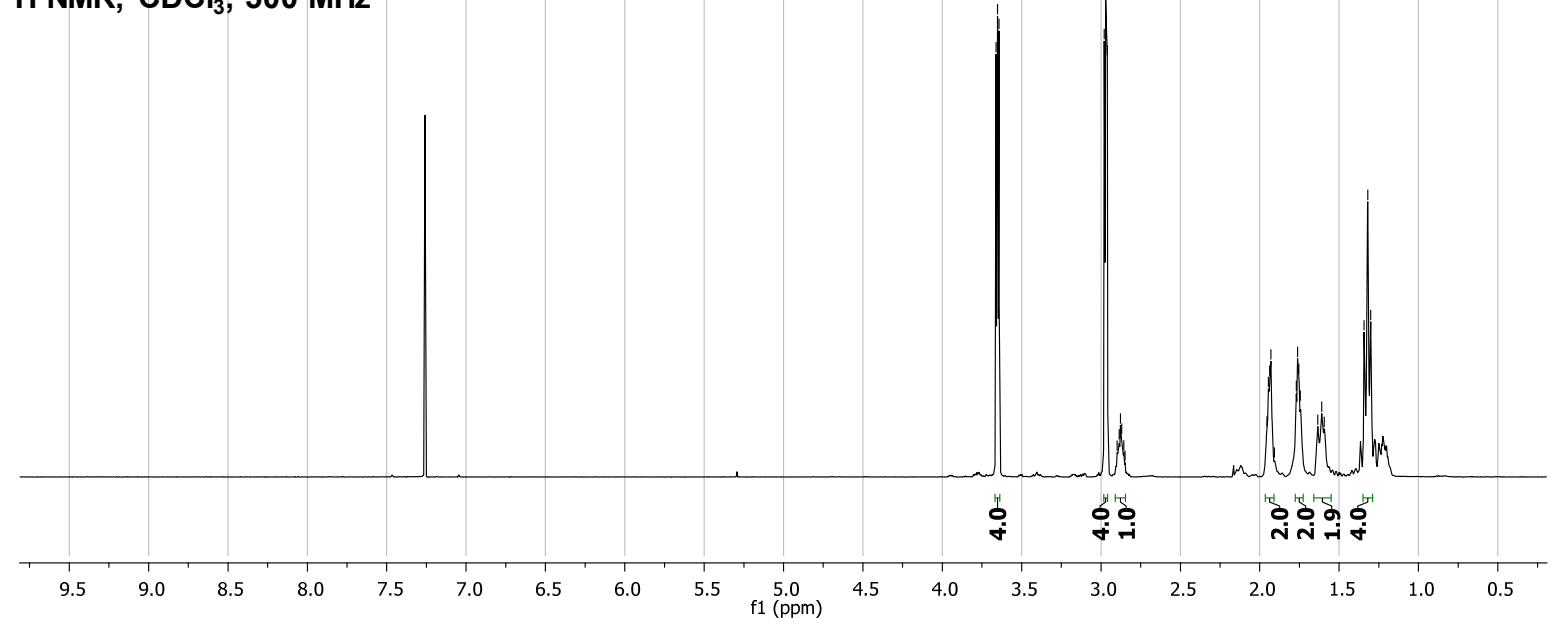




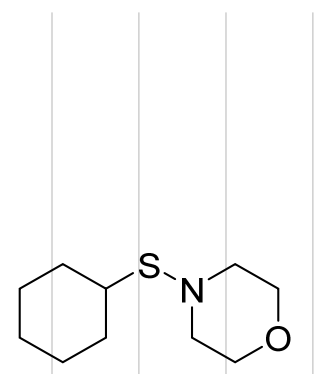

$1 \mathrm{j}$

${ }^{13} \mathrm{C} \mathrm{NMR,} \mathrm{CDCl}_{3}, 125 \mathrm{MHz}$

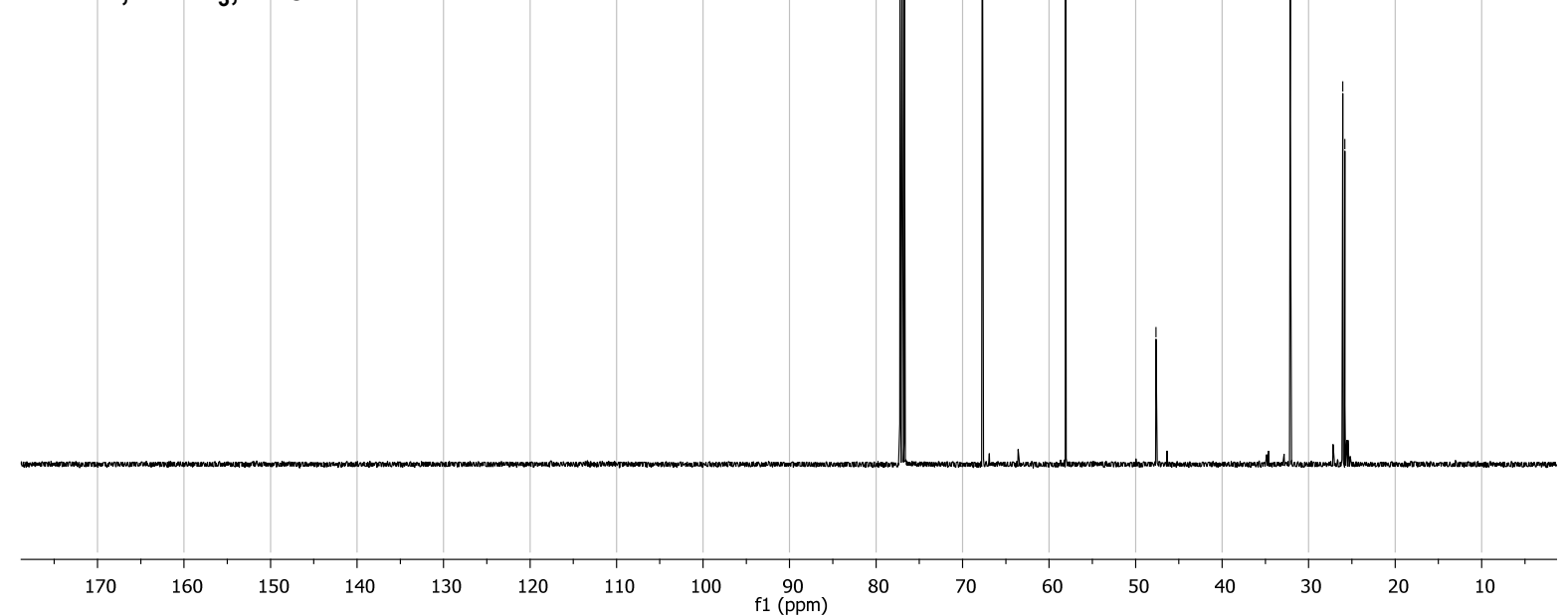

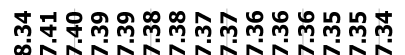

เุ

$\stackrel{m}{m}$

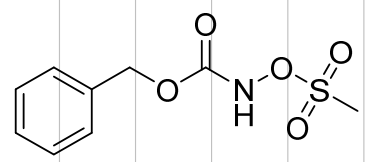

2a

${ }^{1} \mathrm{H} \mathrm{NMR}, \mathrm{CDCl}_{3}, 500 \mathrm{MHz}$

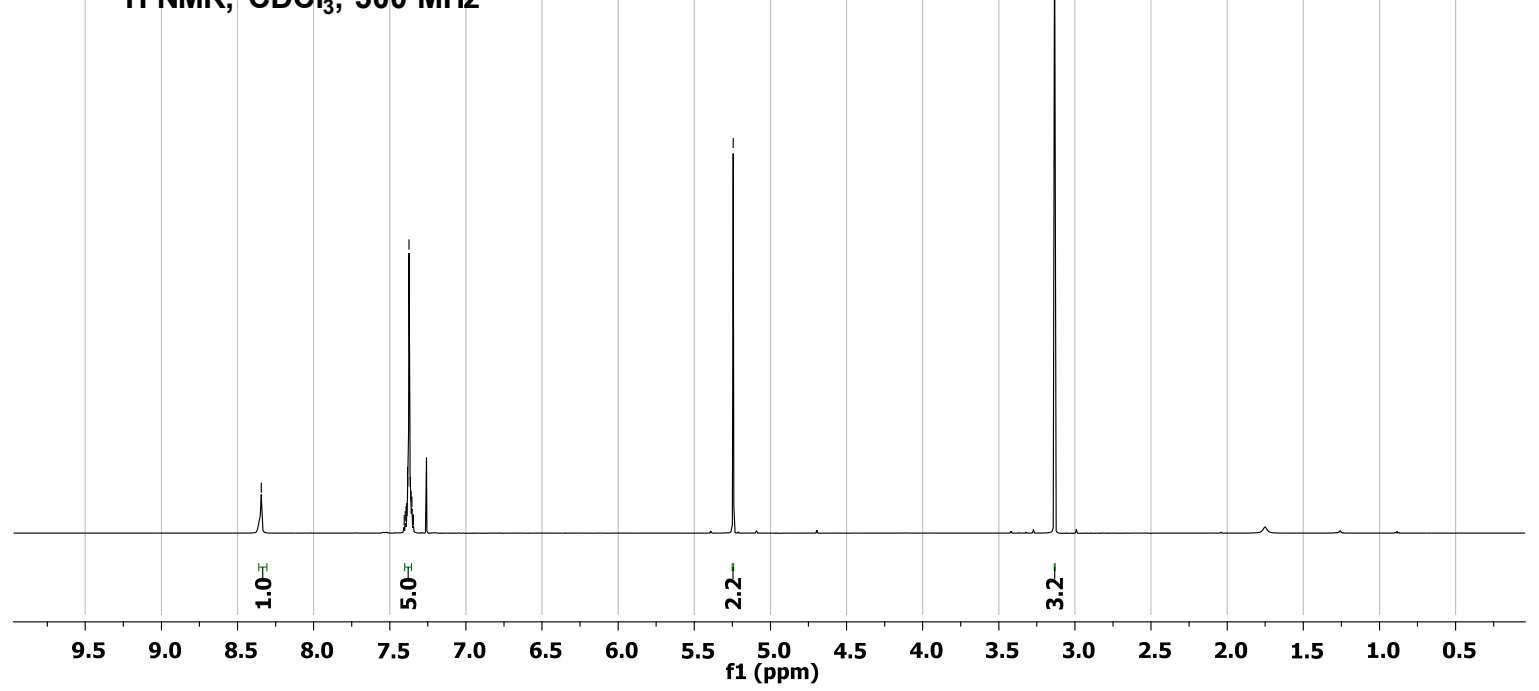



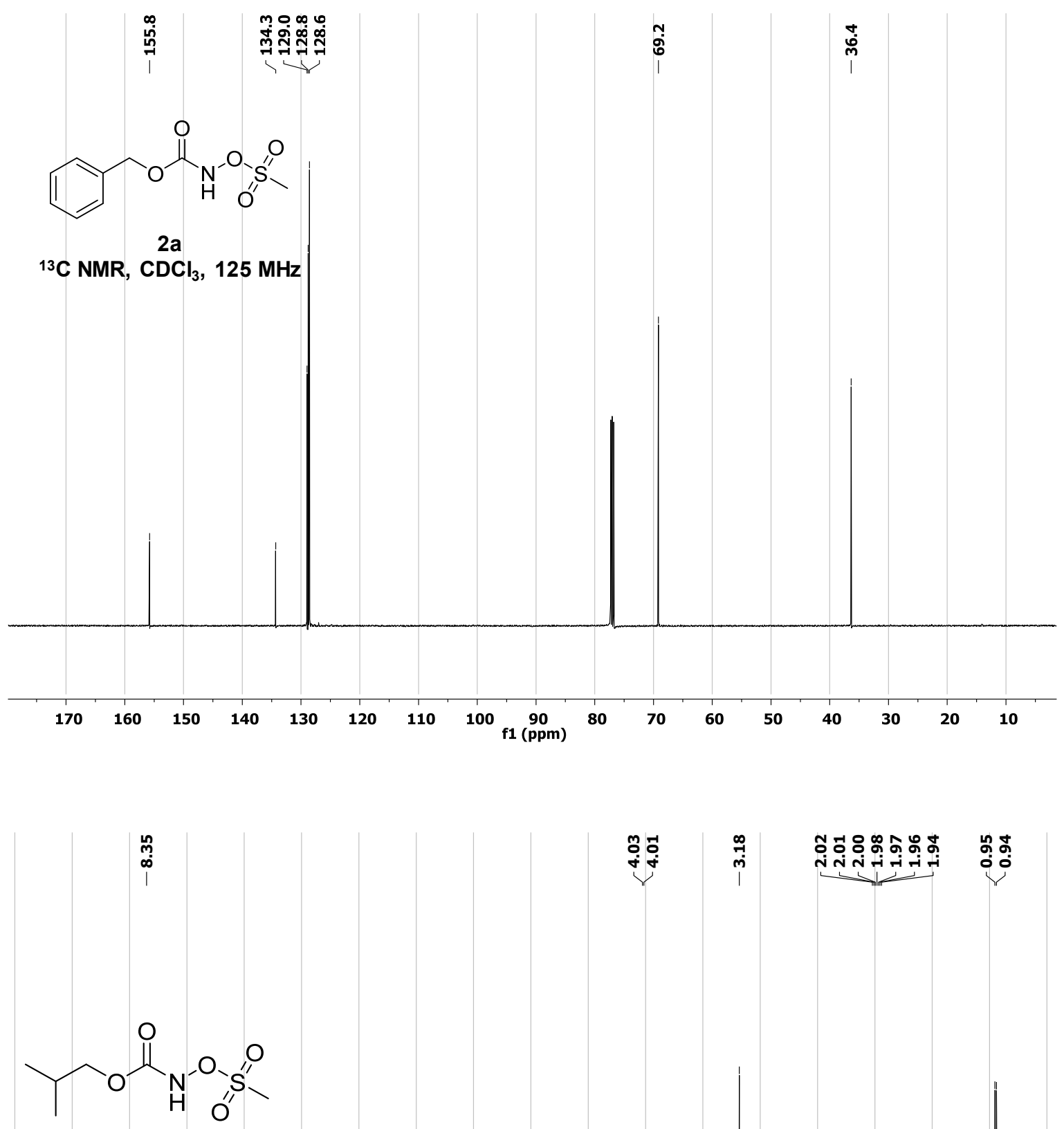

$2 b$

${ }^{1} \mathrm{H}$ NMR, $\mathrm{CDCl}_{3}, 500 \mathrm{MHz}$

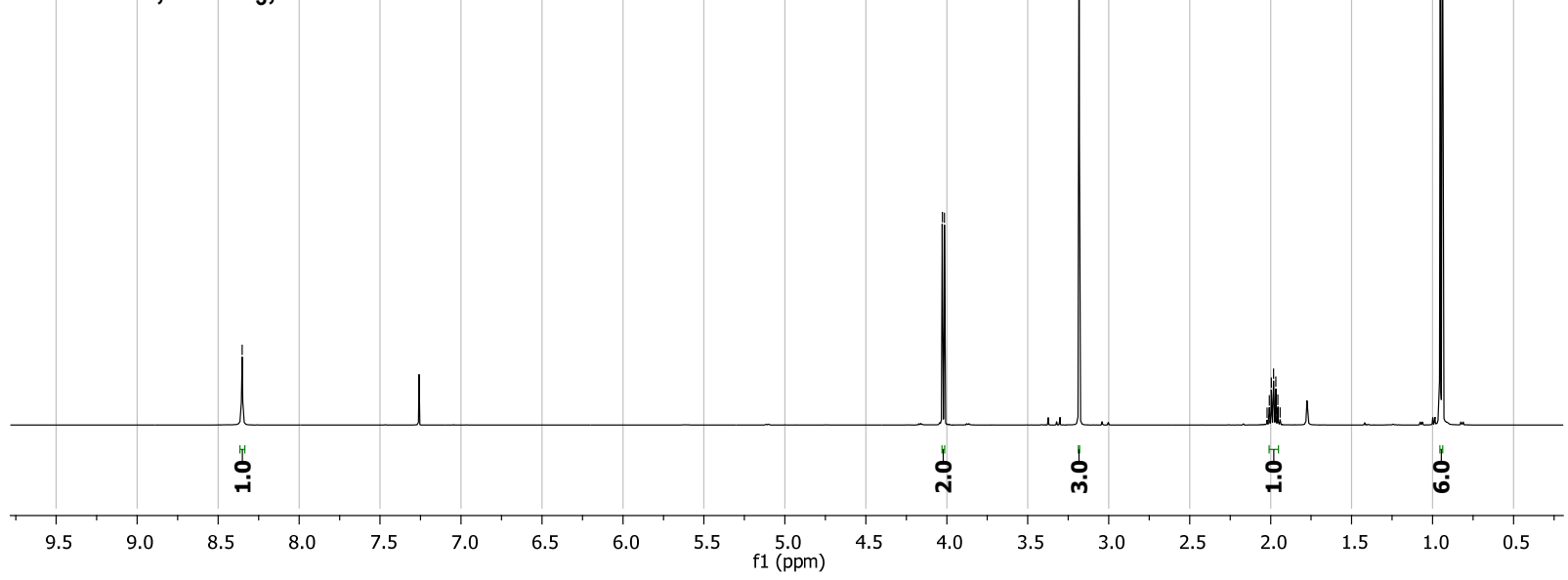




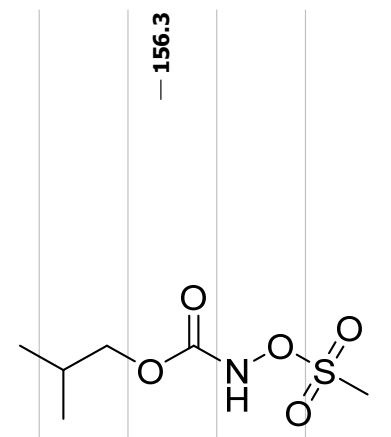

2b

${ }^{13} \mathrm{C} \mathrm{NMR,} \mathrm{CDCl}_{3}, 125 \mathrm{MHz}$

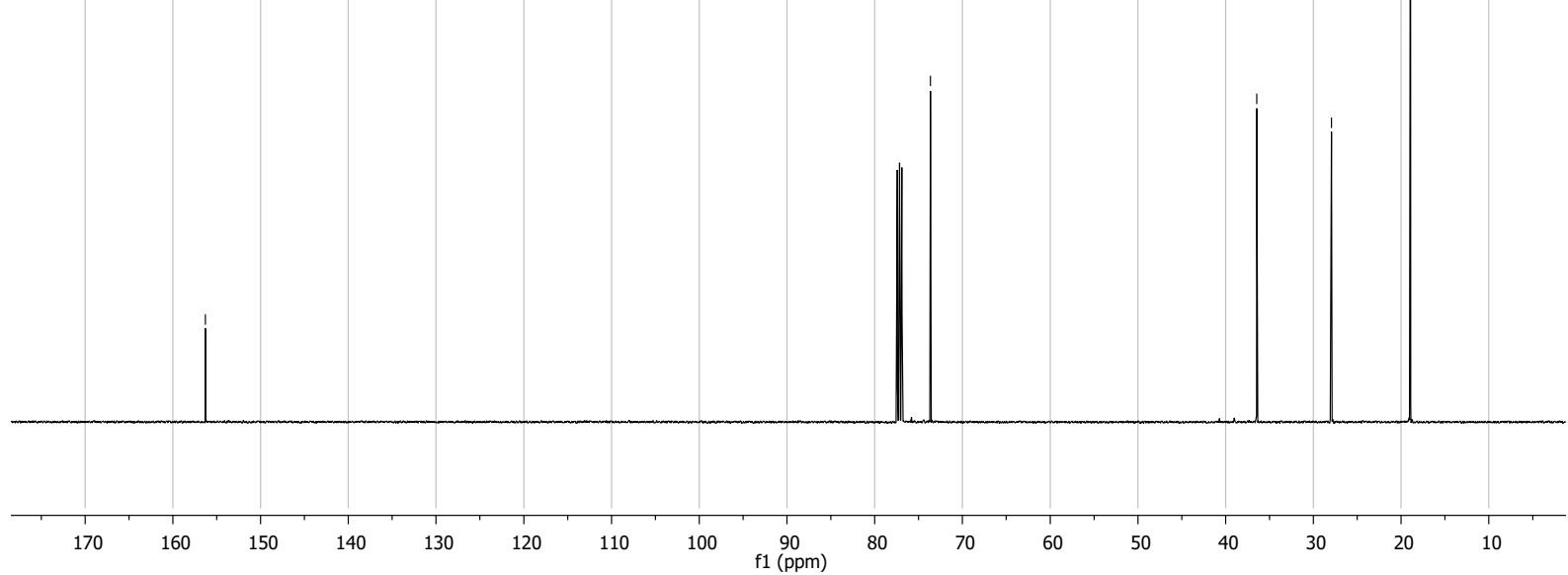

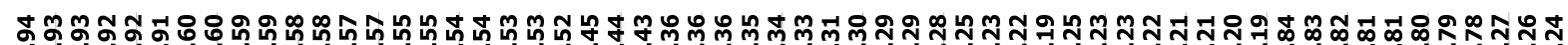

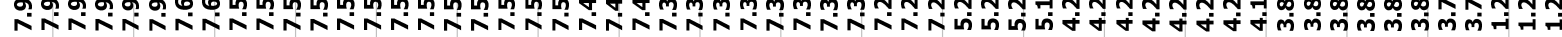<smiles>CCOS(=NC(=O)OCc1ccccc1)c1ccccc1</smiles>

${ }^{1} \mathrm{H} \mathrm{NMR}, \mathrm{CDCl}_{3}, 500 \mathrm{MHz}$

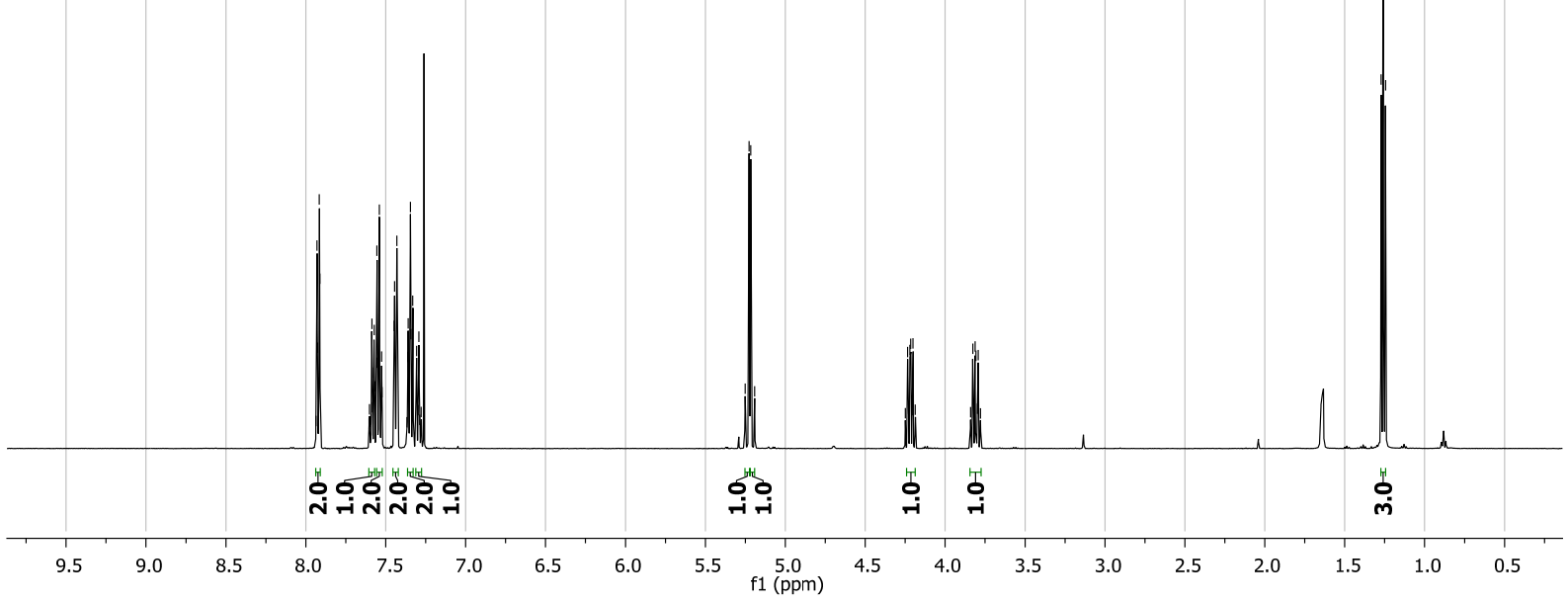



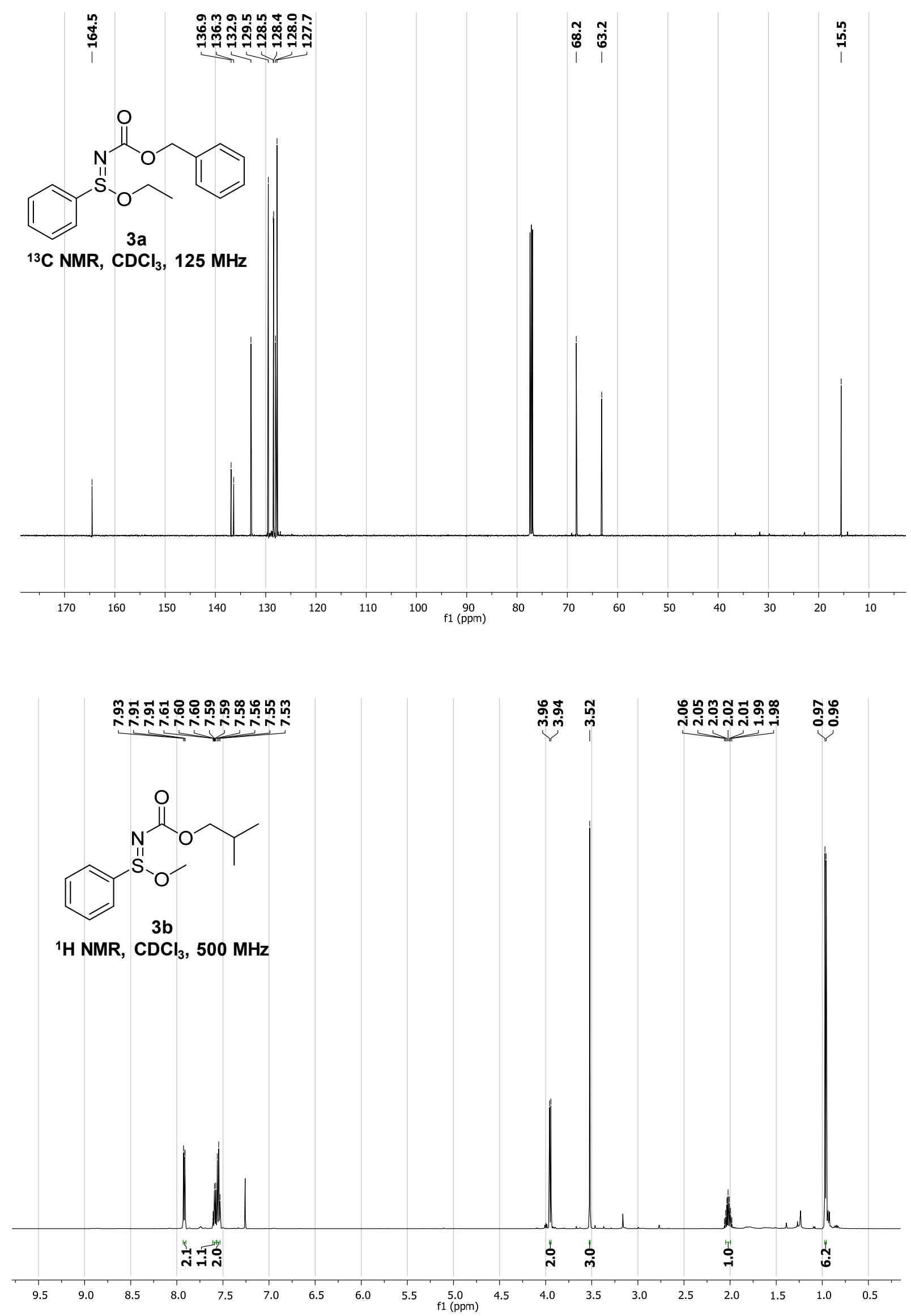


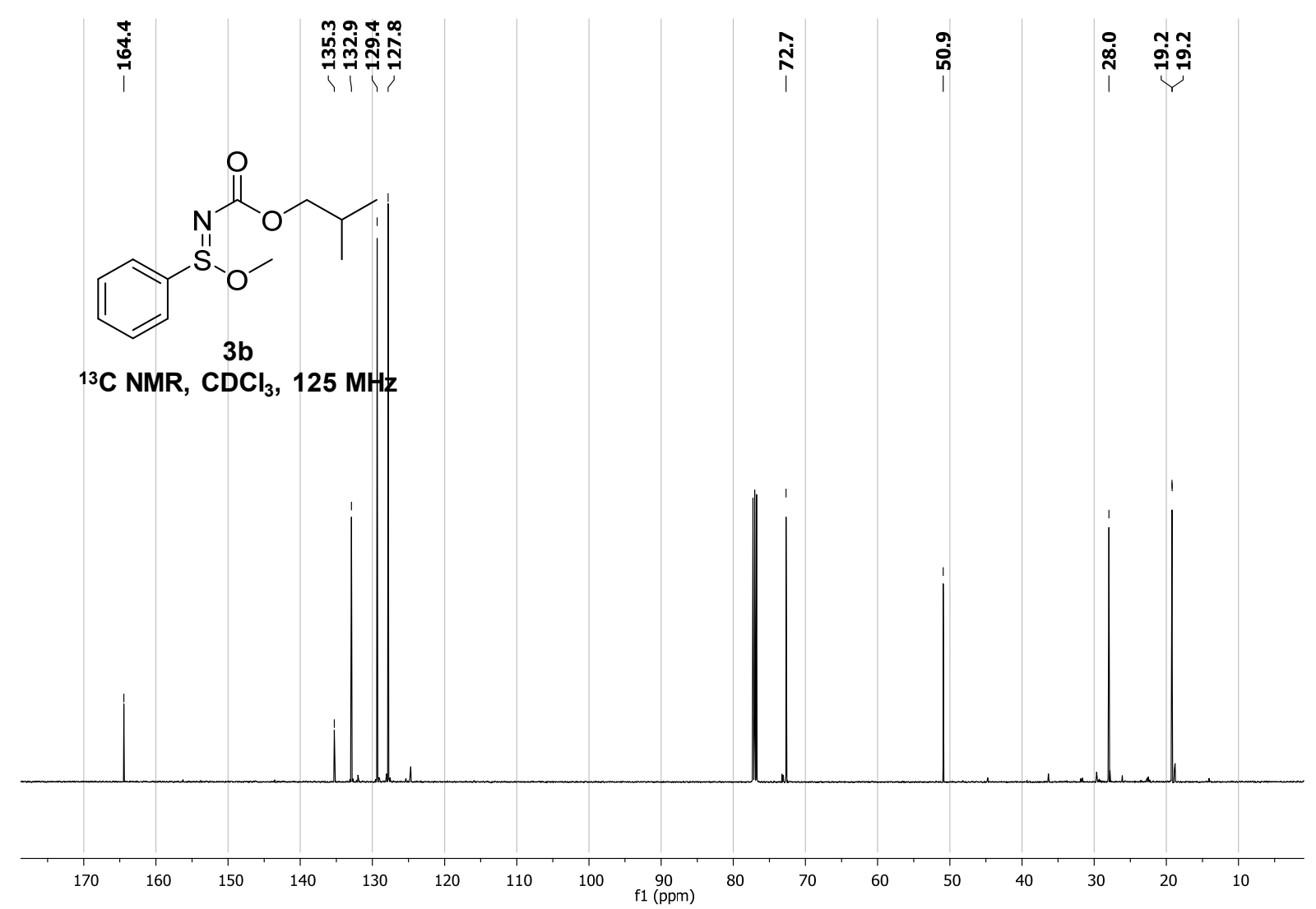

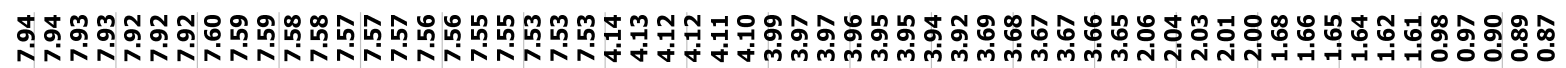<smiles>CCCOS(=NC(=O)OCC(C)C)c1ccccc1</smiles>

$3 c$

${ }^{1} \mathrm{H} \mathrm{NMR}, \mathrm{CDCl}_{3}, 500 \mathrm{MHz}$

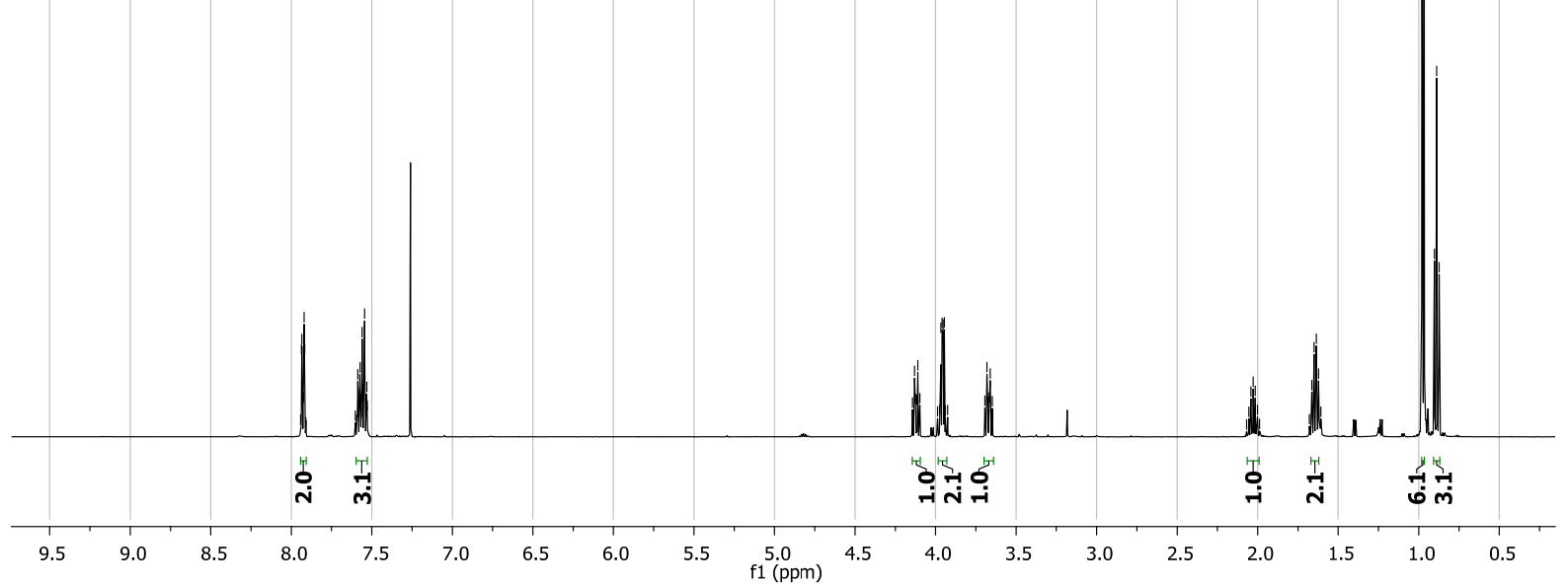




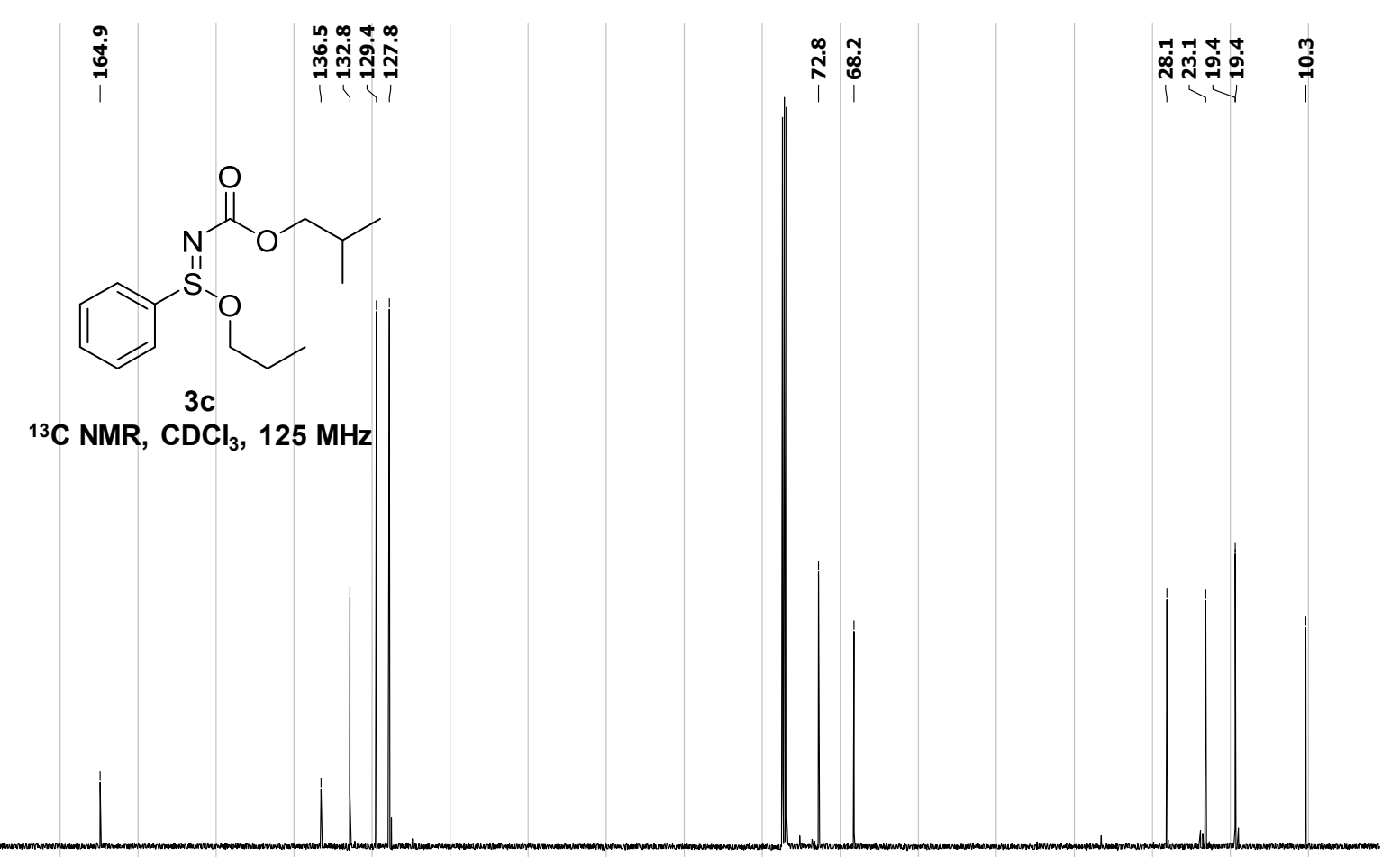

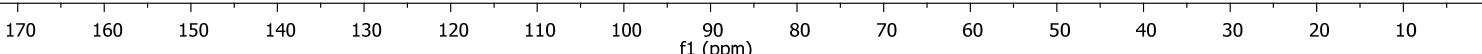

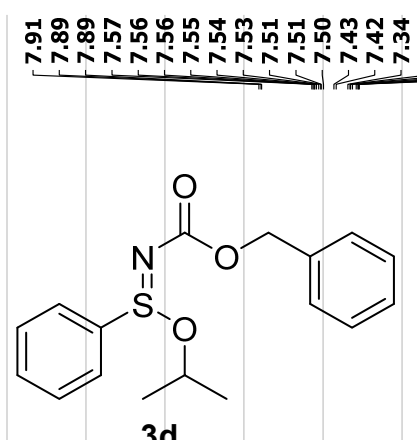

${ }^{1} \mathrm{H} \mathrm{NMR}, \mathrm{CDCl}_{3}, 500 \mathrm{MHz}$

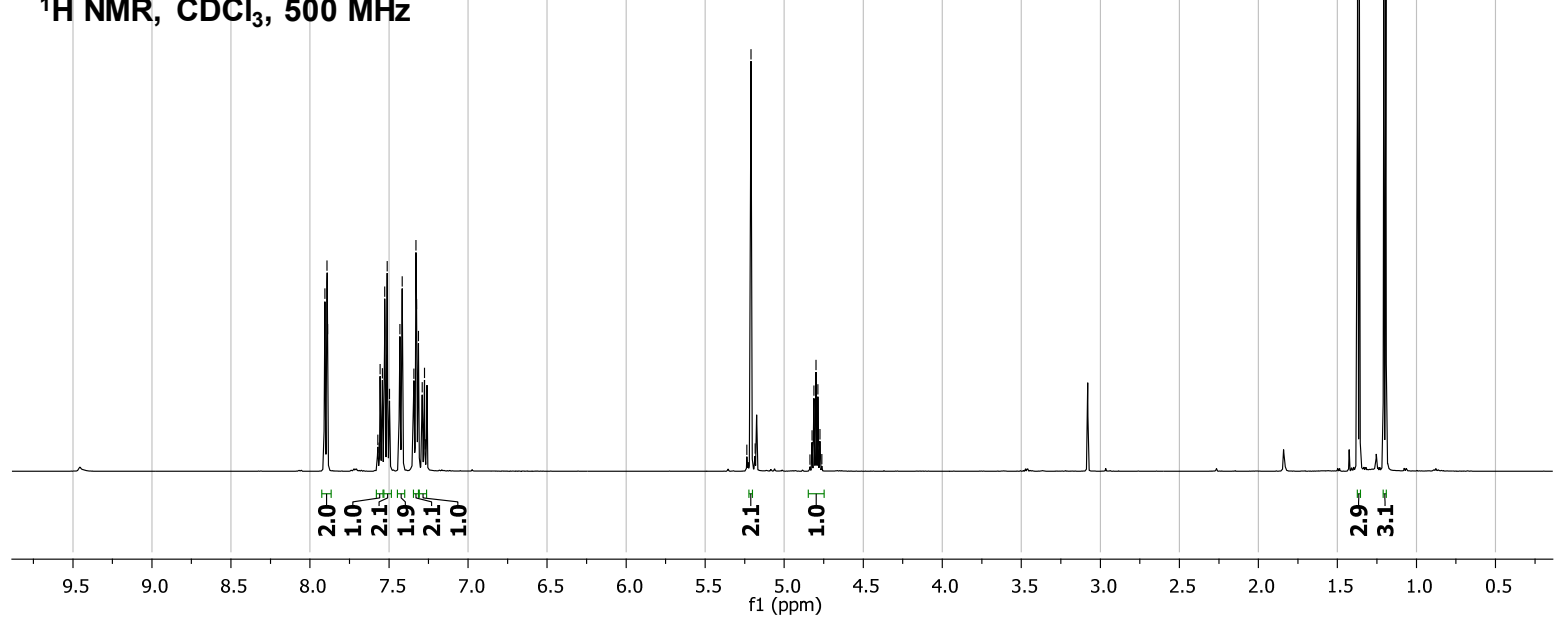



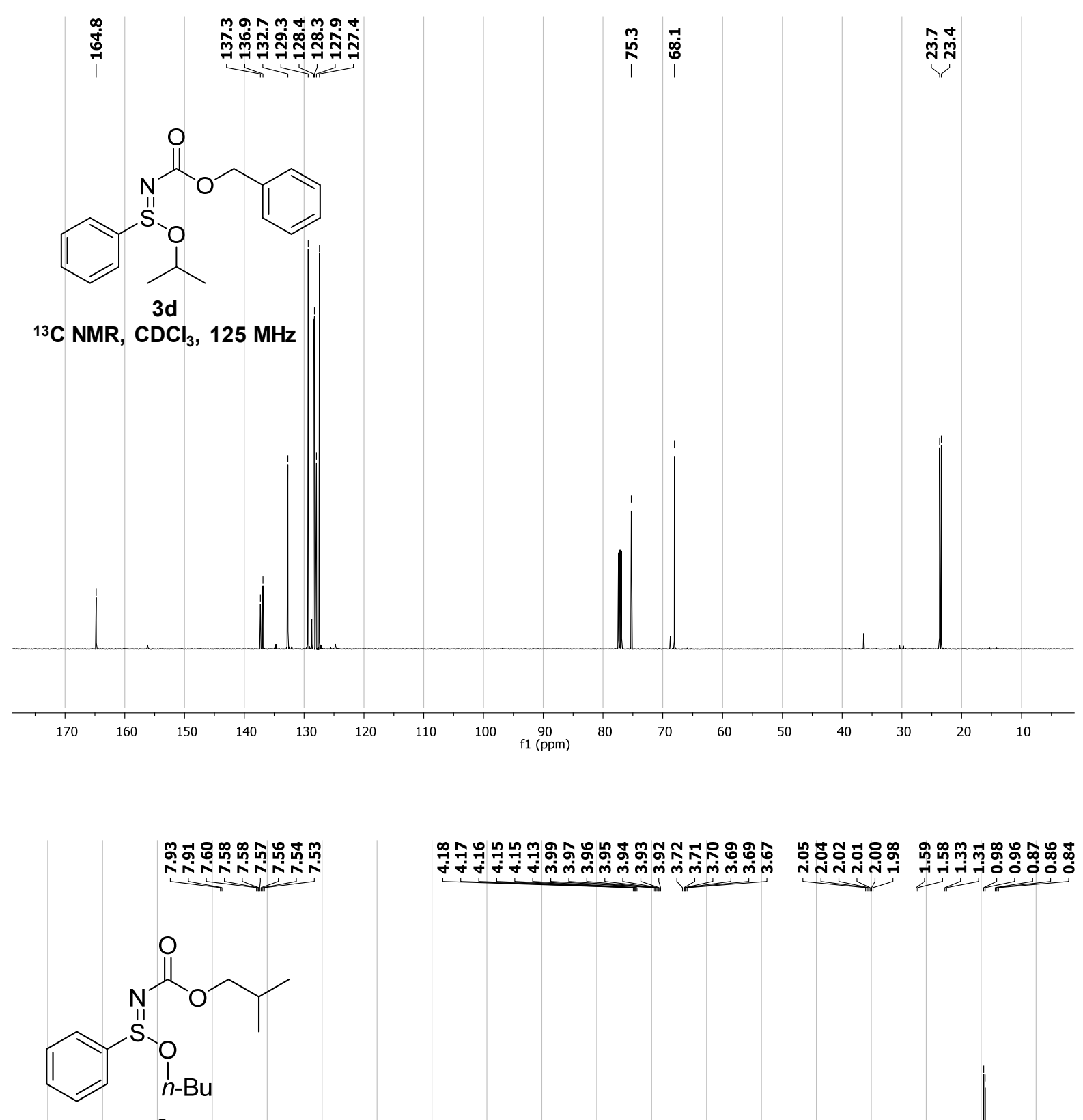

${ }^{1} \mathrm{H} \mathrm{NMR}, \mathrm{CDCl}_{3}, 500 \mathrm{MHz}$

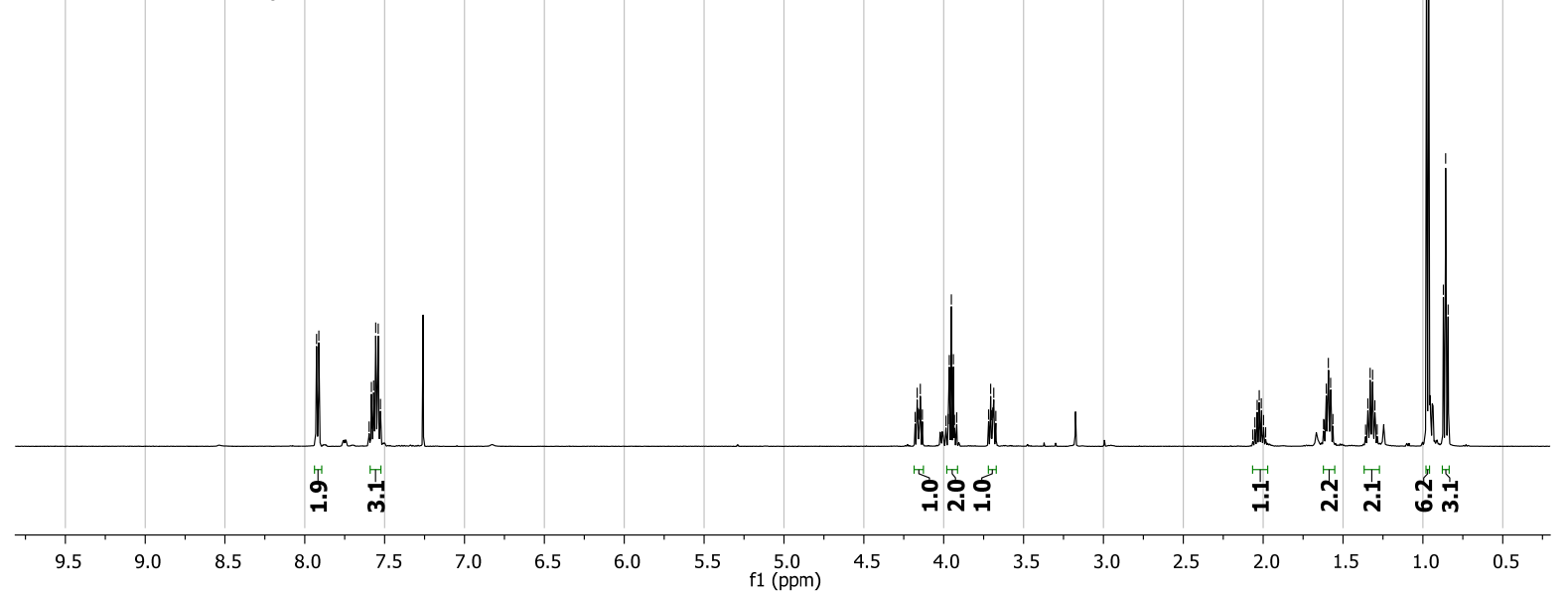




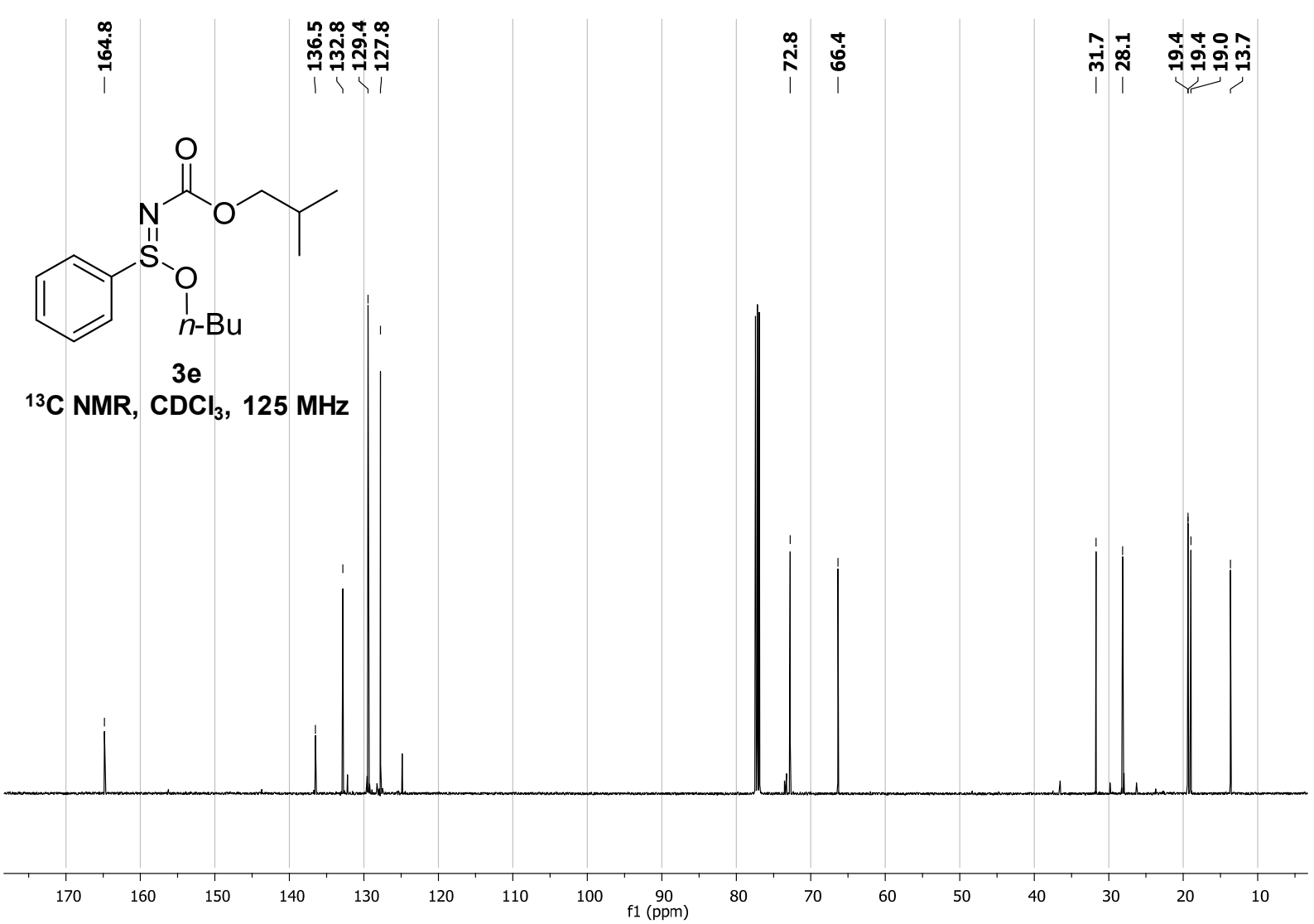

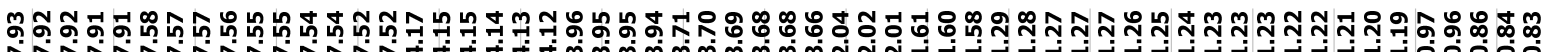<smiles>CC(C)COC(=O)/N=S(/OCC#CCCO)c1ccccc1</smiles>

${ }^{1} \mathrm{H} \mathrm{NMR}, \mathrm{CDCl}_{3}, 500 \mathrm{MHz}$

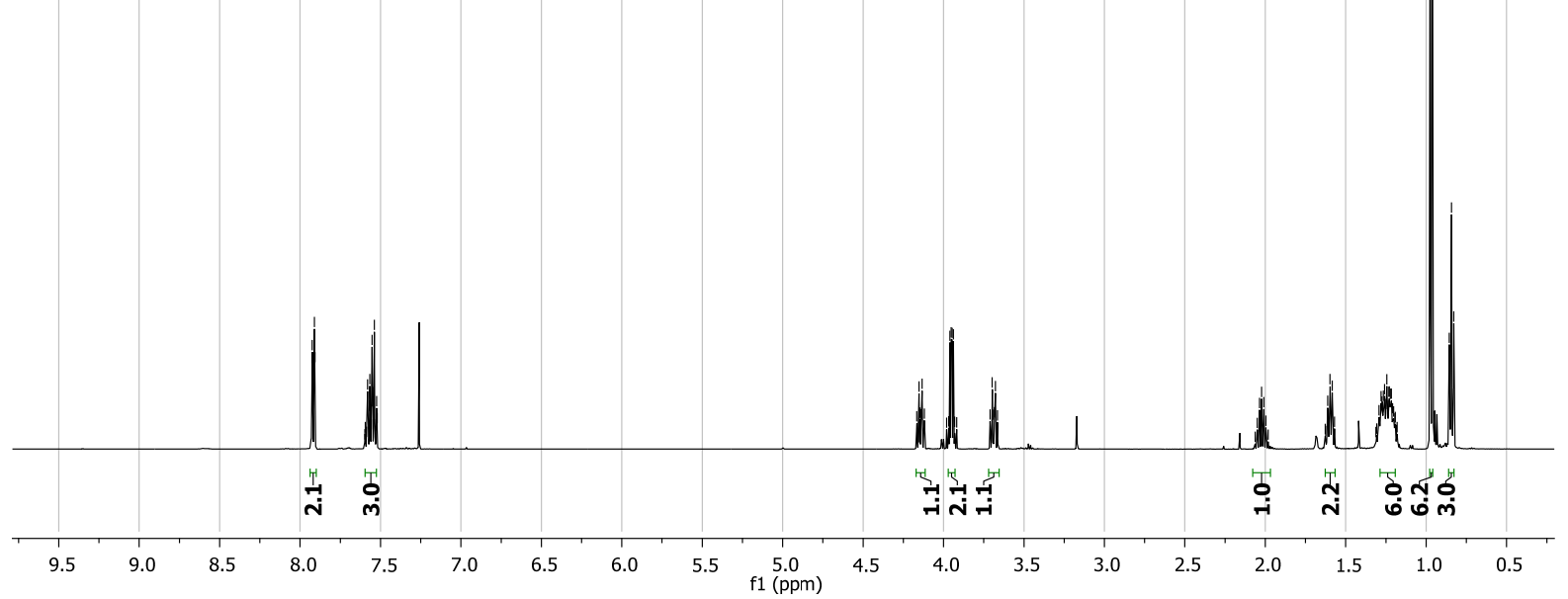



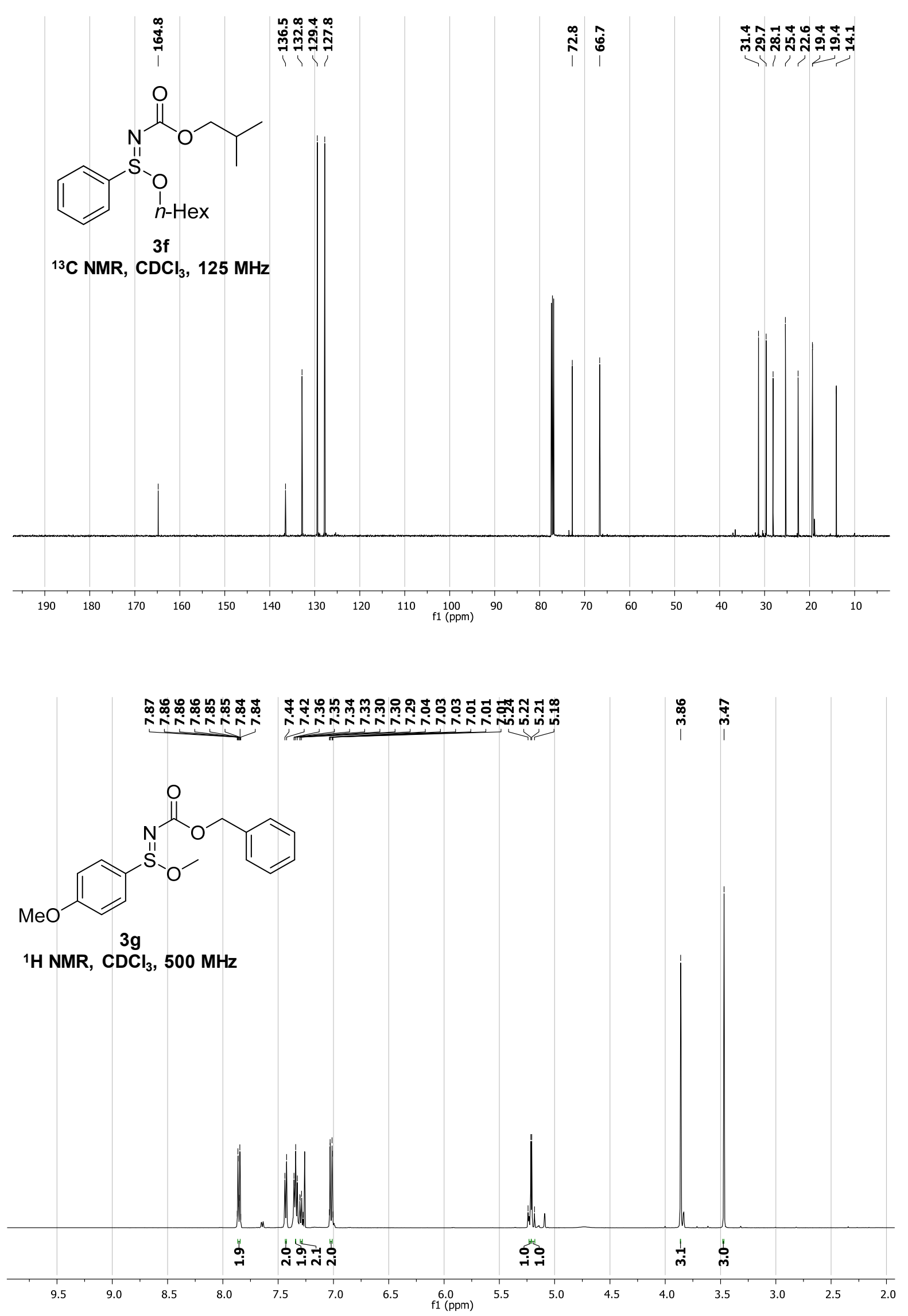

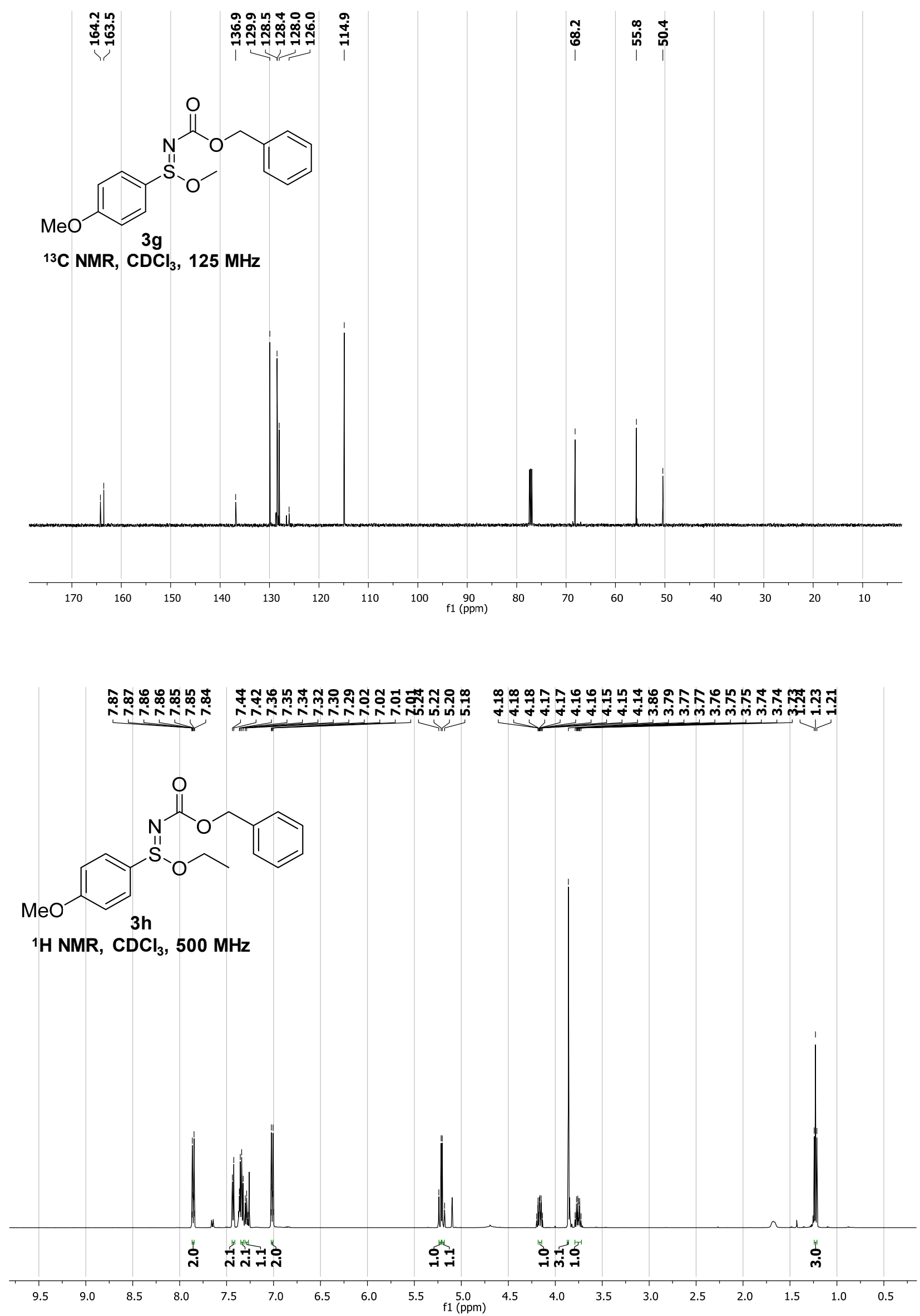

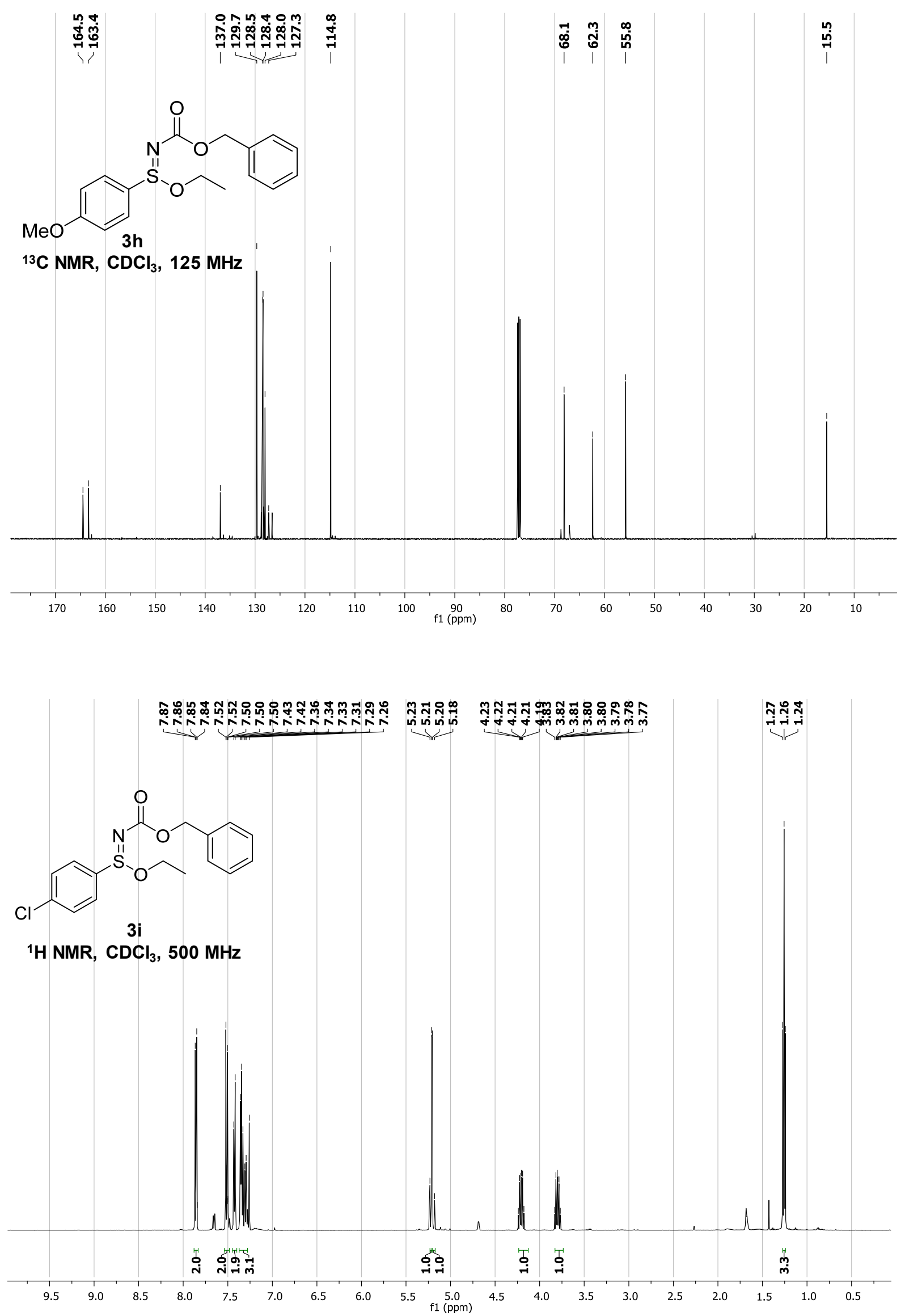

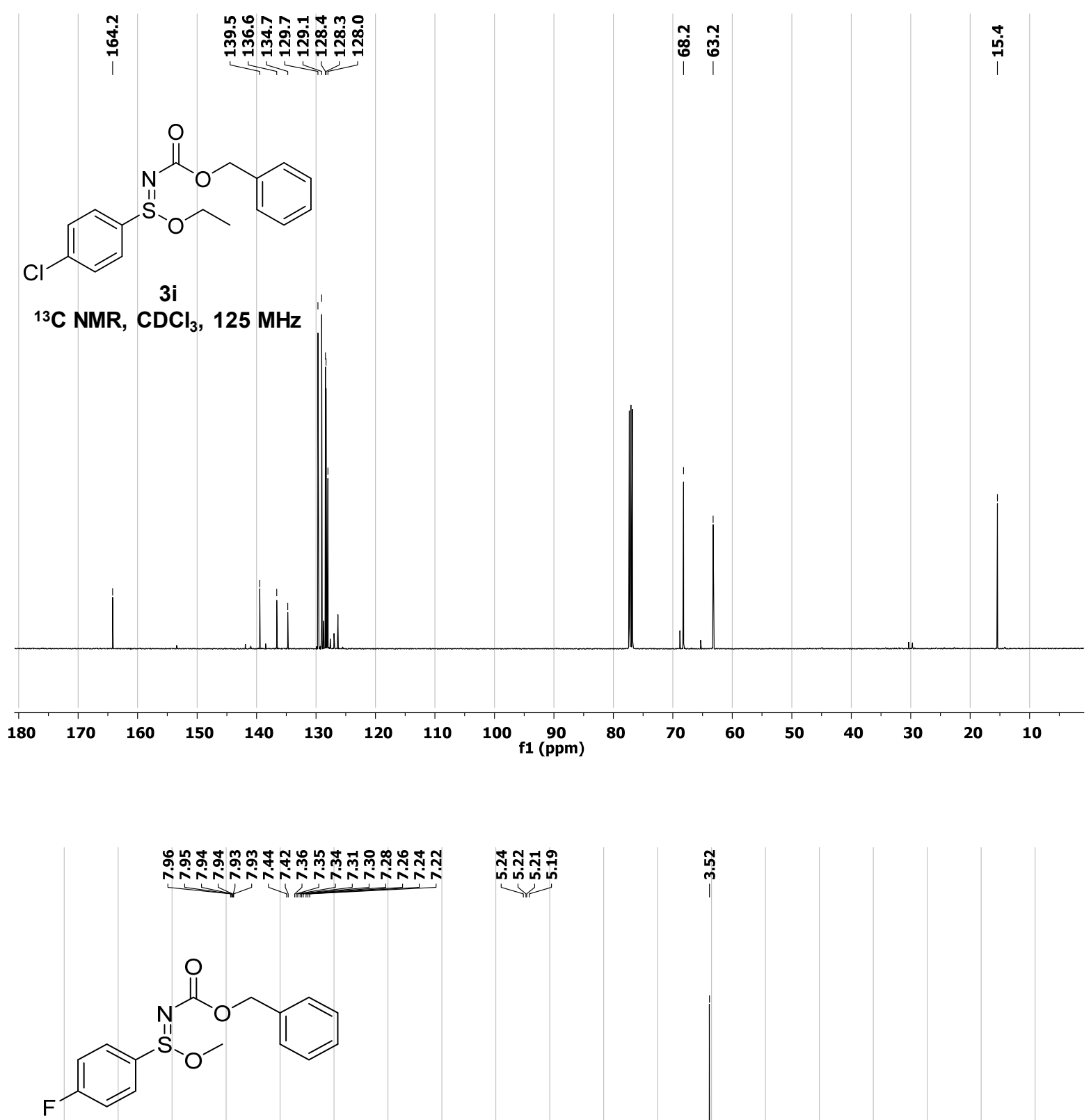

${ }^{1} \mathrm{H} \mathrm{NMR}, \mathrm{CDCl}_{3}, 500 \mathrm{MHz}$

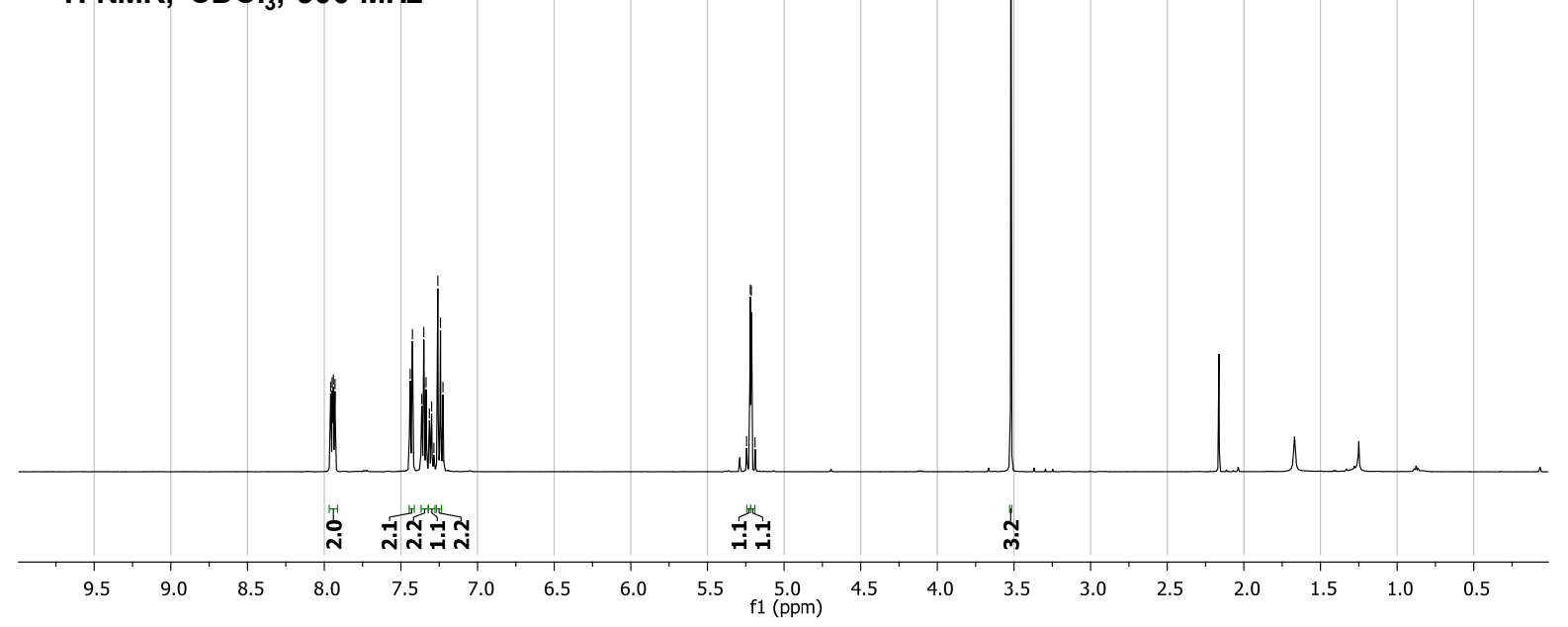



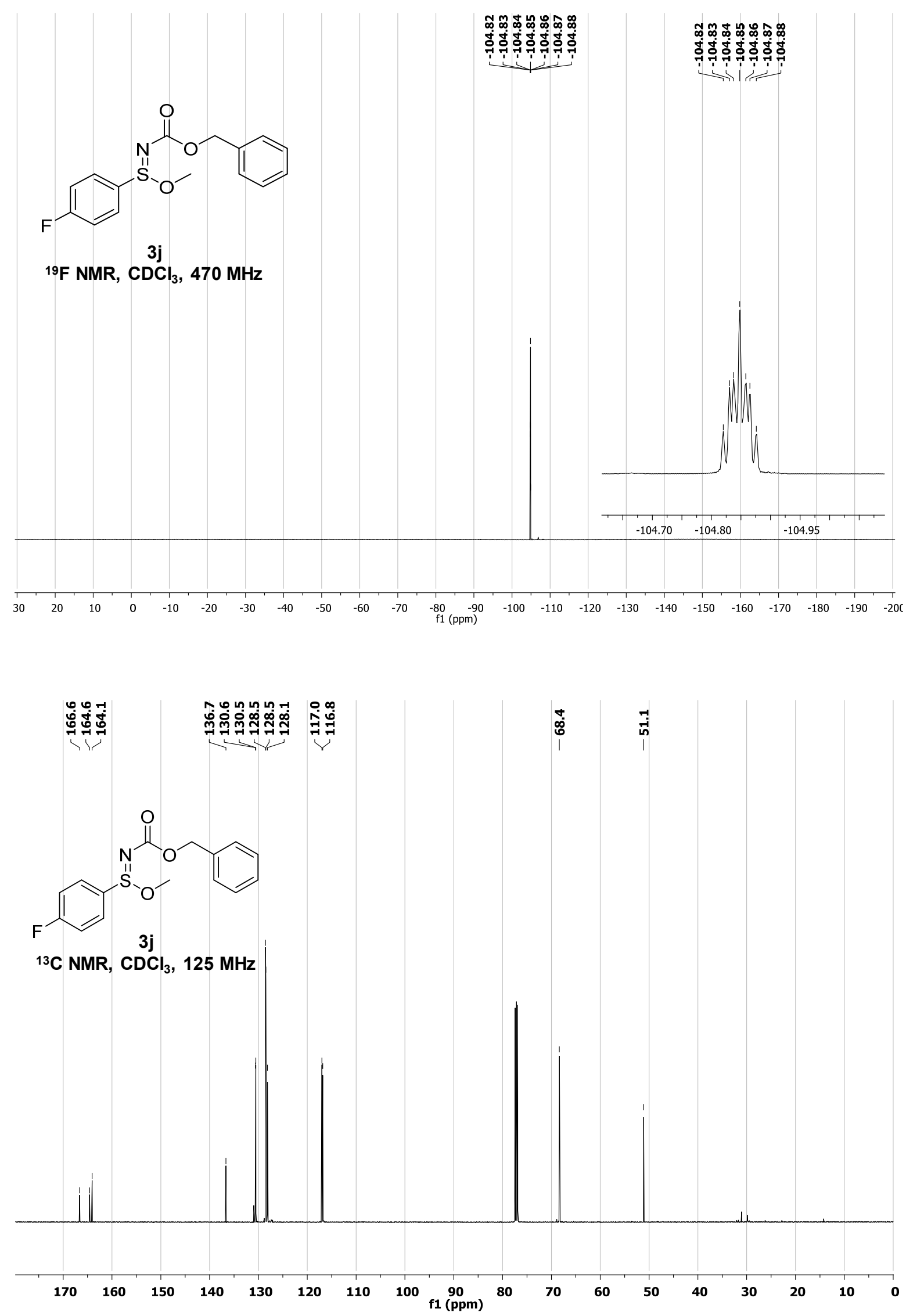

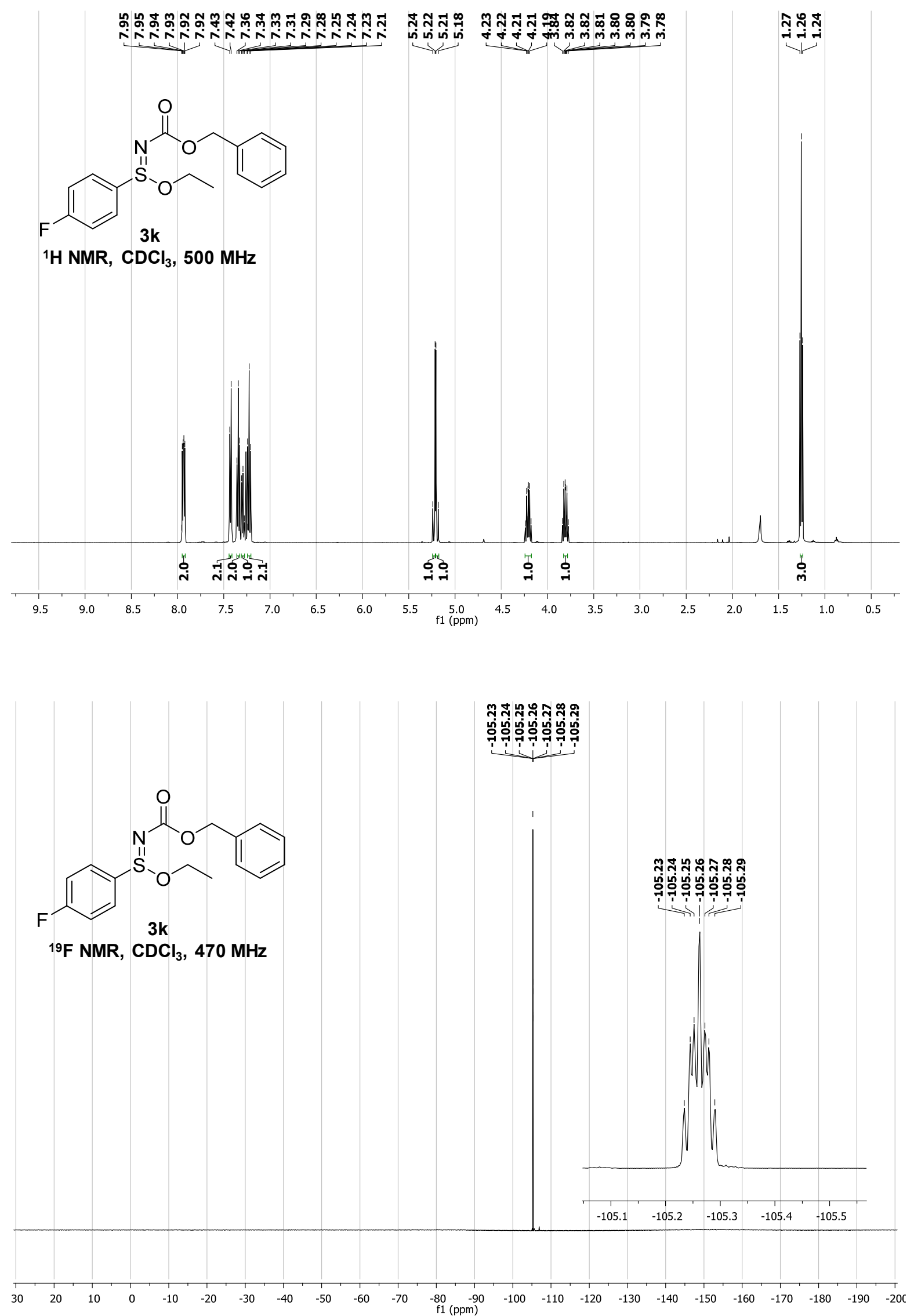

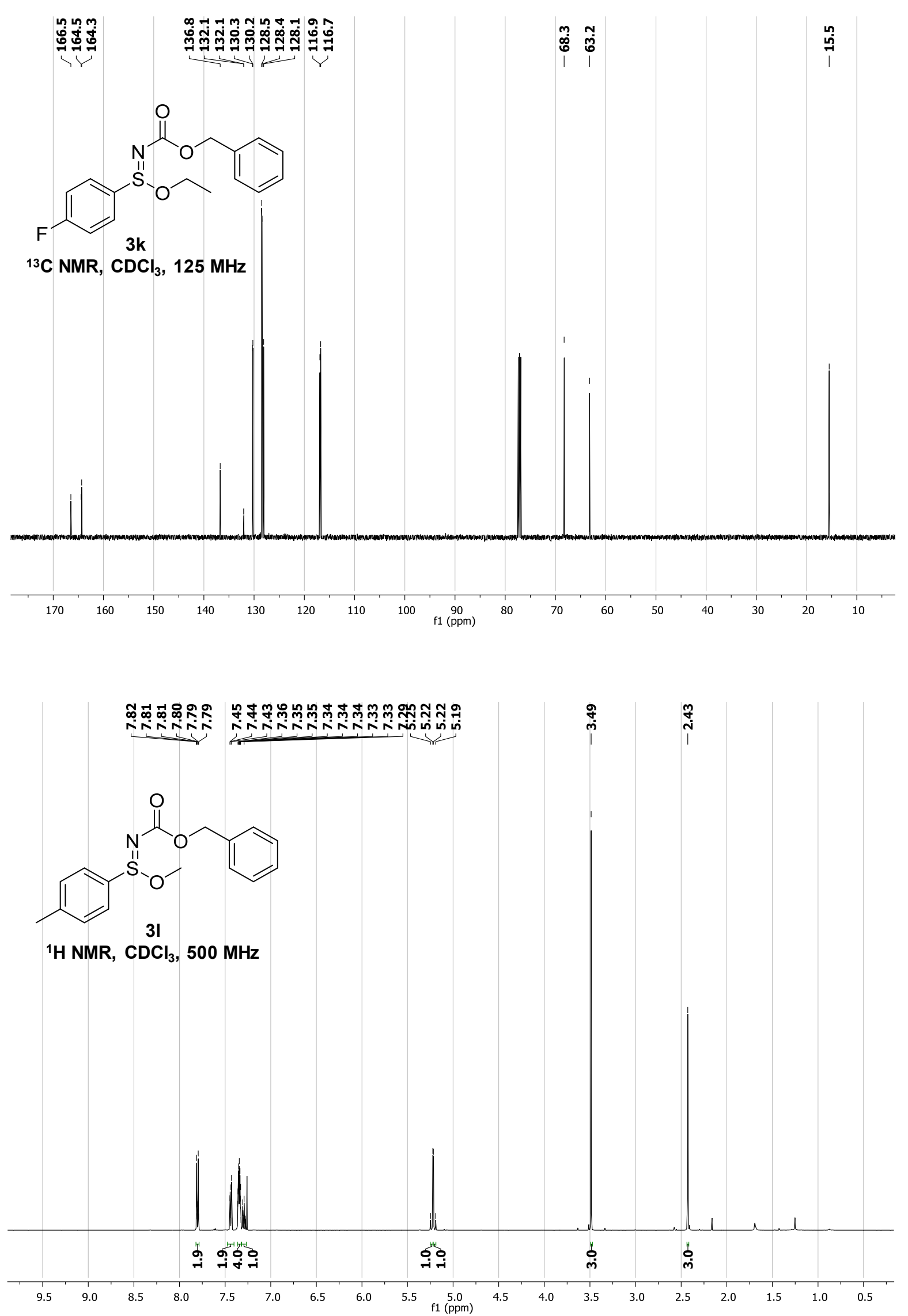

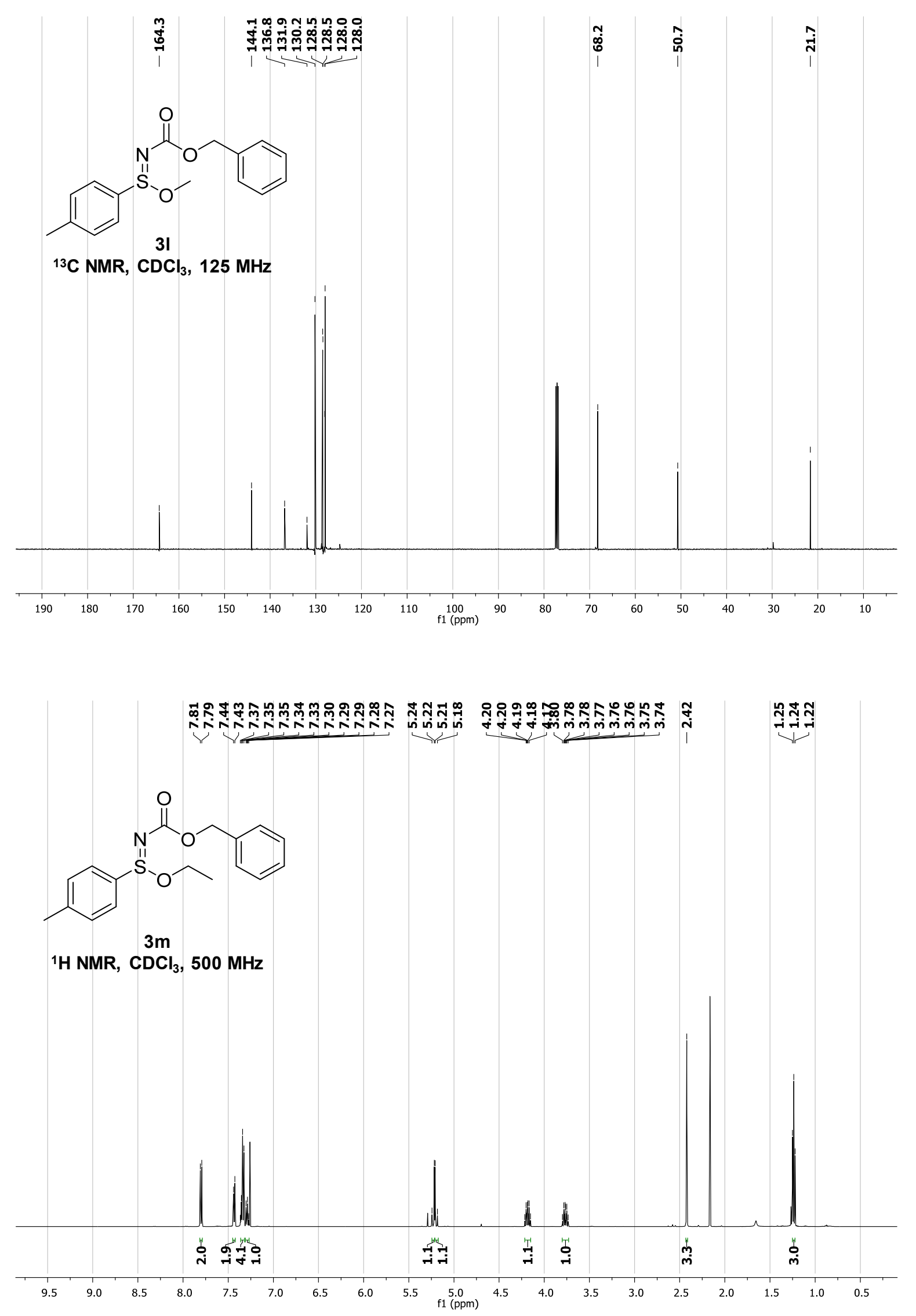

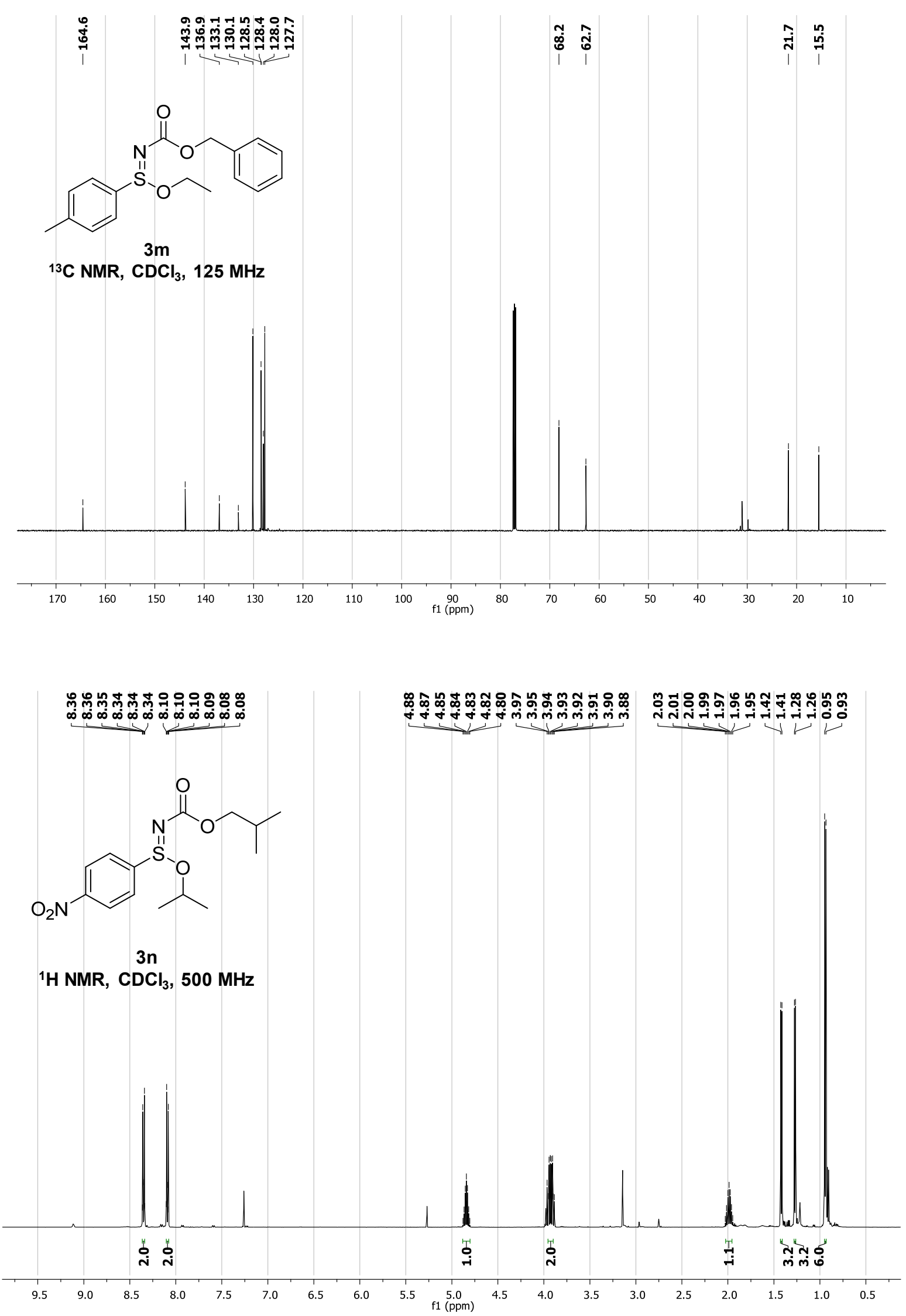

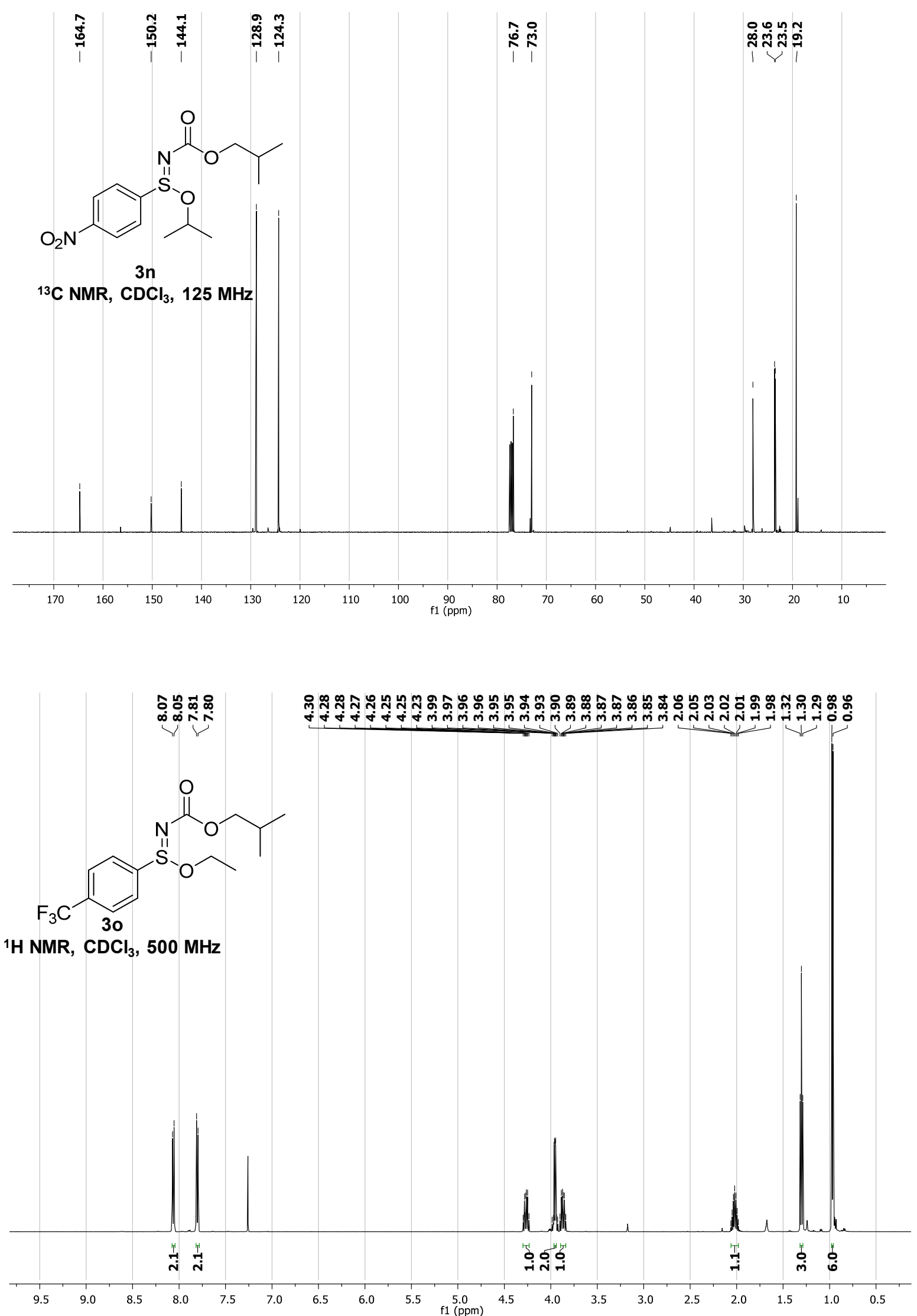

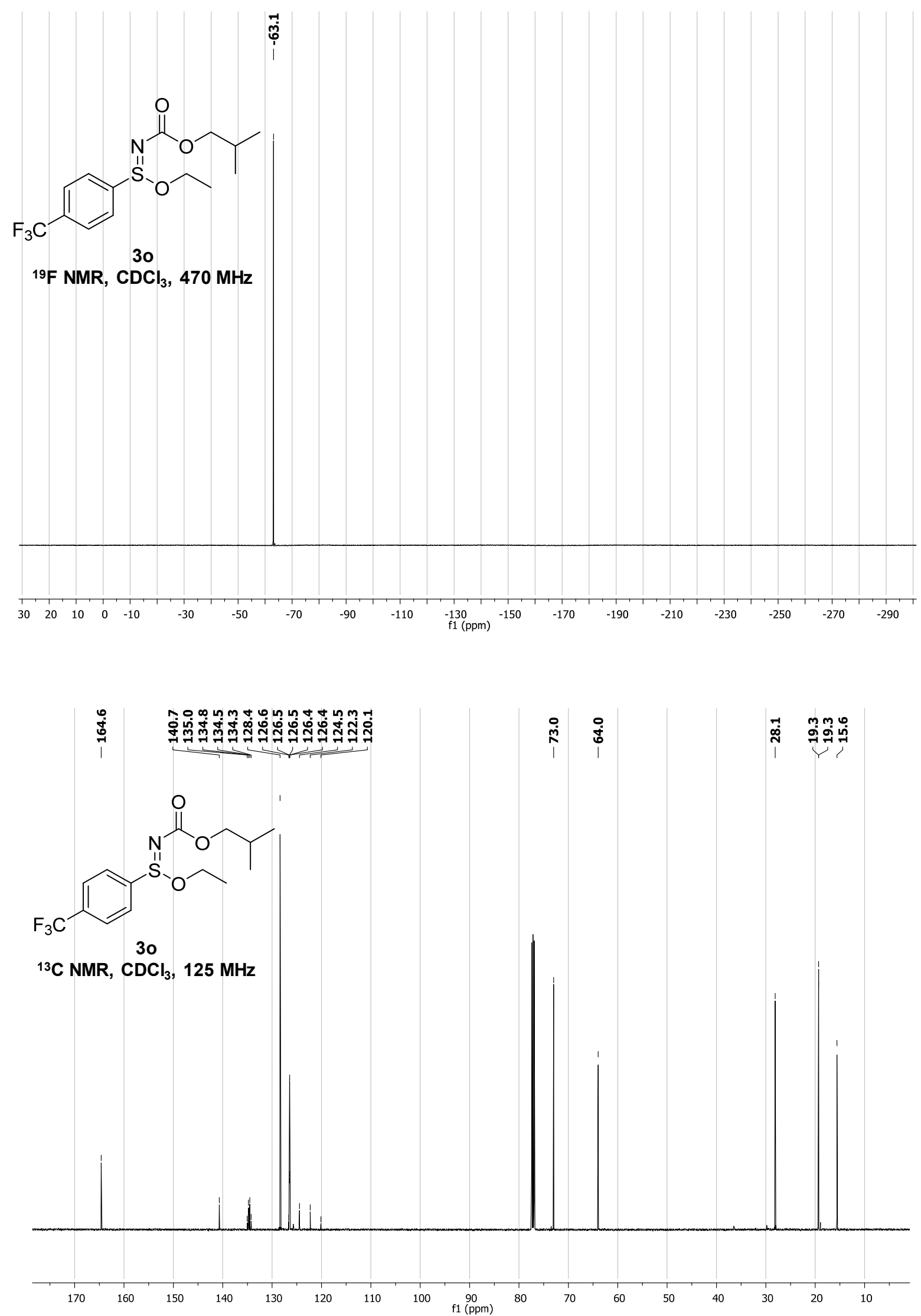

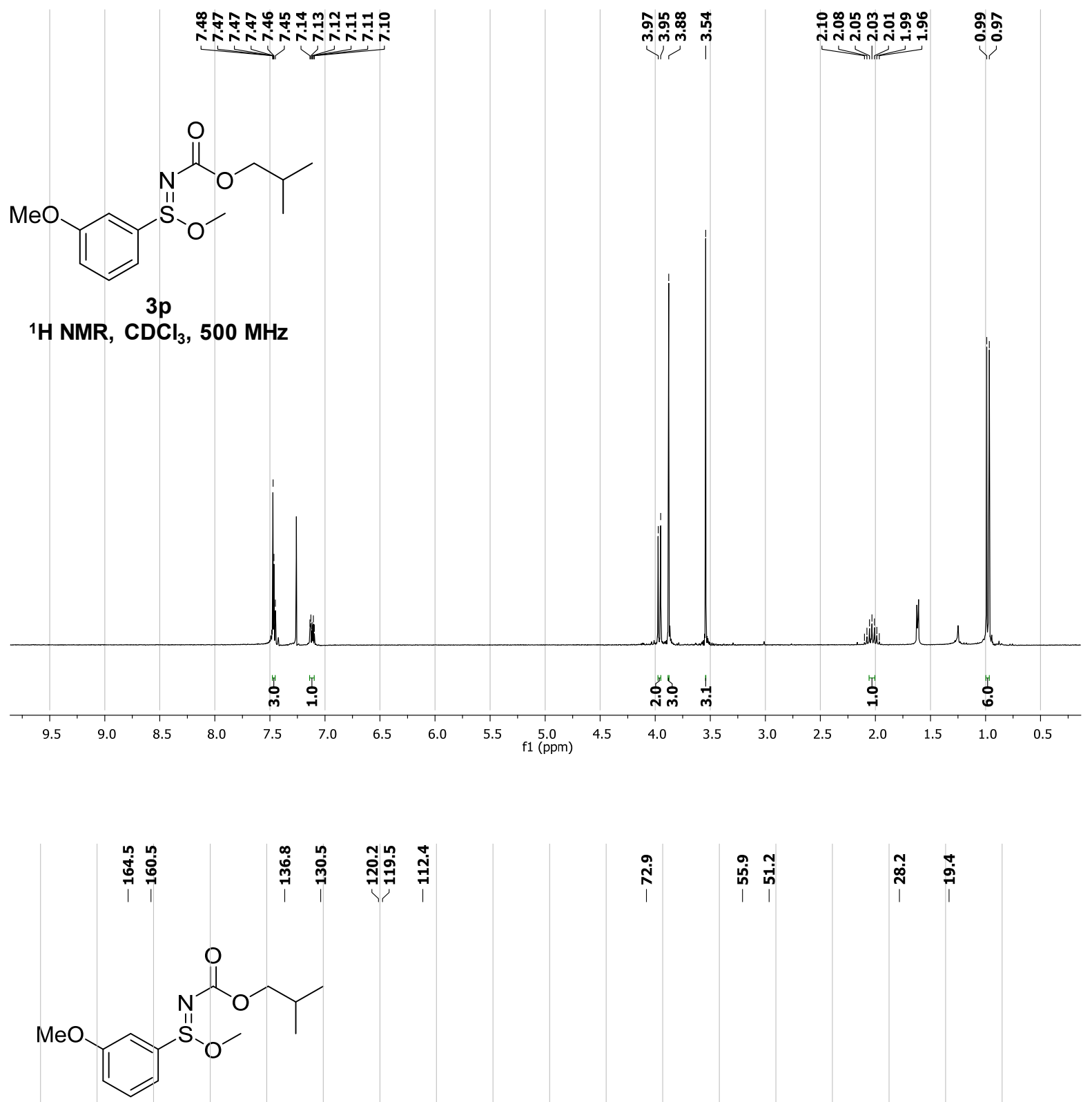

${ }^{13} \mathrm{C}$ NMR, $\stackrel{3 p}{\mathrm{CDC}_{3}}, 125 \mathrm{MHz}$

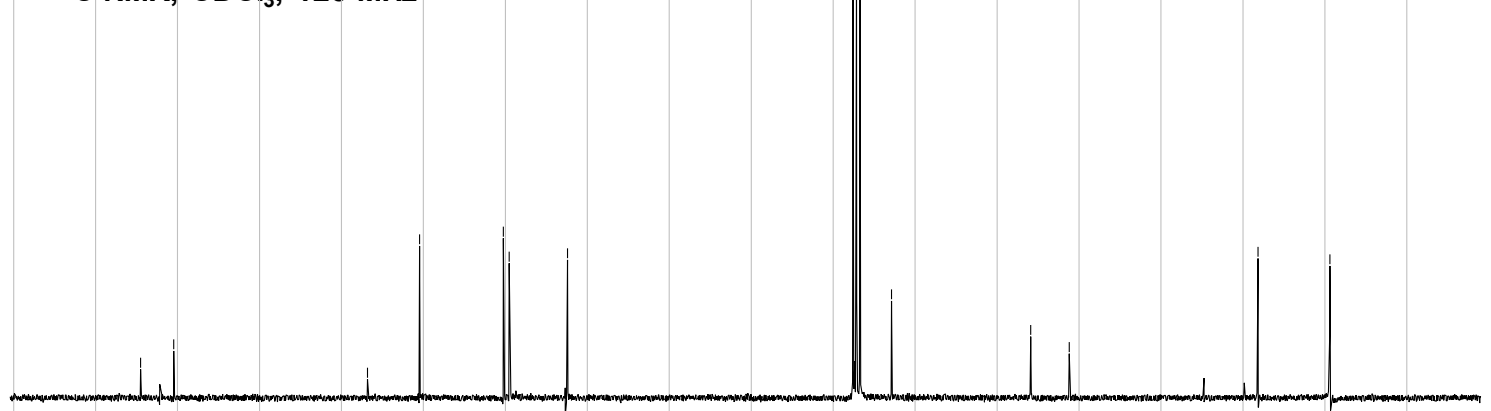



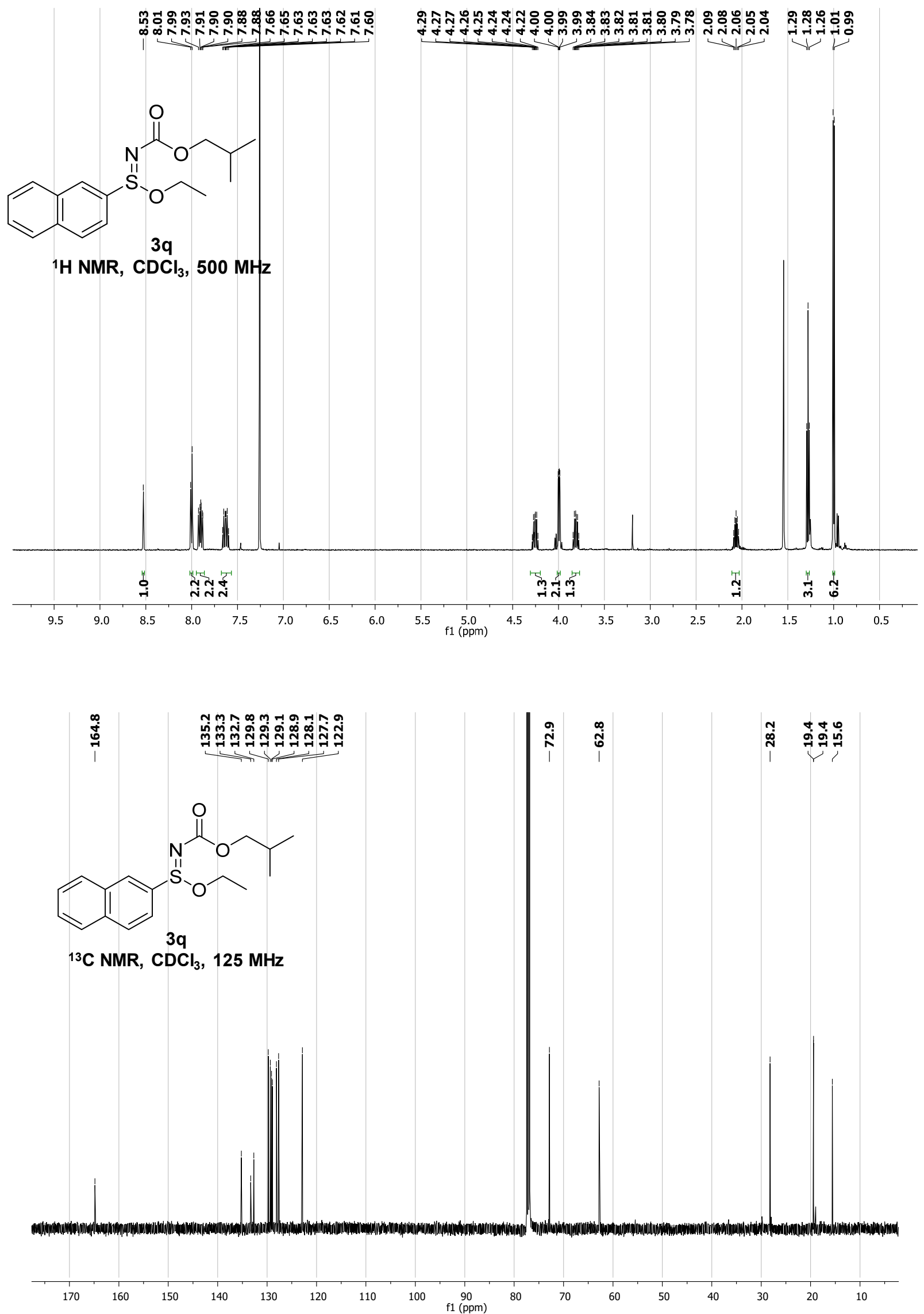
<smiles>CC(C)COC(=O)/N=S(\OC(C)C)C1CCCCC1</smiles>

$3 r$

${ }^{1} \mathrm{H} \mathrm{NMR}, \mathrm{CDCl}_{3}, 500 \mathrm{MHz}$
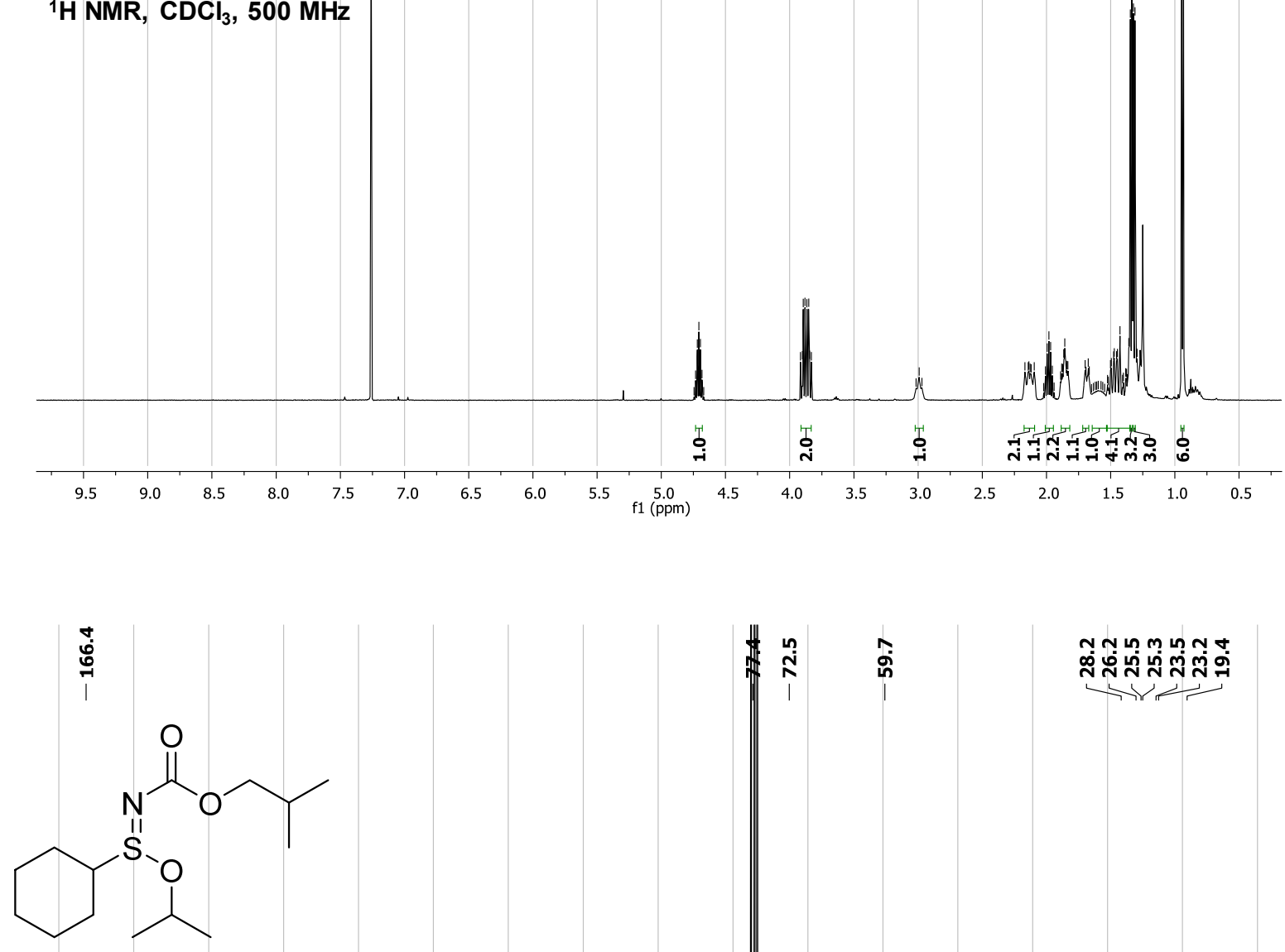

$3 r$

${ }^{13} \mathrm{C} \mathrm{NMR}, \mathrm{CDCl}_{3}, 125 \mathrm{MHz}$

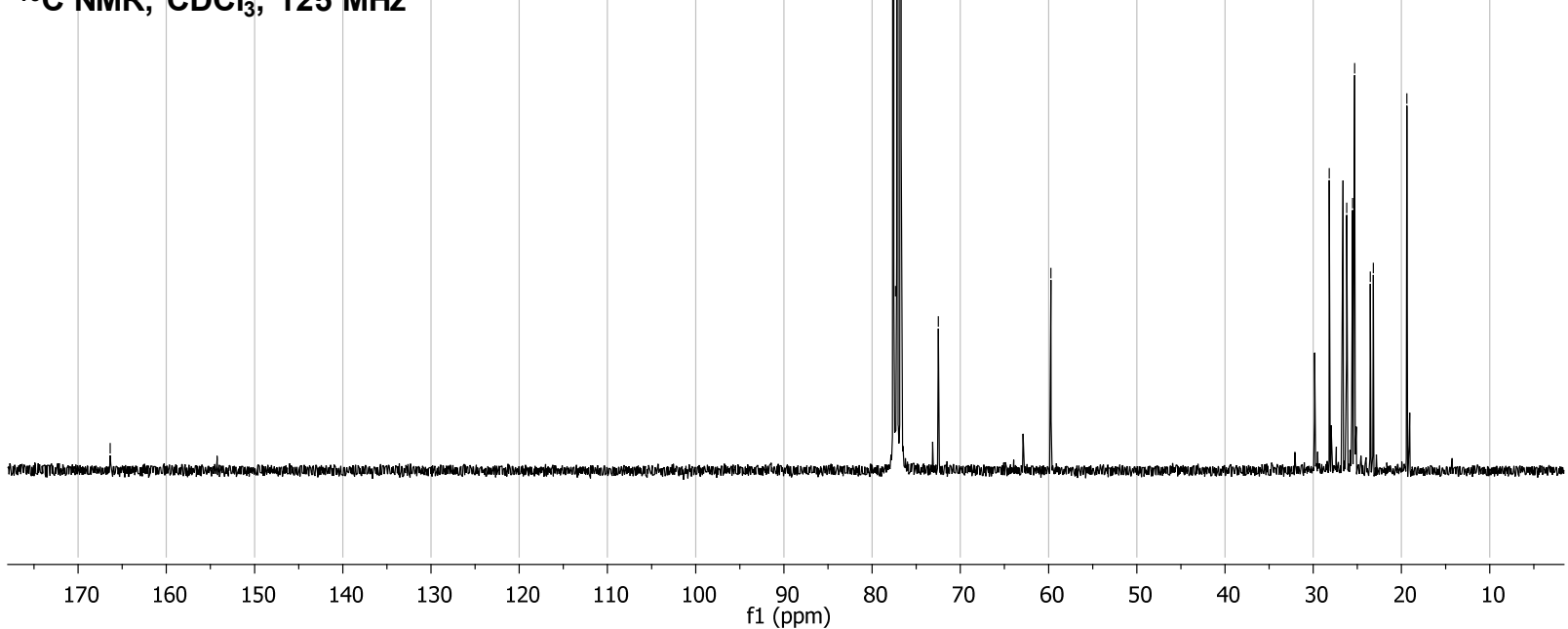


<smiles>CCCCO/S(=N\C(=O)OCC(C)C)C1CCCCC1</smiles>

${ }^{1} \mathrm{H} \mathrm{NMR}, \mathrm{CDCl}_{3}, 500 \mathrm{MHz}$

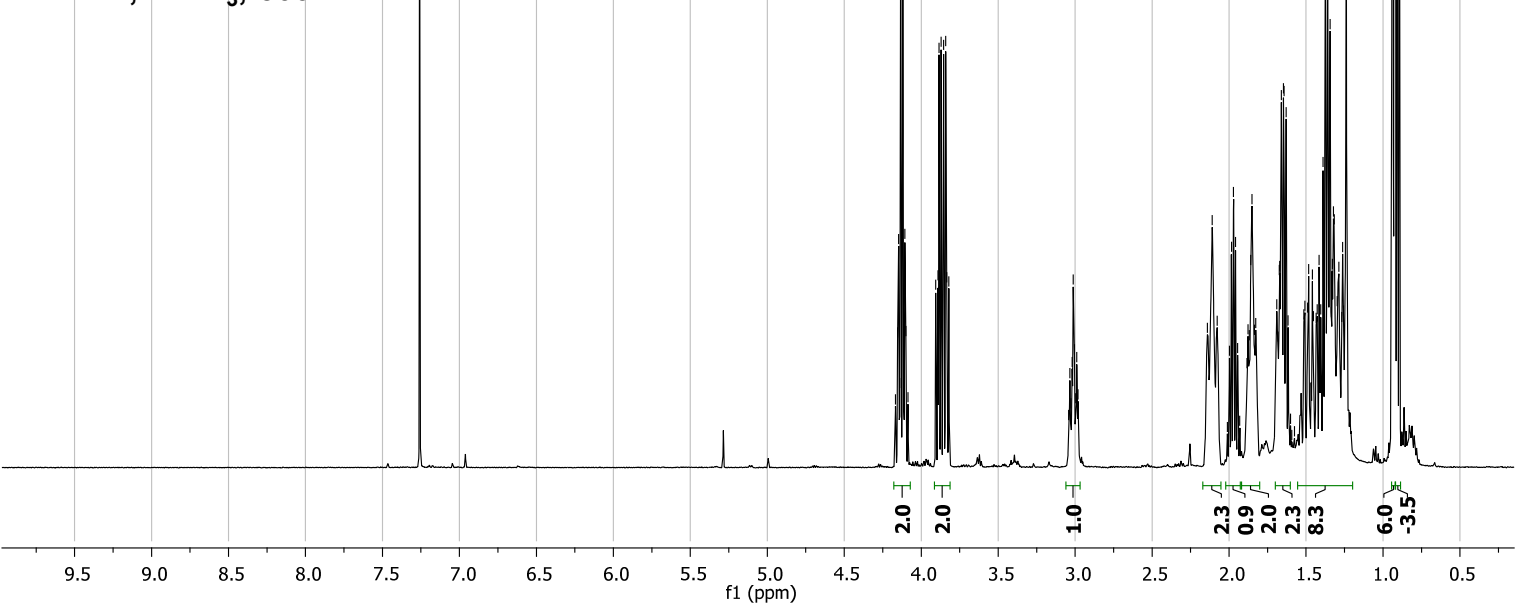<smiles>CCCCO/S(=N\C(=O)OCC(C)C)C1CCCCC1</smiles>

${ }^{13} \mathrm{C}$ NMR, $\mathrm{CDCl}_{3}, 125 \mathrm{MHz}$

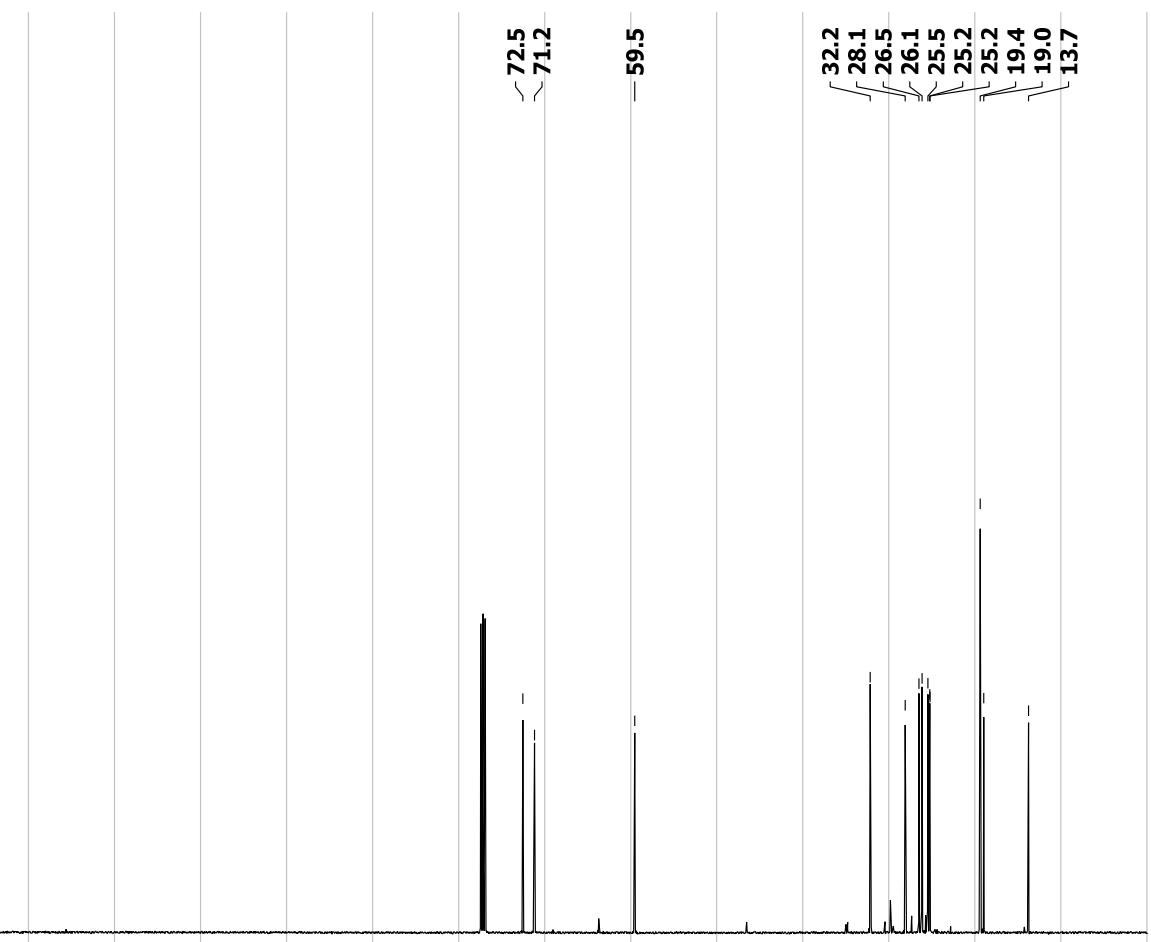

$\begin{array}{rllllllllllllllllll}180 & 170 & 160 & 150 & 140 & 130 & 120 & 110 & 100 & \begin{array}{c}90 \\ \mathrm{f} 1(\mathrm{ppm})\end{array} & 80 & 70 & 60 & 50 & 40 & 30 & 20 & 10 & 0\end{array}$ 

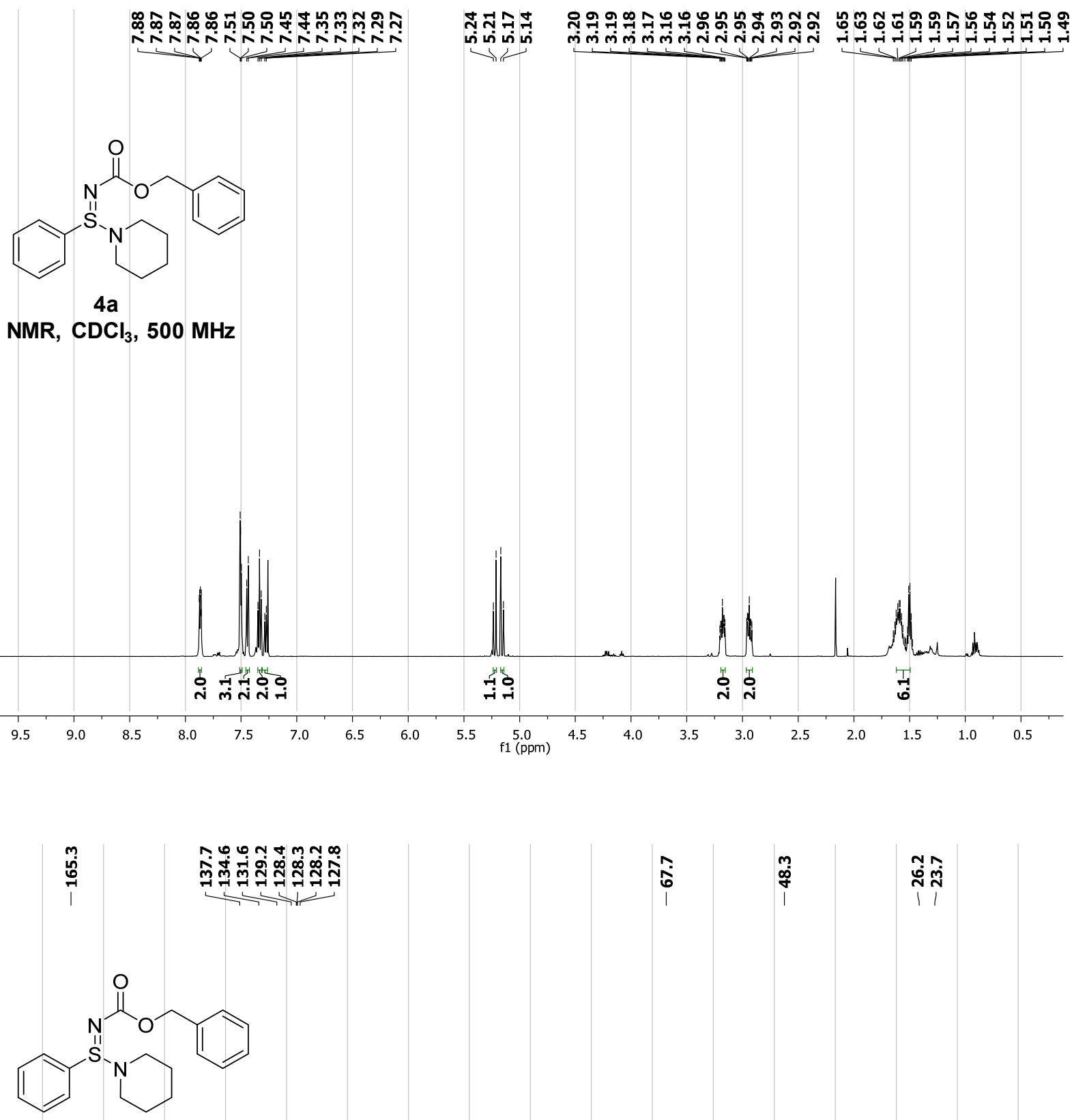

$4 a$

${ }^{13} \mathrm{C}$ NMR, $\mathrm{CDCl}_{3}, 125 \mathrm{MHz}$

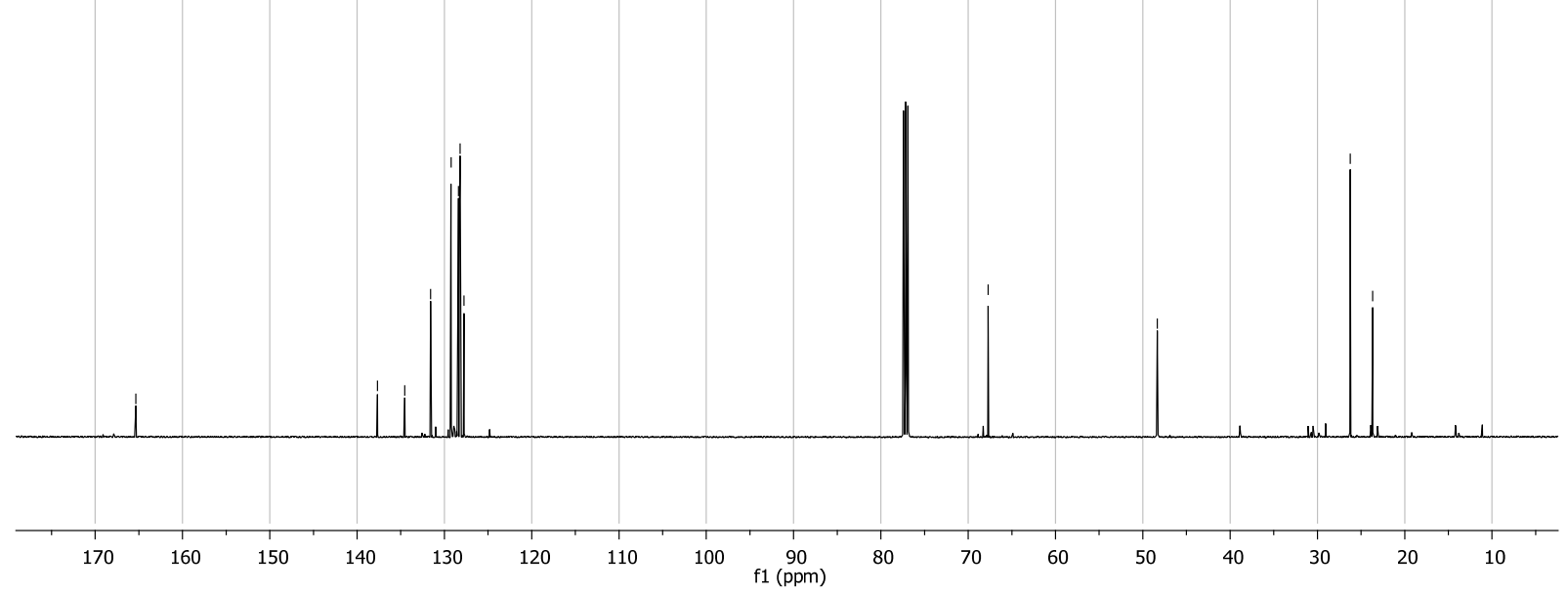



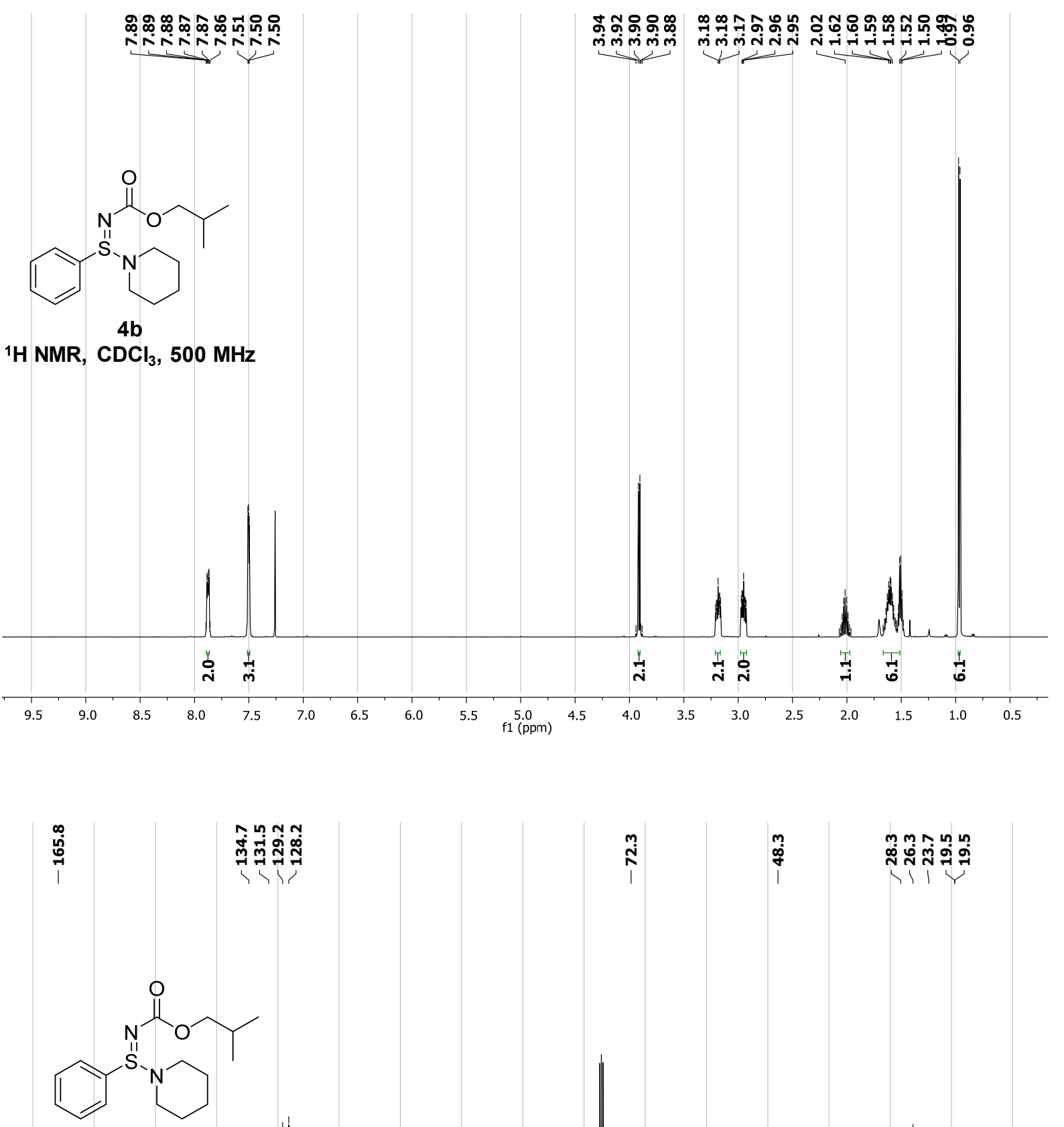

4b

${ }^{13} \mathrm{C}$ NMR, $\mathrm{CDCl}_{3}, 125 \mathrm{MHz}$

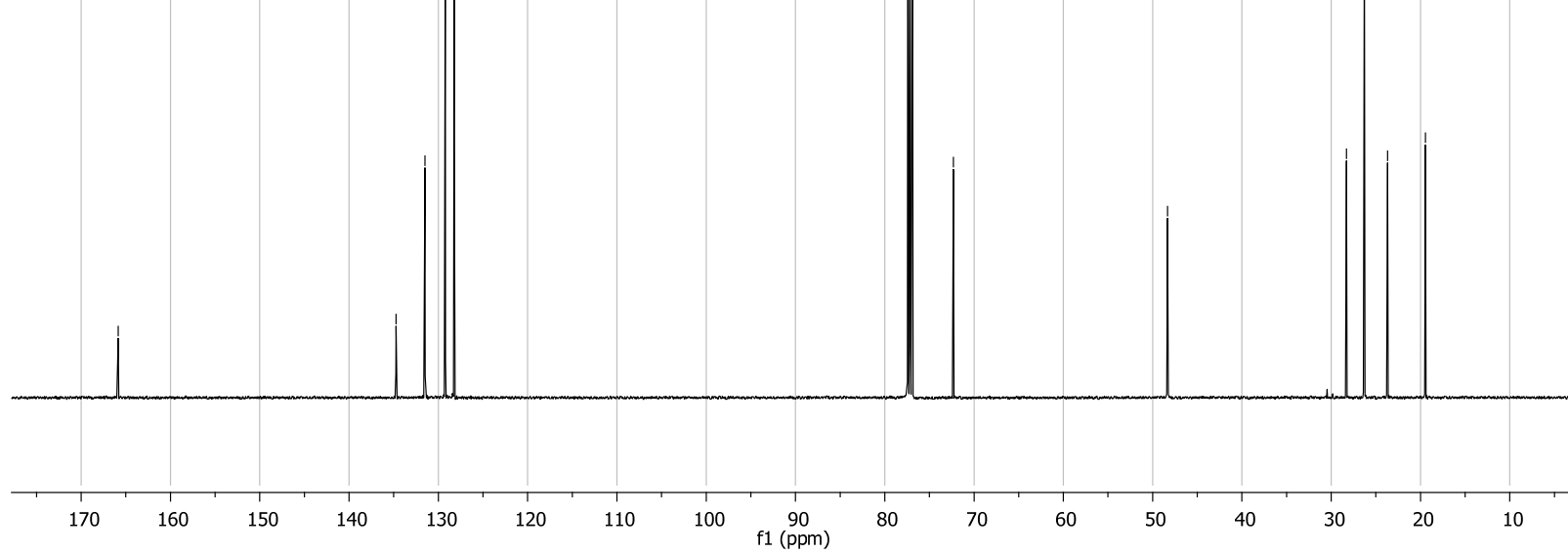


<smiles>COc1ccc(S(=NC(=O)OCc2ccccc2)N2CCCCC2)cc1</smiles>

${ }^{1} \mathrm{H} \mathrm{NMR}, \mathrm{CDCl}_{3}, 500 \mathrm{MHz}$
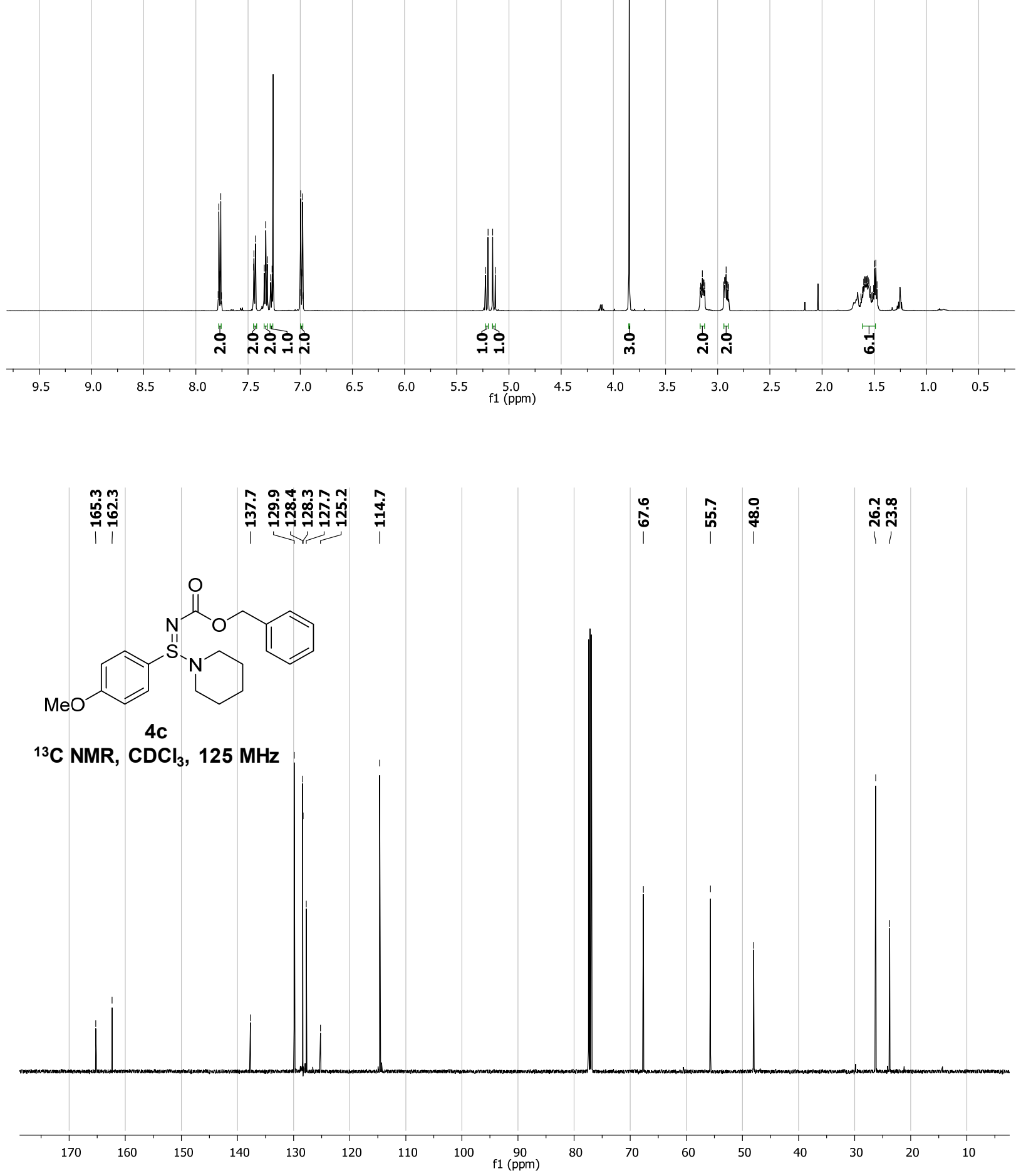


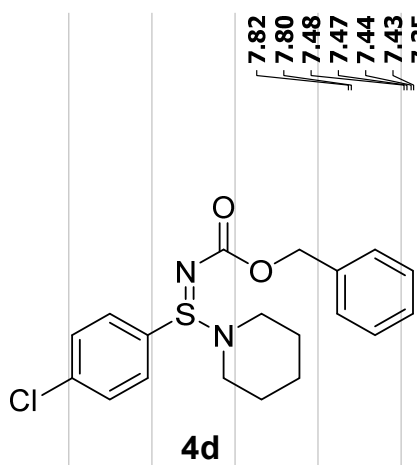

${ }^{1} \mathrm{H} \mathrm{NMR}, \mathrm{CDCl}_{3}, 500 \mathrm{MHz}$
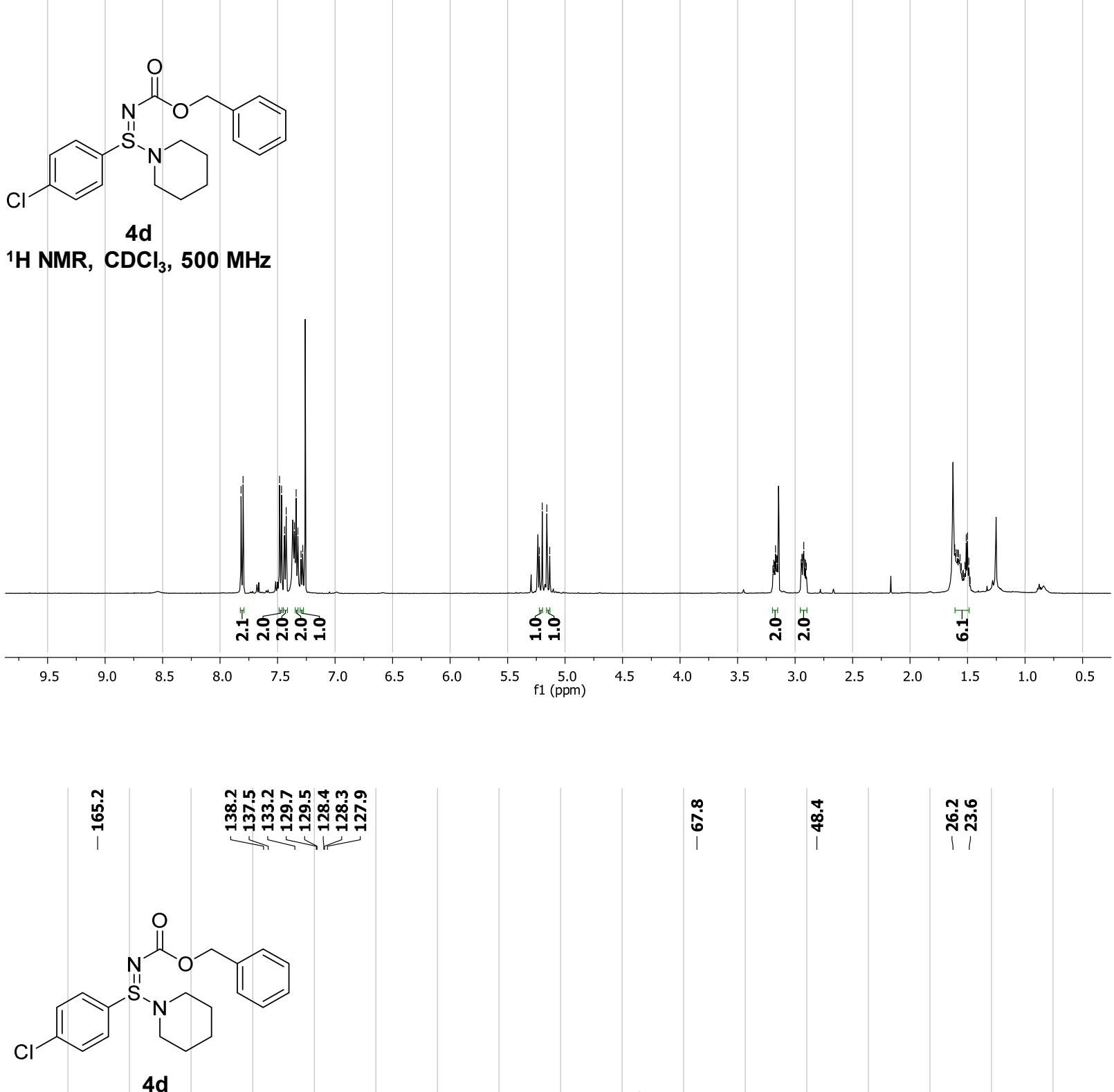

${ }^{13} \mathrm{C} \mathrm{NMR,} \mathrm{CDCl}_{3}, 125 \mathrm{MHz}$
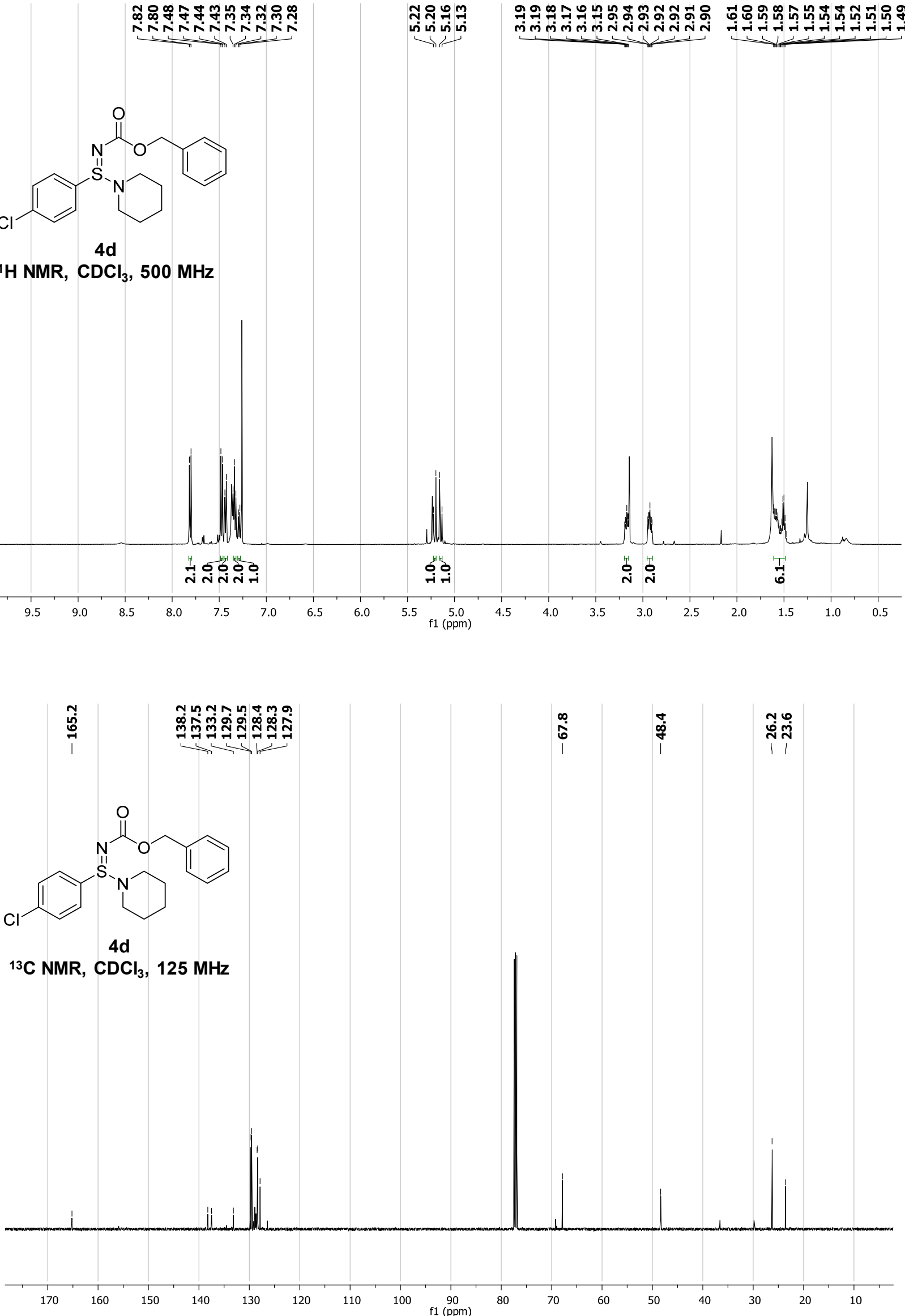

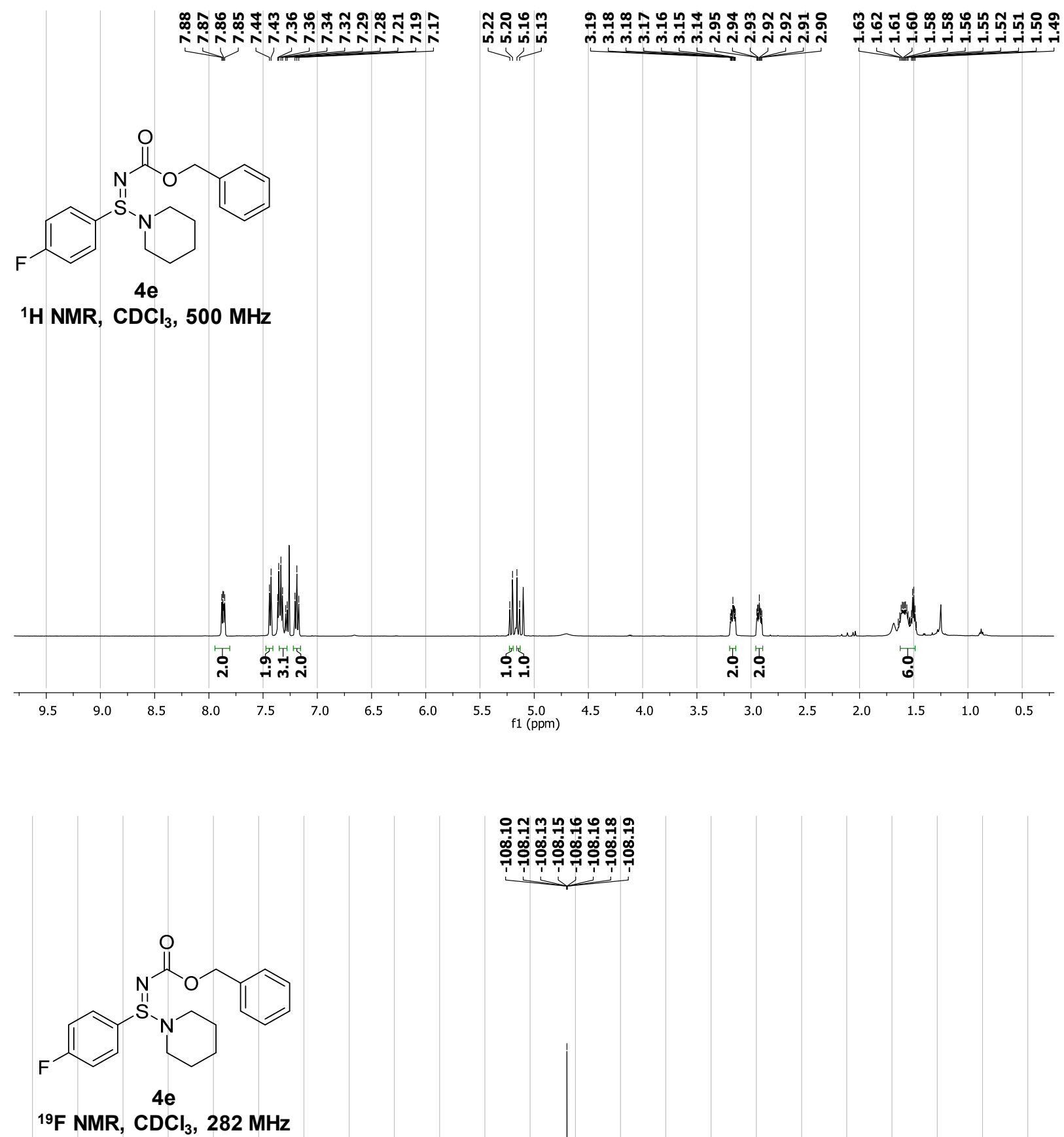

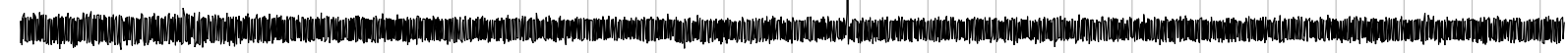

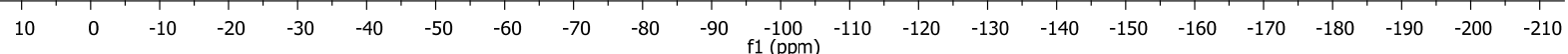



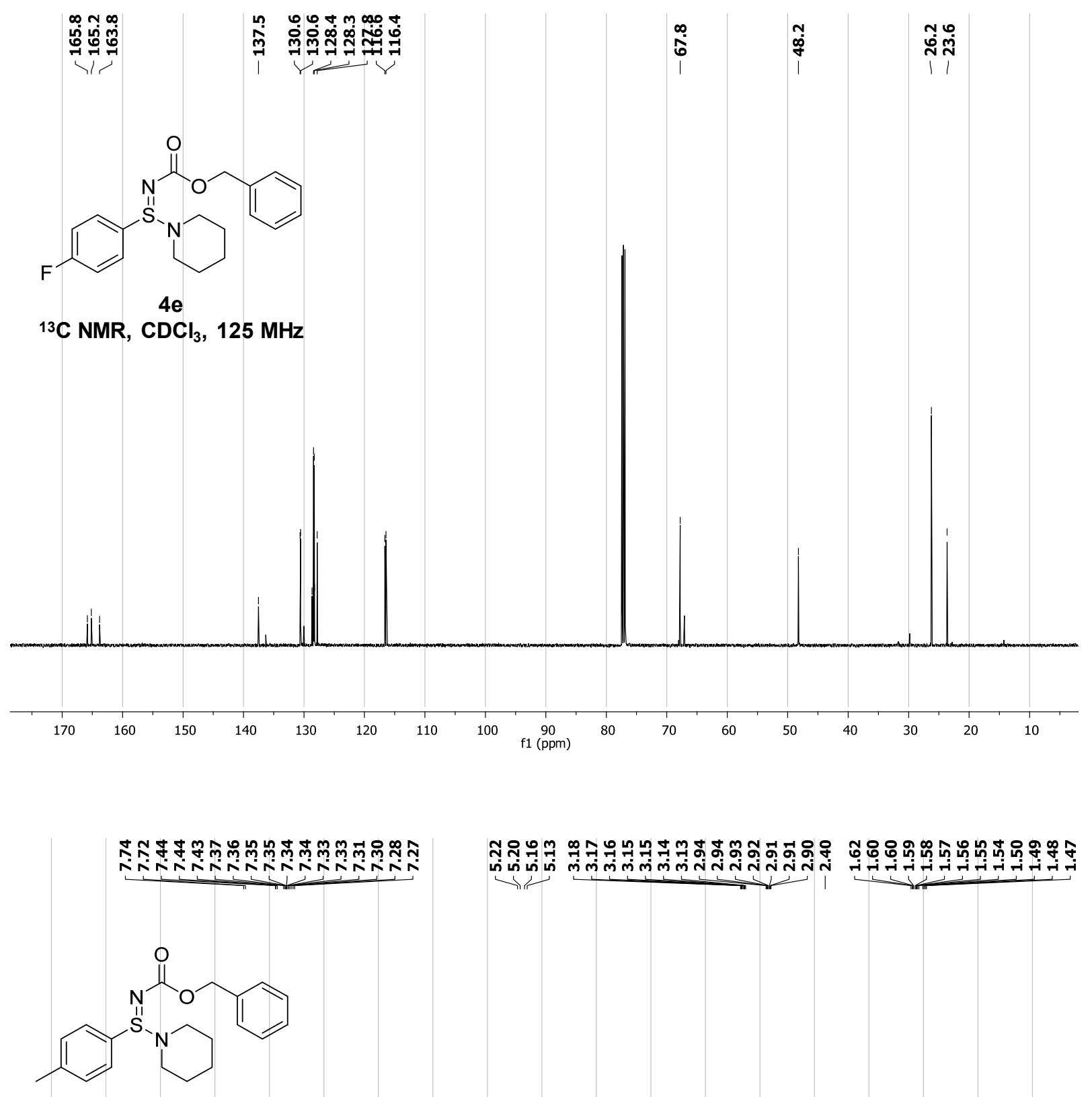

$\stackrel{4 f}{1}{ }^{1} \mathrm{H}$ NMR, $\mathrm{CDCl}_{3}, 500 \mathrm{MHz}$

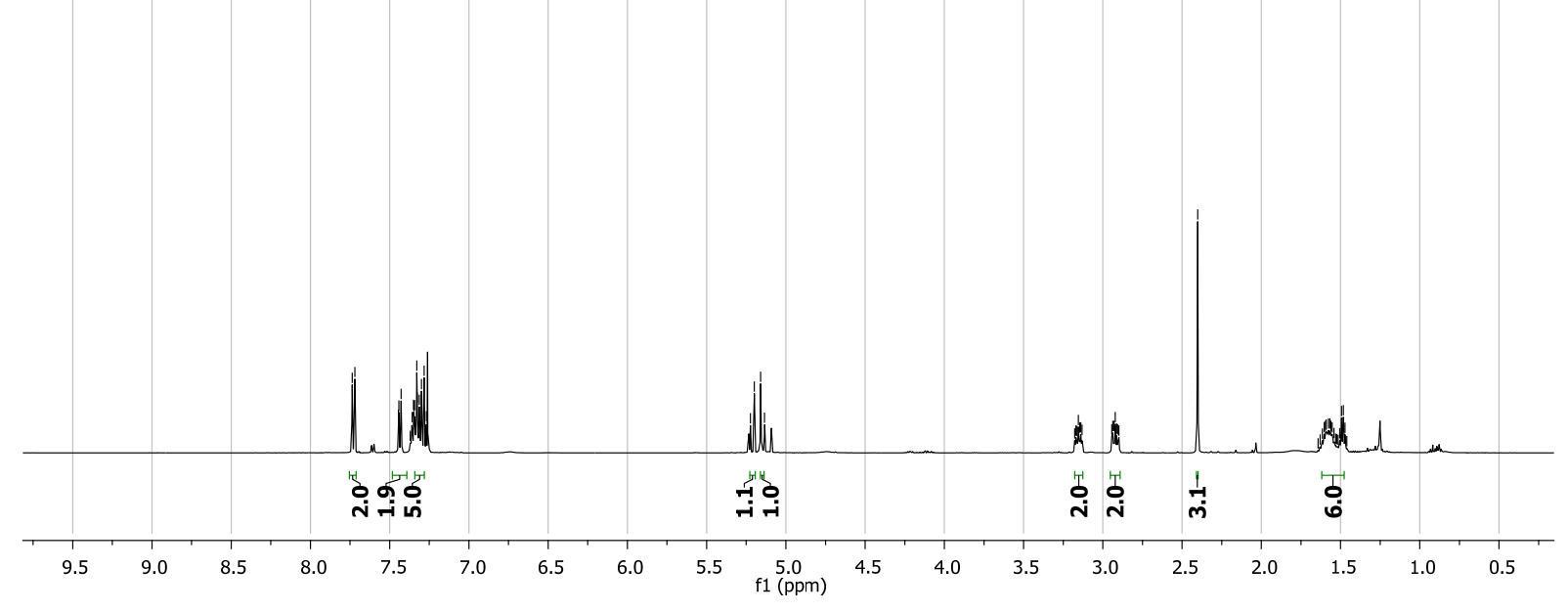




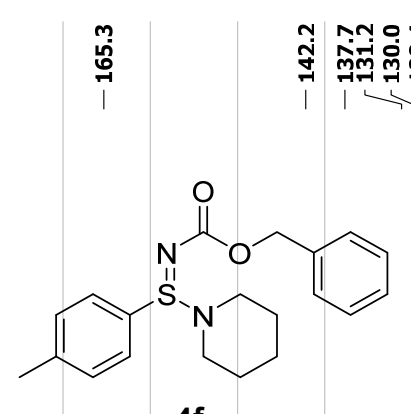

${ }^{13} \mathrm{C} \mathrm{NMR}, \mathrm{CDCl}_{3}, 125 \mathrm{MHz}$
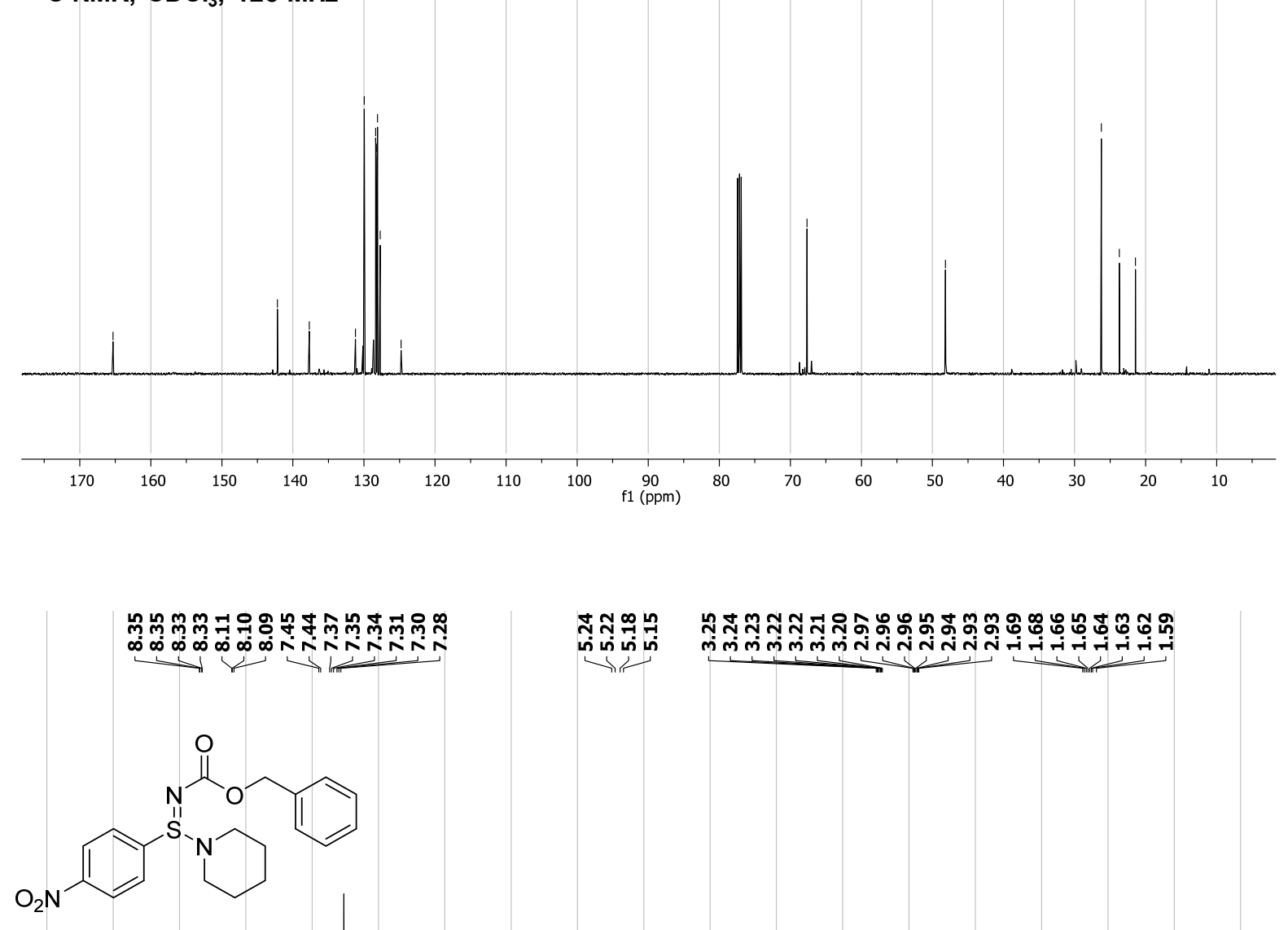

${ }^{1} \mathrm{H} \mathrm{NMR}, \stackrel{4 \mathrm{~g}}{\mathrm{CDCl}_{3}, 500 \mathrm{MHz}}$

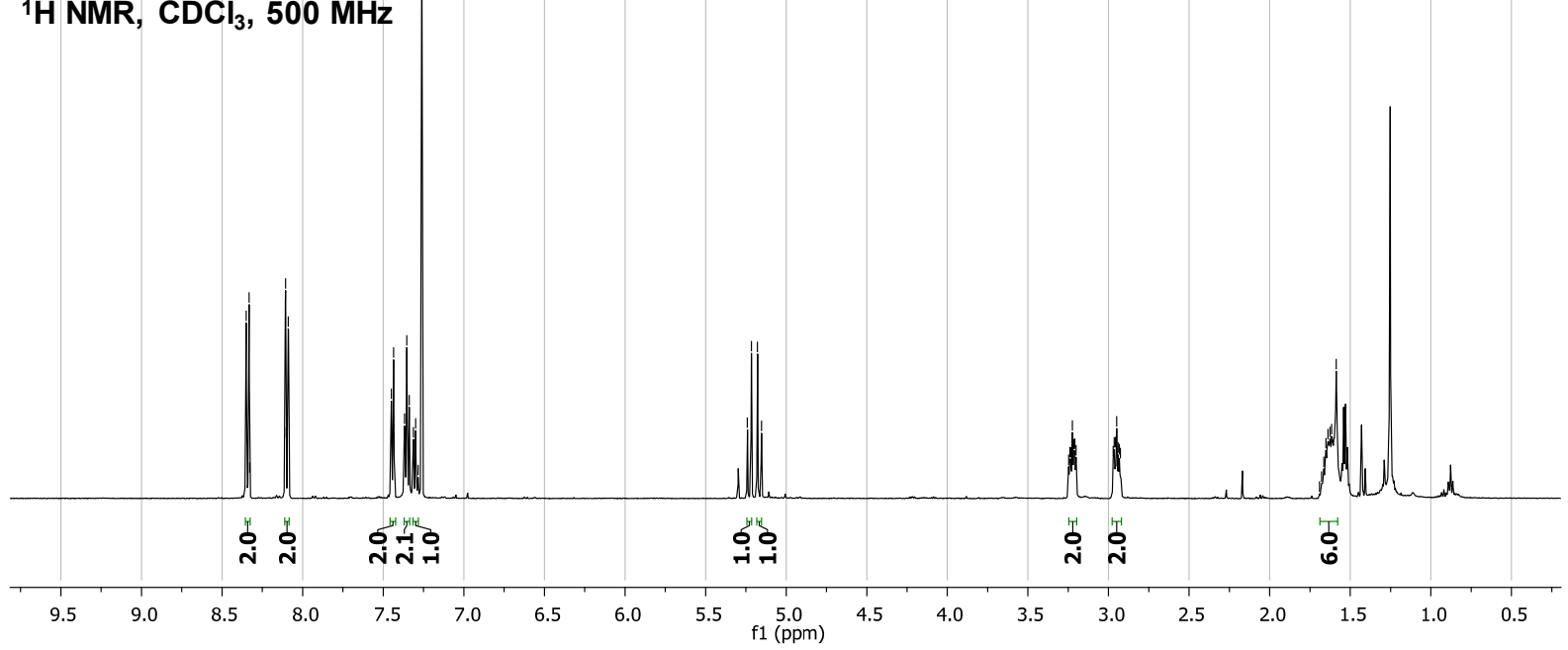



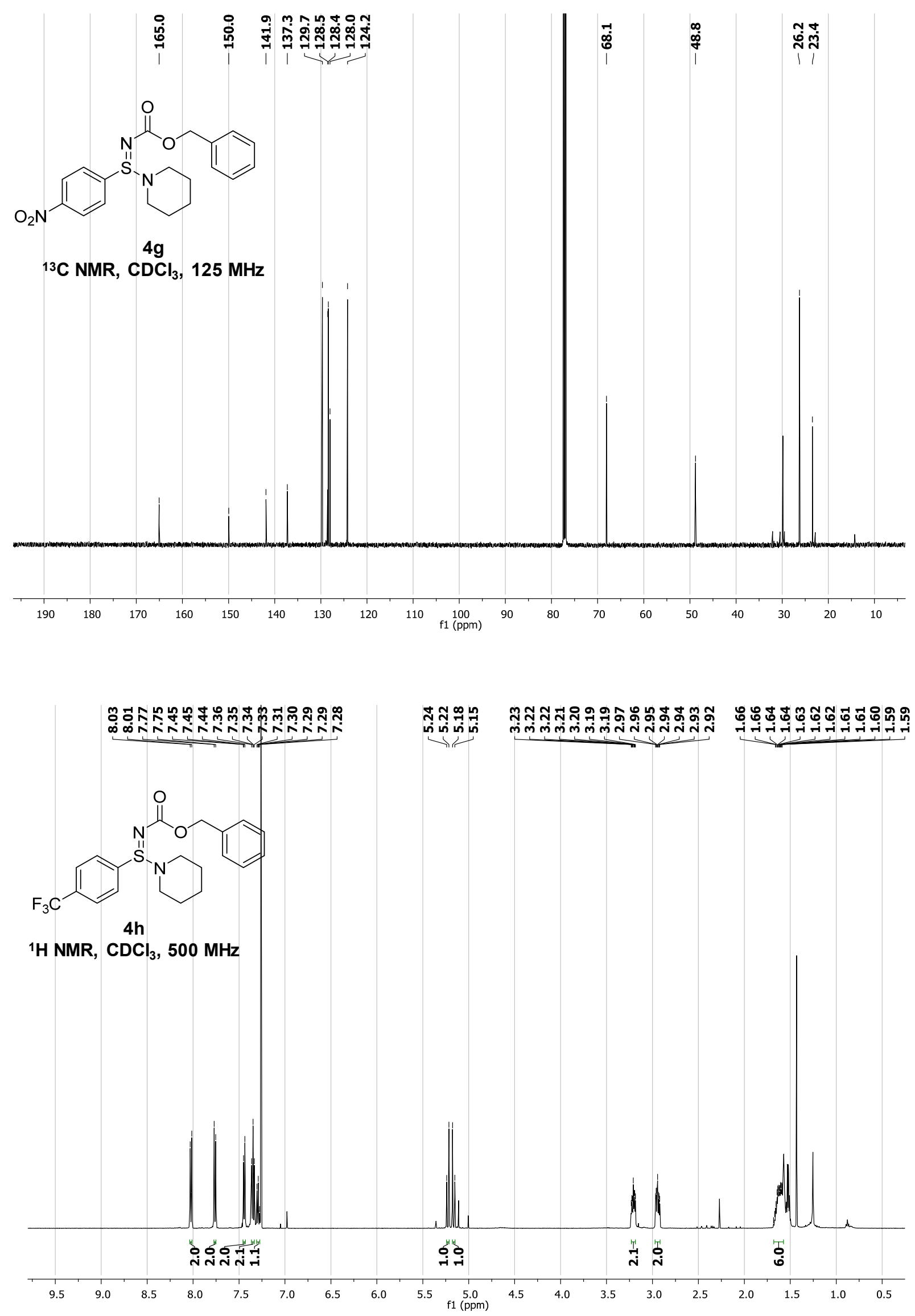

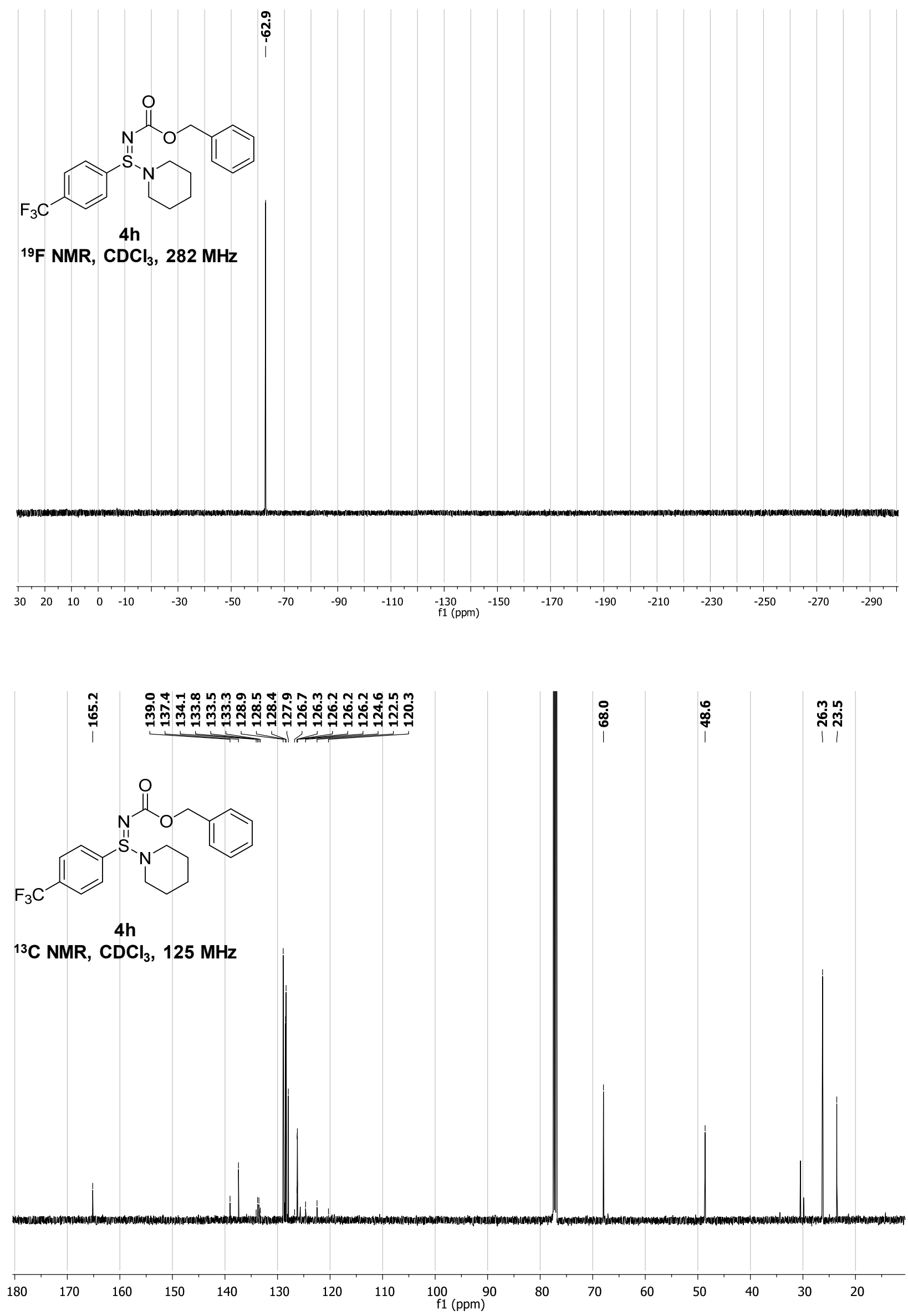


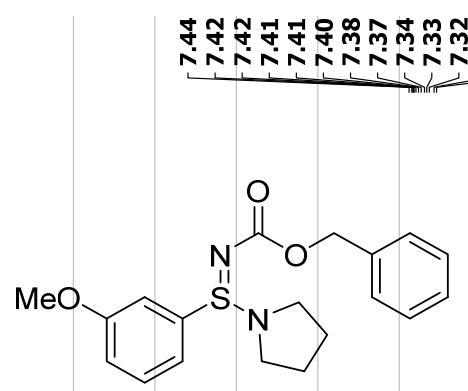

$4 i$

${ }^{1} \mathrm{H}$ NMR, $\mathrm{CDCl}_{3}, 500 \mathrm{MHz}$

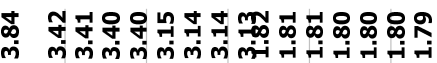

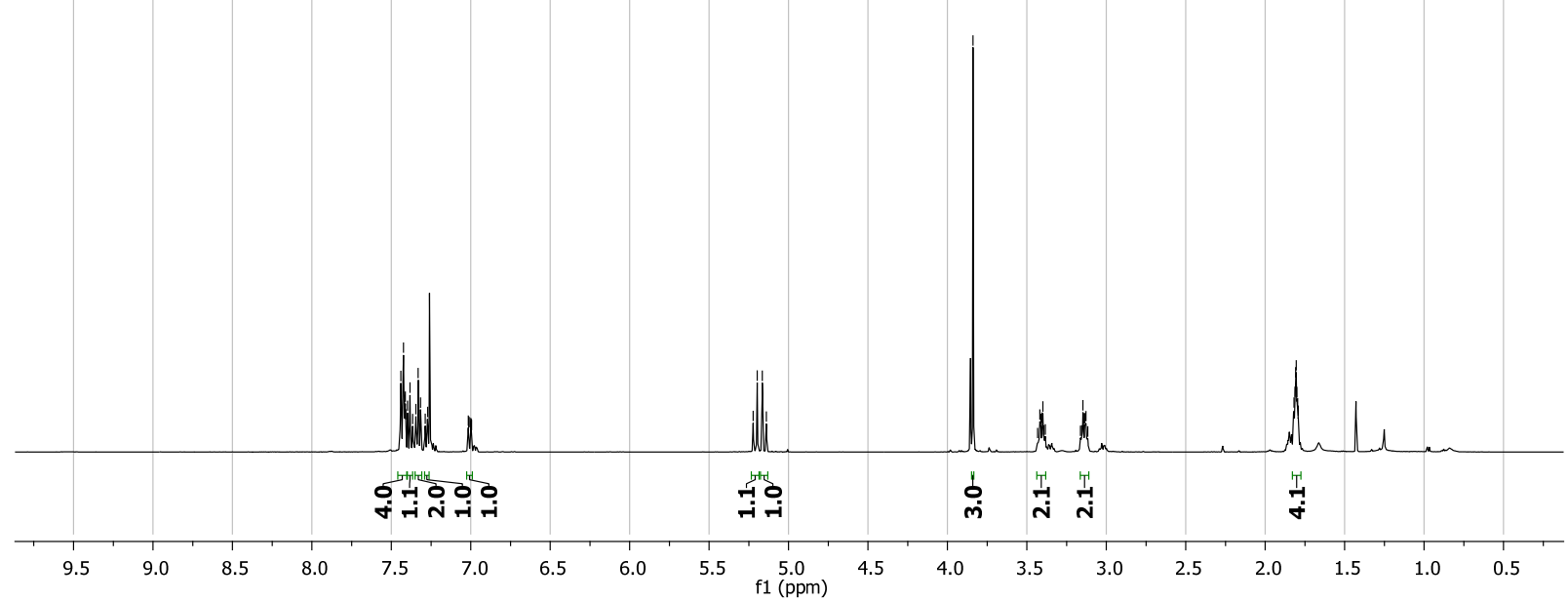

นุำ

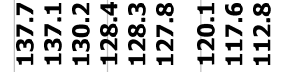<smiles>COc1cccc([SH](N=C(OCc2ccccc2)N2CCCC2)N2CCCC2)c1</smiles>

$4 i$

${ }^{13} \mathrm{C} \mathrm{NMR,} \mathrm{CDCl}_{3}, 125 \mathrm{MHz}$

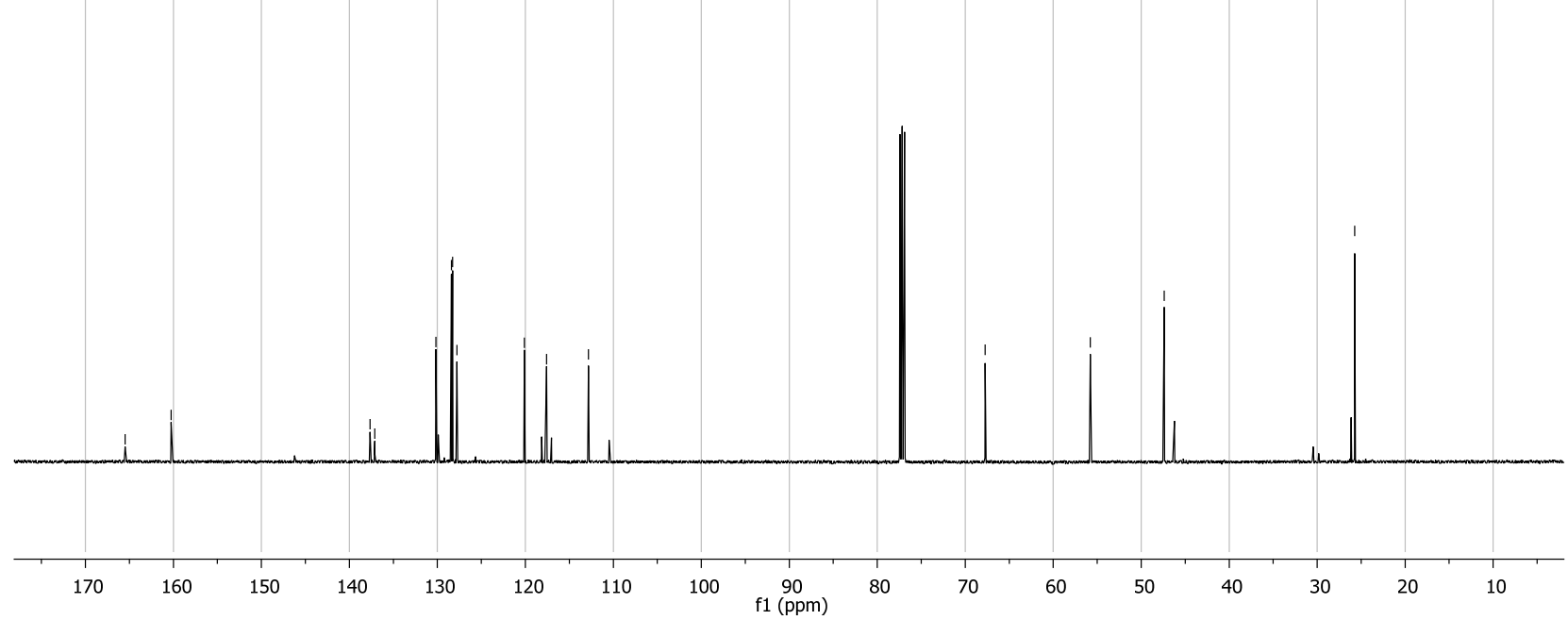




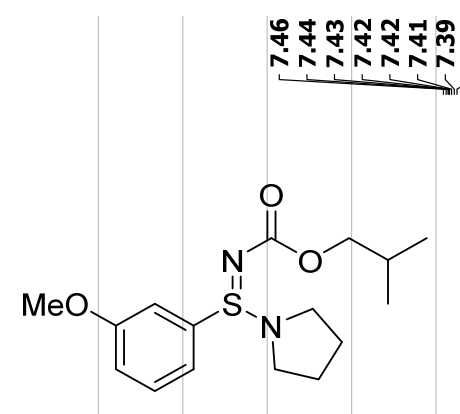

${ }^{1} \mathrm{H} \mathrm{NMR}, \stackrel{4 \mathrm{j}}{\mathrm{CDCl}_{3}, 500 \mathrm{MHz}}$

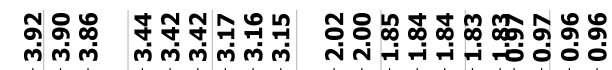
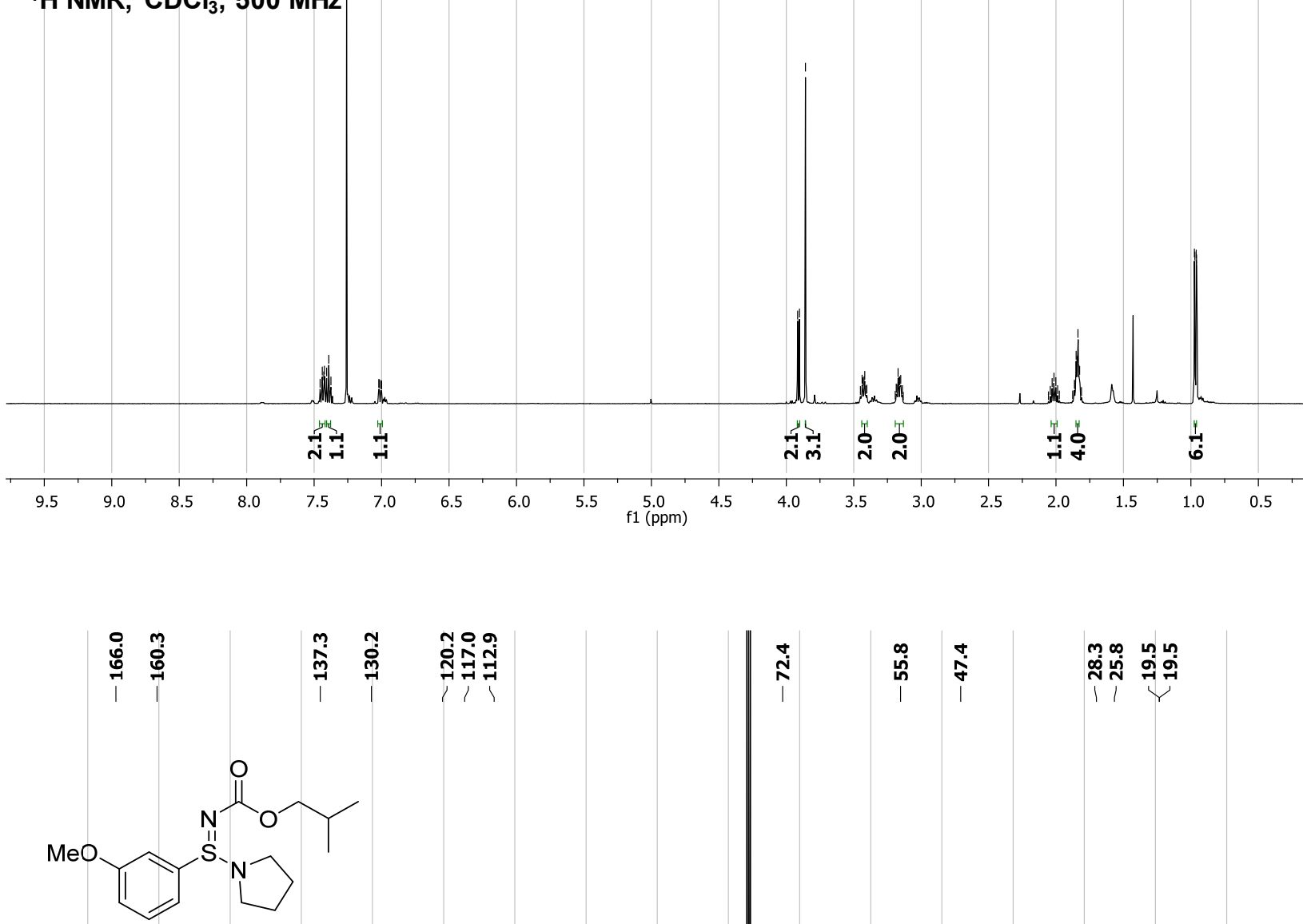

$4 \mathrm{j}$

${ }^{13} \mathrm{C} \mathrm{NMR,} \mathrm{CDCl}_{3}, 125 \mathrm{MHz}$

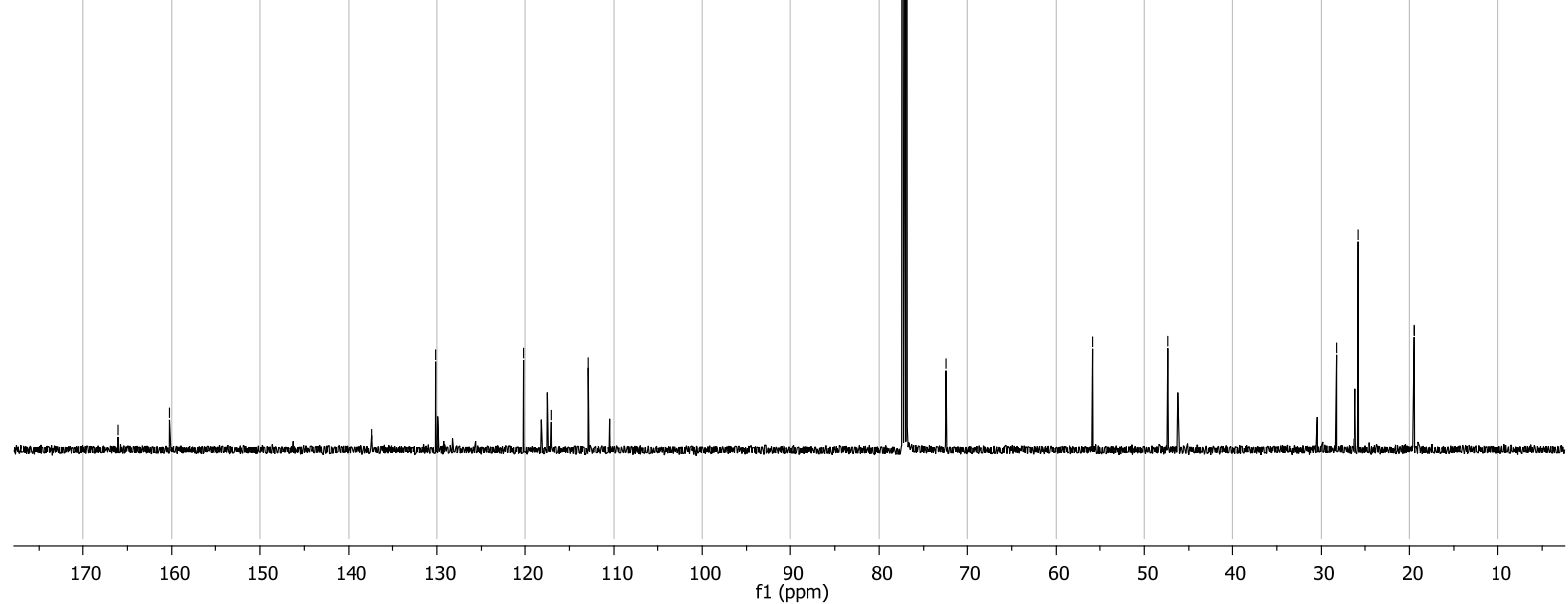



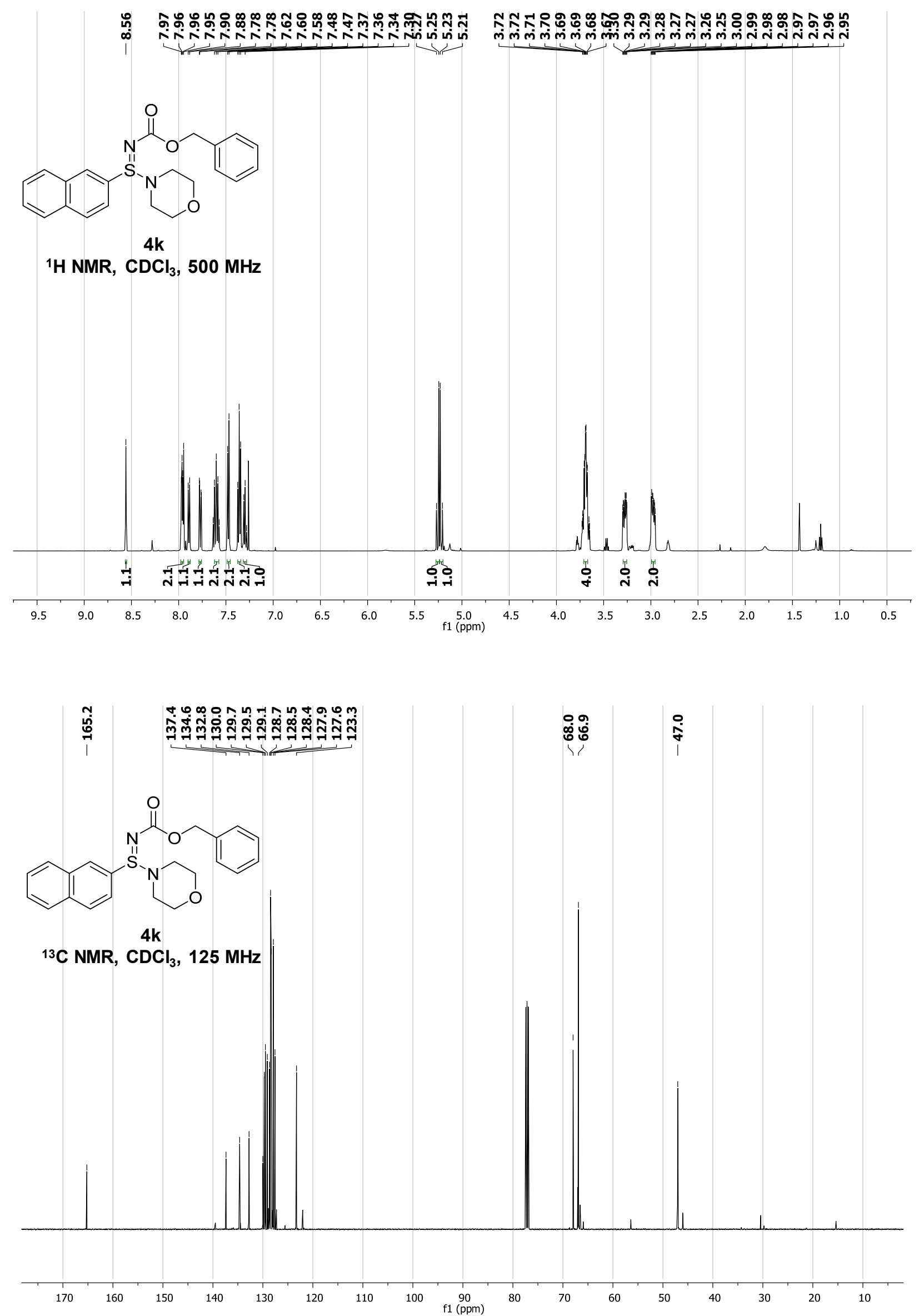

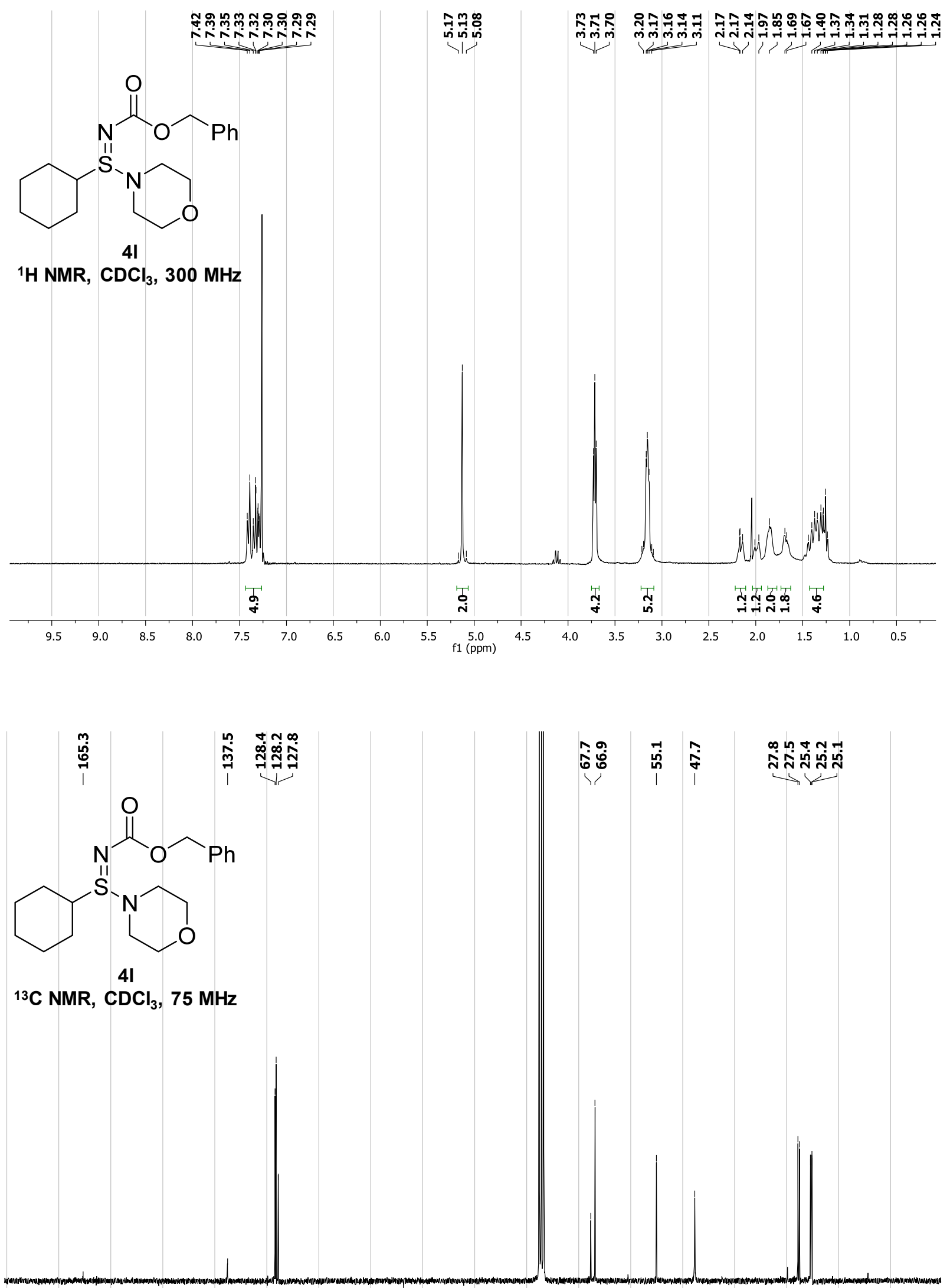

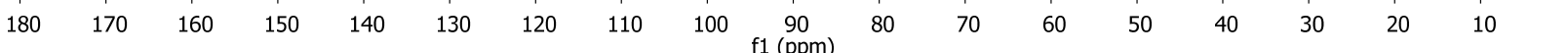



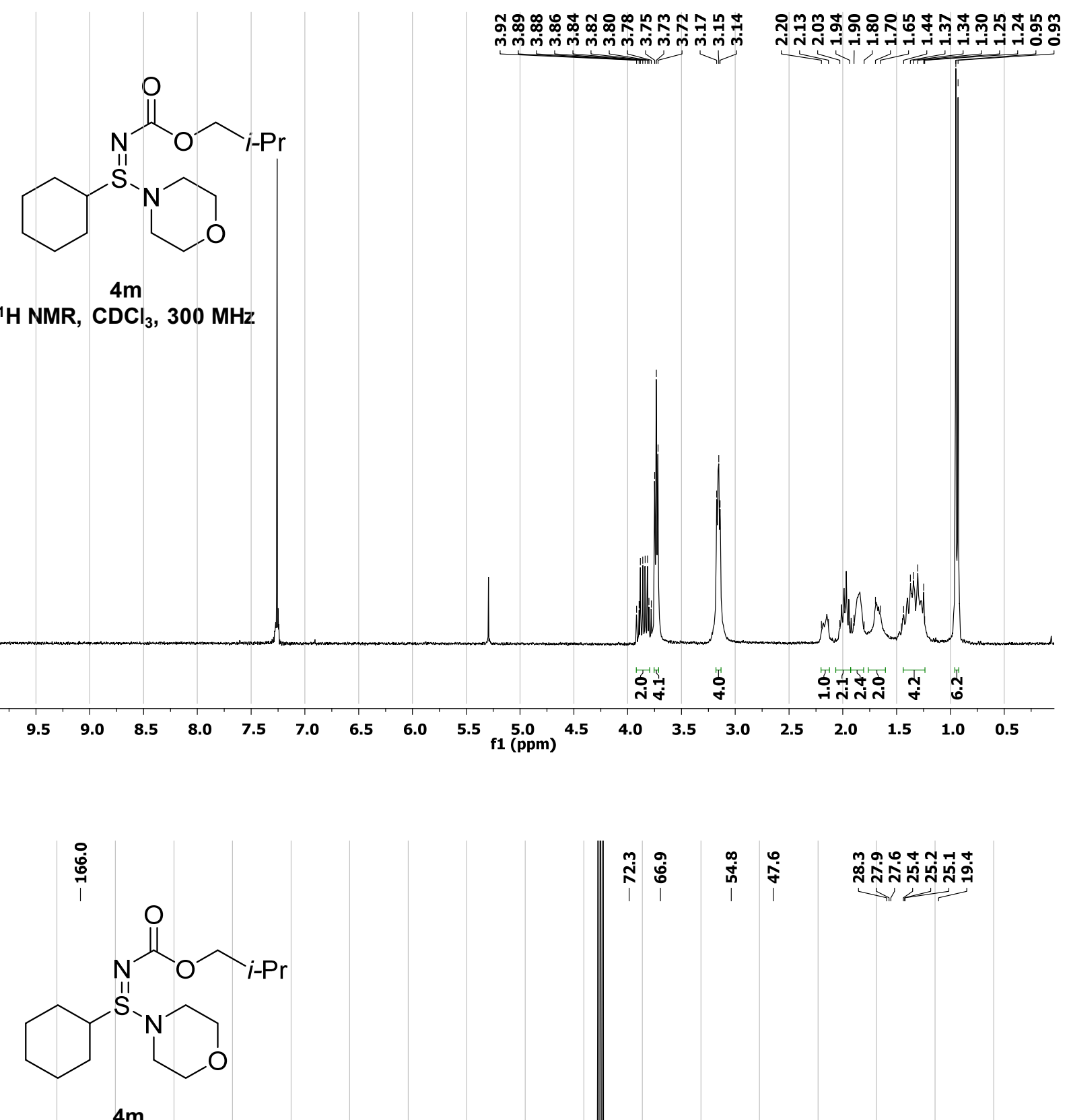

${ }^{13} \mathrm{C} \mathrm{NMR}, \mathrm{CDCl}_{3}, 75 \mathrm{MHz}$

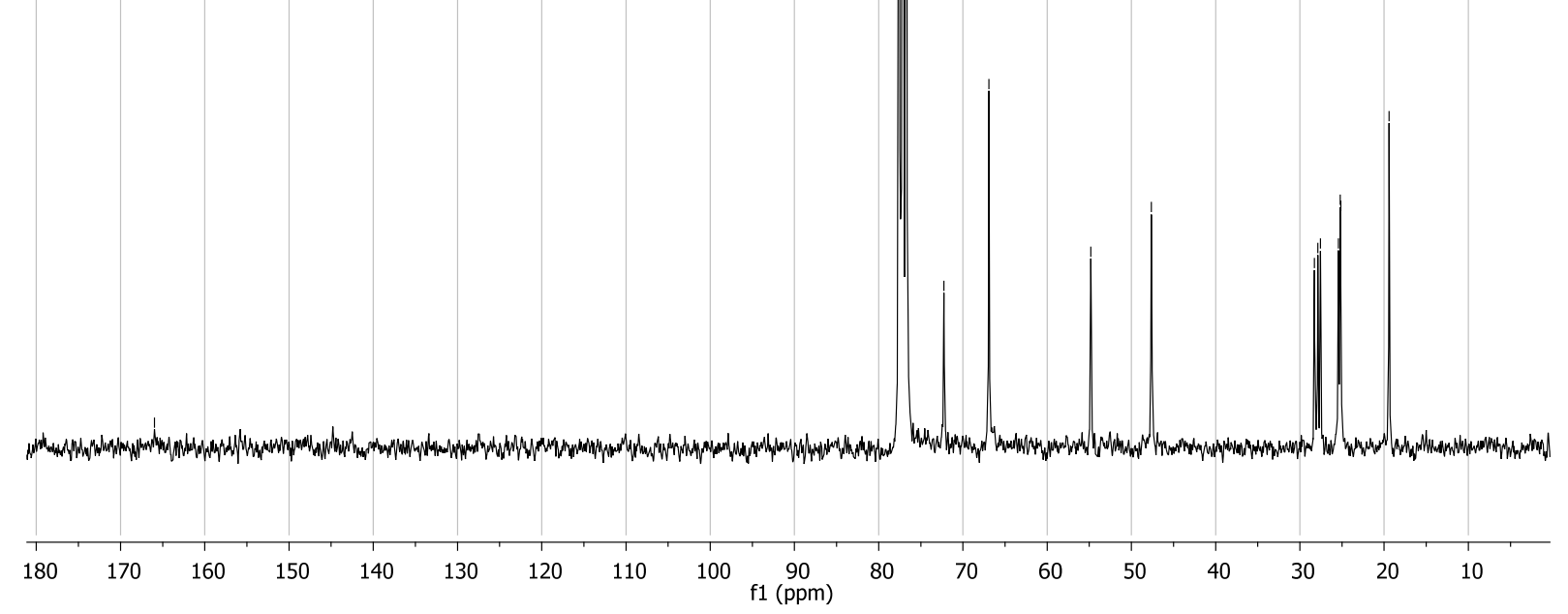




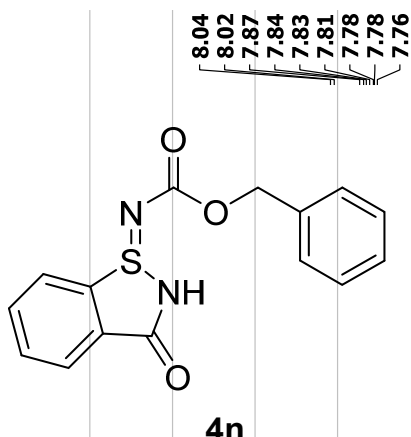

${ }^{1} \mathrm{H}$ NMR, $\mathrm{CDCl}_{3}, 300 \mathrm{MHz}$
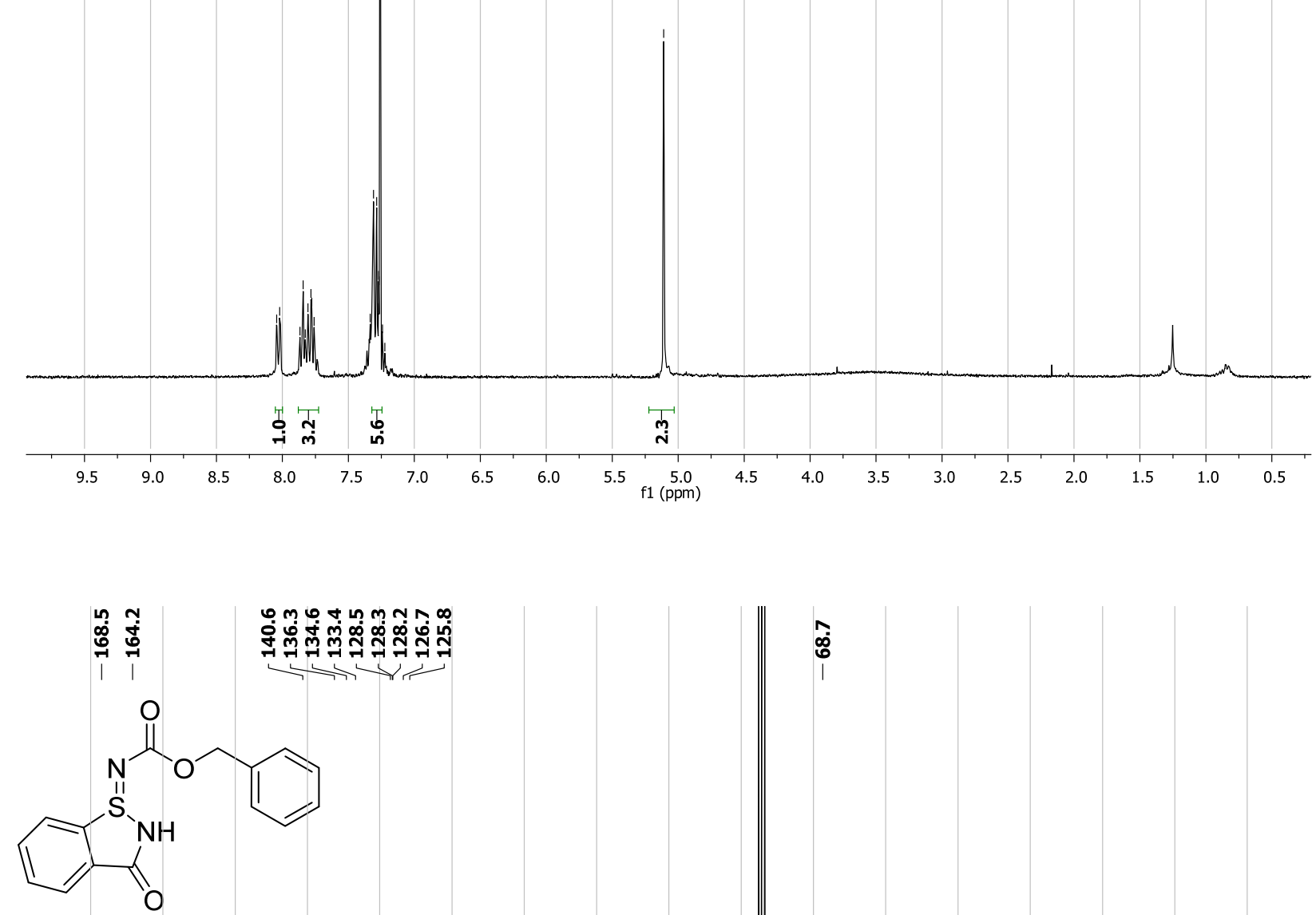

4n

${ }^{13} \mathrm{C} \mathrm{NMR,} \mathrm{CDCl}_{3}, 75 \mathrm{NHz}$

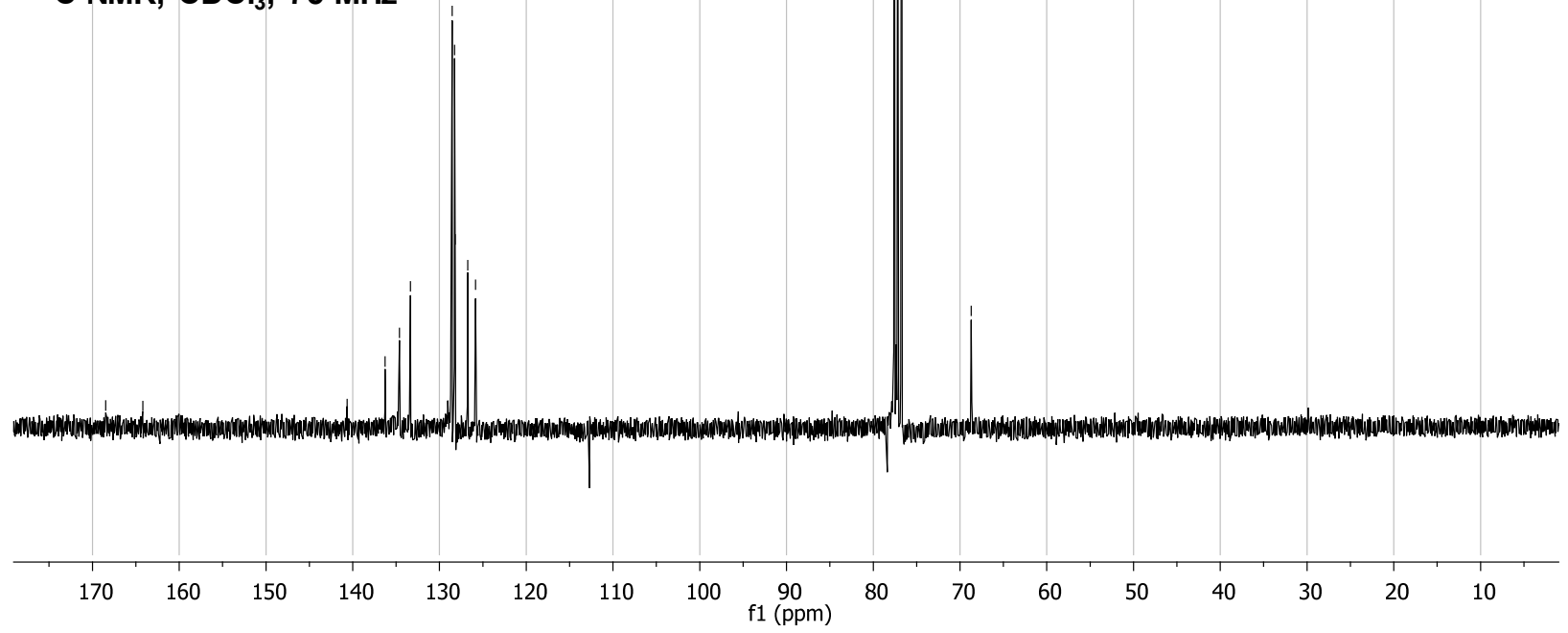




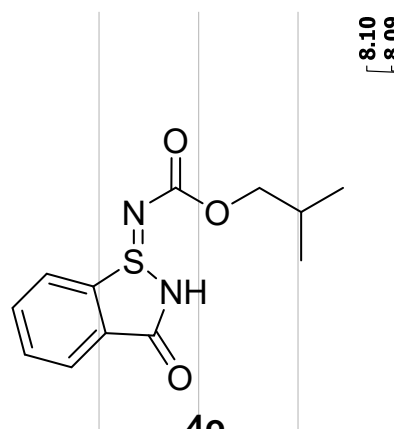

${ }^{1} \mathrm{H} \mathrm{NMR}, \mathrm{CDCl}_{3}, 300 \mathrm{MHz}$
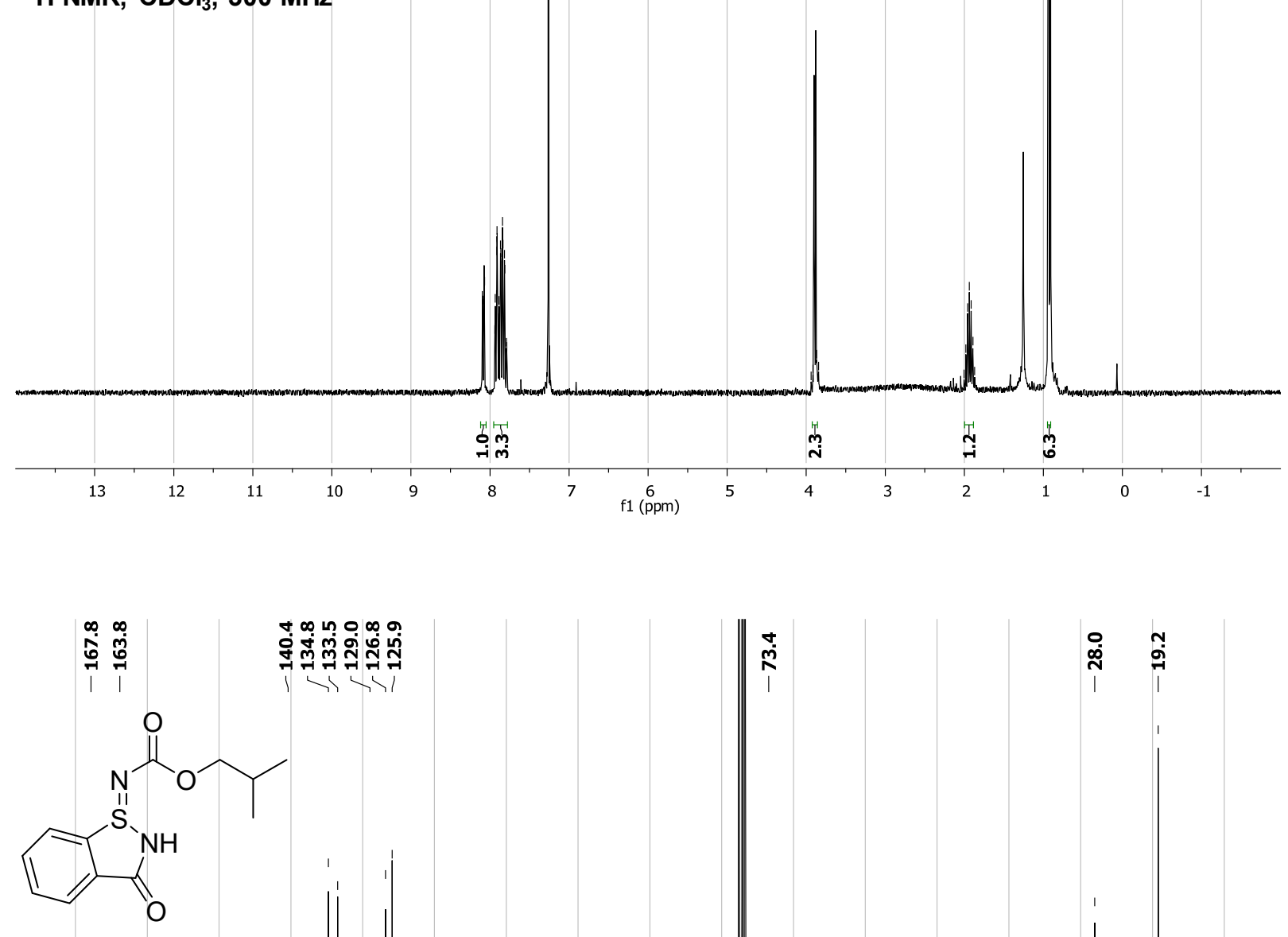

40

${ }^{13} \mathrm{C} \mathrm{NMR}, \mathrm{CDCl}_{3}, 75 \mathrm{MHz}$

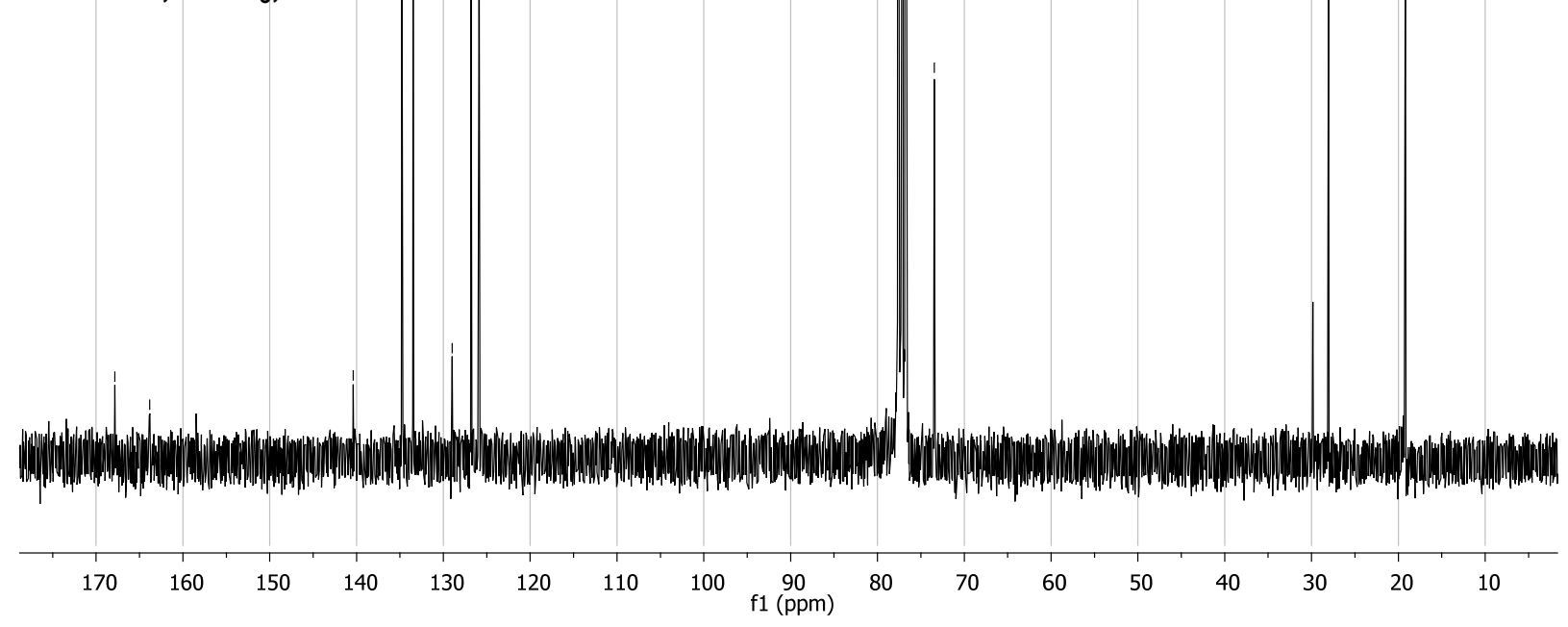




\section{X-Ray analysis for compounds $3 \mathrm{j}$ and 4}

\section{Methyl $N-(($ benzyloxy)carbonyl)-4-fluorophenylsulfinimidate (3j)}

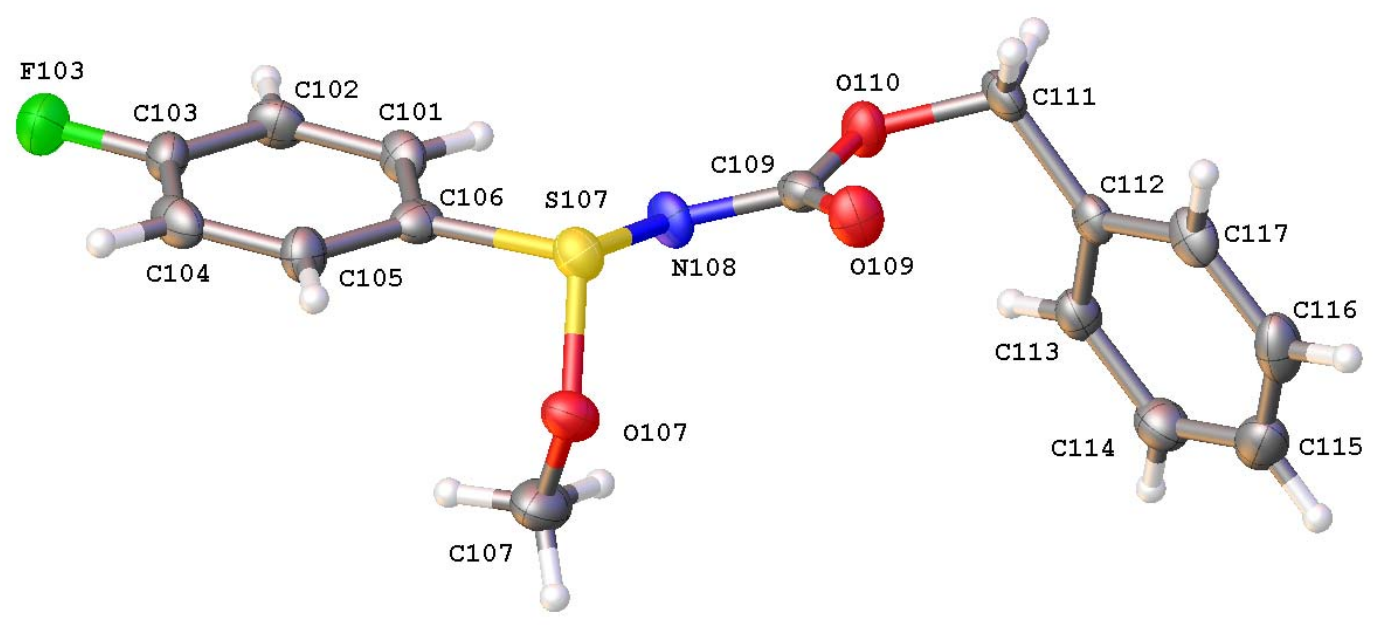

solid state structure of $3 \mathbf{j}$ with atom labelling and thermal ellipsoids drawn at $50 \%$ probability level

\section{Crystal structure determination of $\mathbf{3 j}$}

The asymmetric unit contains 2 chemically identical but crystallographically independent molecules, there are 4 molecules in the unit cell related by an inversion centre. Nothing unusual to report in the refinement or crystal packing.

\section{Experimental}

Single crystals of $\mathrm{C}_{15} \mathrm{H}_{14} \mathrm{FNO}_{3} \mathrm{~S} 3 \mathbf{j}$ were grown from $\mathrm{Et}_{2} \mathrm{O}$. A suitable crystal was selected and mounted on a glass fibre with Fomblin oil and placed on a Rigaku Oxford Diffraction SuperNova diffractometer with a dual source (Cu at zero) equipped with an AtlasS2 CCD area detector. The crystal was kept at 150(2) K during data collection. The structure was solved with ShelXT (2) structure solution program using the Intrinsic Phasing solution method and by using Olex2 (1) as the graphical interface. The model was refined with version 2018/3 of ShelXL (3) using Least Squares minimization.

1. Dolomanov, O.V., Bourhis, L.J., Gildea, R.J, Howard, J.A.K. \& Puschmann, H. (2009), J. Appl. Cryst. 42, 339-341.

2. Sheldrick, G.M., Acta Cryst., 2015, A71, 3-8.

3. Sheldrick, G.M. (2015). Acta Cryst. C71, 3-8.

Crystal Data for $\mathrm{C}_{15} \mathrm{H}_{14} \mathrm{FNO}_{3} \mathrm{~S}(M=307.33 \mathrm{~g} / \mathrm{mol})$ : triclinic, space group P-1 (no. 2), $a=10.69161(15) \AA, b=11.63326(15) \AA, c=$ 13.18188(18) $\AA, \alpha=109.8660(12)^{\circ}, \beta=110.4670(13)^{\circ}, \gamma=91.5851(11)^{\circ}, V=1424.53(4) \AA^{3}, Z=4, T=150(2) \mathrm{K}, \mu(\mathrm{CuK \alpha})=2.221 \mathrm{~mm}$ ${ }^{1}$, Dcalc $=1.433 \mathrm{~g} / \mathrm{cm}^{3}, 53586$ reflections measured $\left(7.716^{\circ} \leq 2 \Theta \leq 147.33^{\circ}\right), 5702$ unique $\left(R_{\text {int }}=0.0318, R_{\text {sigma }}=0.0140\right)$ which were used in all calculations. The final $R_{1}$ was $0.0308(\mathrm{I}>2 \sigma(\mathrm{I}))$ and $w R_{2}$ was 0.0839 (all data).

\section{Table 1 Crystal data and structure refinement for $3 \mathbf{j}$}

$\begin{array}{ll}\text { Identification code } & \mathrm{rl} 10 \\ \text { Empirical formula } & \mathrm{C}_{15} \mathrm{H}_{14} \mathrm{FNO}_{3} \mathrm{~S} \\ \text { Formula weight } & 307.33 \\ \text { Temperature/K } & 150(2)\end{array}$




\begin{tabular}{|c|c|}
\hline Crystal system & triclinic \\
\hline Space group & $\mathrm{P}-1$ \\
\hline$a / \AA$ & $10.69161(15)$ \\
\hline $\mathrm{b} / \AA \AA$ & $11.63326(15)$ \\
\hline$c / \AA$ & 13.18188(18) \\
\hline$\alpha /^{\circ}$ & $109.8660(12)$ \\
\hline$\beta /^{\circ}$ & $110.4670(13)$ \\
\hline $\mathrm{y} /{ }^{\circ}$ & $91.5851(11)$ \\
\hline Volume $/ \AA^{3}$ & $1424.53(4)$ \\
\hline Z & 4 \\
\hline$\rho_{\text {calc }} \mathrm{g} / \mathrm{cm}^{3}$ & 1.433 \\
\hline$\mu / \mathrm{mm}^{-1}$ & 2.221 \\
\hline$F(000)$ & 640.0 \\
\hline Crystal size $/ \mathrm{mm}^{3}$ & $0.35 \times 0.2 \times 0.16$ \\
\hline Radiation & $\operatorname{CuKa}(\lambda=1.54184)$ \\
\hline \multicolumn{2}{|c|}{$2 \Theta$ range for data collection $/{ }^{\circ} 7.716$ to 147.33} \\
\hline Index ranges & $-13 \leq h \leq 13,-14 \leq k \leq 14,-16 \leq \mathrm{I} \leq 16$ \\
\hline Reflections collected & 53586 \\
\hline Independent reflections & $5702\left[R_{\text {int }}=0.0318, R_{\text {sigma }}=0.0140\right]$ \\
\hline Data/restraints/parameters & $5702 / 0 / 381$ \\
\hline Goodness-of-fit on $F^{2}$ & 1.047 \\
\hline Final $R$ indexes $[\mid>=2 \sigma(I)]$ & $\mathrm{R}_{1}=0.0308, \mathrm{wR}_{2}=0.0833$ \\
\hline Final $\mathrm{R}$ indexes [all data] & $\mathrm{R}_{1}=0.0316, \mathrm{wR}_{2}=0.0839$ \\
\hline Largest diff. peak/hole / e $\AA^{-3}$ & $0.43 /-0.37$ \\
\hline
\end{tabular}

Table 2 Fractional Atomic Coordinates $\left(\times 10^{4}\right)$ and Equivalent Isotropic Displacement Parameters $\left(\AA^{2} \times 10^{3}\right)$ for $3 j$. $U_{\text {eq }}$ is defined as $1 / 3$ of of the trace of the orthogonalised $U_{I J}$ tensor.

$\begin{array}{lrrrr}\text { Atom } & \boldsymbol{x} & \boldsymbol{y} & \boldsymbol{z} & \mathrm{U}(\mathrm{eq}) \\ \mathrm{S} 107 & 4211.6(3) & -574.6(3) & 2738.1(3) & 25.30(9) \\ \mathrm{F} 103 & 2432.7(10) & -5392.8(8) & -1128.3(8) & 42.2(2) \\ \mathrm{O} 107 & 4608.3(10) & 344.5(9) & 2166.7(9) & 31.9(2) \\ \text { O109 } & 4156.6(10) & 1166.0(9) & 4780.2(9) & 31.8(2) \\ \text { O110 } & 1984.0(9) & 381.3(9) & 4296.1(9) & 28.3(2) \\ \text { N108 } & 2851.6(11) & -336.7(11) & 2949.1(10) & 27.6(2) \\ \text { C101 } & 2331.9(13) & -2672.2(13) & 1173.4(12) & 28.3(3)\end{array}$




\begin{tabular}{|c|c|c|c|c|}
\hline C102 & $1934.6(14)$ & $-3818.0(13)$ & $274.3(13)$ & $30.9(3)$ \\
\hline C103 & $2825.3(15)$ & $-4272.1(13)$ & $-253.0(12)$ & $30.1(3)$ \\
\hline C104 & $4092.7(14)$ & $-3620.5(13)$ & $71.5(13)$ & $30.6(3)$ \\
\hline C105 & $4484.0(13)$ & $-2471.1(13)$ & $966.9(12)$ & $28.5(3)$ \\
\hline C106 & $3602.4(13)$ & $-2005.3(12)$ & $1514.1(12)$ & $25.0(3)$ \\
\hline C107 & $3572.9(17)$ & $467.3(15)$ & $1161.2(14)$ & $39.6(3)$ \\
\hline C109 & $3117.4(13)$ & $466.8(12)$ & $4071.3(12)$ & $25.0(3)$ \\
\hline C111 & $2094.7(15)$ & $1202.2(13)$ & $5440.2(12)$ & $29.5(3)$ \\
\hline C112 & $2090.0(13)$ & 2526.6(12) & $5534.6(12)$ & $25.8(3)$ \\
\hline C113 & $1162.6(14)$ & $2808.2(13)$ & $4641.6(12)$ & $28.3(3)$ \\
\hline C114 & $1135.6(16)$ & $4026.2(14)$ & $4736.9(14)$ & $34.0(3)$ \\
\hline C115 & 2023.3(16) & $4980.0(14)$ & $5722.9(15)$ & $37.1(3)$ \\
\hline C116 & $2937.9(15)$ & $4710.0(14)$ & $6617.4(14)$ & $36.9(3)$ \\
\hline C117 & $2975.4(14)$ & $3485.0(14)$ & $6521.0(12)$ & $31.7(3)$ \\
\hline S207 & $6804.6(3)$ & $5545.8(3)$ & $8041.3(3)$ & $25.20(9)$ \\
\hline F203 & $8837.6(9)$ & $1712.8(8)$ & $5111.5(8)$ & $39.0(2)$ \\
\hline O207 & $5916.1(10)$ & $6183.7(10)$ & $7170.4(9)$ & $32.7(2)$ \\
\hline O209 & $6891.1(9)$ & $7386.4(9)$ & $10067.4(9)$ & $30.4(2)$ \\
\hline $\mathrm{O} 210$ & $9178.9(9)$ & $7753.2(9)$ & $10820.8(9)$ & $29.7(2)$ \\
\hline N208 & $8166.0(11)$ & $6455.3(10)$ & $8983.4(10)$ & $26.8(2)$ \\
\hline C201 & $6560.3(14)$ & $3431.4(13)$ & $6246.3(13)$ & $30.0(3)$ \\
\hline C202 & $7020.4(15)$ & $2505.5(13)$ & $5553.0(13)$ & $32.6(3)$ \\
\hline C203 & $8387.5(14)$ & 2625.3(12) & $5780.9(13)$ & $28.9(3)$ \\
\hline C204 & $9316.4(14)$ & $3627.3(13)$ & $6661.6(13)$ & $29.3(3)$ \\
\hline C205 & $8850.4(13)$ & $4555.1(13)$ & $7352.8(12)$ & $27.9(3)$ \\
\hline C206 & $7474.7(13)$ & $4452.1(12)$ & $7138.8(12)$ & $25.3(3)$ \\
\hline C207 & $6566.3(17)$ & $6708.7(15)$ & $6590.7(15)$ & $38.9(3)$ \\
\hline C209 & $7960.7(13)$ & $7202.8(12)$ & $9955.3(12)$ & $24.8(3)$ \\
\hline C211 & $9138.6(15)$ & $8623.5(13)$ & $11895.6(12)$ & $30.0(3)$ \\
\hline C212 & $8846.9(13)$ & $9856.5(12)$ & $11815.7(11)$ & $25.8(3)$ \\
\hline C213 & $9603.8(14)$ & $10475.0(14)$ & $11429.2(13)$ & $31.5(3)$ \\
\hline C214 & $9374.6(15)$ & $11635.0(15)$ & $11398.1(13)$ & $36.0(3)$ \\
\hline C215 & 8399.6(16) & $12189.0(14)$ & $11769.3(13)$ & $36.1(3)$ \\
\hline C216 & 7642.1(15) & 11582.1(14) & $12153.5(13)$ & $34.8(3)$ \\
\hline C217 & $7864.2(14)$ & 10416.1(13) & $12170.6(12)$ & $29.3(3)$ \\
\hline
\end{tabular}


Table 3 Anisotropic Displacement Parameters $\left(\AA^{2} \times 10^{3}\right)$ for 3j. The Anisotropic displacement factor exponent takes the form: $2 \pi^{2}\left[h^{2} a^{\star 2} U_{11}+2 h k a^{*} b^{*} U_{12}+\ldots\right]$.

\begin{tabular}{|c|c|c|c|c|c|c|}
\hline Atom & $U_{11}$ & $U_{22}$ & $\mathbf{U}_{33}$ & $\mathbf{U}_{23}$ & $U_{13}$ & $U_{12}$ \\
\hline S107 & $19.71(15)$ & $29.32(17)$ & $26.56(16)$ & $9.35(13)$ & $9.43(12)$ & $5.30(12)$ \\
\hline F103 & $55.6(6)$ & $28.2(4)$ & $39.2(5)$ & $5.3(4)$ & $21.4(4)$ & $1.9(4)$ \\
\hline O107 & 29.1(5) & $32.4(5)$ & $33.4(5)$ & $11.2(4)$ & $12.6(4)$ & $-0.4(4)$ \\
\hline O109 & $25.0(5)$ & $33.2(5)$ & $32.2(5)$ & $6.4(4)$ & $10.9(4)$ & $1.8(4)$ \\
\hline 0110 & 27.1(5) & $26.3(5)$ & $32.7(5)$ & $6.8(4)$ & $16.8(4)$ & $3.6(4)$ \\
\hline N108 & $22.0(5)$ & $30.9(6)$ & $30.4(6)$ & $8.9(5)$ & $12.9(5)$ & $6.2(4)$ \\
\hline C101 & $24.8(6)$ & $32.7(7)$ & $32.9(7)$ & $14.3(6)$ & 15.1(6) & $7.0(5)$ \\
\hline C102 & $28.9(7)$ & $32.2(7)$ & $33.6(7)$ & $15.1(6)$ & $11.9(6)$ & $0.4(6)$ \\
\hline C103 & $38.7(8)$ & 25.1(7) & $27.8(7)$ & $11.2(6)$ & $12.9(6)$ & $6.6(6)$ \\
\hline C104 & $30.9(7)$ & $32.9(7)$ & $34.3(7)$ & $14.3(6)$ & $17.5(6)$ & $12.4(6)$ \\
\hline C105 & 23.1(6) & $31.5(7)$ & $33.0(7)$ & $12.2(6)$ & $12.8(6)$ & $6.7(5)$ \\
\hline C106 & $23.9(6)$ & $25.4(6)$ & $26.7(6)$ & $10.2(5)$ & $10.4(5)$ & $6.0(5)$ \\
\hline C107 & 48.6(9) & $35.9(8)$ & $34.7(8)$ & $16.8(7)$ & $12.9(7)$ & $5.9(7)$ \\
\hline C109 & $24.8(6)$ & $24.4(6)$ & $30.1(7)$ & $12.6(5)$ & $12.9(5)$ & $6.8(5)$ \\
\hline C111 & $33.3(7)$ & $31.8(7)$ & $30.0(7)$ & $12.3(6)$ & $18.6(6)$ & $8.7(6)$ \\
\hline C112 & $24.9(6)$ & $28.7(7)$ & $27.6(7)$ & $8.7(6)$ & $16.0(5)$ & $6.1(5)$ \\
\hline C113 & $27.5(6)$ & $29.7(7)$ & $27.8(7)$ & $8.4(6)$ & $13.0(5)$ & $3.5(5)$ \\
\hline C114 & $37.0(8)$ & $35.5(8)$ & $37.0(8)$ & 17.1(7) & $19.0(6)$ & $11.5(6)$ \\
\hline C115 & $43.2(8)$ & $26.8(7)$ & 48.9(9) & $12.4(7)$ & $28.0(7)$ & $7.0(6)$ \\
\hline C116 & $31.1(7)$ & $32.9(8)$ & $38.6(8)$ & $0.0(6)$ & $17.3(6)$ & $-1.0(6)$ \\
\hline C117 & $25.8(7)$ & $37.4(8)$ & 28.6(7) & $7.1(6)$ & $11.5(6)$ & $6.3(6)$ \\
\hline S207 & $21.09(15)$ & $25.21(16)$ & $29.80(17)$ & $8.96(13)$ & $11.72(13)$ & $1.73(12)$ \\
\hline F203 & $43.2(5)$ & $26.8(4)$ & $48.7(5)$ & $5.5(4)$ & $28.0(4)$ & $4.0(4)$ \\
\hline O207 & $27.2(5)$ & $36.6(5)$ & $37.2(5)$ & $16.1(5)$ & $13.3(4)$ & $9.3(4)$ \\
\hline O209 & $24.3(5)$ & $33.2(5)$ & $35.5(5)$ & $10.3(4)$ & $15.7(4)$ & $4.7(4)$ \\
\hline $\mathrm{O} 210$ & $23.0(4)$ & $29.2(5)$ & $30.9(5)$ & $3.9(4)$ & $10.6(4)$ & $3.7(4)$ \\
\hline N208 & $23.0(5)$ & $25.1(6)$ & $30.6(6)$ & $6.2(5)$ & $12.7(5)$ & $-0.2(4)$ \\
\hline C201 & $23.6(6)$ & $28.9(7)$ & $33.8(7)$ & $8.3(6)$ & $10.3(6)$ & $-0.1(5)$ \\
\hline C202 & $30.4(7)$ & $26.7(7)$ & $34.1(7)$ & $5.3(6)$ & $11.2(6)$ & $-3.7(5)$ \\
\hline C203 & $34.6(7)$ & $23.3(6)$ & $33.8(7)$ & $10.7(6)$ & $18.7(6)$ & $5.2(5)$ \\
\hline C204 & $24.5(6)$ & $29.8(7)$ & $35.6(7)$ & $12.4(6)$ & $13.7(6)$ & $4.2(5)$ \\
\hline C205 & $23.8(6)$ & 26.2(7) & $30.8(7)$ & $8.1(6)$ & $9.6(5)$ & $-0.2(5)$ \\
\hline C206 & $24.4(6)$ & $24.3(6)$ & $28.7(7)$ & $10.5(5)$ & $11.3(5)$ & $3.2(5)$ \\
\hline C207 & $45.8(9)$ & $39.8(8)$ & $40.6(8)$ & $21.5(7)$ & $20.7(7)$ & $11.8(7)$ \\
\hline
\end{tabular}




$\begin{array}{lllllrr}\text { C209 } & 23.5(6) & 23.2(6) & 30.7(7) & 11.6(5) & 12.2(5) & 2.9(5) \\ \text { C211 } & 31.8(7) & 28.6(7) & 26.6(7) & 7.0(6) & 10.9(6) & 4.7(5) \\ \text { C212 } & 23.5(6) & 26.3(7) & 21.7(6) & 5.1(5) & 6.0(5) & -0.1(5) \\ \text { C213 } & 25.0(6) & 38.5(8) & 31.3(7) & 12.2(6) & 12.0(6) & 2.4(6) \\ \text { C214 } & 33.4(7) & 40.1(8) & 34.1(8) & 17.8(7) & 9.5(6) & -3.5(6) \\ \text { C215 } & 38.3(8) & 29.2(7) & 34.0(8) & 13.3(6) & 9.7(6) & 2.8(6) \\ \text { C216 } & 31.9(7) & 33.4(8) & 36.7(8) & 9.4(6) & 13.8(6) & 8.5(6) \\ \text { C217 } & 28.6(7) & 28.2(7) & 29.6(7) & 7.0(6) & 13.4(6) & 0.8(5)\end{array}$

Table 4 Bond Lengths for $\mathbf{3 j}$.

\begin{tabular}{lrrr} 
Atom Atom & Length/A & Atom Atom & \multicolumn{1}{c}{ Length/A } \\
S107 O107 & $1.6236(10)$ & S207 O207 & $1.6205(10)$ \\
S107 N108 & $1.5877(11)$ S207 N208 & $1.5931(11)$ \\
S107 C106 & $1.7825(14)$ & S207 C206 & $1.7790(14)$ \\
F103 C103 & $1.3475(17)$ & F203 C203 & $1.3512(16)$ \\
O107 C107 & $1.4539(18)$ & O207 C207 & $1.4510(18)$ \\
O109 C109 & $1.2152(17)$ & O209 C209 & $1.2188(16)$ \\
O110 C109 & $1.3530(15)$ & O210 C209 & $1.3514(16)$ \\
O110 C111 & $1.4459(17)$ & O210 C211 & $1.4506(17)$ \\
N108 C109 & $1.3763(18)$ & N208 C209 & $1.3768(18)$ \\
C101 C102 & $1.380(2)$ & C201 C202 & $1.383(2)$ \\
C101 C106 & $1.3882(19)$ & C201 C206 & $1.3899(19)$ \\
C102 C103 & $1.382(2)$ & C202 C203 & $1.378(2)$ \\
C103 C104 & $1.382(2)$ & C203 C204 & $1.379(2)$ \\
C104 C105 & $1.381(2)$ & C204 C205 & $1.386(2)$ \\
C105 C106 & $1.3930(18)$ & C205 C206 & $1.3909(18)$ \\
C111 C112 & $1.5034(19)$ & C211 C212 & $1.5068(19)$ \\
C112 C113 & $1.3927(19)$ & C212 C213 & $1.3919(19)$ \\
C112 C117 & $1.388(2)$ & C212 C217 & $1.3860(19)$ \\
C113 C114 & $1.381(2)$ & C213 C214 & $1.390(2)$ \\
C114 C115 & $1.386(2)$ & C214 C215 & $1.386(2)$ \\
C115 C116 & $1.381(2)$ & C215 C216 & $1.382(2)$ \\
C117 & $1.390(2)$ & C216 C217 & $1.390(2)$ \\
\hline
\end{tabular}


Table 5 Bond Angles for 3j.

\begin{tabular}{|c|c|c|c|}
\hline Atom Atom Atom & Angle $^{\circ}$ & Atom Atom Atom & Angle $/^{\circ}$ \\
\hline O107 S107 C106 & $101.44(6)$ & O207 S207 C206 & $102.24(6)$ \\
\hline N108 S107 O107 & $111.50(6)$ & N208 S207 O207 & $111.09(6)$ \\
\hline N108 S107 C106 & $99.61(6)$ & N208 S207 C206 & $99.99(6)$ \\
\hline C107 O107 S107 & $118.76(9)$ & C207 O207 S207 & $118.66(9)$ \\
\hline C109 O110 C111 & $114.86(10)$ & C209 O210 C211 & $115.51(10)$ \\
\hline C109 N108 S107 & 111.17(9) & C209 N208 S207 & $110.79(9)$ \\
\hline C102 C101 C106 & $119.34(13)$ & C202 C201 C206 & $119.65(13)$ \\
\hline C101 C102 C103 & $118.70(13)$ & C203 C202 C201 & $118.34(13)$ \\
\hline F103 C103 C102 & $118.41(13)$ & F203 C203 C202 & $118.27(13)$ \\
\hline F103 C103 C104 & $118.78(13)$ & F203 C203 C204 & $118.55(12)$ \\
\hline C102 C103 C104 & $122.80(13)$ & C202 C203 C204 & $123.18(13)$ \\
\hline C105 C104 C103 & $118.34(13)$ & C203 C204 C205 & $118.30(12)$ \\
\hline C104 C105 C106 & $119.54(13)$ & C204 C205 C206 & $119.48(13)$ \\
\hline C101 C106 S107 & $121.04(10)$ & C201 C206 S207 & $116.38(10)$ \\
\hline C101 C106 C105 & $121.26(13)$ & C201 C206 C205 & $121.05(13)$ \\
\hline C105 C106 S107 & $117.53(10)$ & C205 C206 S207 & $122.42(11)$ \\
\hline O109 C109 O110 & $122.75(12)$ & O209 C209 O210 & $123.02(12)$ \\
\hline O109 C109 N108 & $128.89(12)$ & O209 C209 N208 & $128.38(13)$ \\
\hline O110 C109 N108 & $108.36(11)$ & O210 C209 N208 & $108.60(11)$ \\
\hline O110 C111 C112 & $112.19(11)$ & O210 C211 C212 & $112.19(11)$ \\
\hline C113 C112 C111 & $120.07(12)$ & C213 C212 C211 & $120.22(12)$ \\
\hline C117 C112 C111 & $120.79(12)$ & C217 C212 C211 & $120.73(12)$ \\
\hline C117 C112 C113 & $119.12(13)$ & C217 C212 C213 & $119.00(13)$ \\
\hline C114 C113 C112 & $120.22(13)$ & C214 C213 C212 & $120.43(13)$ \\
\hline C113 C114 C115 & $120.45(14)$ & C215 C214 C213 & $119.89(13)$ \\
\hline C116 C115 C114 & $119.72(14)$ & C216 C215 C214 & $120.11(14)$ \\
\hline C115 C116 C117 & $120.01(14)$ & C215 C216 C217 & $119.79(14)$ \\
\hline C112 C117 C116 & $120.48(14)$ & C212 C217 C216 & $120.77(13)$ \\
\hline
\end{tabular}

Table 6 Torsion Angles for $\mathbf{3 j}$.

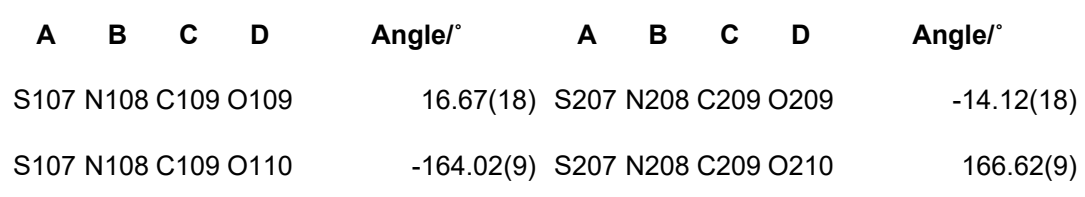




\begin{tabular}{|c|c|c|c|}
\hline F103 C103 C104 C105 & $179.79(12)$ & F203 C203 C204 C205 & $-179.39(12)$ \\
\hline O107 S107 N108 C109 & $-94.75(10)$ & O207 S207 N208 C209 & $88.55(10)$ \\
\hline O107 S107 C106 C101 & $-126.06(11)$ & O207 S207 C206 C201 & $-70.77(11)$ \\
\hline O107 S107 C106 C105 & $58.46(11)$ & O207 S207 C206 C205 & $113.57(12)$ \\
\hline O110 C111 C112 C113 & 44.55(16) & O210 C211 C212 C213 & $-51.14(17)$ \\
\hline O110 C111 C112 C117 & $-137.41(13)$ & O210 C211 C212 C217 & $131.60(13)$ \\
\hline N108 S107 O107 C107 & $-48.53(12)$ & N208 S207 O207 C207 & $51.92(12)$ \\
\hline N108 S107 C106 C101 & $-11.63(12)$ & N208 S207 C206 C201 & $174.89(11)$ \\
\hline N108 S107 C106 C105 & $172.89(11)$ & N208 S207 C206 C205 & $-0.77(13)$ \\
\hline C101 C102 C103 F103 & 179.83(12) & C201 C202 C203 F203 & $179.23(12)$ \\
\hline C101 C102 C103 C104 & $-0.9(2)$ & C201 C202 C203 C204 & $-0.4(2)$ \\
\hline C102 C101 C106 S107 & $-175.38(10)$ & C202 C201 C206 S207 & $-176.17(11)$ \\
\hline C102 C101 C106 C105 & $-0.1(2)$ & C202 C201 C206 C205 & $-0.4(2)$ \\
\hline C102 C103 C104 C105 & $0.5(2)$ & C202 C203 C204 C205 & $0.3(2)$ \\
\hline C103 C104 C105 C106 & $0.1(2)$ & C203 C204 C205 C206 & $-0.2(2)$ \\
\hline C104 C105 C106 S107 & $175.16(10)$ & C204 C205 C206 S207 & $175.74(10)$ \\
\hline C104 C105 C106 C101 & $-0.3(2)$ & C204 C205 C206 C201 & $0.3(2)$ \\
\hline C106 S107 O107 C107 & $56.70(11)$ & C206 S207 O207 C207 & $-53.98(12)$ \\
\hline C106 S107 N108 C109 & $158.82(9)$ & C206 S207 N208 C209 & $-164.07(9)$ \\
\hline C106 C101 C102 C103 & $0.6(2)$ & C206 C201 C202 C203 & $0.5(2)$ \\
\hline C109 O110 C111 C112 & $73.71(14)$ & C209 O210 C211 C212 & $-78.27(14)$ \\
\hline C111 O110 C109 O109 & $1.00(18)$ & C211 O210 C209 O209 & $-2.15(18)$ \\
\hline C111 O110 C109 N108 & $-178.36(10)$ & C211 O210 C209 N208 & $177.16(10)$ \\
\hline C111 C112 C113 C114 & 178.57(12) & C211 C212 C213 C214 & $-177.42(13)$ \\
\hline C111 C112 C117 C116 & $-178.06(12)$ & C211 C212 C217 C216 & 176.68(13) \\
\hline C112 C113 C114 C115 & $-0.4(2)$ & C212 C213 C214 C215 & $0.9(2)$ \\
\hline C113 C112 C117 C116 & $0.0(2)$ & C213 C212 C217 C216 & $-0.6(2)$ \\
\hline C113 C114 C115 C116 & $-0.2(2)$ & C213 C214 C215 C216 & $-1.0(2)$ \\
\hline C114 C115 C116 C117 & $0.7(2)$ & C214 C215 C216 C217 & $0.2(2)$ \\
\hline C115 C116 C117 C112 & $-0.6(2)$ & C215 C216 C217 C212 & $0.6(2)$ \\
\hline C117 C112 C113 C114 & $0.5(2)$ & C217 C212 C213 C214 & $-0.1(2)$ \\
\hline
\end{tabular}

Table 7 Hydrogen Atom Coordinates $\left(A \times 10^{4}\right)$ and Isotropic Displacement Parameters $\left(\AA^{2} \times 10^{3}\right)$ for $3 j$.

\begin{tabular}{|c|c|c|c|c|}
\hline Atom & $x$ & $y$ & $z$ & $\mathrm{U}(\mathrm{eq})$ \\
\hline H101 & 1741.82 & -2343.14 & 1555.31 & 34 \\
\hline
\end{tabular}




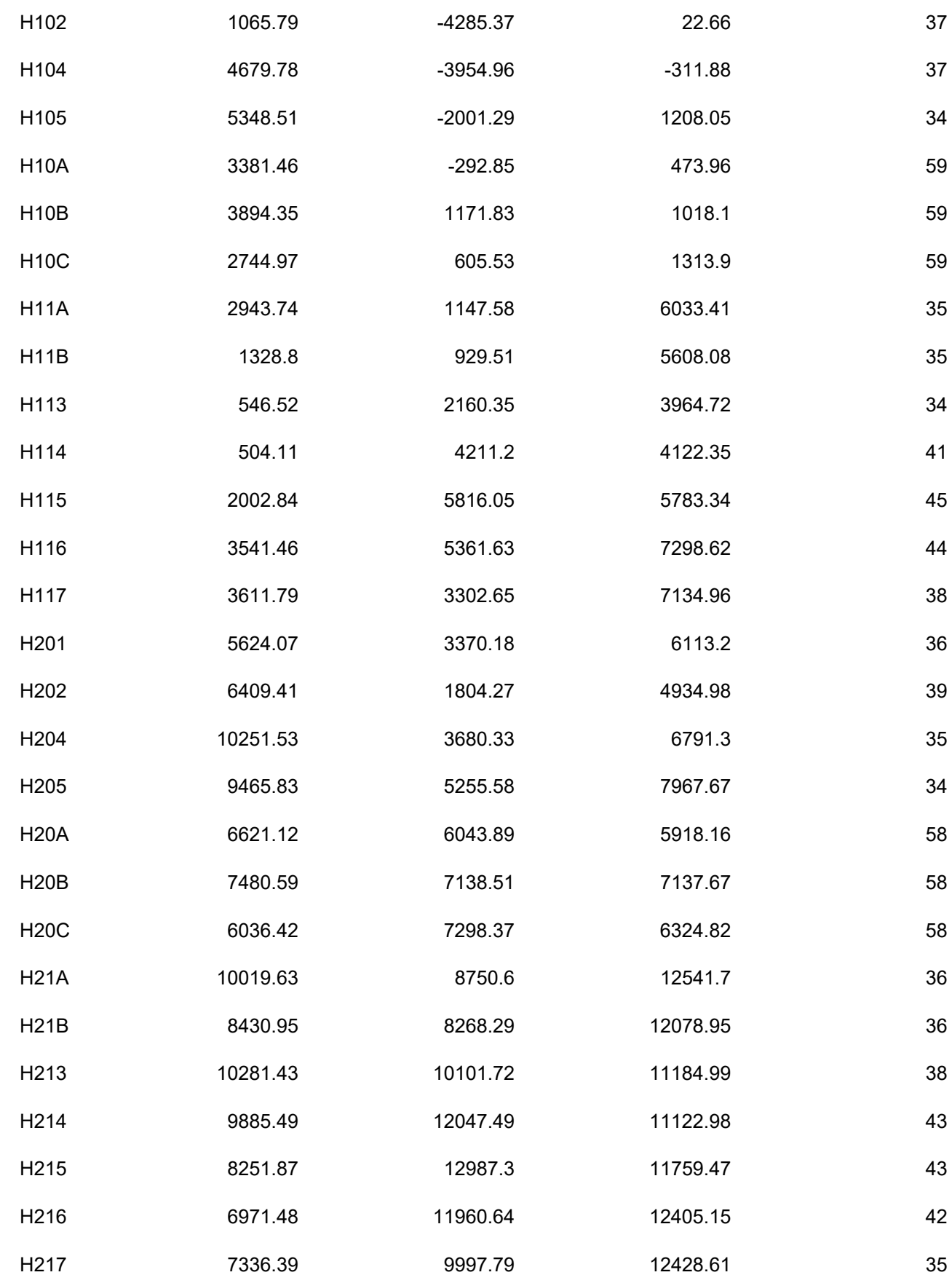

\section{Refinement model description}

Number of restraints - 0 , number of constraints - unknown.

Details:

1. Fixed Uiso

At 1.2 times of:

All $\mathrm{C}(\mathrm{H})$ groups, All $\mathrm{C}(\mathrm{H}, \mathrm{H})$ groups

At 1.5 times of:

All $\mathrm{C}(\mathrm{H}, \mathrm{H}, \mathrm{H})$ groups

2.a Secondary $\mathrm{CH} 2$ refined with riding coordinates:

C111(H11A,H11B), C211(H21A,H21B)

2.b Aromatic/amide $\mathrm{H}$ refined with riding coordinates:

C101(H101), C102(H102), C104(H104), C105(H105), C113(H113), C114(H114),

C115(H115), C116(H116), C117(H117), C201(H201), C202(H202), C204(H204),

C205(H205), C213(H213), C214(H214), C215(H215), C216(H216), C217(H217)

2.c Idealised $\mathrm{Me}$ refined as rotating group:

C107(H10A,H10B,H10C), C207(H2OA,H20B,H20C) 


\section{Benzyl-(phenyl(piperidin-1-yl)- $\lambda^{4}$-sulfanylidene)carbamate (4a)}

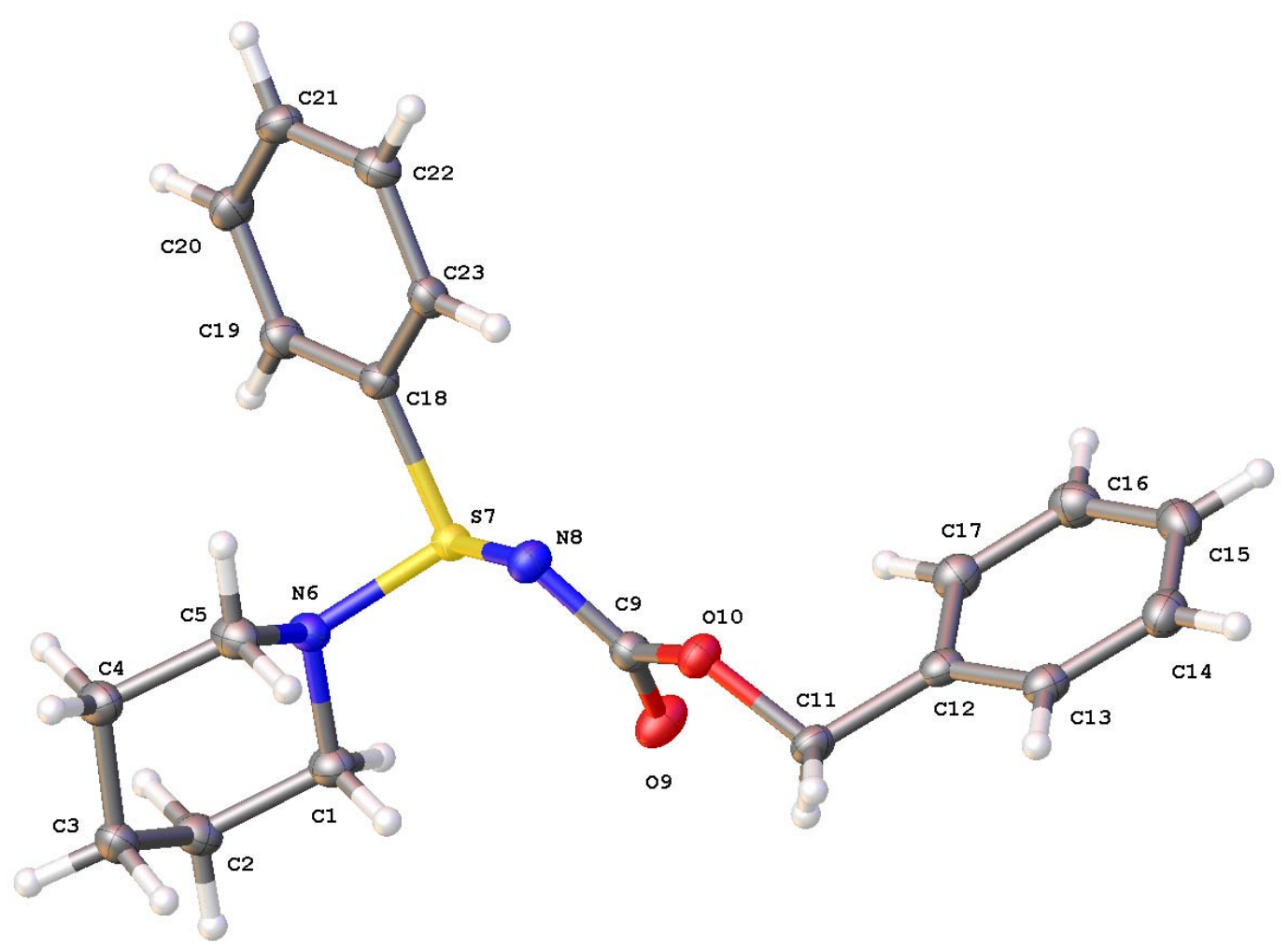

solid state structure of $\mathbf{4 a}$ with atom labelling and thermal ellipsoids drawn at 50\% probability level

\section{Crystal structure determination of $4 a$}

The asymmetric unit contains the compound, there are two molecules in the unit cell related by an inversion centre.

Nothing unusual to report in the structure or refinement

\section{Experimental}

Single crystals of $\mathrm{C}_{19} \mathrm{H}_{22} \mathrm{~N}_{2} \mathrm{O}_{2} \mathrm{~S} 4 \mathrm{a}$ were grown from $\mathrm{Et}_{2} \mathrm{O}$. A suitable crystal was selected and mounted on a glass fibre with Fomblin oil and placed on a Rigaku Oxford Diffraction Synergy-S diffractometer with a duel source equipped with a Hybrid pixel array detector. Using Olex2 [1], the structure was solved with the SheIXT [2] structure solution program using Intrinsic Phasing and refined with the ShelXL [3] refinement package using Least Squares minimisation.

1. Dolomanov, O.V., Bourhis, L.J., Gildea, R.J, Howard, J.A.K. \& Puschmann, H. (2009), J. Appl. Cryst. 42, 339-341.

2. Sheldrick, G.M. (2015). Acta Cryst. A71, 3-8.

3. Sheldrick, G.M. (2015). Acta Cryst. C71, 3-8.

Crystal Data for $\mathrm{C}_{19} \mathrm{H}_{22} \mathrm{~N}_{2} \mathrm{O}_{2} \mathrm{~S}(M=342.44 \mathrm{~g} / \mathrm{mol})$ : triclinic, space group P-1 (no. 2), $a=5.74360(10) \AA, b=11.2394(2) \AA, c=13.8843(2) \AA, \alpha=$ $99.1640(10)^{\circ}, \beta=91.0830(10)^{\circ}, \gamma=100.953(2)^{\circ}, V=867.62(3) \AA^{3}, Z=2, T=100(2) \mathrm{K}, \mu($ CuKa $)=1.763 \mathrm{~mm}^{-1}, D$ calc $=1.311 \mathrm{~g} / \mathrm{cm}^{3}, 34095$ reflections measured $\left(6.456^{\circ} \leq 2 \Theta \leq 159.898^{\circ}\right), 3700$ unique $\left(R_{\text {int }}=0.0547, R_{\text {sigma }}=0.0217\right)$ which were used in all calculations. The final $R_{1}$ was $0.0351(\mathrm{I}>2 \sigma(\mathrm{I}))$ and $w R_{2}$ was 0.0993 (all data). 
Table 1 Crystal data and structure refinement for $\mathbf{4 a}$.

\begin{tabular}{|c|c|}
\hline Identification code & $\mathrm{rl} 6$ \\
\hline Empirical formula & $\mathrm{C}_{19} \mathrm{H}_{22} \mathrm{~N}_{2} \mathrm{O}_{2} \mathrm{~S}$ \\
\hline Formula weight & 342.44 \\
\hline Temperature/K & $100(2)$ \\
\hline Crystal system & triclinic \\
\hline Space group & $\mathrm{P}-1$ \\
\hline $\mathrm{a} / \AA \AA$ & $5.74360(10)$ \\
\hline $\mathrm{b} / \AA \AA$ & $11.2394(2)$ \\
\hline$c / \AA ̊$ & $13.8843(2)$ \\
\hline$\alpha /{ }^{\circ}$ & $99.1640(10)$ \\
\hline$\beta /^{\circ}$ & $91.0830(10)$ \\
\hline$\gamma^{\circ}{ }^{\circ}$ & $100.953(2)$ \\
\hline Volume $/ \AA^{3}$ & $867.62(3)$ \\
\hline Z & 2 \\
\hline$\rho_{\text {calc }} g / \mathrm{cm}^{3}$ & 1.311 \\
\hline$\mu / \mathrm{mm}^{-1}$ & 1.763 \\
\hline$F(000)$ & 364.0 \\
\hline Crystal size $/ \mathrm{mm}^{3}$ & $0.2 \times 0.06 \times 0.04$ \\
\hline Radiation & $\operatorname{CuKa}(\lambda=1.54184)$ \\
\hline \multicolumn{2}{|c|}{$2 \Theta$ range for data collection $/{ }^{\circ} 6.456$ to 159.898} \\
\hline Index ranges & $-7 \leq h \leq 7,-14 \leq k \leq 14,-17 \leq \mathrm{I} \leq 17$ \\
\hline Reflections collected & 34095 \\
\hline Independent reflections & $3700\left[R_{\text {int }}=0.0547, R_{\text {sigma }}=0.0217\right]$ \\
\hline Data/restraints/parameters & $3700 / 0 / 217$ \\
\hline Goodness-of-fit on $F^{2}$ & 1.150 \\
\hline Final $R$ indexes $[l>=2 \sigma(I)]$ & $\mathrm{R}_{1}=0.0351, \mathrm{wR}_{2}=0.0987$ \\
\hline Final $\mathrm{R}$ indexes [all data] & $\mathrm{R}_{1}=0.0362, \mathrm{wR}_{2}=0.0993$ \\
\hline Largest diff. peak/hole / e $\AA^{-3}$ & $0.30 /-0.41$ \\
\hline
\end{tabular}

Table 2 Fractional Atomic Coordinates $\left(\times 10^{4}\right)$ and Equivalent Isotropic Displacement Parameters $\left(A^{2} \times 10^{3}\right)$ for $4 a$. $U_{\text {eq }}$ is defined as $1 / 3$ of of the trace of the orthogonalised $U_{I J}$ tensor.

Atom

S7

010 $x$

$932.7(5)$

$5691.1(16)$ $y$

5594.6(3)

3977.2(8) $\boldsymbol{z}$

$\mathrm{U}(\mathrm{eq})$

7279.6(2)

7779.8(7)
17.30(11)

20.6(2) 


\begin{tabular}{|c|c|c|c|c|}
\hline O9 & $1980.1(17)$ & $3839.2(9)$ & $8335.5(8)$ & $27.4(2)$ \\
\hline N8 & $3527.7(19)$ & $5225.9(10)$ & $7308.1(8)$ & $20.4(2)$ \\
\hline N6 & $858.9(19)$ & $6870.0(10)$ & $8089.0(8)$ & $19.4(2)$ \\
\hline C18 & $1082(2)$ & $6255.3(11)$ & $6190.7(9)$ & $18.2(3)$ \\
\hline $\mathrm{C} 12$ & $5786(2)$ & $1804.9(12)$ & $7596.5(9)$ & $19.5(3)$ \\
\hline $\mathrm{C} 23$ & $2762(2)$ & $6027.4(11)$ & $5514.1(9)$ & $19.5(3)$ \\
\hline $\mathrm{Cg}$ & $3535(2)$ & $4314.8(11)$ & $7848.7(9)$ & $19.0(3)$ \\
\hline C22 & $2703(2)$ & $6471.8(12)$ & $4635.0(10)$ & $21.9(3)$ \\
\hline C19 & $-674(2)$ & $6902.9(12)$ & $6004.5(10)$ & $21.7(3)$ \\
\hline C21 & $984(2)$ & $7136.4(12)$ & $4446.9(10)$ & $22.8(3)$ \\
\hline $\mathrm{C} 13$ & $7514(2)$ & $1084.6(12)$ & $7584.9(10)$ & $21.8(3)$ \\
\hline C11 & $6080(2)$ & $3007.0(12)$ & $8292.7(9)$ & $20.5(3)$ \\
\hline C1 & $40(2)$ & $6561.5(12)$ & $9042.9(10)$ & $22.4(3)$ \\
\hline $\mathrm{C} 20$ & $-694(2)$ & $7349.8(13)$ & $5128.4(10)$ & $23.5(3)$ \\
\hline C3 & $1804(3)$ & $8733.4(12)$ & $9810.5(10)$ & $24.2(3)$ \\
\hline C14 & $7260(3)$ & $-22.1(13)$ & $6942.0(11)$ & $25.8(3)$ \\
\hline C2 & $-413(2)$ & $7717.2(13)$ & $9686.3(10)$ & $24.3(3)$ \\
\hline C17 & $3801(2)$ & $1401.2(13)$ & $6946.0(10)$ & $23.4(3)$ \\
\hline C5 & $3050(2)$ & $7822.1(12)$ & $8184.4(10)$ & $23.8(3)$ \\
\hline C15 & $5290(3)$ & $-409.0(13)$ & $6293.8(10)$ & $26.6(3)$ \\
\hline C16 & $3568(3)$ & $306.0(13)$ & $6295.7(10)$ & $26.6(3)$ \\
\hline C4 & 2622(3) & $8993.0(13)$ & $8813.5(10)$ & $26.5(3)$ \\
\hline
\end{tabular}

Table 3 Anisotropic Displacement Parameters $\left(\AA^{2} \times 10^{3}\right)$ for $4 a$. The Anisotropic displacement factor exponent takes the form: $2 \pi^{2}\left[h^{2} a^{* 2} U_{11}+2 h k a^{*} b^{*} U_{12}+\ldots\right]$.

\begin{tabular}{|c|c|c|c|c|c|c|}
\hline Atom & $U_{11}$ & $\mathbf{U}_{22}$ & $\mathbf{U}_{33}$ & $\mathbf{U}_{23}$ & $\mathbf{U}_{13}$ & $U_{12}$ \\
\hline S7 & $18.29(17)$ & $17.96(16)$ & $15.84(17)$ & $2.93(11)$ & $0.46(11)$ & $3.92(11)$ \\
\hline 010 & $19.6(4)$ & $21.8(5)$ & $23.0(5)$ & $7.9(4)$ & $2.2(4)$ & $6.9(4)$ \\
\hline O9 & $23.4(5)$ & $31.8(5)$ & $33.0(5)$ & $16.7(4)$ & $8.4(4)$ & $10.3(4)$ \\
\hline N8 & $19.6(5)$ & $22.0(5)$ & $21.0(5)$ & $6.3(4)$ & $0.8(4)$ & $5.4(4)$ \\
\hline N6 & $21.6(5)$ & $19.6(5)$ & $16.3(5)$ & $2.4(4)$ & $1.4(4)$ & $2.6(4)$ \\
\hline C18 & $20.9(6)$ & $17.0(6)$ & $15.8(6)$ & $2.1(4)$ & $-0.9(5)$ & $2.4(5)$ \\
\hline $\mathrm{C} 12$ & $20.5(6)$ & $22.6(6)$ & $17.2(6)$ & $7.5(5)$ & $3.1(5)$ & $4.8(5)$ \\
\hline C23 & $19.9(6)$ & $19.3(6)$ & 19.1(6) & $0.9(5)$ & $-0.7(5)$ & $5.0(5)$ \\
\hline C9 & 19.1(6) & $20.1(6)$ & $17.5(6)$ & $1.4(5)$ & $0.3(5)$ & $4.8(5)$ \\
\hline C22 & $22.5(6)$ & $23.7(6)$ & $18.2(6)$ & $1.4(5)$ & $1.8(5)$ & $3.0(5)$ \\
\hline
\end{tabular}




\begin{tabular}{|c|c|c|c|c|c|c|}
\hline C19 & $22.0(6)$ & $23.1(6)$ & $20.4(6)$ & $2.2(5)$ & $1.1(5)$ & $6.8(5)$ \\
\hline C21 & $27.0(7)$ & $23.5(6)$ & $18.2(6)$ & $5.7(5)$ & $-2.1(5)$ & $3.6(5)$ \\
\hline C13 & $22.2(6)$ & $25.3(6)$ & $19.7(6)$ & $7.4(5)$ & $2.1(5)$ & $6.0(5)$ \\
\hline C11 & $22.2(6)$ & $22.9(6)$ & $18.6(6)$ & $6.5(5)$ & $-0.8(5)$ & 7.4(5) \\
\hline C1 & $25.0(7)$ & $23.3(6)$ & $18.9(6)$ & $4.7(5)$ & $2.9(5)$ & $3.3(5)$ \\
\hline C20 & $24.7(7)$ & 24.1(6) & $23.4(7)$ & $5.1(5)$ & $-2.6(5)$ & $8.4(5)$ \\
\hline C3 & $30.2(7)$ & $22.5(6)$ & $19.0(6)$ & $-0.1(5)$ & $-3.0(5)$ & $6.1(5)$ \\
\hline C14 & $28.6(7)$ & $25.2(7)$ & $27.1(7)$ & $8.4(6)$ & $6.4(6)$ & $9.7(6)$ \\
\hline C2 & $25.9(7)$ & $28.1(7)$ & $18.9(6)$ & $1.5(5)$ & $2.5(5)$ & $6.9(5)$ \\
\hline C17 & 20.6(6) & $25.6(7)$ & $24.7(7)$ & $6.6(5)$ & $0.9(5)$ & 4.6(5) \\
\hline C5 & $24.5(7)$ & 23.6(7) & $20.5(6)$ & $2.4(5)$ & $1.9(5)$ & $-1.5(5)$ \\
\hline C15 & $33.1(8)$ & $21.3(7)$ & $24.0(7)$ & $2.2(5)$ & $5.0(6)$ & $2.7(6)$ \\
\hline C16 & $26.4(7)$ & $27.9(7)$ & $23.9(7)$ & $4.4(6)$ & $-1.5(5)$ & $1.2(5)$ \\
\hline C4 & $34.2(7)$ & $20.9(6)$ & $22.1(7)$ & $2.9(5)$ & $-1.5(6)$ & $0.2(5)$ \\
\hline
\end{tabular}

Table 4 Bond Lengths for $4 a$.

\begin{tabular}{|c|c|c|c|c|c|}
\hline Atom & Atom & Length/Å & Atom & Atom & Length/Å \\
\hline S7 & N8 & $1.6225(11)$ & C12 & C17 & $1.3983(18)$ \\
\hline S7 & N6 & $1.6826(11)$ & $\mathrm{C} 23$ & C22 & $1.3926(19)$ \\
\hline S7 & C18 & $1.7840(13)$ & $\mathrm{C} 22$ & C21 & $1.3902(19)$ \\
\hline 010 & C9 & $1.3632(15)$ & C19 & $\mathrm{C} 20$ & $1.3886(19)$ \\
\hline 010 & C11 & $1.4423(15)$ & C21 & $\mathrm{C} 20$ & $1.390(2)$ \\
\hline 09 & $\mathrm{C9}$ & $1.2234(17)$ & $\mathrm{C} 13$ & C14 & $1.392(2)$ \\
\hline N8 & C9 & $1.3632(17)$ & $\mathrm{C} 1$ & C2 & $1.5233(18)$ \\
\hline N6 & C1 & $1.4844(17)$ & C3 & C2 & $1.527(2)$ \\
\hline N6 & C5 & $1.4776(17)$ & $\mathrm{C} 3$ & C4 & $1.524(2)$ \\
\hline C18 & $\mathrm{C} 23$ & $1.3895(18)$ & C14 & C15 & $1.389(2)$ \\
\hline C18 & C19 & $1.3940(18)$ & C17 & C16 & $1.388(2)$ \\
\hline C12 & C13 & $1.3939(18)$ & C5 & C4 & $1.5232(19)$ \\
\hline C12 & C11 & $1.5098(18)$ & C15 & C16 & $1.388(2)$ \\
\hline
\end{tabular}




\begin{tabular}{|c|c|c|c|c|c|c|c|}
\hline \multicolumn{3}{|c|}{ Atom Atom Atom } & \multirow{2}{*}{$\begin{array}{l}\text { Angle }^{\circ} \\
\qquad 111.23(6)\end{array}$} & \multicolumn{3}{|c|}{ Atom Atom Atom } & \multirow{2}{*}{$\begin{array}{l}\text { Angle }^{\circ} \\
\qquad 129.66(12)\end{array}$} \\
\hline N8 & S7 & N6 & & O9 & C9 & N8 & \\
\hline N8 & S7 & C18 & $100.05(6)$ & $\mathrm{C} 21$ & $\mathrm{C} 22$ & $\mathrm{C} 23$ & $119.96(12)$ \\
\hline N6 & S7 & C18 & $98.55(6)$ & $\mathrm{C} 20$ & C19 & C18 & 118.63(13) \\
\hline C9 & 010 & C11 & $117.42(10)$ & $\mathrm{C} 20$ & C21 & $\mathrm{C} 22$ & $120.31(12)$ \\
\hline C9 & N8 & S7 & $110.80(9)$ & C14 & C13 & C12 & $120.58(13)$ \\
\hline $\mathrm{C} 1$ & N6 & S7 & $111.30(8)$ & 010 & C11 & $\mathrm{C} 12$ & $110.46(10)$ \\
\hline C5 & N6 & S7 & $114.90(9)$ & N6 & C1 & $\mathrm{C} 2$ & $109.20(11)$ \\
\hline C5 & N6 & $\mathrm{C} 1$ & $113.02(10)$ & $\mathrm{C} 19$ & $\mathrm{C} 20$ & $\mathrm{C} 21$ & $120.46(12)$ \\
\hline $\mathrm{C} 23$ & C18 & S7 & $120.53(10)$ & C4 & C3 & $\mathrm{C} 2$ & $109.99(11)$ \\
\hline $\mathrm{C} 23$ & C18 & C19 & $121.57(12)$ & C15 & C14 & $\mathrm{C} 13$ & $120.04(13)$ \\
\hline C19 & $\mathrm{C} 18$ & S7 & $117.62(10)$ & $\mathrm{C} 1$ & $\mathrm{C} 2$ & C3 & $110.89(11)$ \\
\hline $\mathrm{C} 13$ & $\mathrm{C} 12$ & C11 & $120.26(11)$ & $\mathrm{C} 16$ & $\mathrm{C} 17$ & $\mathrm{C} 12$ & $120.46(13)$ \\
\hline C13 & C12 & C17 & $118.86(12)$ & N6 & C5 & $\mathrm{C} 4$ & $109.56(11)$ \\
\hline C17 & C12 & C11 & $120.88(11)$ & $\mathrm{C} 16$ & C15 & $\mathrm{C} 14$ & $119.77(13)$ \\
\hline C18 & $\mathrm{C} 23$ & $\mathrm{C} 22$ & $119.04(12)$ & C15 & C16 & $\mathrm{C} 17$ & $120.27(13)$ \\
\hline 010 & $\mathrm{C9}$ & N8 & $108.04(11)$ & C5 & C4 & C3 & $110.39(11)$ \\
\hline O9 & C9 & O10 & $122.30(11)$ & & & & \\
\hline
\end{tabular}

Table 6 Hydrogen Atom Coordinates $\left(\AA \times 10^{4}\right)$ and Isotropic Displacement Parameters $\left(\AA^{2} \times 10^{3}\right)$ for 4 a.

\begin{tabular}{lrrrr} 
Atom & \multicolumn{3}{c}{$\boldsymbol{y}$} & $\mathrm{U}(\mathbf{e q})$ \\
H23 & 3933.75 & 5575.04 & 5649.24 & 23 \\
H22 & 3835.98 & 6320.89 & 4164.17 & 26 \\
H19 & -1834.22 & 7035.7 & 6467.43 & 26 \\
H21 & 957.21 & 7446.22 & 3849.69 & 27 \\
H13 & 8877.28 & 1351.28 & 8019.6 & 26 \\
H11A & 4932.15 & 2923.45 & 8812.37 & 25 \\
H11B & 7701.53 & 3216.35 & 8605.47 & 25 \\
H1A & 1263.21 & 6226.29 & 9366.61 & 27 \\
H1B & -1437.86 & 5928.95 & 8939.85 & 27 \\
H20 & -1862.47 & 7804.47 & 4993.91 & 28 \\
H3A & 1448.84 & 9489.86 & 10199.49 & 29 \\
H3B & 3086.56 & 8478.12 & 10166.15 & 29 \\
H14 & 8435.21 & -513.13 & 6946.6 & 31 \\
H2A & -883.03 & 7524.32 & 10334.94 & 29
\end{tabular}




$\begin{array}{lrrrr}\text { H2B } & -1736.5 & 8005.84 & 9387.31 & 29 \\ \mathrm{H} 17 & 2603.15 & 1879.84 & 6949.13 & 28 \\ \mathrm{H} 5 \mathrm{~A} & 3517.58 & 7993.13 & 7529.91 & 29 \\ \mathrm{H} 5 \mathrm{~B} & 4356.95 & 7525.88 & 8488.25 & 29 \\ \mathrm{H} 15 & 5121.9 & -1160.5 & 5850.72 & 32 \\ \mathrm{H} 16 & 2224.1 & 45.02 & 5850.16 & 32 \\ \mathrm{H} 4 \mathrm{~A} & 1398.32 & 9321.15 & 8483.69 & 32 \\ \mathrm{H} 4 \mathrm{~B} & 4106.89 & 9620.99 & 8897.85 & 32\end{array}$

\section{Refinement model description}

Number of restraints - 0 , number of constraints - unknown.

Details:

1. Fixed Uiso

At 1.2 times of:

All $\mathrm{C}(\mathrm{H})$ groups, All $\mathrm{C}(\mathrm{H}, \mathrm{H})$ groups

2.a Secondary $\mathrm{CH} 2$ refined with riding coordinates:

C11(H11A,H11B), C1(H1A,H1B), C3(H3A,H3B), C2(H2A,H2B), C5(H5A,H5B), C4(H4A,H4B)

2.b Aromatic/amide $\mathrm{H}$ refined with riding coordinates:

C23(H23), C22(H22), C19(H19), C21(H21), C13(H13), C20(H20), C14(H14),

$\mathrm{C} 17(\mathrm{H} 17), \mathrm{C} 15(\mathrm{H} 15), \mathrm{C} 16(\mathrm{H} 16)$

This report has been created with Olex2, compiled on 2018.05.29 svn.r3510 for OlexSys. Please let us know if there are any errors or if you would like to have additional features. 


\section{REFERENCES}

(1) a) Briggs, E. L.; Tota, A.; Colella, M.; Degennaro, L.; Luisi, R.; Bull, J. A. Synthesis of Sulfonimidamides from Sulfenamides via an Alkoxy-amino- $\lambda 6$-sulfanenitrile Intermediate. Angewandte Chemie International Edition 2019, 58 (40), 14303-14310. b) Yakan, H.; Kutuk, H. Comparison of conventional and microwave-assisted synthesis of some new sulfenamides under free catalyst and ligand. Monatsh Chem 2018, 149, 2047-2057, https://doi.org/10.1007/s00706-018-2261-4.

(2)Taniguchi, N. Convenient Synthesis of Unsymmetrical Organochalcogenides Using Organoboronic Acids with Dichalcogenides via Cleavage of the S-S, Se-Se, or Te-Te Bond by a Copper Catalyst J. Org. Chem. 2007, 72, 1241-1245.

(3) Taniguchi, N. Unsymmetrical Disulfide and Sulfenamide Synthesis via Reactions of Thiosulfonates with Thiols or Amines. Tetrahedron 2017, 73 (15), 2030-2035

(4) Qin, L.; Zhou, Z.; Wei, J.; Yan, T.; Wen, H. General and Efficient Synthesis of O-Sulfonylhydroxylamine Derivatives. Synthetic Communications 2010, 40 (5), 642-646.

(5) For the synthesis of $\mathrm{N}$-carboisobutyloxyhydroxylamine: a) Lu, D.-F.; Zhu, C.-L.; Jia, Z.-X.; Xu, H. Iron(II)Catalyzed Intermolecular Amino-Oxygenation of Olefins through the $\mathrm{N}-\mathrm{O}$ Bond Cleavage of Functionalized Hydroxylamines. Journal of the American Chemical Society 2014, 136 (38), 13186-13189; For the synthesis of isobutyl((methylsulfonyl)oxy)carbamate: b) Masruri; Willis, A. C.; McLeod, M. D. Osmium-Catalyzed Vicinal Oxyamination of Alkenes by N-(4-Toluenesulfonyloxy)carbamates. The Journal of Organic Chemistry 2012, 77 (19), 84808491. 